SANDIA REPORT

SAND90-0543 • UC-721

Unlimited Release

Printed July 1997

RECEIVED

AUG 011997

OSTI

\title{
SANTOS-A Two-Dimensional Finite Element Program for the Quasistatic, Large Deformation, Inelastic Response of Solids
}

Charles M. Stone

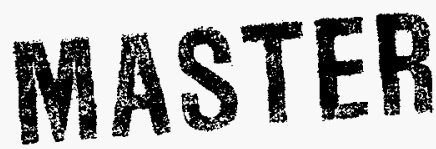

Prepared by

Sandia National Laboratories

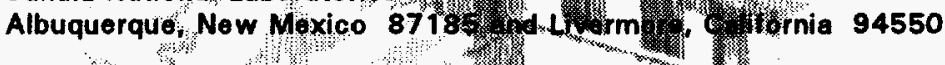

Sandia is a multiprogram laborator. perated by Sandia

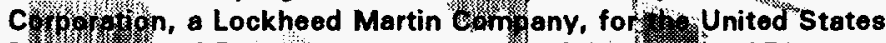

Doferthent of Energy under Contrad DE-ACO AL85000.

Approved for public release; distributiony unlimit

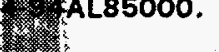


Issued by Sandia National Laboratories, operated for the United States Department of Energy by Sandia Corporation.

NOTICE: This report was prepared as an account of work sponsored by an agency of the United States Government. Neither the United States Government nor any agency thereof, nor any of their employees, nor any of their contractors, subcontractors, or their employees, makes any warranty, express or implied, or assumes any legal liability or responsibility for the accuracy, completeness, or usefulness of any information, apparatus, product, or process disclosed, or represents that its use would not infringe privately owned rights. Reference herein to any specific commercial product, process, or service by trade name, trademark, manufacturer, or otherwise, does not necessarily constitute or imply its endorsement, recommendation, or favoring by the United States Government, any agency thereof, or any of their contractors or subcontractors. The views and opinions expressed herein do not necessarily state or reflect those of the United States Government, any agency thereof, or any of their contractors.

Printed in the United States of America. This report has been reproduced directly from the best available copy.

Available to DOE and DOE contractors from Office of Scientific and Technical Information

P.O. Box 62

Oak Ridge, TN 37831

Prices available from (615) 576-8401, FTS 626-8401

Available to the public from

National Technical Information Service

U.S. Department of Commerce

5285 Port Royal Rd

Springfield, VA 22161

NTIS price codes

Printed copy: A08

Microfiche copy: A01 


\title{
SANTOS-A Two-Dimensional Finite Element Program for the Quasistatic, Large Deformation, Inelastic Response of Solids
}

\author{
Charles M. Stone \\ Engineering and Manufacturing Mechanics Department \\ Sandia National Laboratories \\ P.O. Box 5800 \\ Albuquerque, NM 87185-0443
}

\begin{abstract}
SANTOS is a finite element program designed to compute the quasistatic, large deformation, inelastic response of two-dimensional planar or axisymmetric solids. The code is derived from the transient dynamic code PRONTO 2D. The solution strategy used to compute the equilibrium states is based on a self-adaptive dynamic relaxation solution scheme, which is based on explicit central difference pseudo-time integration and artificial mass proportional damping. The element used in SANTOS is a uniform strain 4-node quadrilateral element with an hourglass control scheme to control the spurious deformation modes. Finite strain constitutive models for many common engineering materials are included. A robust master-slave contact algorithm for modeling sliding contact is implemented. An interface for coupling to an external code is also provided.
\end{abstract}




\section{ACKNOWLEDGMENTS}

The author acknowledges the technical contributions of L.M. Taylor and D.P. Flanagan, who developed the PRONTO architecture and provided the framework from which SANTOS is derived. Much of this manual is derived from their original PRONTO manual. Significant contributions to the development of SANTOS were made by several of the early users. J.G. Argüello and G.W. Wellman ran the various versions of the code and provided constructive feedback about its performance and capabilities. Greg Sjaardema produced the early scripts and system procedures that provided the users with an easy way to run the code. Martin Heinstein took a new look at the contact surface problem and significantly improved both the location and application phases of the contact surface algorithm. H.S. Morgan's early work in integration of unified-creep-plasticity models provided the basis for integrating the time-dependent constitutive models. The efforts of the many other individuals who ran early versions of the code and provided helpful comments are gratefully acknowledged.

This report was prepared with the support of the Waste Isolation Pillot Plant (WIPP) Project. The support of WIPP Principal Investigator B.M. Butcher is acknowledged. 


\section{CONTENTS}

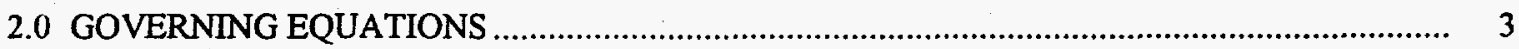

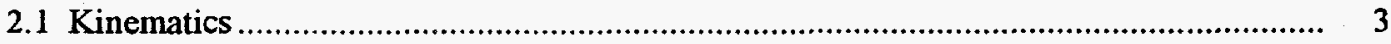

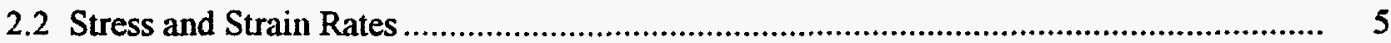

2.3 Fundamental Equations ............................................................................................... 8

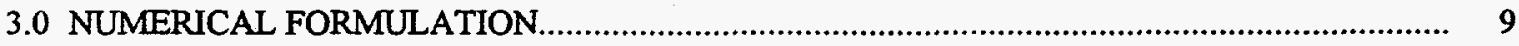

3.1 Four-Node Uniform Strain Element........................................................................... 9

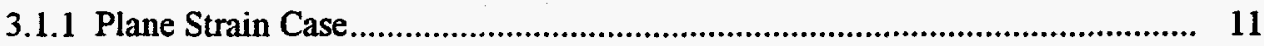

3.1.2 Axisymmetric Case ............................................................................... 14

3.1.3 Lumped Mass Matrix ......................................................................... 17

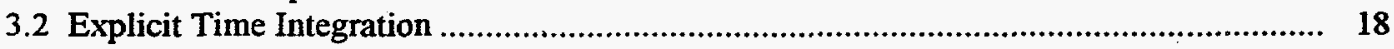

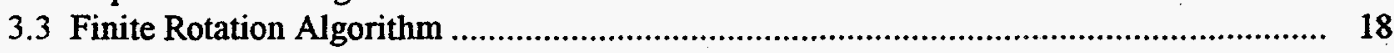

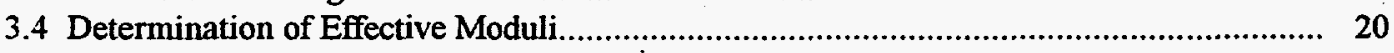

3.5 Determination of the Stable Time Increment ....................................................... 21

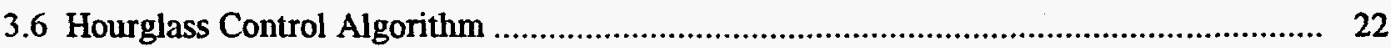

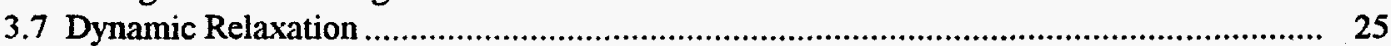

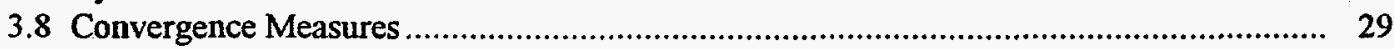

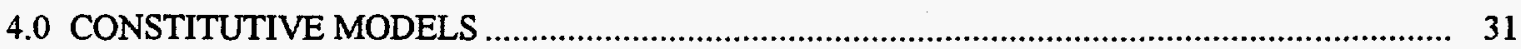

4.1 Integration of the Rate Equations.............................................................................. 31

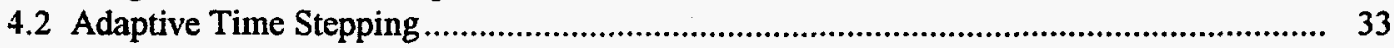

4.3 Basic Definitions and Assumptions................................................................... 34

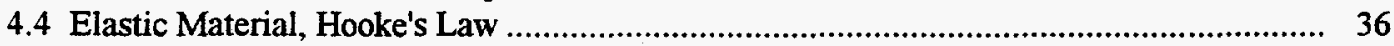

4.5 Elastic Plastic Material with Combined Kinematic and Isotropic Hardening................... 36

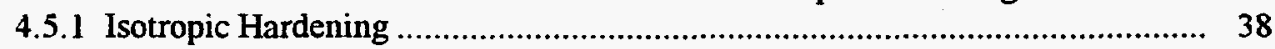

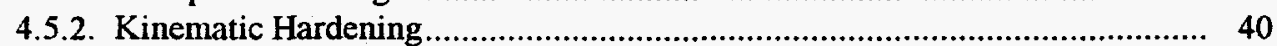

4.5.3 Combined Isotropic and Kinematic Hardening........................................... 42

4.5.4 Numerical Implementation................................................................. 44

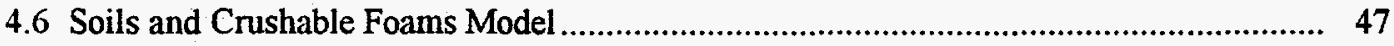

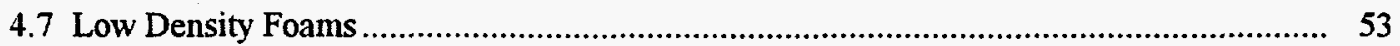

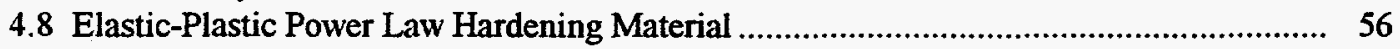

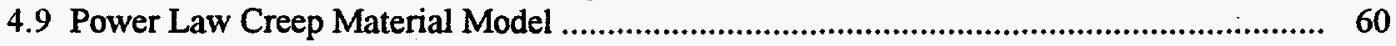

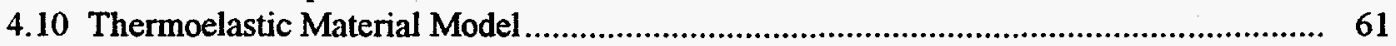

4.11 Thermoelastic-Plastic Power Law Hardening Material Model ................................... 63

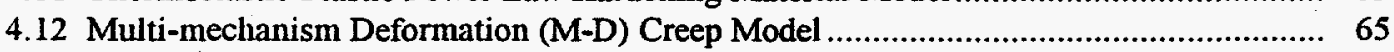

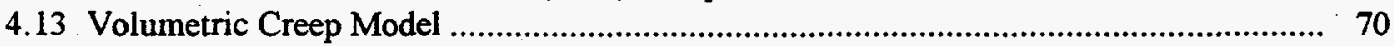

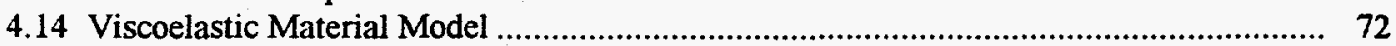

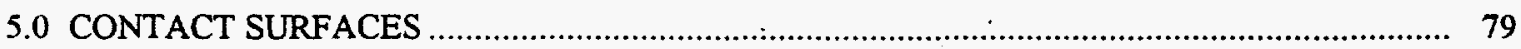

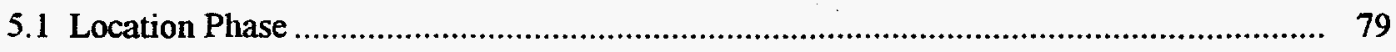

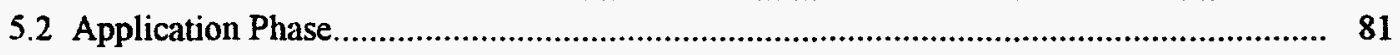

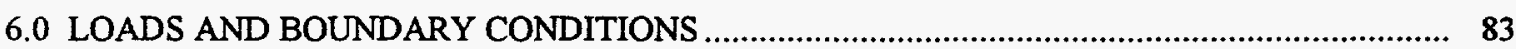

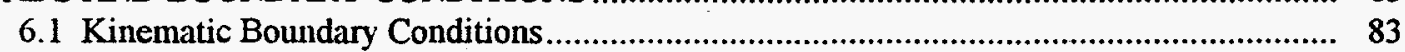

6.1.1 No Displacement Boundary Conditions.................................................... 83

6.1.2 Prescribed Displacement Boundary Conditions ............................................... 83 


\section{CONTENTS (Continued)}

6.1.3 Sloping Roller Boundary Conditions.................................................... 83

6.2 Traction Boundary Conditions and Distributed Loads ..................................................... 84

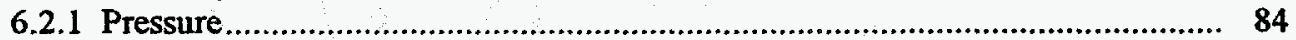

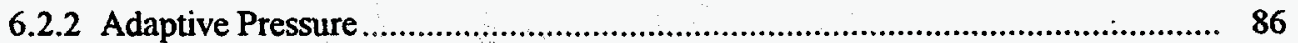

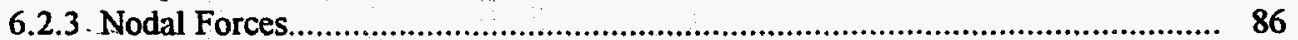

6.2.4 Gravity Forces, Body Forces, and Distributed Loads .................................... 86

6.2.5 Thermal Forces.............................................................................................. 87

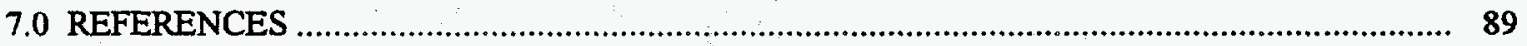

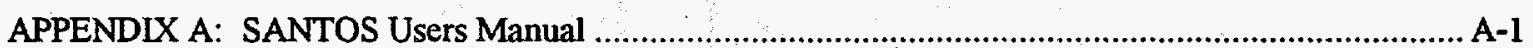

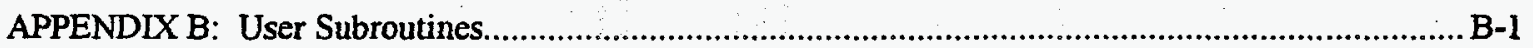

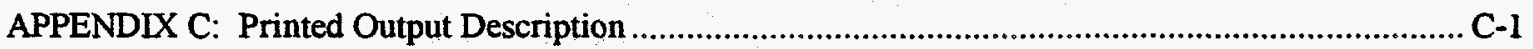

APPENDIX D: Adding a New Constitutive Model to SANTOS ..................................................... D-1

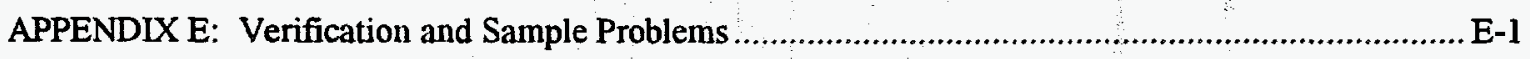




\section{DISCLAMMIER}

Portions of this document may be illegible in electronic image produets. Images are produced from the best available original document. 



\section{Figures}

2.1.1 Original, deformed, and intermediate configurations of a body

2.2.1 Computed stress-strain curves for a body undergoing simple shear using the Jaumann rate

2.2.2 Computed stress-strain curves for a body undergoing simple shear using the Green-

Naghdi rate

Mode shapes for the four-node constant strain quadrilateral element

A model equilibrium iteration sequence in a multi-dimensional configuration space of nodal point positions developed with dynamic relaxation showing convergence at load step $\mathbf{n}+1$

Conversion of data from a uniaxial tension test to equivalent plastic strain versus von Mises stress.

Geometric interpretation of the consistency condition for kinematic hardening Geometric interpretation of the incremental form of the consistency condition for combined hardening

Geometric interpretation of the radial return correction.

4.6.3 Pressure versus volumetric strain curve in terms of a user-defined curve, $F\left(\varepsilon_{\mathrm{v}}\right)$, for the soils and crushable foams material model

Possible loading cases for the pressure versus volumetric strain response using the soils and crushable foams material mode.

Schematic showing the effect of changing the master-slave designation between two surfaces. 
Intentionally Left Blank 


\subsection{INTRODUCTION}

SANTOS is a finite element program developed for quasistatic, large deformation, inelastic analysis of twodimensional solids. It is a powerful analysis tool that allows the user to address the solution of complex problems that include both material and geometric nonlinearities. The wide variety of constitutive models in the code allows SANTOS to be used for a wide class of problems from geomechanics to metal forming.

In 1986, Taylor and Flanagan at Sandia National Laboratories/New Mexico developed a new transient dynamics finite element code, which they named PRONTO (Taylor and Flanagan, 1987), that replaced the widely used HONDO II (Key et al., 1978) code. PRONTO employed the same explicit central difference time integration operator as HONDO II in addition to some new state-of-the-art features such as a uniform strain quadrilateral element with single point integration, improved critical time step estimates, and more robust contact surfaces. The code was written in a modular fashion with an easy-to-use interface for adding new constitutive models. The code architecture and storage schemes in PRONTO were also developed to take advantage of vector processing on the CRAY computer and to allow for the solution of extremely large problems. It seemed only natural, therefore, to take advantage of the development work of Taylor and Flanagan and adapt PRONTO for the solution of quasistatic problems by adding a self-adaptive dynamic relaxation scheme. A similar procedure was employed when adapting HONDO II to produce the SANCHO (Stone et al., 1985) quasistatic finite element code. The development and use of SANCHO showed that the same excellent results obtained for highly nonlinear transient dynamics problems using explicit methods could be achieved for quasistatic problems using an explicit method such as dynamic relaxation.

SANTOS belongs to a small but growing class of special purpose finite element codes which use iterative or indirect solution methods to achieve quasistatic solutions. A companion code to SANTOS is JAC (Biffle and Blanford, 1994) which utilizes a nonlinear conjugate gradient iterative scheme for obtaining quasistatic solutions. The solution algorithm in SANTOS is based on a self-adaptive dynamic relaxation scheme with uniform mesh homogenization which is identical to the method used in SANCHO. Because SANTOS is explicit in nature, there is no stiffness matrix to form or to factorize which reduces the amount of computer storage necessary for execution. Dynamic relaxation is not a new quasistatic solution technique with some of the early introductory papers on dynamic relaxation appearing in the mid-1960s. Dynamic relaxation is attractive for three reasons: 1) it is vectorizable, 2) it is versatile, and 3) it is reliable. Because it can be made explicit, it is highly vectorizable for modern digital calculations. In an explicit form, it is ideal for dealing with large deformations, finite strains, inelastic material behavior and contact surfaces. It is reliable in that if the algorithm converges and equilibrium is achieved, then the solution obtained will be good. An early introduction of the idea is given by Otter et al. (1966), but a more recent work which summarizes all of the significant contributions on the topic since Otter et al. can be found in Underwood (1983). Additional information on dynamic relaxation can be found in the paper by Papadrakakis (1981).

There are many features and capabilities in SANTOS that make it a very versatile and user-friendly computer program. The code has a user-oriented data input scheme based on a free-field reader with keyword descriptors that allow the user to define a complex problem with very few commands. The material library in SANTOS contains several nonlinear constitutive models that can be used to model many different engineering materials from 
metals to foams. The material model interface is also well documented so that new materials may be easily added. SANTOS has the capability to accept temperature history data from an external source for solving thermal stress problems. If the temperature history changes only in time and is uniform throughout the structure, it can be generated within SANTOS itself. The contact or sliding of two surfaces with friction can also be modeled using SANTOS. Surfaces can open or close as the solution dictates, which allows many physical processes to be realistically modeled. Fixed contact surfaces may be used to join two regions with different mesh discretizations. A code interface (Taylor and Flanagan, 1988) is provided which allows an external, user-generated code to pass data to SANTOS and to access internally computed SANTOS variables. An example of such coupling would be a porous flow code providing a pore pressure field to SANTOS and SANTOS providing updated nodal coordinates and stress components to the external code.

SANTOS resides and is maintained in the Sandia National Laboratories Engineering Analysis Code Access System (SEACAS) (Sjaardema, 1993). The program is designed to work with a separate mesh generation program that produces geometry and connectivity information in the SEACO format (Taylor and Flanagan, 1987). The results from a SANTOS calculation are written in the SEACO format to a separate file for processing by separate graphical post-processing and visualization software. SANTOS is written in standard FORTRAN with any system-dependent coding contained in the SUPES (Red-Horse et al., 1990) utilities package.

In the following sections of this report, a description of the theory and the computational models used in SANTOS are given. A description of the available constitutive models is also provided. Because SANTOS is derived directly from PRONTO, many of the theoretical sections are taken directly from the PRONTO theoretical report. An input guide for use of the program is included along with several sample problems and their solutions. 


\subsection{GOVERNING EQUATIONS}

In this chapter, we present the underlying continuum mechanics concepts necessary to follow the development of the numerical algorithms in the following chapters. Bold face characters denote tensors. The order of the tensor is implied by the context of the equation.

\subsection{Kinematics}

A material point in the reference configuration $B_{0}$ with position vector $\mathbf{X}$ occupies position $\mathbf{X}$ at time $t$ in the deformed configuration $\mathrm{B}$. Hence we write $\mathrm{x}=\chi(\mathrm{X}, \mathrm{t})$. The motion from the original configuration to the deformed configuration shown in Figure 2.1.1 has a deformation gradient $\mathrm{F}$ given by

$$
\mathbf{F}=\frac{\partial \mathbf{x}}{\partial \mathbf{X}}, \quad \operatorname{det}(\mathrm{F})>0
$$

Applying the polar decomposition theorem to $\mathrm{F}$ :

$$
\mathbf{F}=\mathbf{V} \mathbf{R}=\mathbf{R} \mathbf{U}
$$

where $\mathbf{V}$ and $\mathbf{U}$ are the symmetric, positive definite left and right stretch tensors, respectively, and $\mathbf{R}$ is a proper orthogonal rotation tensor. Figure 2.1.1 illustrates the intermediate orientations defined by the two alternate decompositions of $\mathbf{F}$ defined by Equation (2.1.2). The determination of $\mathbf{R}$ as defined by Equation (2.1.2) presents a significant numerical challenge. In Section 3.3 , we describe the incremental algebraic algorithm that we use to determine $\mathbf{R}$.

The velocity of the material point $\mathbf{X}$ is written as $\mathbf{v}=\dot{\mathbf{x}}$ where the superposed dot indicates time differentiation holding the material point fixed. The velocity gradient is denoted by $L$ and may be expressed as

$$
L=\frac{\partial \mathbf{v}}{\partial \mathbf{x}}=\frac{\partial \mathbf{v}}{\partial \mathbf{X}} \frac{\partial \mathbf{X}}{\partial \mathbf{x}}=\dot{\mathbf{F}} \mathbf{F}^{-1}
$$

The velocity gradient can be written in terms of the symmetric (D) and antisymmetric $(W)$ parts, respectively,

$$
\mathbf{L}=\mathbf{D}+\mathbf{W}
$$

Using the right decomposition from Equation (2.1.2) in Equation (2.1.3) gives

$$
\mathbf{L}=\dot{\mathbf{R}} \mathbf{R}^{\mathrm{T}}+\mathbf{R} \dot{\mathbf{U}} \mathbf{U}^{-\mathbf{1}} \mathbf{R}^{\mathrm{T}}
$$

Dienes (1979) denoted the first term on the right-hand side of Equation (2.1.5) by $\Omega$ :

$$
\Omega=\dot{\mathbf{R}} \mathbf{R}^{\mathrm{T}} \text {. }
$$




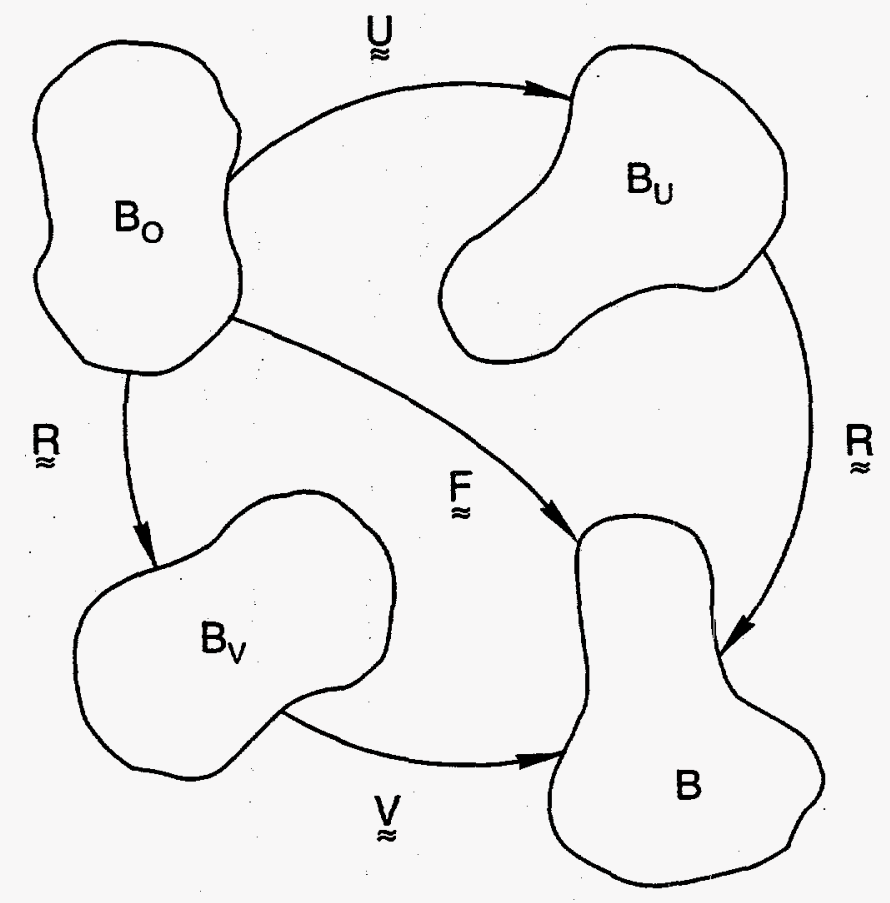

TRI-6348-2-0

Figure 2.1.1. Original, deformed, and intermediate configurations of a body.

Both $W$ and $\Omega$ are antisymmetric and represent a rate of rotation (or angular velocity) about some axes. In general, $\Omega \neq W$. The difference arises when the last term of Equation (2.1.5) is not symmetric. The symmetric part of $\dot{U} \mathbf{U}^{-1}$ is the unrotated deformation rate tensor $\mathbf{d}$ as defined below (note that both $\dot{U}$ and $\mathbf{U}^{-1}$ are symmetric).

$$
\mathbf{d}=\frac{1}{2}\left(\dot{\mathbf{U}} \mathbf{U}^{-1}+\mathbf{U}^{-1} \dot{\mathbf{U}}\right)=\mathbf{R}^{\mathrm{T}} \mathbf{D} \mathbf{R}
$$

There are two possible cases which can cause rotation of a material line element: rigid body rotation and shear. Because total shear vanishes along the axes of principal stretch, the rotation of these axes defines the total rigid body rotation of a material point.

It is a simple exercise in vector analysis to show that Equation (2.1.6) represents the rate of rigid body rotation at a material point as shown by Dienes (1979). It is equally simple to show that $\mathbf{W}$ represents the rate of rotation of the principal axes of the rate of deformation $\mathbf{D}$. Since $\mathbf{D}$ and $\mathbf{W}$ have no sense of the history of deformation, they are not sufficient to define the rate of rotation in a finite deformation context.

Line elements where the rate of shear vanishes rotate solely due to rigid body rotations. These line elements are along the principal axes of $\dot{U}$. We will apply a similar observation below as we derive Dienes' (1979) expression for calculating $\Omega$ : 
Using the left decomposition of Equation (2.1.2) in Equation (2.1.3) gives

$$
\mathbf{L}=\dot{\mathbf{V}} \mathbf{V}^{-1}+\mathbf{V} \Omega \mathbf{V}^{-1}
$$

Postmultiplying by $\mathbf{V}$ yields an expression which defines the decomposition of $L$ into $V$ and $\Omega$ :

$$
\mathbf{L} \mathbf{V}=\dot{\mathbf{V}}+\mathbf{V} \Omega
$$

When the dual vector of the above expression is taken, the symmetric $\dot{\mathbf{V}}$ vanishes to yield a set of three linear equations for the three independent components of $\Omega$.

The antisymmetric part of a tensor may be expressed in terms of its dual vector and the permutation tensor $e_{i j k}$. Define the following dual vectors;

$$
\begin{gathered}
\omega_{i}=e_{i j k} \Omega_{j k} \\
w_{i}=e_{i j k} W_{j k} .
\end{gathered}
$$

Using Equations (2.1.4), (2.1.10), and (2.1.11) in Equation (2.1.9) results in the expression that Dienes (1979) gave for determining $\Omega$ from $W$ and $V$;

$$
\omega=\mathbf{w}-2[\mathbf{V}-\mathbf{I} \operatorname{tr}(\mathbf{V})]^{-1} \mathbf{z}
$$

where

$$
z_{i}=e_{i j k} V_{j m} D_{m k}
$$

We observe from the above expressions that $\Omega=W$ if and only if the product $V D$ is symmetric. This condition requires that the principal axes of the deformation rate $\mathbf{D}$ coincide with the principal axes of the current stretch $\mathbf{V}$. Clearly, a pure rotation is a special case of this condition since $D$, and consequently Equation (2.1.13), vanish.

\subsection{Stress and Strain Rates}

Our constitutive model architecture is posed in terms of the conventional Cauchy stress, but we adopt the approach of Johnson and Bammann (1984) and define a Cauchy stress in the unrotated configuration. The reader seeking more detail than is presented here should see Flanagan and Taylor (1987). The "true" stress in the deformed configuration is denoted by $\mathbf{T}$. The Cauchy stress in the unrotated configuration is denoted by $\sigma$. These two stress measures are related by

$$
\sigma=\mathbf{R}^{\mathrm{T}} \mathbf{T} \mathbf{R}
$$

Each material point in the unrotated configuration has its own reference frame which rotates such that the deformation in this frame is a pure stretch. Then $T$ is simply the tensor $\sigma$ in the fixed global reference frame. The conjugate strain rate measures to $T$ and $\sigma$ are $D$ and $d$, respectively. These strain rates were defined by Equations (2.1.4) and (2.1.7), respectively. 
conjugate strain rate measures to $\mathbf{T}$ and $\sigma$ are $\mathbf{D}$ and $\mathbf{d}$, respectively. These strain rates were defined by Equations (2.1.4) and (2.1.7), respectively.

The Principal of Material Frame Indifference (or objectivity) stipulates that a constitutive law must be insensitive to a change of reference frame (Truesdell, 1966). This requires that only objective quantities may be used in a constitutive law. An objective quantity is one which transforms in the same manner as the energy conjugate stress and strain rate pair under a superposed rigid body motion. The fundamental advantage of the unrotated stress over the true stress is that the material derivative of $\sigma$ is objective, whereas the material derivative of $\mathbf{T}$ is not.

The Jaumann rate defined below is frequently used in constitutive relationships to resolve the need for an objective rate of Cauchy stress.

$$
\hat{\mathbf{T}}=\dot{\mathbf{T}}-\mathbf{W} \mathbf{T}+\mathbf{T} \mathbf{W}
$$

A similar stress rate, called the Green-Naghdi rate by Johnson and Bammann (1984) can be derived by transforming the rate of the unrotated Cauchy stress to the fixed global frame as follows:

$$
\hat{\sigma}=\mathbf{R} \dot{\sigma} \mathbf{R}^{\mathrm{T}}=\dot{\mathbf{T}}-\mathbf{\Omega} \mathbf{T}+\mathbf{T} \mathbf{\Omega}
$$

The Jaumann rate and the Green-Naghdi rate are very similar in form. The important difference between the two is that the Green-Naghdi rate is kinematically consistent with the rate of Cauchy stress, while the Jaumann rate is not. By this statement we mean that $\hat{\sigma}$ is identical to $\dot{T}$ in the absence of rigid body rotations. It is clear that $\hat{\mathbf{T}}$ need not equal $\dot{T}$ under the same conditions since $W$ need not vanish with rigid body rotations.

The simple shear problem presented by Dienes (1979) serves as an excellent demonstration of the symptoms which can occur due to the deficiency of the Jaumann rate. Figure 2.2.1 shows a body which undergoes the following motion:

$$
\mathbf{x}(\mathrm{t})=\mathbf{X}+\mathrm{kt} \mathbf{Y}, \quad \mathbf{y}(\mathrm{t})=\mathbf{Y}, \quad \mathbf{z}(\mathrm{t})=\mathbf{Z}
$$

Dienes applied a simple linear isotropic hypoelastic material law to both the Jaumann rate (2.2.2) and the GreenNaghdi rate (2.2.3). The analytic solution for the true stresses as a function of time using the Jaumann rate is shown in Figure 2.2.1. The Green-Naghdi rate solution is shown in Figure 2.2.2 and demonstrates a monotonic increase in stress with increasing shear strain, while the Jaumann rate results in a harmonic oscillation of the stress. The reason that the Jaumann rate produces this oscillation in stress is that $\mathbf{W}$ gives a constant rate of rotation for the motion defined by Equation (2.2.4), while $\Omega$ vanishes with time. Clearly, the body experiences rotations which diminish over time, but the Jaumann rate continues to drive the stress convection terms at a constant rate. This leads to the oscillatory behavior of the stresses shown in Figure 2.2.1.

A distinct advantage of the unrotated reference frame is that all constitutive models are cast without regard to finite rotations. This greatly simplifies the numerical implementation of new constitutive models. The rotations of 


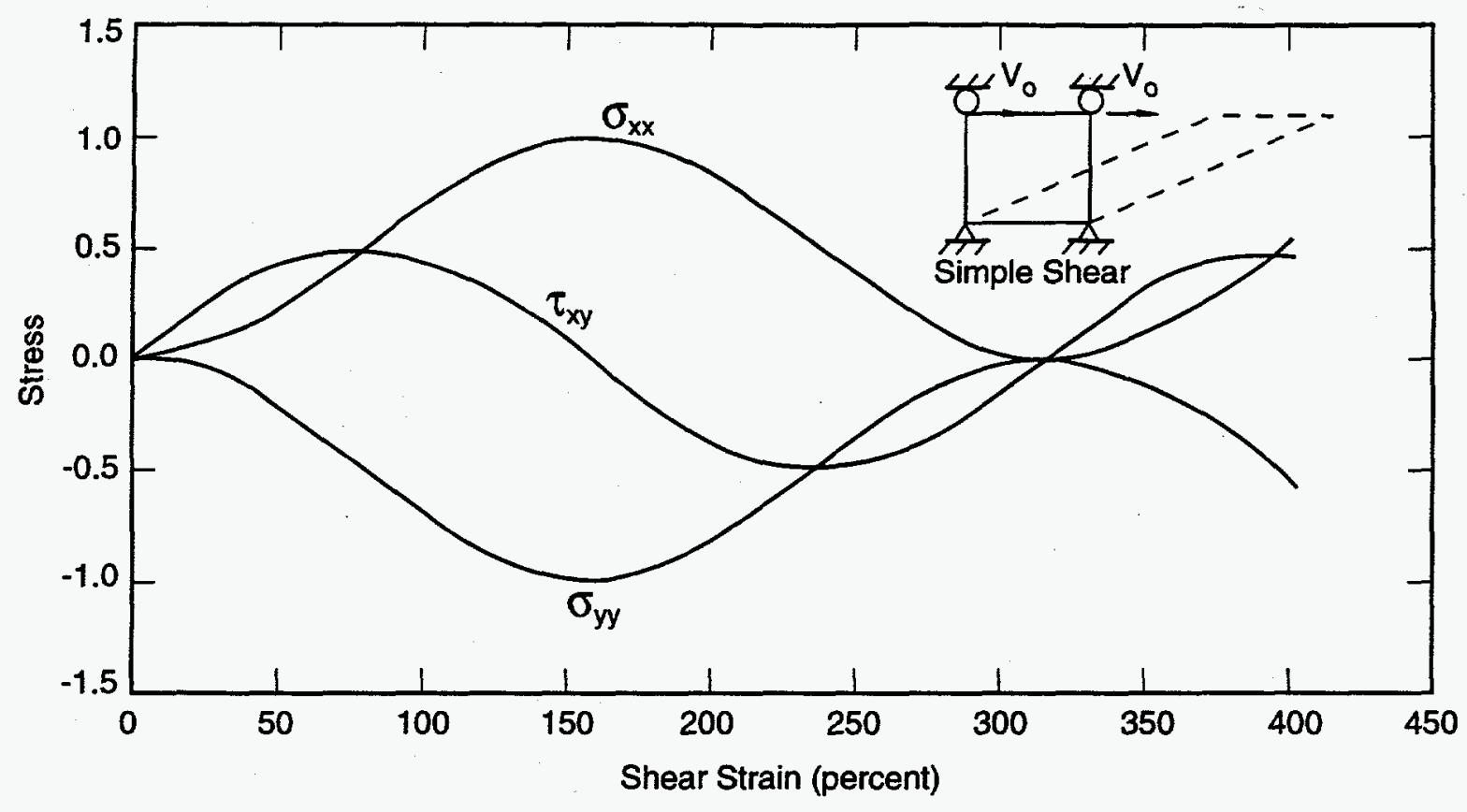

TRI-6348-3-0

Figure 2.2.1. Computed stress-strain curves for a body undergoing simple shear using the Jaumann rate.

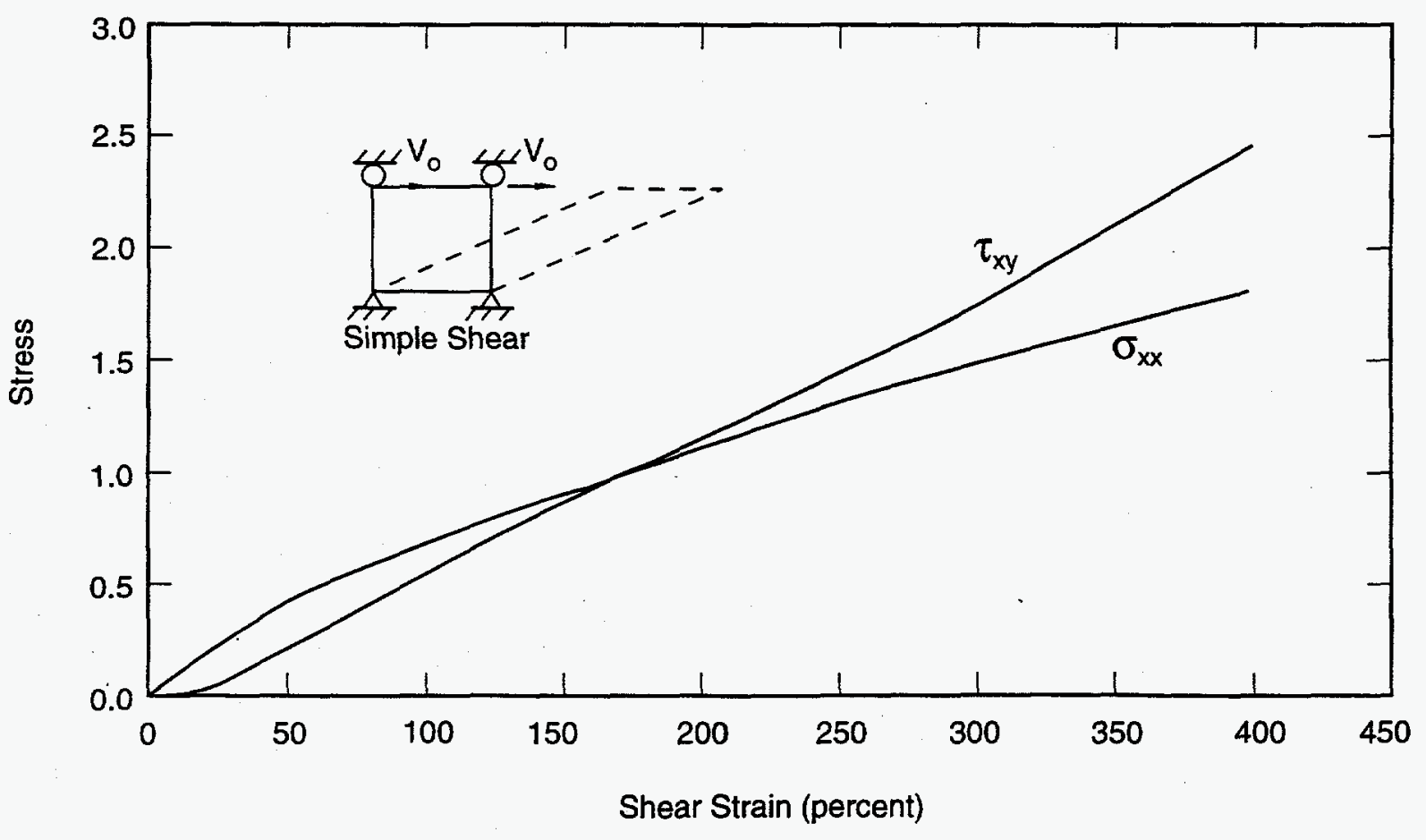

TRI-6348-4-0

Figure 2.2.2. Computed stress-strain curves for a body undergoing simple shear using the Green-Naghdi rate. 
global state variables (e.g., stress and strain) are dealt with on a global level which ensures that all constitutive models are consistent. Internal state variables (e.g., backstress) see no rotations whatsoever.

The drawback to working in the unrotated reference frame is that we must accurately determine the rotation tensor, $\mathbf{R}$, which is not a straightforward numerical calculation. We present an incremental, algebraic algorithm to accomplish this task in Section 3.4.

\subsection{Fundamental Equations}

The equilibrium equation for the body is

$$
\operatorname{div} \mathbf{T}+\rho \mathbf{b}=0
$$

where $\rho$ is the mass density per unit volume and $\mathbf{b}$ is a specific body force vector.

We seek the solution to Equation (2.3.1) subject to the boundary conditions

$$
\mathbf{u}=\mathbf{f}(\mathrm{t}) \text { on } \mathrm{S}_{\mathbf{u}}
$$

where $S_{\mathrm{u}}$ represents the portion of the boundary on which kinematic quantities are specified (displacement). In addition to satisfying the kinematic boundary conditions given by Equation (2.3.2), we must satisfy the traction boundary conditions

$$
\mathbf{T} \bullet \mathbf{n}=\mathbf{s}(\mathrm{t}) \text { on } \mathrm{S}_{\mathrm{T}}
$$

where $\mathrm{S}_{\mathrm{T}}$ represents the portion of the boundary on which tractions are specified. The boundary of the body is given by the union of $S_{u}$ and $S_{T}$, and we note that for a valid mechanics problem $S_{u}$ and $S_{T}$ have a null intersection.

The jump conditions at all contact discontinuities must satisfy the relation

$$
\left(\mathbf{T}^{+}-\mathbf{T}^{-}\right) \bullet \mathbf{n}=0 \text { on } \mathrm{S}_{\mathrm{c}}
$$

where $S_{c}$ represents the contact surface intersection and the subscripts "+" and "-" denote different sides of the contact surface.

To utilize dynamic relaxation as a solution strategy for quasistatics problems, we must first convert the equilibrium equations into equations of motion by adding an acceleration term. Thus,

$$
\operatorname{div} \mathbf{T}+\rho \mathbf{b}=\rho \ddot{\mathbf{u}}
$$

where $\ddot{\mathbf{u}}$ is the acceleration of the material point. Now, all that remains is to introduce the concept of mesh homogenization and artificial damping as well as integrate forward in time from initial conditions until the transient dynamic response has damped out to the static result with equilibrium satisfied. Further description of the implementation of the dynamic relaxation method will be discussed in a later section (Section 3.7). 


\subsection{NUMERICAL FORMULATION}

In this chapter, we describe the finite element formulation of the problem and the numerical algorithms required to perform the spatial and temporal integration of the equations of motion.

\subsection{Four-Node Uniform Strain Element}

The four-node two-dimensional isoparametric element is widely used in computational mechanics. Optimal integration schemes for these elements, however, present a dilemma. A one-point integration of the element underintegrates the element, resulting in a rank deficiency for the element which manifests itself in spurious zero energy modes, commonly referred to as hourglass modes. A two-by-two integration of the element over-integrates the element and can lead to serious problems of element locking in fully plastic and incompressible problems. The fourpoint integration also carries a tremendous computational penalty compared to the one-point rule. We use the onepoint integration of the element and implement an hourglass control scheme to eliminate the spurious modes. The development presented below follows directly from Flanagan and Belytschko (1981). We assume that the reader is somewhat familiar with the finite element method and will not go into a complete description of the method. The reader can consult numerous texts on the method (Hughes, 1987).

The quadrilateral element relates the spatial coordinates $x_{i}$ to the nodal coordinates $x_{i I}$ through the isoparametric shape functions $\phi_{\mathrm{I}}$ as follows:

$$
x_{i}=x_{i I} \phi_{I}(\xi, \eta)
$$

In accordance with indicial notation convention, repeated subscripts imply summation over the range of that subscript. The lowercase subscripts have a range of two, corresponding to the two-dimensional spatial coordinate directions. Uppercase subscripts have a range of four, corresponding to the element nodes.

The same shape functions are used to define the element displacement field in terms of the nodal displacements $\mathrm{u}_{\mathrm{iI}}$

$$
\mathrm{u}_{\mathrm{i}}=\mathrm{u}_{\mathrm{iI}} \phi_{\mathrm{I}}
$$

Since the same shape functions apply to both spatial coordinates and displacements, their material derivative (represented by a superposed dot) must vanish. Hence, the velocity field may be given by

$$
\dot{\mathrm{u}}_{\mathrm{i}}=\dot{\mathrm{u}}_{\mathrm{iI}} \phi_{\mathrm{I}}
$$

and likewise for the acceleration field

$$
\ddot{\mathrm{u}}_{\mathrm{i}}=\ddot{\mathrm{u}}_{\mathrm{iI}} \phi_{\mathrm{I}} .
$$

The velocity gradient tensor, $\mathbf{L}$, is defined in terms of nodal velocities as

$$
\mathrm{L}_{\mathrm{ij}}=\dot{\mathrm{u}}_{\mathrm{i}, \mathrm{j}}=\dot{\mathrm{u}}_{\mathrm{iI}} \phi_{\mathrm{I}, \mathrm{j}}
$$


By convention, a comma preceding a lowercase subscript denotes differentiation with respect to the spatial coordinates (e.g., $\dot{u}_{i, j}$ denotes $\partial \dot{u}_{i} / \partial x_{j}$ ).

The two-dimensional isoparametric-shape functions map the unit square in $\xi-\eta$ to an arbitrary quadrilateral in $x$ $y$, as shown in Figure 3.1.1. We choose to center the unit square at the origin in $\xi-\eta$ space so that the shape functions may be conveniently expanded in terms of an orthogonal set of base vectors, given in Table 3.1, as follows:

$$
\phi_{\mathrm{I}}=\frac{1}{4} \Sigma_{\mathrm{I}}+\frac{1}{2} \xi \Lambda_{1 \mathrm{I}}+\frac{1}{2} \eta \Lambda_{2 \mathrm{I}}+\xi \eta \Gamma_{\mathrm{I}}
$$

Table 3.1

\begin{tabular}{|ccccccc|}
\hline node & $\xi$ & $\eta$ & $\Sigma_{\mathrm{I}}$ & $\Lambda_{1 \mathrm{I}}$ & $\Lambda_{2 \mathrm{I}}$ & $\Gamma_{\mathrm{I}}$ \\
\hline 1 & -.5 & -.5 & 1 & -1 & -1 & 1 \\
2 & .5 & -.5 & 1 & 1 & -1 & -1 \\
3 & .5 & .5 & 1 & 1 & 1 & 1 \\
4 & -.5 & .5 & 1 & -1 & 1 & -1 \\
\hline
\end{tabular}

The above vectors represent the displacement modes of a unit square. The first vector, $\Sigma_{\mathbf{I}}$, accounts for rigid body translation. We call $\Sigma$ the summation vector since it may be employed in indicial notation to represent the algebraic sum of a vector.

The linear base vectors $\Lambda_{\mathrm{iI}}$ may be readily combined to define the uniform normal strains and shear strain in the element. We refer to $\Lambda_{\mathrm{iI}}$ as the volumetric base vectors since, as we will illustrate below, they are the only base vectors that appear in the element area expression.

The last vector, $\Gamma_{\mathrm{I}}$, gives rise to linear strain modes that are neglected in the uniform strain integration. This vector defines the hourglass patterns for a unit cube. The displacement modes represented by the vectors in Table 3.1 are also shown in Figure 3.1.1. 

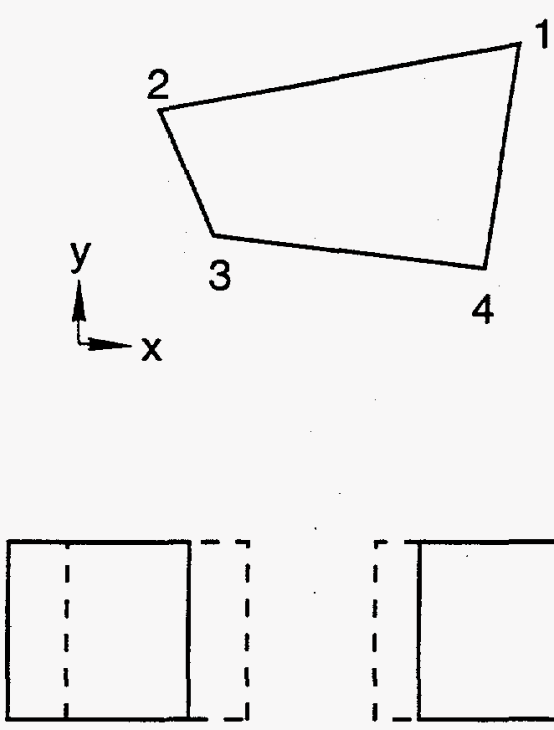

$\Sigma_{\mathrm{I}}$
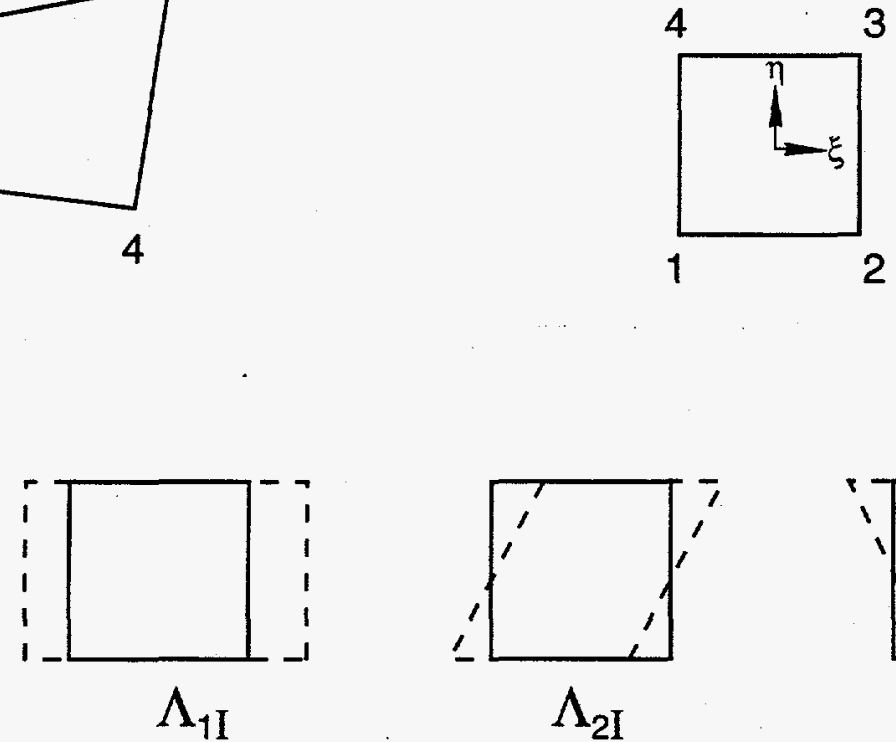

Figure 3.1.1. Mode shapes for the four-node constant strain quadrilateral element.

\subsubsection{Plane Strain Case}

In the finite element method, we replace the momentum Equation (2.3.5) with a weak form of the equation. Using the principal of virtual work, we write the weak form of the equation as

$$
\sum_{e} \int_{V}\left(T_{i j, j}+\rho b_{i}-\rho \ddot{u}_{i}\right) \delta u_{i} d V=0
$$

where $\delta u_{i}$ represents an arbitrary virtual displacement field, with the same interpolation as Equation (3.1.2), which satisfies the kinematic constraints. In plane strain, the thickness of the body is considered uniform and arbitrary and therefore can be eliminated from the preceding expression. Integrating by parts and applying Gauss' divergence theorem to Equation (3.1.7) then gives

$$
\sum_{e}\left[\int_{s} T_{i j} n_{j} \delta u_{i} d_{\ell}-\int_{A_{e}} T_{i j} \delta u_{i, j} d A+\int_{A_{e}} \rho b_{i} \delta u_{i} d A-\int_{A_{e}} \rho \ddot{u}_{i} \delta u_{i} d A\right]=0
$$

The summation symbol represents the assembly of element force vectors into a global nodal force array. We assume that the reader understands the details of this assembly; we will not discuss it further in this document. 
The second integral in the preceding equation is used to define the element internal force vector $f_{i I}$ as

$$
\delta u_{i I} f_{i I}=\int_{A_{e}} T_{i j} \delta u_{i, j} d A
$$

The first and third integrals define the external force vector, and the fourth integral defines the inertial response.

We perform one-point integration by neglecting the nonlinear portion of the element displacement field, thereby considering a state of uniform strain and stress. The preceding expression is approximated by

$$
\mathrm{f}_{\mathrm{iI}}=\overline{\mathrm{T}}_{\mathrm{ij}} \int_{\mathrm{A}_{\mathrm{e}}} \phi_{\mathrm{I}, \mathrm{j}} \mathrm{dA}
$$

where we have eliminated the arbitrary virtual displacements, and $\bar{T}_{\mathrm{ij}}$ represents the assumed uniform stress tensor. By neglecting the nonlinear displacements, we have assumed that the mean stresses depend only on the mean strains. Mean kinematic quantities are defined by integrating over the element as follows:

$$
\dot{\overline{\mathrm{u}}}_{\mathrm{i}, \mathrm{j}}=\frac{1}{\mathrm{~A}} \int_{\mathrm{V}} \dot{\mathrm{u}}_{\mathrm{i}, \mathrm{j}} \mathrm{dA}
$$

We now define the discrete gradient operator as

$$
\mathrm{B}_{\mathrm{iI}}=\int_{\mathrm{A}} \phi_{\mathrm{I}, \mathrm{i}} \mathrm{dA}
$$

The mean velocity gradient, applying Equation (3.1.5), is given by

$$
\dot{\overline{\mathrm{u}}}_{\mathrm{i}, \mathrm{j}}=\frac{1}{\mathrm{~A}} \dot{\mathrm{u}}_{\mathrm{iI}} \mathrm{B}_{\mathrm{jI}}
$$

Combining Equations (3.1.10) and (3.1.12), we may express the nodal forces by

$$
f_{i I}=\bar{T}_{i j} B_{j I} \text {. }
$$

Computing nodal forces with this integration scheme requires evaluation of the gradient operator and the element area. These two tasks are linked since

$$
x_{i, j}=\delta_{i j}
$$

where $\delta_{\mathrm{ij}}$ is the Kroneker delta. Equations (3.1.1), (3.1.12), and (3.1.15) yield

$$
x_{i I} B_{j I}=\int_{V}\left(x_{i I} \phi_{I}\right), j d A=A \delta_{i j}
$$


Consequently, the gradient operator may be expressed by

$$
B_{\mathrm{iI}}=\frac{\partial \mathrm{A}}{\partial \mathrm{x}_{\mathrm{iI}}}
$$

To integrate the element area in closed form, we use the Jacobian of the isoparametric transformation to transform the integral in $x-y$ space to an integral over the unit square:

$$
A=\int_{-1 / 2}^{+1 / 2} \int_{-1 / 2}^{+1 / 2} J d \eta d \xi
$$

where

$$
J=\frac{\partial x}{\partial \xi} \frac{\partial y}{\partial \eta}-\frac{\partial x}{\partial \eta} \frac{\partial y}{\partial \xi}
$$

Therefore, Equation (3.1.18) can be written as

$$
A=x_{I} y_{J} C_{I J}
$$

where

$$
C_{\mathrm{IJ}}=\int_{-1 / 2}^{1 / 2} \int_{-1 / 2}^{1 / 2}\left(\frac{\partial \phi_{\mathrm{I}}}{\partial \xi} \frac{\partial \phi_{\mathrm{J}}}{\partial \eta}-\frac{\partial \phi_{\mathrm{I}}}{\partial \eta} \frac{\partial \phi_{\mathrm{J}}}{\partial \xi}\right) \mathrm{d \eta} \mathrm{d} \xi
$$

In light of Equation (3.1.6), the above integration involves at most bilinear functions. Therefore, only the constant term does not vanish and the integration yields

$$
\mathrm{C}_{\mathrm{IJ}}=\frac{1}{4}\left(\Lambda_{1 \mathrm{I}} \Lambda_{2 \mathrm{~J}}-\Lambda_{2 \mathrm{I}} \Lambda_{\mathrm{IJ}}\right)
$$

Note that $\mathrm{C}_{\mathrm{IJ}}$ is antisymmetric:

$$
\mathrm{C}_{\mathrm{IJ}}=-\mathrm{C}_{\mathrm{IJ}}
$$

Evaluating Equation (3.1.22), we obtain the following explicit representation for $\mathrm{C}_{\mathrm{IJ}}$ :

$$
\mathrm{C}_{\mathrm{IJ}}=\frac{1}{2}\left[\begin{array}{rrrr}
0 & 1 & 0 & -1 \\
-1 & 0 & 1 & 0 \\
0 & -1 & 0 & 1 \\
1 & 0 & -1 & 0
\end{array}\right]
$$

Substituting the above expression into Equation (3.1.20), we obtain the familiar expression for the area of a quadrilateral: 


$$
A=\frac{1}{2}\left[\left(x_{3}-x_{1}\right)\left(y_{4}-y_{2}\right)+\left(x_{2}-x_{4}\right)\left(y_{3}-y_{1}\right)\right]
$$

Using this result in Equation (3.1.17), the B matrix may be expressed as

$$
B_{i J}=C_{I J}\left\{\begin{array}{r}
y_{j} \\
-x_{j}
\end{array}\right\}=\frac{1}{2}\left[\begin{array}{l}
\left(y_{2}-y_{4}\right)\left(y_{3}-y_{1}\right)\left(y_{4}-y_{2}\right)\left(y_{1}-y_{3}\right) \\
\left(x_{4}-x_{2}\right)\left(x_{1}-x_{3}\right)\left(x_{2}-x_{4}\right)\left(x_{3}-x_{1}\right)
\end{array}\right] \text {. }
$$

The mean stress approach used here gives the same result in two dimensions as the one-point quadrature rule for the quadrilateral because the Jacobian is at most bilinear.

\subsubsection{Axisymmetric Case}

The axisymmetric quadrilateral poses a special problem for the finite element method in that we must reduce a three-dimensional variational Equation (3.1.7) to a two-dimensional element domain. The formulation is complicated by the fact that the variational principle is cast in cylindrical, rather than Cartesian coordinates.

We will start by defining the cylindrical coordinate system as follows:

$$
r^{\alpha}=(r, z, \theta)
$$

While the above ordering of the coordinates is unconventional (and not right-handed), it degrades cleanly to the axisymmetric case. Note that Greek indices have a range of three and that superscripts and subscripts indicate contravariant and covariant tensor components, respectively.

The shape functions of the axisymmetric uniform strain quadrilateral are the same as those for the plane strain case (Table 3.1) and are defined implicitly in terms of the nodal coordinates

$$
r_{i}=r_{i I} \phi_{I}
$$

Note that lowercase English indices have a range of two and that, since the two-dimensional coordinate system is Cartesian, there is no distinction between covariant and contravariant tensor components.

In our Lagrangian formulation, the same shape functions are applied to the displacement fields. This implies that the material derivatives of the shape functions vanish. As a result, these shape functions also apply to the velocity field, just as in the plane strain case:

$$
\dot{\mathrm{r}}_{\mathrm{i}}=\dot{\mathrm{r}}_{\mathrm{iI}} \phi_{\mathrm{I}}
$$

The weak form given by Equation (3.1.7) is expressed in cylindrical coordinates as

$$
\int_{V_{e}}\left(\left.T^{\alpha \beta}\right|_{, \beta}+\rho b^{\alpha}-\rho \ddot{u}^{\alpha}\right) \delta u_{\alpha} d V=0
$$


We are now faced with a three-dimensional variational principle, but only a two-dimensional element. Because the differential of volume imposes a factor of $r$ on the differential of area ( $d V=2 \pi \mathrm{dA})$, there is an implicit $r$ weighting on the integrand of the weak form in Equation (3.1.30). This means that the integrand vanishes near the axis of symmetry $(r=0)$ regardless of the variations! This also means that the discretized equations generated by the finite element method become ill-conditioned near the axis.

This difficulty is resolved by dividing the integrand of Equation (3.1.30) by $r$ to reduce the integration to the element domain. However, we must carry this weighting factor in order to apply Gauss' theorem in three dimensions. This technique was referred to as a Petrov-Galerkin, or area-weighted finite element, formulation by Goudreau and Hallquist (1982).

$$
\sum_{e}\left[\left.\frac{1}{2 \pi} \int_{V_{e}}^{T^{\alpha \beta}}\right|_{\beta}\left(\frac{1}{r} \delta u_{\alpha}\right) d V+\int_{A_{e}} \rho b^{\alpha} \delta u_{\alpha} d A-\int_{A_{e}} \rho \ddot{u ̈}^{\alpha} \delta u_{\alpha} d A\right]=0
$$

Integrating by parts and applying Gauss' theorem yields the following:

$$
\sum_{e}\left[\int_{S_{e}} T^{\alpha \beta} \delta u_{\alpha} n_{\beta} d s-\left.\int_{A_{e}} T^{\alpha \beta}\left(\frac{1}{r} \delta u_{\alpha}\right)\right|_{\beta} r d A+\int_{A_{e}} \rho b^{\alpha} \delta u_{\alpha} d A-\int_{A_{e}} \rho \ddot{u}^{\alpha} \delta u_{\alpha} d A\right]=0
$$

Evaluating the covariant derivative in the preceding equation yields

$$
\begin{aligned}
\left(\frac{1}{r} \delta u_{\alpha}\right)_{\beta} & =\left(\frac{1}{r} \delta u_{\alpha}\right), \beta-\Gamma_{\alpha \beta}^{\gamma}\left(\frac{1}{r} \delta u_{\gamma}\right) \\
& =\frac{1}{r} \delta u_{\alpha, \beta}-\frac{1}{r} \Gamma_{\alpha \beta}^{\gamma} \delta u_{\gamma}-\frac{1}{r^{2}} \delta_{1 \beta} \delta u_{\alpha}
\end{aligned}
$$

where $\Gamma_{\alpha \beta}^{\gamma}$ are the Euclidian Christoffel symbols associated with the cylindrical coordinate system. The only nonzero components are

$$
\begin{gathered}
\Gamma_{33}^{1}=-r \\
\Gamma_{13}^{3}=\cdot \Gamma_{31}^{3}=\frac{1}{r}
\end{gathered}
$$

We are now in a position to degenerate the variational equations to the axisymmetric case. The axisymmetry conditions require that variations and derivatives in $\theta$ vanish. Combining Equations (3.1.32) to (3.1.34) and enforcing axisymmetry gives 


$$
\begin{array}{r}
\sum_{e}\left[\int_{S_{e}} T_{i j} n_{j} \delta u_{i} d S-\int_{A_{e}}\left(T_{i j} \delta u_{i, j}+r T^{33} \delta u_{1}-\frac{1}{r} T_{i l} \delta u_{i}\right) d A\right. \\
\left.+\int_{A_{e}} \rho b_{i} \delta u_{i} d A-\int_{A_{e}} \rho \ddot{u}_{i} \delta u_{\xi} d A\right]=0 .
\end{array}
$$

Note that we have dropped the contravariant superscript notation for English indices in going from Equations (3.1.32) to (3.1.35) because as we stated previously, there is no distinction between contravariant and covariant components in our two-dimensional coordinate system.

A byproduct of the Petrov-Galerkin formulation is that the resulting weak form for the axisymmetric case, Equation (3.1.35), is nearly identical to that of the plane strain case, Equation (3.1.8). The only difference is the addition of the last two terms to the internal force expression, which is the second integral above. This is clearly a major architectural advantage to SANTOS.

Note that the last term of the axisymmetric internal force expression is not associated with strain. These forces are analogous to the covected force term which appears in the stress divergence as shown below.

$$
\begin{aligned}
\left.T^{\alpha \beta}\right|_{\beta} & =T^{\alpha \beta}{ }_{\beta}+\Gamma_{\gamma \beta}^{\alpha} T^{\gamma \beta}+\Gamma_{\gamma \beta}^{\beta}+T^{\alpha \gamma} \\
& =T^{\alpha \beta}{ }_{, \beta}+\Gamma_{\gamma \beta}^{\alpha} T^{\gamma \beta}+\frac{1}{r} T^{\alpha 1} .
\end{aligned}
$$

If the $1 / \mathrm{r}$ correction is omitted in Equation (3.1.31), the final term in the axisymmetric internal force disappears.

It is convenient for a finite element program to work with physical, rather than tensoral, stress components. In our formulation, the hoop stress is the only component which requires such a distinction. The physical hoop stress $T_{33}$ is given by

$$
\mathrm{T}_{33}=\mathrm{r}^{2} \mathrm{~T}_{33}
$$

The internal forces are then given by

$$
f_{i I}=\int_{A} T_{i j} \phi_{I, j} d A+\int_{A}\left(T_{33} \delta_{i 1}-T_{i 1}\right) \frac{1}{r} \phi_{I} d A
$$

Evaluating all these integrals with single-point integration yields

$$
f_{i I}=\bar{T}_{i j} B_{j I}+\left(T_{33} \delta_{i 1}-T_{i 1}\right) \frac{A}{4 \bar{r}} \Sigma_{I}
$$

where

$$
\overrightarrow{\mathrm{r}}=\frac{1}{4} \Sigma_{\mathrm{r}_{\mathrm{I}}}
$$


We now see that the internal force vector for the axisymmetric case, Equation (3.1.39), is the same as that for the plane strain case, Equation (3.1.14), with the addition of the hoop stress and covected forces.

The velocity gradient in cylindrical coordinates is

$$
\left.\dot{\mathrm{u}}_{\alpha}\right|_{\beta}=\dot{\mathrm{u}}_{\alpha, \beta}-\Gamma_{\alpha \beta}^{\gamma} \dot{\mathrm{u}}_{\gamma} .
$$

Substituting Equation (3.1.34) into the above equation and enforcing axisymmetry leaves only five nonzero components: the four in-plane components, and the physical hoop strain rate $D_{33}$. This additional strain rate component is defined conjugate to Equation (3.1.37) as

$$
D_{33}=\frac{1}{r^{2}} \dot{\mathrm{u}}_{313}=\frac{\dot{\mathrm{u}}_{1}}{\mathrm{r}} \text {. }
$$

We evaluate this quantity with one-point integration as follows:

$$
\overline{\mathrm{D}}_{33}=\frac{\dot{\mathrm{u}}_{1}}{\overline{\mathrm{r}}}
$$

where $\overline{\mathbf{r}}$ is given by Equation (3.1.40) and

$$
\dot{\overline{\mathrm{u}}}_{1}=\frac{1}{4} \Sigma_{\mathrm{I}} \dot{\mathrm{u}}_{1 \mathrm{I}} .
$$

\subsubsection{Lumped Mass Matrix}

One of the aforementioned advantages of using the Petrov-Galerkin method for the axisymmetric case is that the inertial terms in the variational statement of the boundary value problem are identical for both the plane strain, Equation (3.1.8), and axisymmetric, Equation (3.1.35), cases. Therefore, we can treat both cases at one time.

To reap the benefits of an explicit architecture, we must diagonalize the mass matrix. We do this by integrating the inertial energy variation as follows:

$$
\int_{A} \rho u_{i} \delta u_{i} d A=\ddot{u}_{i I} m_{U} \delta u_{i J}
$$

where

$$
\mathrm{m}_{\mathrm{IJ}}=\rho A \delta_{\mathrm{IJ}}
$$

and $\delta_{\mathrm{IJ}}$ is the Kroneker delta. Clearly, the assembly process for the global mass matrix from the individual element matrices results in a global mass matrix which is diagonal and can be expressed as a vector, $\mathbf{M}_{\mathbf{I}}$. 


\subsection{Explicit Time Integration}

SANTOS uses a modified central difference scheme to integrate the equations of motion through time. By this we mean that the velocities are integrated with a forward difference, while the displacements are integrated with a backward difference. The integration scheme for a node is expressed as

$$
\begin{array}{r}
\ddot{u}_{t}=\left(f_{t}^{E X T}-f_{t}^{I N T}\right) / M \\
\dot{u}_{t+\Delta t}=\dot{u}_{t}+\Delta t \ddot{u}_{t}
\end{array}
$$

and

$$
u_{t+\Delta t}=u_{t}+\Delta t \dot{u}_{t+\Delta t}
$$

where $f_{t}^{E X T}$ and $f_{t}^{I N T}$ are the external and internal nodal forces, respectively, $M$ is the nodal point lumped mass, and $\Delta t$ is the time increment.

The central difference operator is conditionally stable. It can be shown that the Courant stability limit for the operator is given in terms of the highest eigenvalue in the system $\left(\omega_{\max }\right)$ :

$$
\Delta \mathrm{t} \leq \frac{2}{\omega_{\max }}
$$

In Section 3.5, we discuss how the highest eigenvalue is approximated and how we determine a stable time increment.

\subsection{Finite Rotation Algorithm}

We stated in Section 2.2 that one of our fundamental numerical challenges in the development of an accurate algorithm for finite rotations was the determination of $\mathbf{R}$, the rotation tensor defined by the polar decomposition of the deformation gradient $\mathbf{F}$. We developed an incremental algorithm for reasons of computational efficiency and numerical accuracy. The validity of the unrotated reference frame is based on the orthogonal transformation given by Equation (2.2.1). Therefore, the crux of integrating Equation (2.1.6) for $\mathbf{R}$ is to maintain the orthogonality of $\mathbf{R}$. If one integrates $\dot{R}=\Omega \mathbf{R}$ via a forward difference scheme, the orthogonality of $\mathbf{R}$ degenerates rapidly no matter how fine the time increments. We instead adapted the algorithm of Hughes and Winget (1980) for integrating incremental rotations as follows.

A rigid body rotation over a time increment $\Delta t$ may be represented by

$$
\mathbf{x}_{\mathrm{t}+\Delta \mathrm{t}}=\mathbf{Q}_{\Delta \mathrm{t}} \mathbf{x}_{\mathrm{t}}
$$

where $\mathbf{Q}_{\Delta t}$ is a proper orthogonal tensor with the same rate of rotation as $\mathbf{R}$ given by Equation (2.1.6). The total rotation $\mathbf{R}$ is updated via the highly accurate expression below. 


$$
\mathbf{R}_{\mathrm{t}+\Delta \mathrm{t}}=\mathbf{Q}_{\Delta \mathrm{t}} \mathbf{R}_{\mathrm{t}}
$$

For a constant rate of rotation, the midpoint velocity and the midpoint coordinates are related by

$$
\frac{1}{\Delta t}\left(\mathbf{x}_{t+\Delta t}-\mathbf{x}_{t}\right)=\frac{1}{2} \Omega\left(\mathbf{x}_{t+\Delta t}+\mathbf{x}_{t}\right)
$$

Combining Equations (3.3.1) and (3.3.3) yields

$$
\left(\mathbf{Q}_{\Delta t}-\mathbf{I}\right) \mathbf{x}_{\mathrm{t}}=\frac{\Delta \mathrm{t}}{2} \Omega\left(\mathbf{Q}_{\Delta \mathrm{t}}+\mathbf{I}\right) \mathbf{x}_{\mathrm{t}}
$$

Since $\mathbf{x}_{\mathrm{t}}$ is arbitrary in Equation (3.3.4), it may be eliminated. We then solve for $\mathbf{Q}_{\Delta t}$. The result is

$$
\mathbf{Q}_{\Delta \mathrm{t}}=\left(\mathbf{I}-\frac{\Delta \mathrm{t}}{2} \Omega\right)^{-1}\left(\mathbf{I}+\frac{\Delta \mathrm{t}}{2} \Omega\right)
$$

The accuracy of this integration scheme is dependent on the accuracy of the midpoint relationship of Equation (3.3.3). The rate of rotation must not vary significantly over the time increment. Furthermore, Hughes and Winget (1980) showed that the conditioning of Equation (3.3.5) degenerates as $\Delta t \Omega$ grows.

Our complete numerical algorithm for a single time step is as follows:

1. Calculate $\mathbf{D}$ and $\mathbf{W}$.

2. Compute $z_{i}=e_{i j k} V_{j m} D_{m k}$,

$$
\begin{aligned}
& \omega=\mathbf{w}-2[\mathbf{V}-\mathbf{I} \operatorname{tr}(\mathbf{V})]^{-1} \mathbf{z} \text {, and } \\
& \Omega_{\mathrm{ij}}=\frac{1}{2} \mathrm{e}_{\mathrm{ijk}} \omega_{\mathrm{k}} .
\end{aligned}
$$

3. Solve $\quad\left(\mathbf{I}-\frac{\Delta t}{2} \Omega\right) \mathbf{R}_{t+\Delta t}=\left(\mathbf{I}+\frac{\Delta t}{2} \Omega\right) \mathbf{R}_{t}$.

4. Calculate $\dot{\mathbf{V}}=(\mathbf{D}+\mathrm{W}) \mathrm{V}-\mathrm{V} \Omega$.

5. Update $\mathbf{V}_{\mathrm{t}+\Delta \mathrm{t}}=\mathrm{V}_{\mathrm{t}}+\Delta \mathrm{t} \dot{\mathrm{V}}_{\Delta \mathrm{t}}$.

6. Compute $\mathbf{d}=\mathbf{R}^{\mathrm{T}} \mathbf{D} \mathbf{R}$.

7. Integrate $\dot{\sigma}=\mathbf{f}(\mathbf{d}, \sigma)$.

8. Compute $\mathbf{T}=\mathbf{R} \sigma \mathbf{R}^{\mathbf{T}}$.

This algorithm requires that the tensors $\mathbf{V}$ and $\mathbf{R}$ be stored in memory for each element. 


\subsection{Determination of Effective Moduli}

Algorithms for calculating the stable time increment and hourglass control require dilatational and shear moduli. In SANTOS, we use an algorithm for adaptively determining the effective dilatational and shear moduli of the material.

Because SANTOS uses an explicit integration algorithm, the constitutive response over a time step can be recast a posteriori as a hypoelastic relationship. We approximate this relationship as isotropic. This defines effective moduli, $\hat{\lambda}$ and $\hat{\mu}$ in terms of the hypoelastic stress increment and strain increment as follows:

$$
\Delta \sigma_{\mathrm{ij}}=\Delta \mathrm{t}\left(\hat{\lambda}_{\mathrm{kk}} \delta_{\mathrm{ij}}+2 \hat{\mu} \mathrm{d}_{\mathrm{ij}}\right)
$$

Equation (3.4.1) can be rewritten in terms of volumetric and deviatoric parts as

$$
\Delta \sigma_{\mathrm{kk}}=\Delta \mathrm{t}(3 \hat{\lambda}+2 \hat{\mu}) \mathrm{d}_{\mathrm{kk}}
$$

and

$$
s_{i j}=\Delta t 2 \hat{\mu} \varepsilon_{i j}
$$

where

$$
\mathrm{s}_{\mathrm{ij}}=\Delta \sigma_{\mathrm{ij}}-\frac{1}{3} \Delta \sigma_{\mathrm{kk}} \delta_{\mathrm{ij}}
$$

and

$$
\varepsilon_{i j}=d_{i j}-\frac{1}{3} d_{k k} \delta_{i j}
$$

The effective bulk modulus follows directly from Equation (3.4.2) as

$$
3 \hat{\mathrm{K}}=3 \hat{\lambda}+2 \hat{\mu}=\frac{\Delta \sigma_{\mathrm{kk}}}{\Delta \mathrm{td}_{\mathrm{mm}}}
$$

Taking the inner product of Equation (3.4.3) with itself and solving for the effective shear modulus $2 \hat{\mu}$ gives

$$
2 \hat{\mu}=\sqrt{\frac{S_{i j} S_{i j}}{\Delta t^{2} \varepsilon_{m n} \varepsilon_{m n}}} .
$$

Using the result of Equation (3.4.6) with Equation (3.4.7), we can calculate the effective dilatational modulus $\hat{\lambda}+2 \hat{\mu}$ :

$$
\hat{\lambda}+2 \hat{\mu}=\frac{1}{3}(3 \hat{K}+2 \bullet(2 \hat{\mu}))
$$


If the strain increments are insignificant, Equations (3.4.6) and (3.4.7) will not yield numerically meaningful results. In this circumstance, SANTOS sets the dilatational modulus to an initial estimate, $\lambda_{0}+2 \mu_{0}$. An initial estimate for the dilatational modulus is, therefore, the only parameter which every constitutive model is required to provide to the time step control algorithm.

In a case where the volumetric strain increment is significant but the deviatoric increment is not, the effective shear modulus can be estimated by rearranging Equation (3.4.8) as follows:

$$
2 \hat{\mu}=\frac{1}{2}\left(3\left(\lambda_{o}+2 \mu_{o}\right)-3 \hat{K}\right)
$$

If neither strain increment is significant, SANTOS sets the effective shear modulus to the initial dilatational modulus. The algorithm that SANTOS follows to estimate the effective dilatational and shear moduli is summarized in Table 3.2.

Table 3.2

\begin{tabular}{|cccc|}
\hline$\Delta t \mathrm{~d}_{\mathrm{kk}}>10^{-6}$ & $\Delta \mathrm{t}^{2} \varepsilon_{\mathrm{ij}} \varepsilon_{\mathrm{ij}}>10^{-12}$ & $\hat{\lambda}+2 \hat{\mu}$ & $2 \hat{\mu}$ \\
\hline Yes & Yes & $(3.4 .8)$ & $(3.4 .7)$ \\
Yes & No & $\lambda_{\mathrm{o}}+2 \mu_{\mathrm{o}}$ & $(3.4 .9)$ \\
No & Yes & $\lambda_{\mathrm{o}}+2 \mu_{\mathrm{o}}$ & $(3.4 .7)$ \\
No & No & $\lambda_{\mathrm{o}}+2 \mu_{\mathrm{o}}$ & $\lambda_{0}+2 \mu_{\mathrm{o}}$ \\
\hline
\end{tabular}

\subsection{Determination of the Stable Time Increment}

Flanagan and Belytschko (1984) provided eigenvalue estimates for the uniform strain quadrilateral described in Section 3.1. They showed that the maximum eigenvalue was bounded by

$$
4 \frac{\lambda+2 \mu}{\rho} \frac{B_{i I} B_{i I}}{A^{2}} \geq \omega_{\max }^{2} \geq 2 \frac{\lambda+2 \mu}{\rho} \frac{B_{i I} B_{i I}}{A^{2}} .
$$

Using the effective dilatational modulus from Section 3.4 with the eigenvalue estimates of Equation (3.5.1) allows us to write the stability criteria of Equation (3.2.4) as

$$
\Delta \hat{\mathrm{t}}^{2} \leq \frac{\left(\rho_{\mathrm{o}} \mathrm{A}_{\mathrm{o}}\right) \mathrm{A}}{(\lambda+2 \mu) \mathrm{B}_{\mathrm{iI}} \mathrm{B}_{\mathrm{iI}}} .
$$


The stable time increment is determined from Equation (3.5.2) as the minimum over all elements.

The estimate of the critical time increment given in the preceding equation is for the case where there is no damping present in the system. If we define $\varepsilon$ as the fraction of critical damping in the highest element mode, the stability criterion of Equation (3.5.2) becomes

$$
\Delta \mathrm{t} \leqq \Delta \hat{\mathrm{t}}\left(\sqrt{1+\varepsilon^{2}-\varepsilon}\right)
$$

Conventional estimates of the critical time increment size have been based on the transit time of the dilatational wave over the shortest dimension of an element or zone. For the undamped case, this gives

$$
\Delta \mathrm{t}=\ell / \mathrm{c}
$$

where $\mathrm{c}$ is the dilatational wave speed and $\ell$ is the shortest element dimension.

There are two fundamental and important differences between the time increment limits given by Equations (3.5.2) and (3.5.4). First, our time increment limit is dependent on a characteristic element dimension, which is based on the finite element gradient operator and does not require an ad hoc guess of this dimension. This characteristic element dimension, $\ell$, is defined by inspection of Equation (3.5.2) as

$$
\ell=\mathrm{A} / \sqrt{\mathrm{B}_{\mathrm{iI}} \mathrm{B}_{\mathrm{iI}}} .
$$

Second, the sound speed used in the estimate is based on the current response of the material and not on the original elastic sound speed. For materials that experience a reduction in stiffness due to plastic flow, this can result in significant increases in the critical time increment.

It should be noted that the stability analysis performed at each time step predicts the critical time increment for the next step. Our assumption is that the conservativeness of this estimate compensates for any reduction in the stable time increment over a single time step.

\subsection{Hourglass Control Algorithm}

The mean stress-strain formulation of the uniform strain element considers only a fully linear velocity field. The remaining portion of the nodal velocity field is the so-called hourglass field. Excitation of these modes may lead to severe, unresisted mesh distortion. The hourglass control algorithm described here is taken directly from Flanagan and Belytschko (1981). The method isolates the hourglass modes so that they may be treated independently of the rigid body and uniform strain modes.

A fully linear velocity field for the quadrilateral can be described by

$$
\dot{\mathrm{u}}_{\mathrm{i}}^{\mathrm{lin}}=\dot{\overline{\mathrm{u}}}_{\mathrm{i}}+\dot{\overline{\mathrm{u}}}_{\mathrm{i}, \mathrm{j}}\left(\mathrm{x}_{\mathrm{j}}-\overline{\mathrm{x}}_{\mathrm{j}}\right)
$$


The mean coordinates $\bar{x}_{i}$ correspond to the center of the element and are defined as

$$
\overline{\mathrm{x}}_{\mathrm{i}}=\frac{1}{4} \mathrm{x}_{\mathrm{iI}} \Sigma_{\mathrm{I}}
$$

The mean translational velocity is similarly defined by

$$
\dot{\overline{\mathrm{u}}}_{\mathrm{i}}=\frac{1}{4} \dot{\mathrm{u}}_{\mathrm{iI}} \Sigma_{\mathrm{I}}
$$

The linear portion of the nodal velocity field may be expressed by specializing Equation (3.6.1) to the nodes as follows:

$$
\dot{\mathrm{u}}_{\mathrm{iI}}^{\operatorname{lin}}=\dot{\overline{\mathrm{u}}}_{\mathrm{i}} \Sigma_{\mathrm{I}}+\dot{\overline{\mathrm{u}}}_{\mathrm{i}, \mathrm{j}}\left(\mathrm{x}_{\mathrm{jI}}-\overline{\mathrm{x}}_{\mathrm{j}} \Sigma_{\mathrm{I}}\right)
$$

where $\Sigma_{l}$ is used to maintain consistent index notation and indicates that $\dot{\mathrm{u}}_{\mathrm{i}}$ and $\overline{\mathrm{x}}_{\mathrm{j}}$ are independent of position within the element. From Equations (3.1.16) and (3.6.4) and the orthogonality of the base vectors, it follows that

$$
\dot{\mathrm{u}}_{\mathrm{iI}} \Sigma_{\mathrm{I}}=\dot{\mathrm{u}}_{\mathrm{iI}}^{\operatorname{lin}} \Sigma_{\mathrm{I}}=4 \dot{\overline{\mathrm{u}}}_{\mathrm{i}}
$$

and

$$
\dot{\mathrm{u}}_{\mathrm{iI}} B_{\mathrm{jI}}=\dot{\mathrm{u}}_{\mathrm{iI}}^{\operatorname{lin}} B_{\mathrm{jI}}=A \dot{\overline{\mathrm{u}}}_{\mathrm{i}, \mathrm{j}}
$$

The hourglass field $\dot{u}_{\mathrm{iI}}^{\text {hg }}$ may now be defined by removing the linear portion of the nodal velocity field:

$$
\dot{\mathrm{u}}_{\mathrm{iI}}^{\mathrm{hg}}=\dot{\mathrm{u}}_{\mathrm{iI}}-\dot{\mathrm{u}}_{\mathrm{iI}}^{\operatorname{lin}} \text {. }
$$

Equations (3.6.5) through (3.6.7) prove that $\Sigma_{\mathrm{I}}$ and $\mathrm{B}_{\mathrm{jI}}$ are orthogonal to the hourglass field:

$$
\begin{aligned}
& \dot{\mathrm{u}}_{\mathrm{iI}}^{\mathrm{hg}} \sum_{\mathrm{I}}=0 \\
& \dot{\mathrm{u}}_{\mathrm{iI}}^{\mathrm{hg}} \mathrm{B}_{\mathrm{jI}}=0 .
\end{aligned}
$$

Furthermore, it can be shown that the B matrix is a linear combination of the volumetric base vectors, $\Lambda_{\mathrm{I}}$, so Equation (3.6.9) can be written as

$$
\dot{\mathrm{u}}_{\mathrm{iI}}^{\mathrm{hg}} \Lambda_{\mathrm{I}}=0
$$


Equations (3.6.8) and (3.6.10) show that the hourglass field is orthogonal to all the base vectors in Table 3.1 except the hourglass base vectors. Therefore, $\dot{\mathrm{u}}_{\mathrm{iI}}^{\mathrm{hg}}$ may be expanded as a linear combination of the hourglass base vectors as follows:

$$
\dot{\mathrm{u}}_{\mathrm{iI}}^{\mathrm{hg}}=\frac{1}{2} \dot{\mathrm{q}}_{\mathrm{i}} \Gamma_{\mathrm{I}}
$$

The hourglass nodal velocities are represented by $\dot{q}_{\dot{j}}$ above (the leading constant is added to normalize $\Gamma_{I}$ ). We now define the hourglass-shape vector $\gamma_{I}$ such that

$$
\dot{\mathrm{q}}_{\mathrm{i}}=\frac{1}{2} \dot{\mathrm{u}}_{\mathrm{iI}} \gamma_{\mathrm{I}}
$$

By substituting Equations (3.6.4), (3.6.7), and (3.6.12) into (3.6.11), then multiplying by $\Gamma_{I}$ and using the orthogonality of the base vectors, we obtain the following:

$$
\dot{\overline{\mathrm{u}}}_{\mathrm{iI}} \Gamma_{\mathrm{I}}-\dot{\overline{\mathrm{u}}}_{\mathrm{i}, \mathrm{j}} \mathrm{x}_{\mathrm{jI}} \Gamma_{\mathrm{I}}=\dot{\mathrm{u}}_{\mathrm{iI}} \gamma_{\mathrm{I}}
$$

With the definition of the mean velocity gradient, Equation (3.1.13), we can eliminate the nodal velocities above. As a result, we can compute $\gamma_{I}$ from the following expression:

$$
\gamma_{\mathrm{I}}=\Gamma_{\mathrm{I}}-\frac{1}{\mathrm{~A}} \mathbf{B}_{\mathrm{iI}} \mathbf{x}_{\mathrm{iJ}} \Gamma_{\mathrm{J}}
$$

The difference between the hourglass-base vectors $\Gamma_{I}$ and the hourglass-shape vectors $\gamma_{I}$ is very important. They are identical if and only if the quadrilateral is a parallelogram. For a general shape, $\Gamma_{I}$ is orthogonal to $B_{j I}$ while $\gamma_{I}$ is orthogonal to the linear velocity field $\dot{u}_{i I}^{\prime \prime n}$. While $\Gamma_{I}$ defines the hourglass pattern, $\gamma_{I}$ is necessary to accurately detect hourglassing. Equation (3.6.14) is simple enough for the quadrilateral that it can be written explicitly as

$$
\gamma_{I}=\frac{1}{A}\left[\begin{array}{c}
x_{2}\left(y_{3}-y_{4}\right)+x_{3}\left(y_{4}-y_{2}\right)+x_{4}\left(y_{2}-y_{3}\right) \\
x_{3}\left(y_{1}-y_{4}\right)+x_{4}\left(y_{3}-y_{1}\right)+x_{1}\left(y_{4}-y_{3}\right) \\
x_{4}\left(y_{1}-y_{2}\right)+x_{1}\left(y_{2}-y_{4}\right)+x_{2}\left(y_{4}-y_{1}\right) \\
x_{1}\left(y_{3}-y_{2}\right)+x_{2}\left(y_{1}-y_{3}\right)+x_{3}\left(y_{2}-y_{1}\right)
\end{array}\right]
$$

For the purpose of controlling the hourglass modes, we define generalized forces $Q_{i}$, which are conjugate to $\dot{q}_{i}$ so that the rate of work is

$$
\dot{\mathrm{u}}_{\mathrm{iI}} \mathrm{f}_{\mathrm{iI}}^{\mathrm{hg}}=\mathrm{Q}_{\mathrm{i}} \dot{\mathrm{q}}_{\mathrm{i}}
$$


for arbitrary $\dot{\mathrm{u}}_{\mathrm{iI}}$. Using Equation (3.6.12), it follows that the contribution of the hourglass resistance to the nodal forces is given by

$$
\mathrm{f}_{\mathrm{iI}}^{\mathrm{hg}}=\frac{1}{2} \mathrm{Q}_{\mathrm{i}} \gamma_{\mathrm{I}}
$$

Two types of hourglass resistance are used in SANTOS: artificial stiffness and artificial damping. We express this combination as

$$
\mathrm{Q}_{\mathrm{i}}=\mathrm{Q}_{\mathrm{i}}^{\mathrm{K}}+\mathrm{Q}_{\mathrm{i}}^{\mathrm{V}}
$$

In terms of the tunable stiffness ( $K$ ) and viscosity $(\varepsilon)$ factors, these resistances are given by

$$
\begin{gathered}
\dot{Q}_{i}^{K}=\frac{\kappa}{2} 2 \hat{\mu} \frac{B_{i I} B_{i I}}{A} \dot{q}_{i} \\
Q_{i}^{V}=\varepsilon \sqrt{\max (0,2 \hat{\mu}) m} \dot{\mathrm{q}}_{i} .
\end{gathered}
$$

Note that the stiffness expression must be integrated, which further requires that this resistance be stored in a global array.

Observe that the nodal antihourglass forces of Equation (3.6.17) have the shape of $\gamma_{I}$ rather than $\Gamma_{I}$. This fact is essential since the antihourglass forces should be orthogonal to the linear velocity field, so that no energy is transferred to or from the rigid body and uniform strain modes by the antihourglassing scheme.

We would prefer to use only hourglass stiffness and, in fact, this is what is used for the plane strain case ( $\kappa=.05$ and $\varepsilon=0.0$ ). Unfortunately, the nonstrain terms in the Petrov-Galerkin formulation give rise to an instability which is best stabilized using hourglass viscosity. For the axisymmetric case, values of $\kappa=.01$ and $\varepsilon=.03$ are used.

\subsection{Dynamic Relaxation}

As a solution strategy for quasistatic mechanics problems, dynamic relaxation involves first converting the equilibrium equations into equations of motion by adding an acceleration term, secondly, introducing an artificial damping, and finally, integrating forward in time from initial conditions until the transient dynamic response has damped out to the static result with equilibrium satisfied. To produce the transient dynamic problem, an acceleration term is added to the equilibrium Equation (2.3.1), thus becoming

$$
\operatorname{div} \mathbf{T}+\rho f_{b}-r \frac{\partial^{2} \mathbf{u}}{\partial \tau^{2}}=0
$$

where $\mathbf{u}$ is the displacement of the material point and $\mathrm{r}$ is a spatially varying density selected to minimize the number of iteration steps needed to reach equilibrium. The temporal quantity $\tau$ is a pseudo-time scale connected with the dynamic relaxation process but distinct from real time $t$. The acceleration term is discretized the same way that it would be in a true dynamics calculation. This leads us to write the discrete dynamic system as 


$$
\mathbf{M}(\mathbf{r}) \ddot{\mathbf{q}}=\mathbf{f}^{\mathrm{EXT}}-\mathbf{f}^{\mathrm{INT}}
$$

where $\mathbf{M}(r)$ is the mass matrix, $\ddot{\mathbf{q}}=\ddot{\mathbf{u}}(\mathrm{t}), \mathbf{f}^{\mathrm{INT}}$ is the divergence of the stress field, and $\mathbf{f}$ EXT is the vector of prescribed body forces and surface tractions. The mass matrix is computed using the fictitious density, $r$. This density is different for each element, and it is selected such that the element has the same transit time for a dilatational wave as every other element in the mesh. This process is called mesh homogenization, and it is effective in minimizing the number of iterations for convergence.

At time $t_{n}$, equilibrium is satisfied such that $\mathbf{f}_{n}^{\mathbb{I N T}}=\mathbf{f}_{n}^{\text {EXT }}$. A new solution is initiated by incrementing the load to its value at time $t_{n+1}$. In general, equilibrium will not initially be satisfied so that the force imbalance will be represented by the acceleration term:

$$
\mathbf{M}(\mathbf{r}) \ddot{\mathbf{q}}=\mathbf{f}_{\mathrm{n}+1}^{\mathrm{EXT}}-\mathbf{f}_{\mathrm{n}+1}^{\mathrm{INT}}
$$

Central difference expressions are introduced first for the acceleration in terms of the velocity, $\ddot{\mathbf{u}}$ and then for the velocity in terms of the displacement, $\mathbf{u}$. The resulting equations are

$$
\begin{gathered}
\dot{\mathbf{u}}_{\tau+\Delta \tau}=\dot{\mathbf{u}}_{\tau}+\Delta \tau \mathbf{M}(\mathbf{r})^{-1}\left(\mathbf{f}_{\tau}^{\mathrm{EXT}}-\mathbf{f}_{\tau}^{\mathrm{INT}}\right) \\
\mathbf{u}_{\tau+\Delta \tau}=\mathbf{u}_{\tau}+\Delta \tau \dot{\mathbf{u}}_{\tau+\Delta \tau}
\end{gathered}
$$

The dynamic relaxation algorithm is based on these two expressions (Equation (3.7.4). It is a convenient time to introduce the concept of the equilibrium iteration. As the load is incremented to a new value at $t_{n+l}$, the iteration process begins with calculation of the internal forces $f^{I N T}$ and the calculation of the force imbalance. If the force imbalance is greater than a user-specified tolerance, then another iteration through the solution sequence is required. When equilibrium is reached the iteration process stops and new loads are calculated for the next time increment. The central difference expressions above must be solved at each iteration with the appropriate amount of damping to reach the quasistatic solution. These equations take the following form for iteration, $i$, with the self-adaptive damping parameter, $\delta$.

$$
\begin{gathered}
\dot{\mathbf{u}}^{\mathrm{i}+1} \tau+\Delta \tau=\delta \dot{\mathbf{u}}_{\tau}^{\mathrm{i}}+\Delta \tau \delta \mathbf{M}(\mathrm{r})^{-1}\left(\mathbf{f}_{\tau}^{\mathrm{EXT}}-\mathbf{f}_{\tau}^{\mathrm{INT}}\right) \\
\mathbf{u}^{\mathrm{i}+1}{ }_{\tau+\Delta \tau}^{\mathrm{i}}=\mathbf{u}_{\mathrm{t}}^{\mathrm{i}}+\Delta \tau \dot{\mathbf{u}}^{\mathrm{i}} \tau+\Delta \tau
\end{gathered}
$$

Every iteration i leads to a new trial configuration and trial stress state. The path in solution space traced out by the steps is artificial; it is a by-product of the dynamic relaxation, as is the advance in time $\tau$. The trial states $i$ represent equilibrium iterations. Figure 3.7.1 depicts the process in a multidimensional solution space of the nodal point coordinates. The point $\mathrm{n}$ is an equilibrium solution and the point $\mathrm{n}+1$ is the equilibrium state being sought. 


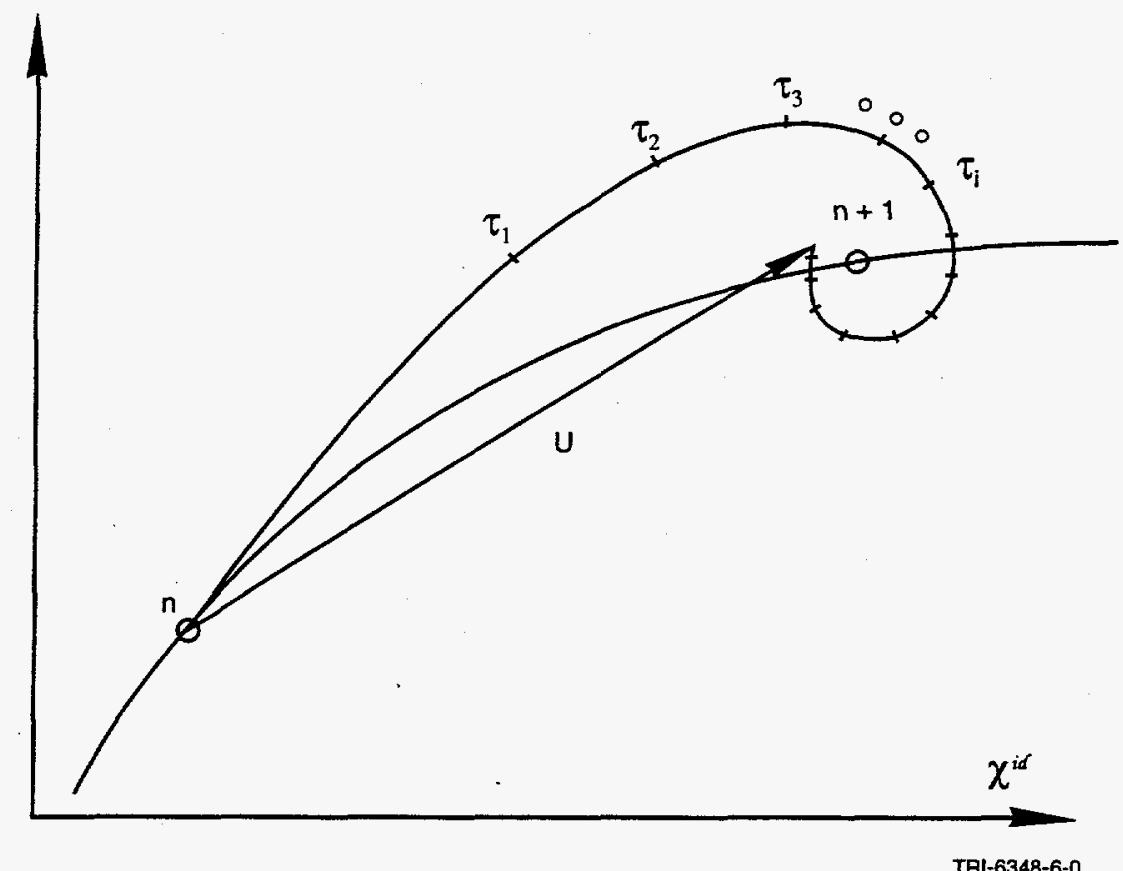

Figure 3.7.1. A model equilibrium iteration sequence in a multi-dimensional configuration space of nodal point positions developed with dynamic relaxation showing convergence at load step $n+1$. The straight line path from $\mathrm{n}$ to the last step calculated from dynamic relaxation is the interval over which the stress is evaluated using the real time step $\Delta t$.

The curved path between $n$ and $n+1$ traces out the true solution. The spiral path marked with the tics and parameterized by steps in $\tau$ is the sequence of trial states generated by the dynamic relaxation method. The straight line from $\mathbf{n}$ to the last step calculated from dynamic relaxation is the interval over which the stress is evaluated using the real time step $\Delta t$. This is an important point in the implementation of the dynamic relaxation scheme. The internal forces $\mathbf{f}^{\mathrm{INT}}$ are re-evaluated at each step $i$ using the trial geometry and when equilibrium is achieved; a straight line approximation to the true path between $n$ and $n+1$ is used for the constitutive model calculations. This scheme uncouples the path dependence and real-time dependence of the constitutive behavior from the arbitrary sequence of trial states generated by the dynamic relaxation method.

Convergence is based on achieving an acceptably small equilibrium imbalance. Because the converged solution is a straight line approximation, the true state at $n+1$ will not be found, but a nearby equilibrium state will be found nonetheless. This truncation error is common to the more conventional finite element methods and can be reduced by decreasing the time step size. The only questions remaining are how to select the variable density $r$, the pseudotime step $\Delta \tau$, and the damping parameter $\delta$ to find a converged solution in the minimum number of steps.

The performance of dynamic relaxation is tied to the minimum natural frequency $\omega_{0}$ and the maximum natural frequency $\omega_{1}$ of the discrete equations. The damping per cycle is frequency dependent. For a given damping factor $\delta$, the decrease in amplitude per cycle is greatest for the lowest frequency component. The damping is then chosen to provide critical damping for the lowest frequency. By looking at the characteristic equation associated with the 
iteration matrix which relates the velocities and displacements at step $n+1$ to those at step $n$, the expression for the damping parameter, $\delta$, is found to be

$$
\delta=1-\left(4 \omega_{0} \omega_{1}\right) /\left(\omega_{0}+\omega_{1}\right)^{2}
$$

The allowable range on $\delta$ is $(0,1)$. A stability analysis on this set of explicit equations produces a critical pseudo-time step given by

$$
\Delta \tau_{c}=2 /\left(\left(\omega_{0}+\omega_{1}\right) \sqrt{\delta}\right)
$$

If the problem is linear so $\omega_{0}$ and $\omega_{1}$ are fixed, then the number of time steps, $N$, required to reduce the vibration amplitude by a factor of ten is

$$
\mathrm{N} \approx 1.15\left(\omega_{1} / \omega_{0}\right)
$$

From this equation, it is seen that any effort to reduce the ratio $\omega_{1} / \omega_{0}$ speeds convergence.

From the linear problem and a uniform mesh of dimension $\Delta x$, the maximum frequency $\omega_{1}$ is given by

$$
\omega_{1}=2 \mathrm{c} / \Delta x=2 / \Delta \tau
$$

In this expression, $\mathrm{c}$ is the dilatational wave speed given by

$$
c=(\lambda+2 \mu) / r
$$

and $r$ is the pseudo-density used for the computation of the fictitious mass. If we substitute the quantity $2 / \Delta \tau$ for 1 and remember that $\omega_{1} \gg \omega_{0}$, then the expression for the damping parameter becomes

$$
\delta=1-2 \omega_{0} \Delta \tau
$$

The fundamental frequency $\omega_{0}$ is continuously estimated using an approximate value found using the Rayleigh Quotient. At each iteration $i$ in the dynamic relaxation scheme, a new estimate $\left(\omega_{0}\right)_{i}$ is computed as

$$
\omega_{0_{i}}=\sqrt{\left(u_{i}^{T} K u_{i}\right) u_{i}^{T} M u_{i}}
$$

where $K$ is a diagonal stiffness matrix whose $\mathrm{j}^{\text {th }}$ component is computed from

$$
K^{j}=\frac{f_{i}^{j I N T}-f_{i-1}^{j I N T}}{\Delta \tau u_{i-1}^{j}} .
$$

With each estimate of the fundamental frequency, a new value of the damping is computed. This has the virtue that the lowest active mode will be found in the event that the fundamental mode is not participating (Underwood, 1983). 


\subsection{Convergence Measures}

When an iterative method, such as dynamic relaxation, is used to solve for static equilibrium, some criterion must be used to determine when the estimated solution is sufficiently close to the actual solution. Convergence of the equilibrium iteration process is achieved when a measure of the problem force imbalance reaches a value less than or equal to a user-supplied error tolerance. The force imbalance is the sum of the external and internal nodal forces which at equilibrium should sum to zero.

In SANTOS, two different convergence error measures are available to the analyst. The first error measure is based on satisfying the following inequality:

$$
\frac{\left\|R_{j}\right\|}{\left\|F_{n}\right\|} \leq \text { TOL }
$$

where $\left\|^{\circ}\right\|$ denotes the $L_{2}$ norm of a vector, $R_{j}$ is the residual or imbalance force vector at iteration $j$, and $F_{n}$ is the external force vector at step $\mathrm{n}$ which is composed of applied tractions, body forces (gravity forces), thermal forces, and the reactions at nodes where zero displacement boundary conditions are applied. Equation 3.8.1 is a measure of how close the problem is to a state of equilibrium. The quantity TOL is input by the analyst as a means of identifying the relative imbalance the analyst is willing to accept in the solution. In SANTOS, TOL is set by default to a value of 0.5 percent. This error is called the GLOBAL CONVERGENCE measure and is the default error measure.

The second error measure implemented in SANTOS is based on satisfying the error tolerance on a node-by-node basis. This error measure is called the LOCAL CONVERGENCE measure. The rationale for this criterion is that what is an acceptable force imbalance in one portion of the problem may be unacceptable at another location. For example, further reduction of a set of force residuals acting in a region of the problem where the elements are large and stiff may produce only a small change in the element stresses in this region. If, however, the same set of force residuals was present at a different location where the element sizes were much smaller and the material was much more flexible, further reduction of the residuals could produce a large change in the element stresses. To address these concerns, the LOCAL CONVERGENCE error measure is included as an option.

The error measure for each component $i$ and iteration $\mathrm{j}$ of the residual force vector is defined as:

$$
\frac{\left|R_{j}^{i}\right|}{\left|F_{n}^{i}\right|+\sum_{e} \max \left(\left|f_{j}^{i}\right|^{e}, f_{\min }^{e}\right)} \leq \text { TOL }
$$

where $R_{j}^{1}$ is the residual or imbalance force, $F_{n}^{i}$ is the external force, $f_{j}^{1}$ is the internal force, and $f_{\text {min }}$ is the minimum internal force in an element produced by a reference hydrostatic stress state specified by the analyst. The minimum internal force is introduced to ensure that the denominator is never zero and to prevent elements with negligible stresses from controlling the convergence of the problem. The internal force contribution is summed over 
all the elements, e, connected to node i. This error measure is satisfied when each component of the force vector satisfies the criterion. 


\subsection{CONSTITUTIVE MODELS}

One of the primary reasons for developing SANTOS was to take advantage of the many state-of-the-art features available in PRONTO and adapt them to quasistatic mechanics problems. One of those features is the flexible material model interface which allows a constitutive model to be added to the code with minimal effort. The constitutive developer does not have to be familiar with the internal workings of SANTOS but only needs to modify a few well documented subroutines to add a new material model. The material model implementation requires the user to provide entries in a few data statements to define the limits of the internal data structure. The code also requires the constitutive developer to provide estimates of the initial dilatational and shear moduli so that the program can compute an initial stable time step. The material model may contain internal state variables that define the state or evolution of the material. The implementation requires that the developer provide names and any required initialization for the internal state variables. The internal state variable names for each material currently implemented are provided in the User Guide section. These quantities may be individually selected for output to the plotting data base. The final changes to the material model subroutines require the developer to provide names for any necessary input quantities such as Young's modulus or Poisson's ratio. The input names for the material models currently implemented are given in the User Guide section. The code currently contains twelve continuum material models with more models being developed as our applications require them. The models range from purely elastic behavior to time-dependent viscoplastic response.

SANTOS utilizes an indirect solution technique which can require hundreds of thousands of calls to the constitutive model during a complex analysis. Thus, efficient implementation of the constitutive model is a primary concern. Considerable effort has gone into writing each material model subroutine such that the routine vectorizes on a vector supercomputer. The material model routine is written in terms of the unrotated Cauchy stress, $\sigma$, and the deformation rate in the unrotated configuration, $\mathbf{d}$. The basic assumption is that the deformation or strain rate is constant over the step. The deformation rate that is available to the constitutive subroutine is the mechanical strain rate, i.e., any thermal strain rate contribution to the total strain rate has already been removed. During each iteration, the latest kinematic quantities are used to update the stress. Stresses written to the plotting data base are rotated to the current configuration.

\subsection{Integration of the Rate Equations}

The constitutive models are written in a rate form and must be integrated forward at each time step. In SANTOS, a forward Euler or a backward Euler integration of the rate equations is used for many of the constitutive models. The forward Euler integration assumes that

$$
\mathrm{f}^{\mathrm{n}+1}=\mathrm{f}^{\mathrm{n}}+\dot{\mathrm{f}}\left(\mathrm{f}^{\mathrm{n}}\right) \Delta \mathrm{t}
$$

where $f$ is the quantity to be integrated, $n$ refers to the current step for which values of $f$ are available and $n+1$ refers to the next step for which values of $f$ are being sought. The quantity $f$ is defined using the known quantities at step $\mathrm{n}$, and $\Delta \mathrm{t}$ is the time step increment. The forward Euler scheme is simple and computationally efficient but is conditionally stable. The time step size allowed is controlled by a stability criterion that varies with each material model. 
The backward Euler integrator has the following form

$$
\mathrm{f}^{\mathrm{n}+1}=\mathrm{f}^{\mathrm{n}}+\dot{\mathrm{f}}\left(\mathrm{f}^{\mathrm{n}+1}\right) \Delta \mathrm{t}
$$

where the term $\dot{\mathrm{f}}$ is evaluated at step $\mathrm{n}+1$. This solution method is implicit and therefore requires some type of iterative method such as Newton-Raphson to solve for $\mathrm{f}^{\mathrm{n}+1}$. The method is computationally more demanding than forward Euler, but the scheme is unconditionally stable. The only restriction on the time step size is accuracy of the solution.

The time-dependent material models implemented in SANTOS, such as the creep and viscoplastic models, use the forward Euler operator even though the method is conditionally stable. The implementations rely on subincrementation within the global time step, $\Delta t$, to maintain numerical stability. In most instances, the userspecified global solution step, $\Delta t$, is larger than the time step needed for accuracy and stability. Economic considerations do not allow the user to take the number of global solution time steps needed to ensure an accurate and stable solution; therefore, the global solution time step is broken into subincrements for integrating the constitutive model. The size of each subincrement adapts to the change in stress occurring within the global solution step. So although this subincrementation process maintains the direction and magnitude of the total strain increments as constant for the global step, it allows the stress components to change over the step. That is, after each subincremental time step, the stresses and inelastic strain rates as well as the critical time step are updated before computing the solution for the next subincrement.

The implementation of this algorithm is designed to take advantage of the vector architecture of the Cray computer. The constitutive model is called with the total strain rates for the step and the stress from the previous step. Processing is done on a block of 64 elements, one block at a time. There are two FORTRAN loops involved in this approach. The outer loop is an implicit loop that adapts the size of the subincrement as the stresses change within the global solution step. This loop is not vectorizable. The inner loop computes the stresses for a block of NE elements, with NE having a maximum of 64. This loop is vectorizable. An additional feature of this approach, which is unique to indirect solution schemes, is that each element block may have its own unique number of subincrements. Thus, the amount of computation is minimal for elements in regions where the stress is small and the computational effort is concentrated where the stress is largest.

The key to the scheme is the accurate determination of the stable time step which is accomplished using the work of Cormeau (1975) who developed a method for analytically determining the stable time step for a particular constitutive model. To determine the analytical expression for the stable time step size, we introduce the following linearized differential equation

$$
\dot{s}=\dot{\sigma}_{t}+\left.\frac{\partial}{\partial \sigma} \dot{\sigma}\right|_{t}\left(s-\sigma_{t}\right)
$$

where the quantity, $\sigma_{t}$, represents the deviatoric stress at time $t$. This equation represents a first-order Taylor series expansion about the stress state at time $t$. This equation can be rewritten as

$$
\dot{y}+A y=f
$$


where $\mathrm{y}$ is a column vector containing the stress components and $\mathrm{A}$ is a square matrix defined by

$$
A=\left[\frac{\partial}{\partial \sigma}(\dot{\sigma})\right]_{t}
$$

A stability analysis of the forward Euler integrator shows that the time interval is stable if $\Delta \mathrm{t}<\frac{2}{\left|\lambda_{\max }\right|}$ where $\lambda_{\max }$ is the largest eigenvalue of the square matrix A. Once we have the analytic expression for the stable time step, we can write an efficient, vectorized material model subroutine for implementation into SANTOS.

\subsection{Adaptive Time Stepping}

One important feature available for the time-dependent material models in SANTOS is the capability to do adaptable time stepping. This feature is desirable when the mechanics of the problem dictate small time steps during the early stress transient, but the stress reaches a steady state at later times and the analyst desires to use larger time steps. If we consider a function $f(t)$ which is analytic in the neighborhood of a point $t$ :

$$
f(t+h)=f(t)+h f^{\prime}(t)+\frac{h^{2}}{2} f^{\prime \prime}(t)+\frac{h^{3}}{3 !} f^{\prime \prime \prime}(t)+\ldots
$$

The forward Euler method is obtained by taking the first two terms of the series:

$$
f(t+h)=f(t)+h f^{\prime}(t)+O\left(h^{2}\right)
$$

where $O\left(h^{2}\right)$ is the error associated with the truncation. The above equation can be rewritten in a slightly different form:

$$
f(t+h)=f(t)+h f^{\prime}(t)+\frac{h^{2}}{2} f^{\prime \prime}(\xi)
$$

where $t_{i}<\xi<t+h$ or

$$
f_{i+1}=f_{i}+h f_{i i}^{\prime}+\frac{h^{2}}{2} f^{\prime \prime}(\xi)
$$

and $t_{i}<\xi<t_{i+1}$ where the last term is the truncation error per step.

If it is assumed that $\mathrm{f}^{\prime \prime}$ is fairly constant over the $\mathrm{i}^{\text {th }}$ step interval, an estimate of the truncation error $\mathrm{E}_{\mathrm{T}}$ at the $\mathrm{i}^{\text {th }}$ step can be obtained from

$$
E_{T_{i}} \cong \frac{h^{2}}{2} f^{\prime \prime}{ }_{i}
$$

where $\mathrm{f}^{\prime \prime}$ is evaluated at $\xi=t_{i}$. 
The criterion for the time step control is

$$
\left|\mathrm{E}_{\mathrm{T}_{\mathrm{i}}}\right|<\varepsilon\left|\mathrm{f}_{\mathrm{i}}\right|
$$

where $\varepsilon$ is some small number. Replacing $E_{T_{i}}$ in the above expression with $\frac{h^{2}}{2} f_{i}$ " and solving for the time step, $h$, gives the following expression

$$
h<\sqrt{\frac{2 \varepsilon\left|f_{j}\right|}{\left|f^{\prime \prime}{ }_{i}\right|}} .
$$

In SANTOS, we choose to control the time step with the effective stress so that the above equation becomes

$$
h<\sqrt{\frac{2 \varepsilon \bar{\sigma}_{i}}{\bar{\sigma}_{i}^{\prime \prime}}} .
$$

The accuracy of the method depends on the value of $\varepsilon$ chosen. For example, we might restrict the error to 1 percent of $\bar{\sigma}_{i}$ at the beginning of the step so $\varepsilon$ would be selected as 0.01 . Experience has shown that values of $\varepsilon$ in the range $.01-.02$ produce acceptable results.

\subsection{Basic Definitions and Assumptions}

The constitutive models implemented in SANTOS are described in the following sections. The fundamental assumptions used in developing the models are presented along with some details of their implementation. The nomenclature used for the descriptions will be presented first. Several of the models have their descriptions taken from other sources, and we will follow the nomenclature of those sources where appropriate. Throughout the report, components of tensors will appear using indicial notation, $\sigma_{\mathrm{ij}}$, while equivalent scalar quantities appear with a bar, $\bar{\sigma}$.

The material model development makes the fundamental assumption of an additive strain rate decomposition of the total strain rate components, $d_{i j}$, into elastic and inelastic parts.

$$
d_{i j}=d_{i j}^{e l}+d_{i j}^{i n} \text {. }
$$

The resulting stress rate, $\dot{\sigma}_{\mathrm{ij}}$, is determined from the elastic part of the strain rate using Hooke's law

$$
\dot{\sigma}_{\mathrm{ij}}=\mathrm{C}_{\mathrm{ijkl}} \mathrm{d}_{\mathrm{kl}}^{\mathrm{el}}=\mathrm{C}_{\mathrm{ijkl}}\left(\mathrm{d}_{\mathrm{kl}}-\mathrm{d}_{\mathrm{kl}}{ }^{\text {in }}\right)
$$

where $\mathrm{C}$ is a $4^{\text {th }}$ order tensor of Hookean elastic constants. The stress rate can be broken into two independent parts representing volumetric and deviatoric behavior. The volumetric behavior is assumed for most material models to be purely elastic with the volumetric strain rate, $d_{k k}$, linearly related to the pressure, $\dot{p}$, through the relation 


$$
\dot{\mathrm{p}}=\frac{\dot{\sigma}_{\mathrm{kk}}}{3}=\mathrm{Kd} \mathrm{kk}_{\mathrm{k}}
$$

where $\mathrm{K}$ is the bulk modulus of the material. Because the strain rates are assumed constant over the step, the pressure at the end of the step can be easily found from the expression

$$
\mathrm{p}_{\mathrm{n}+1}=\mathrm{p}_{\mathrm{n}}+\mathrm{Kd}_{\mathrm{kk}} \Delta \mathrm{t}
$$

where $\Delta t$ is the time step size and $p_{n}$ is the pressure at the beginning of the step. There are material models in SANTOS that do not have a linear bulk response. These exceptions include the volumetric creep model, soil and crushable foam model, and low density foam model. The particular volumetric response for each of these models will be discussed in each individual section.

The deviatoric stress rate, $\dot{S}_{\mathrm{ij}}$, is computed from the relation

$$
\dot{S}_{i j}=\dot{\sigma}_{i j}-\dot{p} \delta_{i j}
$$

where $\delta_{\mathrm{ij}}$ is the Kronecker delta. If we rewrite the equation for the stress rate in terms of the deviatoric stress part, we have

$$
\dot{S}_{i j}=2 \mu\left(\dot{\varepsilon}_{i j}-\dot{\varepsilon}_{i j}\right)
$$

where $\dot{\varepsilon}_{\mathrm{ij}}$ is the deviatoric component of the strain rate, $\mathrm{d}_{\mathrm{ij}}$. The deviatoric strain rate components are similarly calculated.

$$
\dot{\varepsilon}_{\mathrm{ij}}=\mathrm{d}_{\mathrm{ij}}-\frac{1}{3} \mathrm{~d}_{\mathrm{kk}} \delta_{\mathrm{ij}}
$$

In most of the material models currently implemented in SANTOS, we assume von Mises flow, and we can define the equivalent von Mises stress, $\bar{\sigma}=\sqrt{\frac{3}{2} S_{\mathrm{ij}} \mathrm{S}_{\mathrm{ij}}}$, and the equivalent deviatoric strain rate, $\dot{\bar{\varepsilon}}=\sqrt{\frac{2}{3} \dot{\varepsilon}_{\mathrm{ij}} \dot{\varepsilon}_{\mathrm{ij}}}$. It is convenient to introduce the idea of an elastic "trial" stress state for the end of the time step. This stress state is used in the plasticity models to determine if yielding will occur during the step, and it is also used for the time-dependent models. Given the deviatoric stress state at the beginning of the step, $S_{\mathrm{ij}}^{\mathrm{n}}$, the elastic "trial" stress state for the end of the step is

$$
S_{i j}{ }^{t r}=S_{i j}{ }^{n}+2 \mu \dot{\varepsilon}_{i j} \Delta t
$$

where $\mu$ is the shear modulus, $\Delta t$ is the time step increment, and $\dot{\varepsilon}_{\mathrm{ij}}$ is the deviatoric strain rate. If yielding does not occur during the time step, then the trial stress becomes the final stress state at $S_{i j}^{n+1}$. 


\subsection{Elastic Material, Hooke's Law}

A linear elastic material is defined using Hooke's Law. In a rate form, this is written as

$$
\dot{\sigma}_{\mathrm{ij}}=\lambda\left(\mathrm{d}_{\mathrm{kk}}\right) \delta_{\mathrm{ij}}+2 \mu \mathrm{d}_{\mathrm{ij}}
$$

where $\lambda$ and $\mu$ are the elastic Lamé material constants. The stress rate equation is integrated forward using the backward Euler integrator. The model has no internal state variables.

The PROP array for this material contains the following entries:

PROP(1) - Young's modulus, E

PROP(2) - Poisson's ratio, $v$

PROP(3) - $\lambda$

$\operatorname{PROP}(4)-2 \mu$.

\subsection{Elastic Plastic Material with Combined Kinematic and Isotropic Hardening}

The elastic plastic model is based on a standard von Mises yield condition and uses combined kinematic and isotropic hardening. Isotropic hardening is the behavior where the radius of the yield surface grows equally in all directions due to plastic straining. Kinematic hardening is the behavior where the radius of the yield surface remains constant, but the center of the yield surface translates in the direction of the plastic strain rate. In this discussion of the elastic plastic material model, we assume that the material is yielding and that plastic straining will occur. In the event that yielding does not occur, the material behavior is elastic and the stress is computed using Hooke's Law as described in Section 4.4. This model is widely used in many finite element computer programs, and the current derivation is taken from Taylor and Flanagan (1987).

Some definitions and assumptions are outlined here. Referring to Figure 4.5.1, which shows the yield surface in deviatoric stress space, we define the backstress (the center of the yield surface) by the tensor, $\alpha$.

If $\sigma$ is the current value of the stress, we define the deviatoric part of the current stress by

$$
S=\sigma-\frac{1}{3} \operatorname{tr} \sigma \delta
$$

We define the stress difference measured by subtracting the backstress from the deviatoric stress by

$$
\xi=\sigma-\alpha
$$

The magnitude of the stress difference, $R$, is defined by

$$
\mathbf{R}=|\xi|=\sqrt{\xi: \xi}
$$




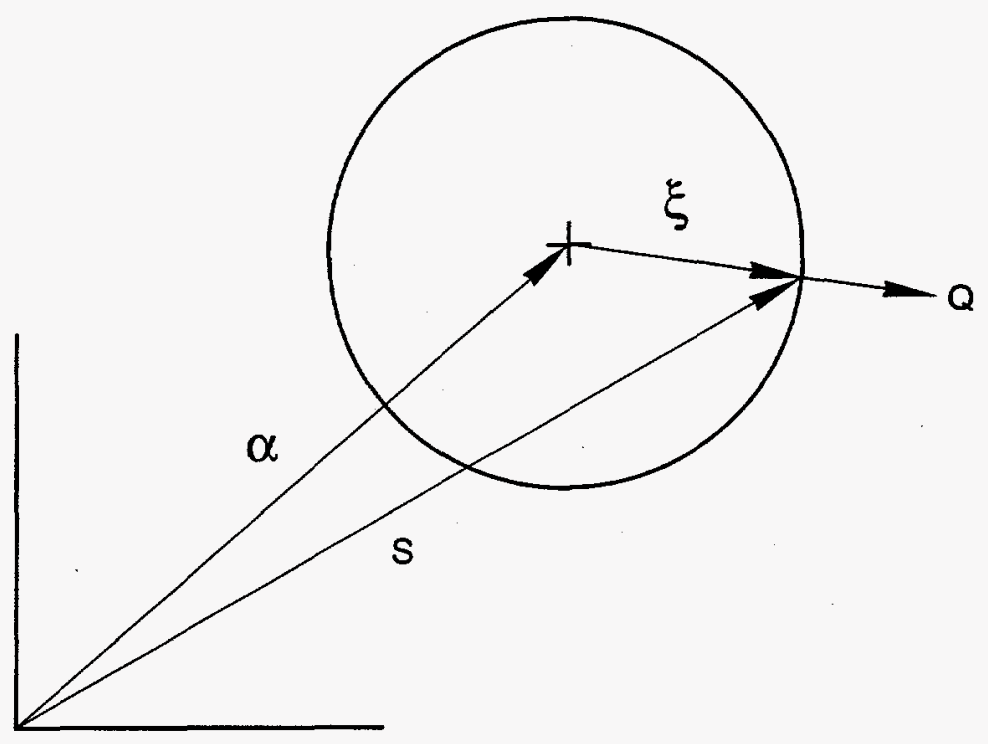

TRI-6348-7-0

Figure 4.5.1. Yield surface in deviatoric stress space.

where we denote the inner product of second order tensors by $\mathbf{S}: \mathbf{S}=\mathrm{S}_{\mathrm{ij}} \mathbf{S}_{\mathrm{ij}}$. Note that if the backstress is zero (isotropic hardening case), the stress difference is equal to the deviatoric part of the current stress, $\mathbf{S}$.

The von Mises yield surface is defined as

$$
f(\sigma)=\frac{1}{2} \xi: \xi=\kappa^{2}
$$

The von Mises effective stress, $\bar{\sigma}$, is defined by

$$
\bar{\sigma}=\sqrt{\frac{3}{2} \xi: \xi} .
$$

Since $\mathrm{R}$ is the magnitude of the deviatoric stress tensor when $\alpha=0$, it follows that

$$
\mathrm{R}=\sqrt{2} \kappa=\sqrt{\frac{2}{3}} \bar{\sigma}
$$

The normal to the yield surface can be determined from Equation (4.5.4)

$$
\mathbf{Q}=\frac{\partial \mathrm{f}}{\partial \sigma} /\left|\frac{\partial \mathrm{f}}{\partial \sigma}\right|=\xi / \mathrm{R}
$$

We assume that the strain rate can be decomposed into elastic and plastic parts by an additive decomposition

$$
d=d^{e l}+d^{p l}
$$


and assume that the plastic part of the strain rate is given by a normality condition

$$
\mathbf{d p l}=\gamma \mathbf{Q}
$$

when the scalar multiplier, $\gamma$, must be determined.

A scalar measure of equivalent plastic strain rate is defined by

$$
\overline{\mathrm{d}}^{\mathrm{pl}}=\sqrt{\frac{2}{3} \mathrm{~d}^{\mathrm{pl}}: \mathrm{d}^{\mathrm{pl}}}
$$

which is chosen such that

$$
\bar{\sigma} \overline{\mathrm{d}}^{\mathrm{pl}}=\sigma: \mathbf{d}^{\mathbf{p l}}
$$

The stress rate is assumed to be purely due to the elastic part of the strain rate and is expressed in terms of Hooke's law by

$$
\dot{\sigma}=\lambda \operatorname{tr} \mathbf{d}^{\mathrm{el}} \delta+2 \mu \mathbf{d}^{\mathrm{el}}
$$

where $\lambda$ and $\mu$ are the Lamé constants for the material.

Below, we develop the theory for the cases of isotropic hardening, kinematic hardening, and combined hardening separately so that the reader can see the details for each case.

\subsubsection{Isotropic Hardening}

In the isotropic hardening case, the backstress is zero and the stress difference is equal to the deviatoric stress, $\mathrm{S}$. We write a consistency condition by taking the rate of Equation (4.5.4)

$$
\dot{f}(\sigma)=2 \kappa \dot{K}
$$

By consistency we mean that the state of stress must remain on the yield surface at all times. We use the chain rule and the definition of the normal to the yield surface given by Equation (4.5.7) to obtain

$$
\dot{\mathrm{f}}(\sigma)=\frac{\partial \mathrm{f}}{\partial \sigma}: \dot{\sigma}=\left|\frac{\partial \mathrm{f}}{\partial \sigma}\right| \mathrm{Q}: \dot{\sigma}
$$

and from Equations (4.5.3) and (4.5.4)

$$
\left|\frac{\partial f}{\partial \sigma}\right|=|\mathbf{S}|=\mathbf{R}
$$

Combining Equations (4.5.13), (4.5.14), and (4.5.15) 


$$
\frac{1}{\mathrm{R}} \mathbf{S}: \dot{\sigma}=\dot{\mathbf{R}}
$$

Because $\mathbf{S}$ is deviatoric, $\mathbf{S}: \dot{\sigma}=\mathbf{S}: \dot{\mathbf{S}}$ and

$$
\mathbf{S}: \dot{\mathbf{S}}=\frac{\mathrm{d}}{\mathrm{dt}}\left(\frac{1}{2} \mathbf{S}: \mathbf{S}\right)=\frac{\mathrm{d}}{\mathrm{dt}}\left(\frac{\bar{\sigma}^{2}}{3}\right)=\frac{2}{3} \bar{\sigma} \dot{\sigma} .
$$

Then Equation (4.5.16) can be written as

$$
\dot{\mathrm{R}}=\sqrt{\frac{2}{3}} \dot{\bar{\sigma}}=\sqrt{\frac{2}{3}} \mathrm{H}^{\prime} \overline{\mathrm{d}}^{\mathrm{pl}}
$$

where $H^{\prime}$ is the slope of the yield stress versus equivalent plastic strain $\left(\bar{\sigma}\right.$ versus $\left.\bar{\varepsilon}^{\mathrm{pl}}\right)$. This is derivable from the data from a uniaxial tension test as shown in Figure 4.5.2.

The consistency condition, Equation (4.5.16) and Equation (4.5.18), result in

$$
\sqrt{\frac{2}{3}} \mathrm{H}^{\prime} \overline{\mathrm{d}}^{\mathrm{pl}}=\mathbf{Q}: \dot{\sigma}
$$

We define the trial elastic stress rate $\dot{\sigma}^{\text {tr }}$ by

$$
\dot{\boldsymbol{\sigma}}^{\mathrm{tr}}=\mathbf{C}: \mathbf{d}
$$
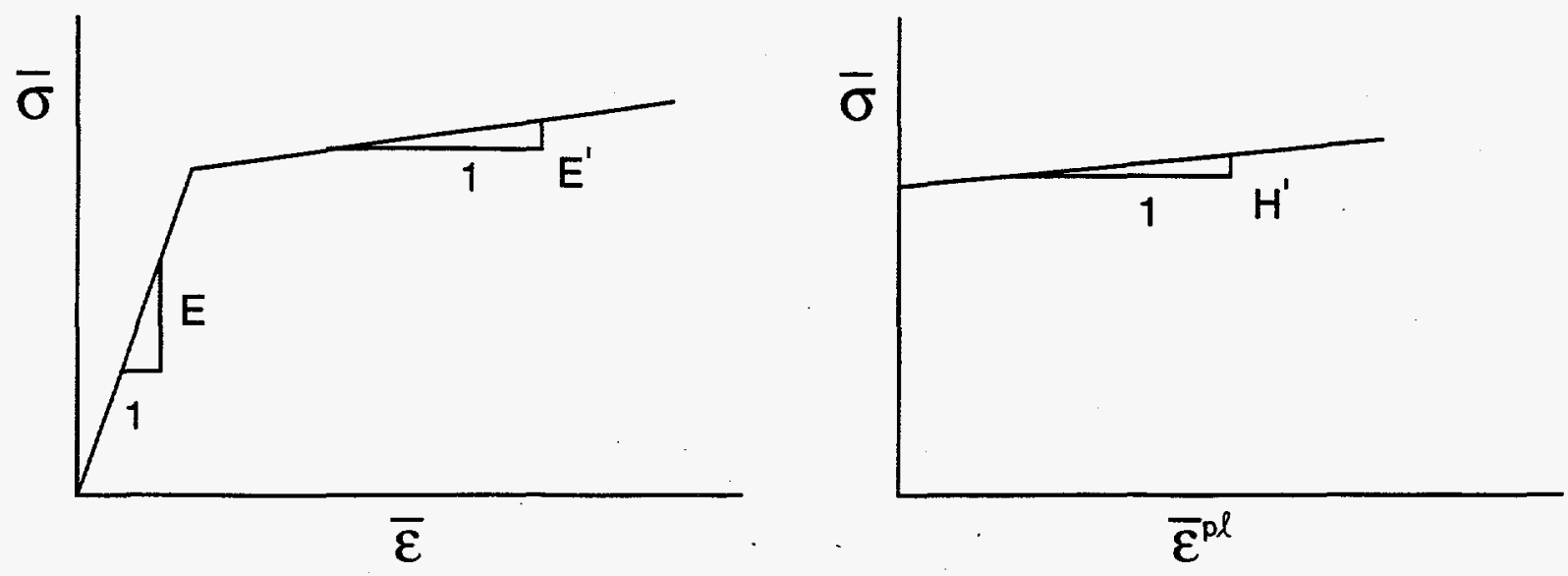

$$
H^{\prime}=\frac{E E^{\prime}}{E-E^{\prime}}
$$

Figure 4.5.2. Conversion of data from a uniaxial tension test to equivalent plastic strain versus von Mises stress. 
where $C$ is the fourth order tensor of elastic coefficients defined by Equation (4.5.12). Combining the strain rate decomposition defined in Equation (4.5.8) with Equations (4.5.19) and (4.5.20) yields

$$
\sqrt{\frac{2}{3}} \mathrm{H}^{\prime} \overline{\mathrm{d}}^{\mathrm{pl}}=\mathbf{Q}: \dot{\boldsymbol{\sigma}}^{\mathrm{tr}}-\mathbf{Q}: \mathbf{C}: \mathbf{d}^{\mathrm{pl}}
$$

We note that because $\mathbf{Q}$ is deviatoric, $\mathbf{C}: \mathbf{Q}=2 \mu \mathbf{Q}$ and $\mathbf{Q}: \mathbf{C}: \mathbf{Q}=2 \mu$. Then using the normality condition, Equation (4.5.9), the definition of equivalent plastic strain, Equation (4.5.10), and Equation (4.5.21)

$$
\frac{2}{3} \mathrm{H}^{\prime} \gamma=\mathbf{Q}: \dot{\sigma}^{\mathrm{tr}}-\gamma 2 \mu
$$

and since $Q$ is deviatoric ( $Q: \dot{\sigma}^{\text {tr }}=2 \mu \mathrm{Q}: \mathbf{d}$ ), we can determine $\gamma$ from Equation (4.5.22) as

$$
\gamma=\frac{1}{\left(1+H^{\prime} / 3 \mu\right)} \mathbf{Q}: \mathbf{d}
$$

The current normal to the yield surface, $\mathbf{Q}$, and the total strain rate, $\mathbf{d}$, are known quantities. Hence, from Equation (4.5.23), $\gamma$ can be determined which can be used in Equation (4.5.9) to determine the plastic part of the strain rate which, with the additive strain rate decomposition and the elastic stress rate of Equations (4.5.8) and (4.5.12), completes the definition of the rate equations.

We still must explain how to integrate the rate equations subject to the constraint that the stress must remain on the yield surface. We will show how that is accomplished in Section 4.5.4.

\subsubsection{Kinematic Hardenịng}

Just as before with the isotropic hardening case, we write a von Mises yield condition but now in terms of the stress difference

$$
f(\xi)=\frac{1}{2} \xi: \xi=\kappa^{2}
$$

It is important to remember that $\alpha$ and $\xi$ are deviatoric tensors. The consistency condition is written for kinematic hardening as

$$
\dot{\mathrm{f}}(\xi)=0
$$

because the size of the yield surface does not grow with kinematic hardening; therefore, $\dot{\kappa}=0$. Using the chain rule on Equation (4.5.25)

$$
\frac{\partial \mathrm{f}}{\partial \xi}: \dot{\xi}=0
$$


and

$$
\frac{\partial \mathrm{f}}{\partial \xi}=\left|\frac{\partial \mathrm{f}}{\partial \xi}\right| \mathbf{Q}=\mathrm{R} \mathbf{Q}
$$

Combining Equations (4.5.26) and (4.5.27) and assuming that $R \neq 0$

$$
\mathbf{Q}: \dot{\xi}=0
$$

or

$$
Q:(\dot{S}-\dot{\alpha})=0
$$

A geometric interpretation of Equation (4.5.29) is shown in Figure 4.5.3, where it can be seen that the backstress moves in a direction parallel to the normal to the yield surface.

We must now decide how $\dot{\alpha}$ is defined. Recall that for the isotropic hardening case, Equation (4.5.29),

$$
\mathrm{Q}: \dot{\sigma}=\sqrt{\frac{2}{3}} \mathrm{H}^{\prime} \overline{\mathrm{d}}^{\mathrm{pl}}=\frac{2}{3} \mathrm{H}^{\prime} \gamma
$$

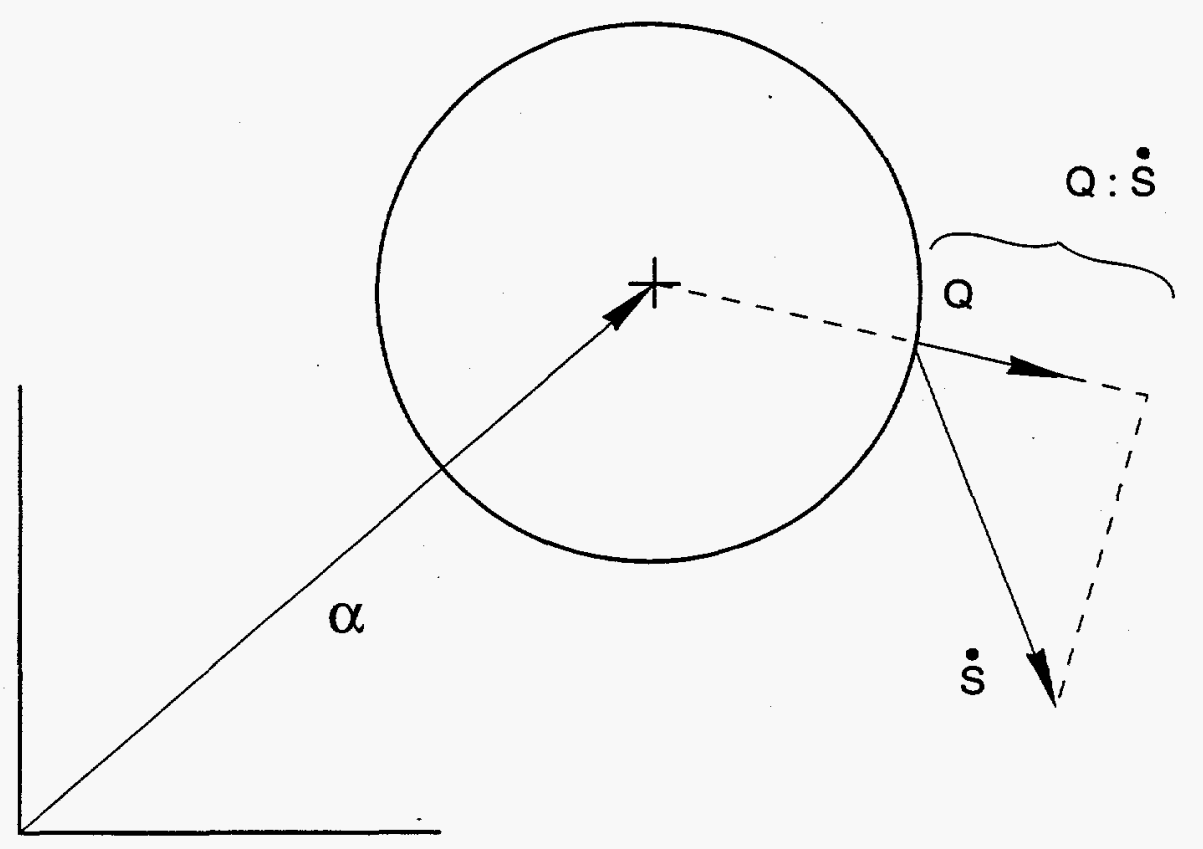

TRI-6348-9-0

Figure 4.5.3. Geometric interpretation of the consistency condition for kinematic hardening. 
The kinematic hardening condition assumes that

$$
\dot{\alpha}=\phi \mathrm{d}^{\mathrm{pl}}=\phi \gamma \mathbf{Q}
$$

where $\phi$ is a material parameter. Equation (4.5.31) combined with Equation (4.5.29) gives a result identical to the isotropic hardening case, Equation (4.5.30), if $\phi$ is chosen to be $\frac{2}{3} H^{\prime}$. Hence, either Equation (4.5.30) or (4.5.31) gives us a scalar condition on $\dot{\alpha}$. Note that both of these are assumptions and must be shown to be reasonable. Of course, experience with material models based on these assumptions has proven them to be reasonable representations of material behavior.

Using Equation (4.5.30), the strain rate decomposition, Equation (4.5.8), and the elastic strain rate, Equation (4.5.12), in the consistency condition for kinematic hardening, Equation (4.5.29) gives

$$
\frac{2}{3} H^{\prime} \gamma \mathbf{Q}=\dot{\sigma}^{\mathrm{tr}}-\mathbf{C}: \mathbf{d}^{\mathrm{pl}}
$$

Taking the tensor inner product of both sides of Equation (4.5.32) with $\mathbf{Q}$ gives

$$
\mathbf{Q}: \frac{2}{3} \mathrm{H}^{\prime} \gamma \mathbf{Q}=\mathbf{Q}:\left(\dot{\boldsymbol{\sigma}}^{\mathrm{tr}}-2 \mu \gamma \mathbf{Q}\right)
$$

Again, because $\mathbf{Q}$ is deviatoric; $\mathbf{C}: \mathbf{Q}=2 \mu \mathrm{Q}$ and $\mathrm{Q}: \mathrm{C}: \mathrm{Q}=2 \mu$.

Solving Equation (4.5.33) for $\gamma$ gives

$$
\gamma=\frac{1}{\left(1+H^{\prime} / 3 \mu\right)} \mathbf{Q}: \mathbf{d}
$$

which is the same result as was obtained for the isotropic hardening case.

\subsubsection{Combined Isotropic and Kinematic Hardening}

For the combined hardening case, we define a scalar parameter, $\beta$, which determines the amount of each type of hardening. We require that

$$
0 \leq \beta \leq 1
$$

Figure 4.5.4 illustrates the uniaxial response which will be computed for $\bar{\sigma}$ for different choices of $\beta$. When $\beta=0$ we have only kinematic hardening, and when $\beta=1$ we have only isotropic hardening. 


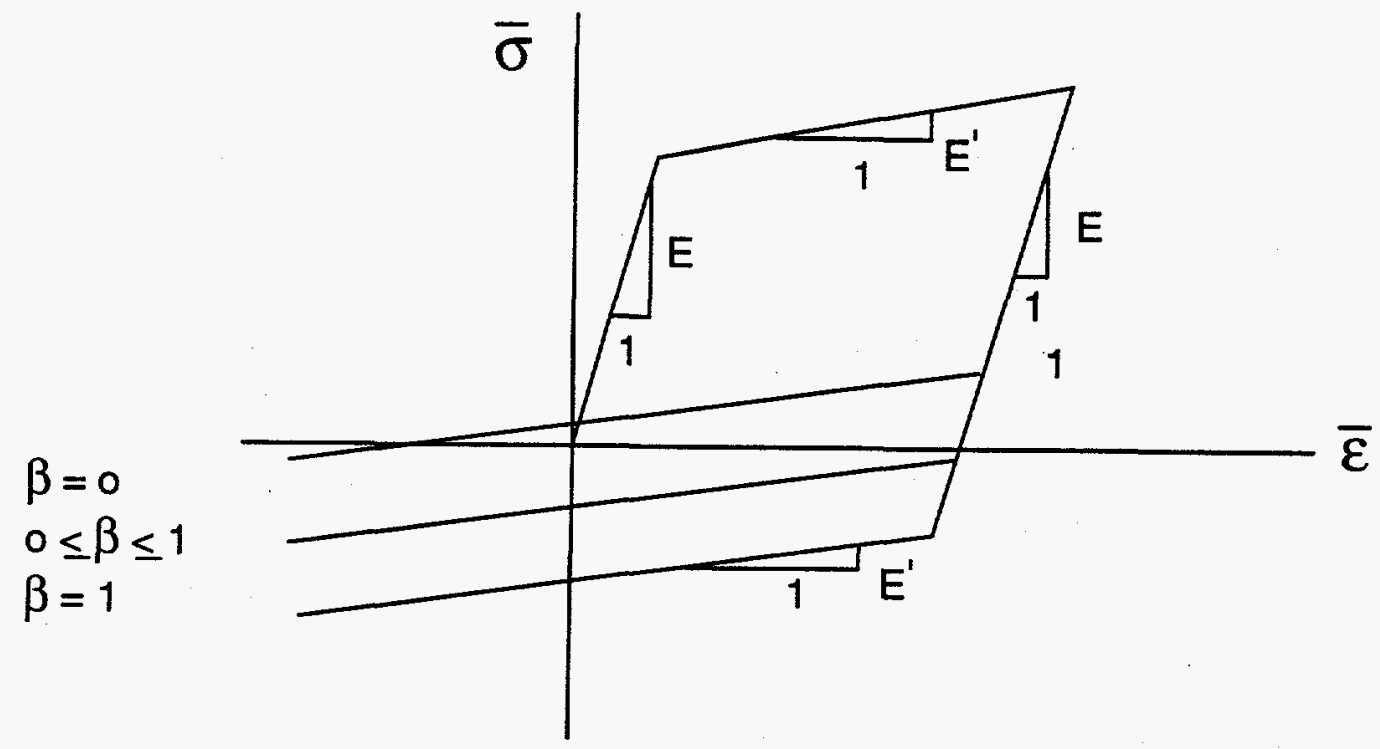

TRI-6348-10-0

Figure 4.5.4. Effect of the choice of the hardening parameter, $\beta$, on the computed uniaxial response.

$$
\dot{\mathrm{R}}=\sqrt{\frac{2}{3}} \mathrm{H}^{\prime} \overline{\mathrm{d}}^{\mathrm{pl}} \beta
$$

and

$$
\dot{\alpha}=\frac{2}{3} \mathrm{H}^{\prime} \mathrm{d}^{\mathrm{pl}}(1-\beta)=\frac{2}{3} \mathrm{H}^{\prime} \mathrm{QQ}(1-\beta) .
$$

As before, we write a consistency condition

$$
\mathbf{Q}: \dot{\xi}=\dot{R}
$$

or

$$
\mathbf{Q}:(\dot{\mathbf{S}}-\dot{\alpha})=\sqrt{\frac{2}{3}} \mathrm{H}^{\prime} \overline{\mathrm{d}}^{\mathrm{pl}} \beta
$$

Using the elastic stress rate and the additive strain rate decomposition with Equation (4.2.56) and taking the tensor product with the normal, $\mathbf{Q}$

$$
\mathbf{Q}: \dot{\sigma}^{\mathrm{rr}}-\gamma \mathbf{Q}: \mathbf{C}: \mathbf{Q}-\mathbf{Q}:\left[\frac{2}{3} \mathrm{H}^{\prime} \gamma(1-\beta)\right] \mathbf{Q}=\mathbf{Q}:\left[\sqrt{\frac{2}{3}} \mathrm{H}^{\prime} \sqrt{\frac{2}{3}} \beta \gamma\right]: \mathbf{Q} \text {. }
$$


Solving for $\gamma$

$$
\gamma=\frac{1}{\left(1+H^{\prime} / 3 \mu\right)} \mathbf{Q}: \mathbf{d}
$$

which is the same result obtained for each of the independent cases.

We summarize the governing equations for the combined theory:

$$
\begin{gathered}
\dot{\sigma}=\mathbf{C}:\left(\mathbf{d}-\mathbf{d}^{\mathrm{pl}}\right)=\dot{\sigma}^{\mathrm{tr}} \\
\dot{\mathrm{R}}=\beta \sqrt{\frac{2}{3}} \mathrm{H}^{\prime} \overline{\mathrm{d}}^{\mathrm{pl}}=\beta \frac{2}{3} \mathrm{H}^{\prime} \gamma \\
\dot{\alpha}=(1-\beta) \frac{2}{3} \mathrm{H}^{\prime} \mathbf{d}^{\mathrm{pl}} \\
\mathbf{d}^{\mathrm{pl}}=\left\{\begin{array}{l}
0, \text { elastic; } \mathrm{f}(\xi)<\kappa^{2} \\
\gamma \mathrm{Q}, \text { plastic; } \mathrm{f}(\xi) \geq \kappa^{2} \\
\mathbf{Q}=\frac{1}{\left(1+\mathrm{H}^{\prime} / 3 \mu\right)} \mathbf{Q}: \mathbf{d} \\
\frac{\partial \mathrm{f}}{\partial \xi} /\left|\frac{\partial \mathrm{f}}{\partial \xi}\right|=\xi / \mathrm{R}
\end{array}\right.
\end{gathered}
$$

\subsubsection{Numerical Implementation}

Our finite element algorithm requires an incremental form of Equations (4.5.41) through (4.5.43). Additionally, we must have an algorithm which integrates the incremental equations subject to the constraint that the stress remains on the yield surface.

The incremental analogs of Equations (4.5.42) through (4.5.44) are

$$
\begin{gathered}
\sigma_{\mathrm{n}+1}=\sigma_{\mathrm{n}+1}^{\mathrm{tr}}-\Delta \gamma 2 \mu \mathrm{Q} \\
\mathrm{R}_{\mathrm{n}+1}=\mathrm{R}_{\mathrm{n}}+\frac{2}{3} \beta H^{\prime} \Delta \gamma
\end{gathered}
$$

and

$$
\alpha_{n+1}=\alpha_{n}+(1-\beta) \frac{2}{3} H^{\prime} \Delta \gamma Q
$$

where $\Delta \gamma$ represents the product of the time increment and the equivalent plastic strain rate $(\Delta \gamma=\Delta \mathrm{t} \gamma)$ 
The subscripts $n$ and $n+1$ refer to the beginning and end of a time step, respectively.

We also need an incremental analog to the rate forms of the consistency condition given by Equations (4.5.13), (4.5.25), and (4.5.39). At the end of the time step, we insist that the stress state must be on the yield surface. Hence, the incremental consistency condition is

$$
\alpha_{n+1}+R_{n+1} Q=S_{n+1}
$$

Equation (4.5.51) is shown graphically in Figure 4.5.5.

Substituting the definitions given by Equations (4.5.48) through (4.5.50) into the consistency condition of Equation (4.5.51)

$$
\left[\alpha_{n}+(1-\beta) \frac{2}{3} H^{\prime} \Delta \gamma \mathbf{Q}\right]+\left[R_{n}+\frac{2}{3} \beta H^{\prime} \Delta \gamma\right] \mathbf{Q}=\left[\mathbf{S}_{n+1}^{\mathrm{tr}}-\Delta \gamma 2 \mu \mathbf{Q}\right] .
$$

Taking the tensor product of both sides of Equation (4.5.52) with $\mathbf{Q}$ and solving for $\Delta \gamma$

$$
\Delta \gamma=\frac{1}{2 \mu} \frac{1}{\left(1+H^{\prime} / 3 \mu\right)}\left(\xi_{n+1}^{\operatorname{tr}} \mid-R_{n}\right)
$$

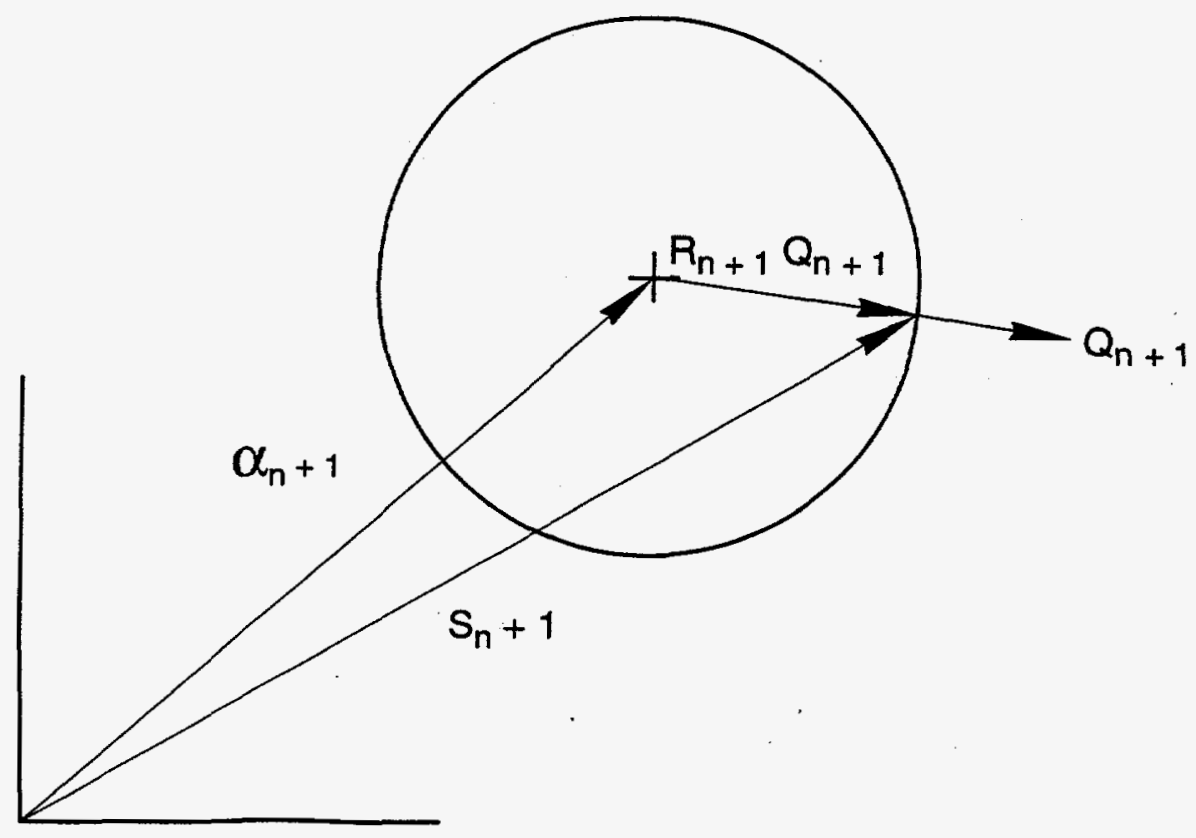

TRI-6348-11-0

Figure 4.5.5. Geometric interpretation of the incremental form of the consistency condition for combined hardening. 
It follows from Equation (4.5.53) that the plastic strain increment is proportional to the magnitude of the excursion of the elastic trial stress past the yield surface (see Figure 4.5.6).

Using the result of Equation (4.5.53) in Equations (4.5.48) through (4.5.50) completes the algorithm. In addition we can compute

$$
\Delta \mathbf{d}^{\mathrm{pl}}=\mathbf{Q} \Delta \gamma
$$

and

$$
\Delta \overline{\mathrm{d}}^{\mathrm{pl}}=\sqrt{\frac{2}{3}} \Delta \gamma
$$

The results of Equation (4.5.53) applied to Equation (4.5.48) show that the final stress is calculated by returning the elastic trial stress radially to the final yield surface at the end of the time step (hence the derivation of the name Radial Return Method). Estimates of the accuracy of this method and other methods for similarly integrating the rate equations are available in Krieg and Krieg (1977) and Schreyer et al. (1979) Note that the last term in Equation (4.5.48) (the radial return correction) is purely deviatoric.

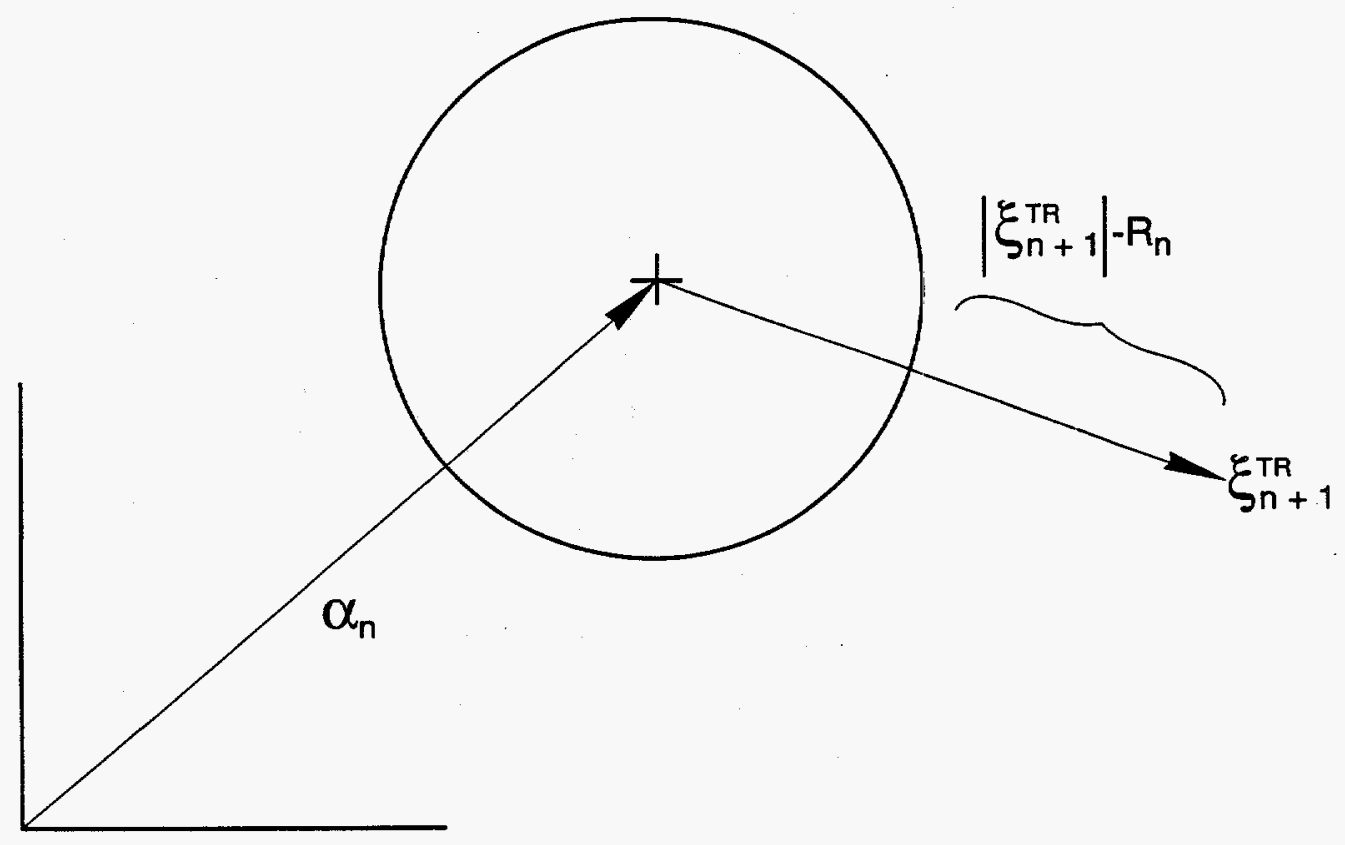

Figure 4.5.6. Geometric interpretation of the radial return correction. 
The elastic plastic material model uses six internal state variables:

EQPS - equivalent plastic strain

RADIUS - current radius of yield surface

ALPHA11 - 1,1 component of backstress in unrotated configuration

ALPHA22 - 2,2 component of backstress in unrotated configuration

ALPHA33 -. 3,3 component of backstress in unrotated configuration

ALPHA12 - 1,2 component of backstress in unrotated configuration.

The PROP array for this material contains the following entries:

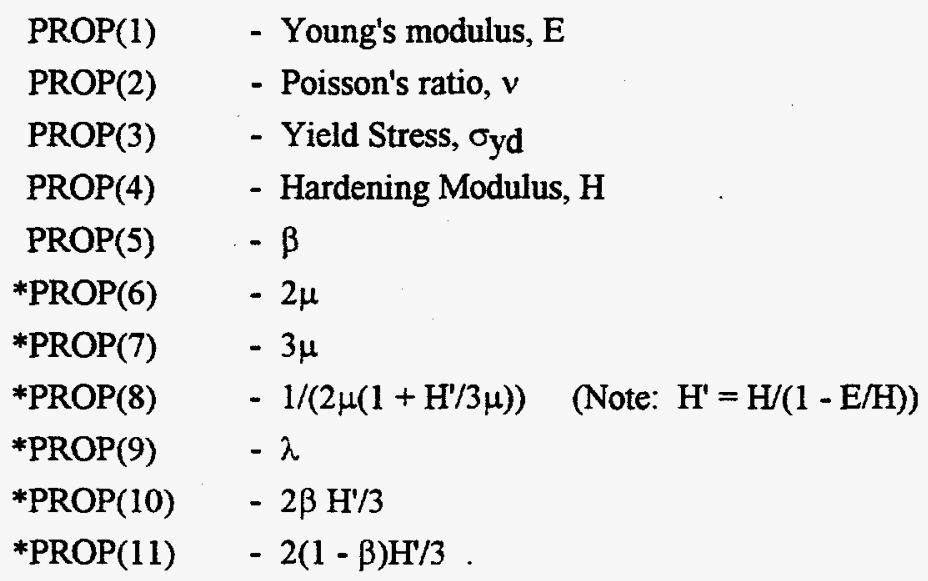

\subsection{Soils and Crushable Foams Model}

The soils and crushable foams model in SANTOS is a direct descendant of the model developed by Krieg (1978). One major difficulty with the original version of this material model which has confounded users is that the pressure dependence of the yield stress is expressed in terms of $\mathrm{J}_{2}$, the second invariant of the stress tensor. We have reformulated the model so that the yield stress is written directly in terms of the pressure. NOTE: this means that old data must be converted.

The yield surface assumed is a surface of revolution about the hydrostat in principal stress space as shown in Figure 4.6.1. In addition, a planar end cap on the normally open end is assumed. The yield stress is specified as a polynomial in pressure, $p$ (positive in compression)

$$
\sigma_{y d}=a_{0}+a_{1} p+a_{2} p^{2}
$$

The determination of the yield stress from Equation (4.3.1) places severe restrictions on the admissible values of $a_{0}, a_{1}$, and $a_{2}$. There are three valid cases as shown in Figure 4.6.2. First, the user may specify a positive $a_{0}$, and $\mathrm{a}_{1}$ and $\mathrm{a}_{2}$ equal to zero as shown in Figure 4.6.2a. This gives an elastic-perfectly plastic deviatoric response, and the yield surface is a cylinder oriented along the hydrostat in principal stress space. Second, a conical yield surface 


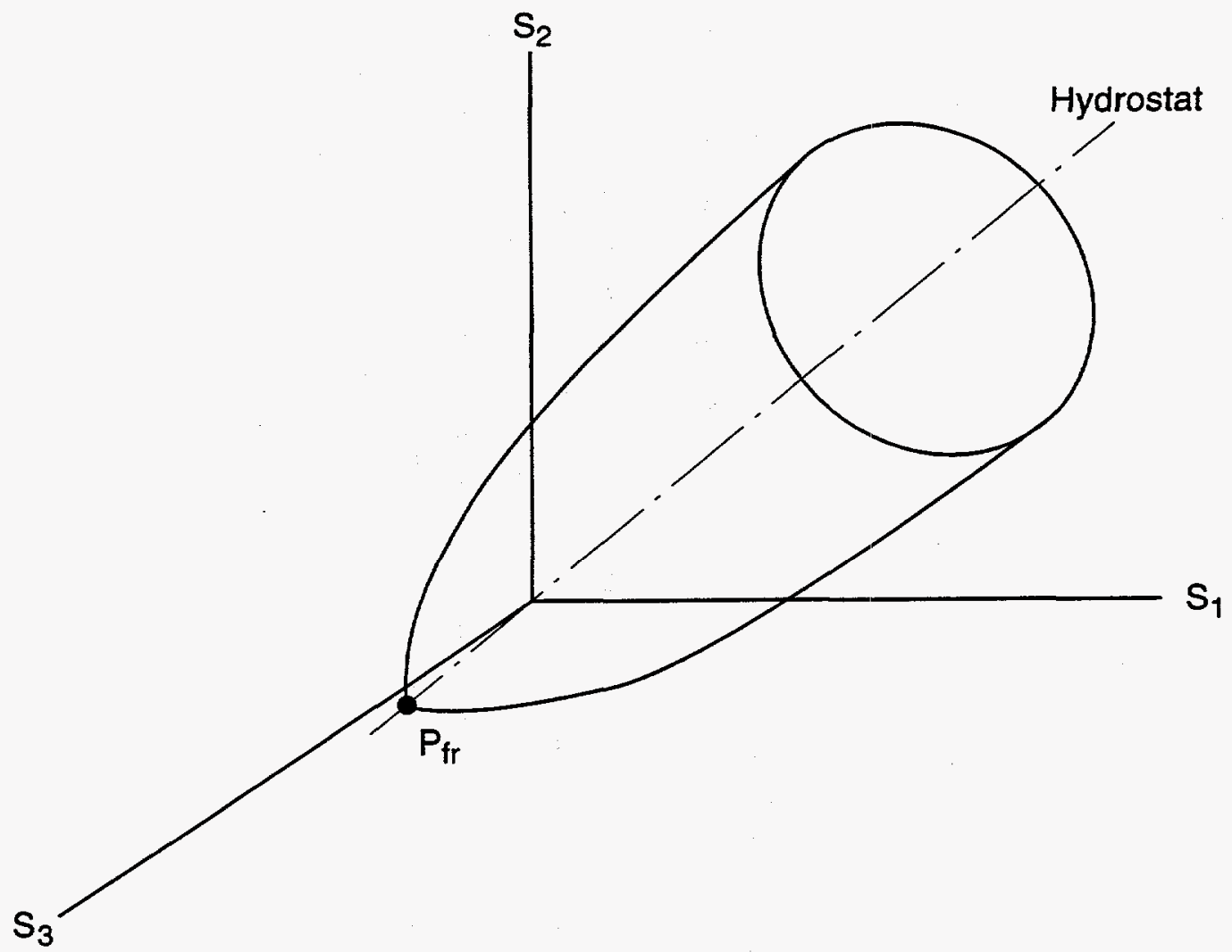

TP1-6348-13-0

Figure 4.6.1. Pressure-dependent yield surface for the soils and crushable foams material model.

(Figure 4.6.2b) is given by setting $a_{2}$ to zero and entering appropriate values of $a_{0}$ and $a_{1}$. The program checks the user's input to determine whether a valid (negative) tensile fracture pressure, $P_{\mathrm{fr}}$, results from the input data. The third case results when all three constants are nonzero and the program detects that a valid negative tensile failure pressure can be derived from the data. This case is shown in Figure 4.6.2c. A valid set of constants for the third case results in a parabola as shown in Figure 4.6.2c. We have drawn the descending portion of the curve with a dashed line, indicating that the program does not use that portion of the curve. Instead, when the pressure exceeds $\mathrm{P*}$, the yield stress is held constant as shown at the maximum value.

The plasticity theories for the volumetric and deviatoric parts of the material response are completely uncoupled. The volumetric response is computed first. The mean pressure, $p$, is assumed to be positive in compression, and a yield function is written for the volumetric response as

$$
\phi_{\mathrm{p}}=\mathrm{p}-\mathrm{f}_{\mathrm{p}}\left(\varepsilon_{\mathrm{v}}\right)
$$

where $f_{p}\left(\varepsilon_{v}\right)$ defines the volumetric stress-strain curve for the pressure as shown in Figure 4.6.3. This function is defined by the user with the restriction that the slope of the function must be less than or equal to the unloading bulk modulus, $\mathrm{K}_{\mathrm{O}}$, everywhere. If the user wishes the volumetric response to be purely elastic, he simply specifies no function identification (e.g., FUNCTION ID $=0$ ). The yield function, $\phi_{\mathrm{p}}$, determines the motion of the end cap along the hydrostat. 


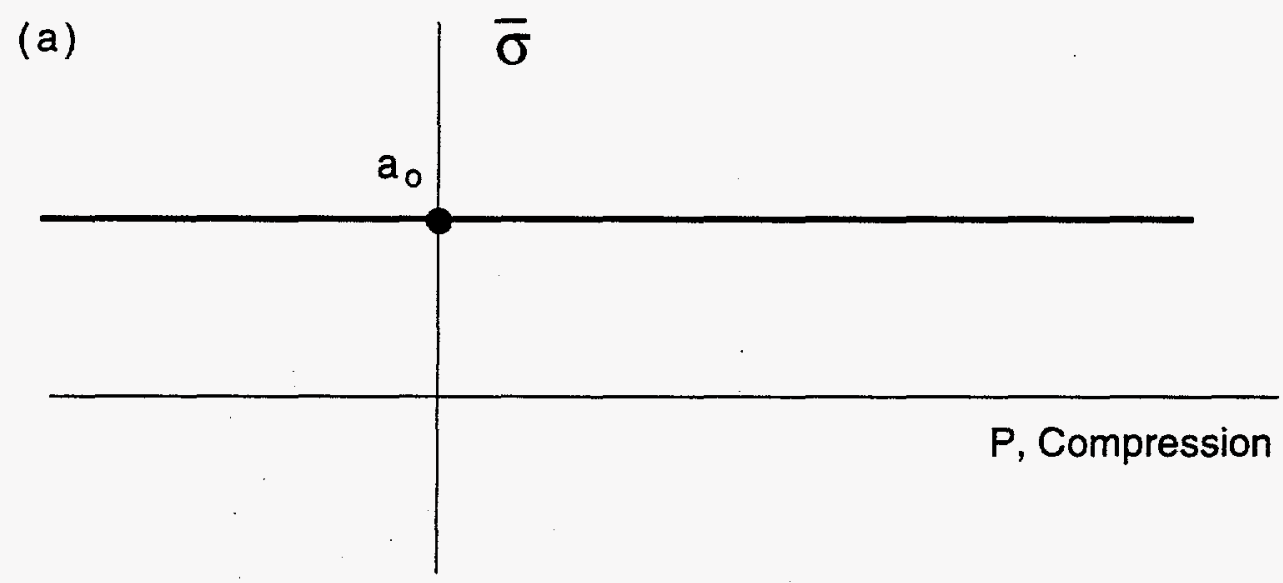

(b)

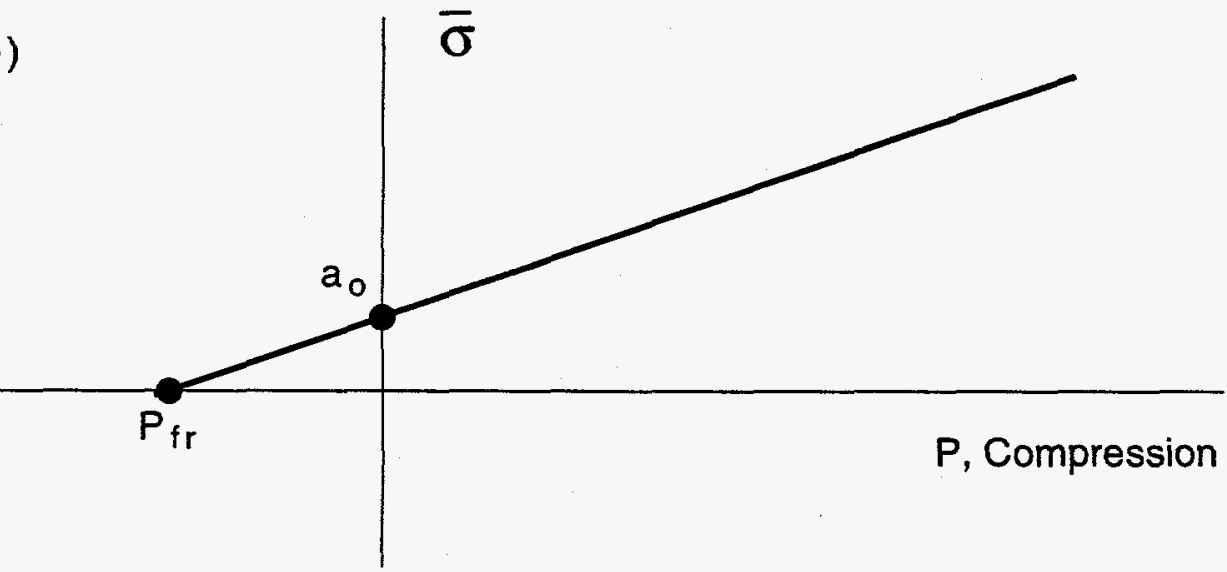

(c)

$\bar{\sigma}$

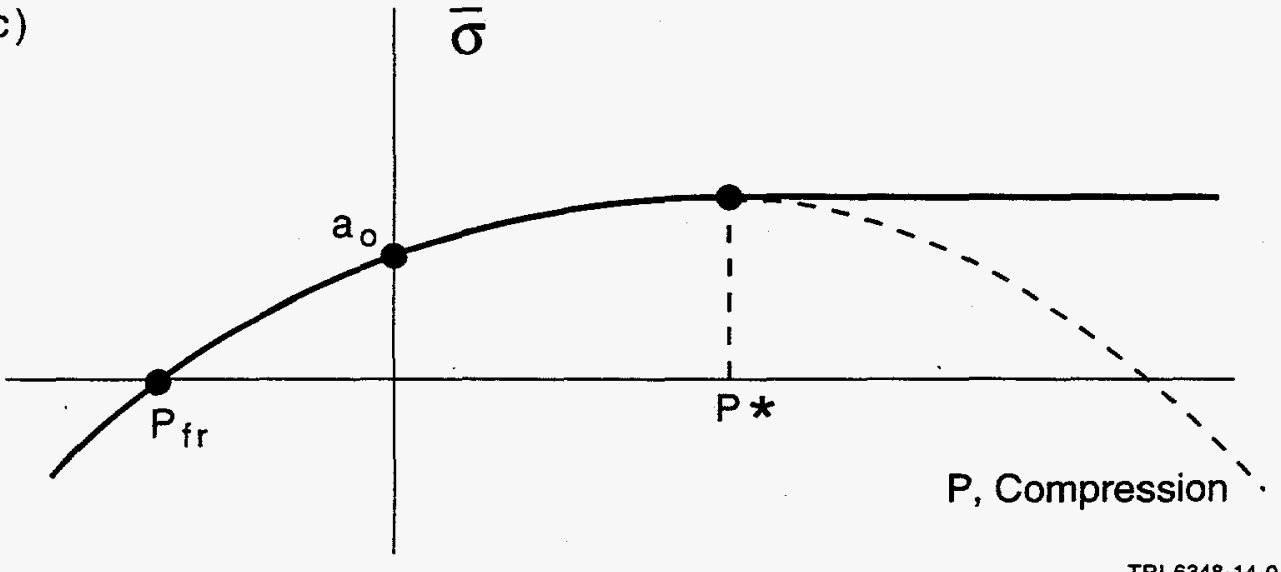

Figure 4.6.2. Forms of valid yield surface which can be defined for the soils and crushable foams material model. 


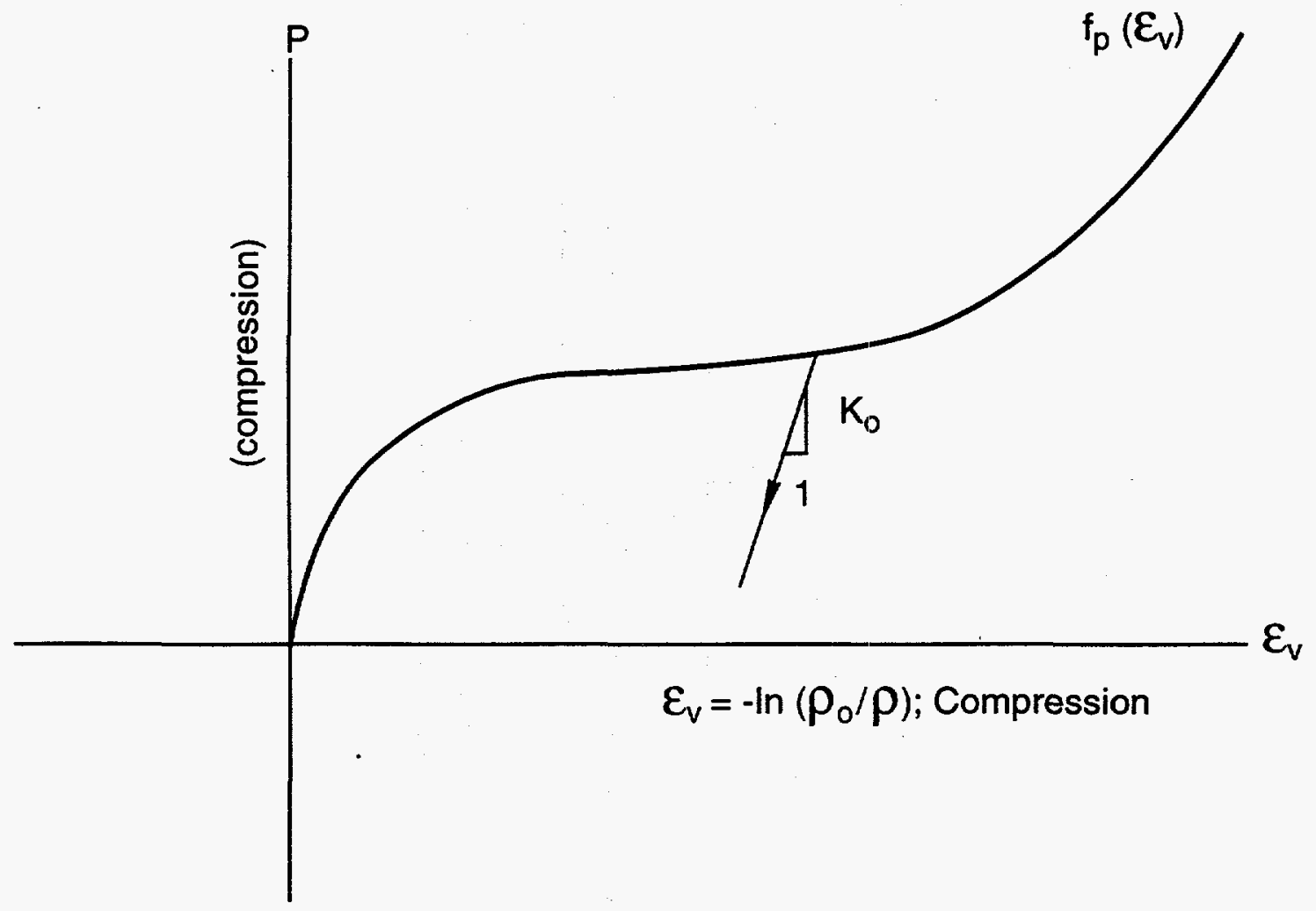

TRI-6348-15-0

Figure 4.6.3. Pressure versus volumetric strain curve in terms of a user-defined curve, $F\left(\varepsilon_{v}\right)$, for the soils and crushable foams material model. 
The mean volumetric strain is updated as

$$
\varepsilon_{\mathrm{v}}^{\mathrm{n}+1}=\varepsilon_{\mathrm{v}}^{\mathrm{n}}+\Delta \mathrm{t} \dot{\varepsilon}_{\mathrm{v}}
$$

where $\dot{\varepsilon}_{\mathrm{V}}$ is the volumetric part of the strain rate $\left(\dot{\varepsilon}_{\mathrm{v}}=\frac{1}{3} \operatorname{tr} \mathbf{d}\right)$.

There are three possible regimes of the pressure-volumetric strain response. Tensile failure is assumed to occur if the pressure becomes smaller (more negative) than $P_{f r}$. The quantity $\varepsilon_{f r}$ is initialized to $-P_{f r} / K_{o}$ by the program. If tensile failure is detected, the pressure is set to $-\mathrm{P}_{\mathrm{fr}}$. Remember, pressure is negative in tension! Failure by monotonic tensile loading is shown in Figure 4.6.4a. As long as $\varepsilon_{\mathrm{v}}<\varepsilon_{\mathrm{fr}}$, the pressure will remain equal to $-\mathrm{P}_{\mathrm{fr}}$.

If the volumetric strain exceeds $\varepsilon_{\mathrm{fr}}$, a check is then made to see if

$$
\varepsilon_{\mathrm{v}}<\varepsilon_{\mathrm{u}}
$$

where $\varepsilon_{\mathrm{u}}$ is the most positive (compressive) volumetric strain previously experienced by the material, set initially to zero by the program. If Equation (4.6.4) is satisfied, the step is elastic and

$$
p^{n+1}=p^{n}-K_{o} \Delta \varepsilon_{v}
$$

This elastic response is shown in Figure 4.6.4b.

If Equation (4.6.4) is not satisfied, the volumetric response is along the curve defined by $f_{p}\left(\varepsilon_{v}\right)$ and

$$
p^{n+1}=f_{p}\left(\varepsilon_{v}^{n+1}\right)
$$

and we set

$$
\varepsilon_{\mathrm{u}}=\varepsilon_{\mathrm{v}}^{\mathrm{n}+1}
$$

This response is shown in Figure 4.6.4c. Note, that if Equation (4.6.5) is used to determine p, we also drag $\varepsilon_{\mathrm{fr}}$ along so that if we unload from the curve, $f_{p}\left(\varepsilon_{v}\right)$, we will fracture at the appropriate strain level as shown in Figure 4.6.4d.

The deviatoric part of the response is computed next and uses a conventional plasticity theory with radial return. See Krieg and Krieg (1977). The trial elastic deviatoric stresses are computed as

$$
S^{t r}=S_{n}+2 \mu \Delta t \dot{e}
$$

where $\dot{\mathrm{e}}$ is the deviatoric part of the strain rate. The current value of yield stress is calculated using Equation (4.6.1), and the von Mises effective stress, $\bar{\sigma}$, is computed as 


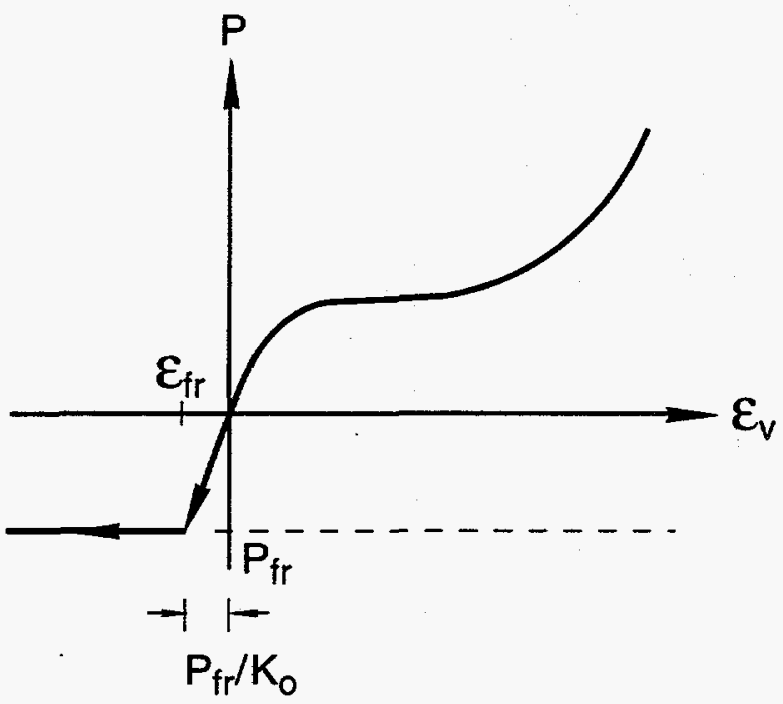

(a)

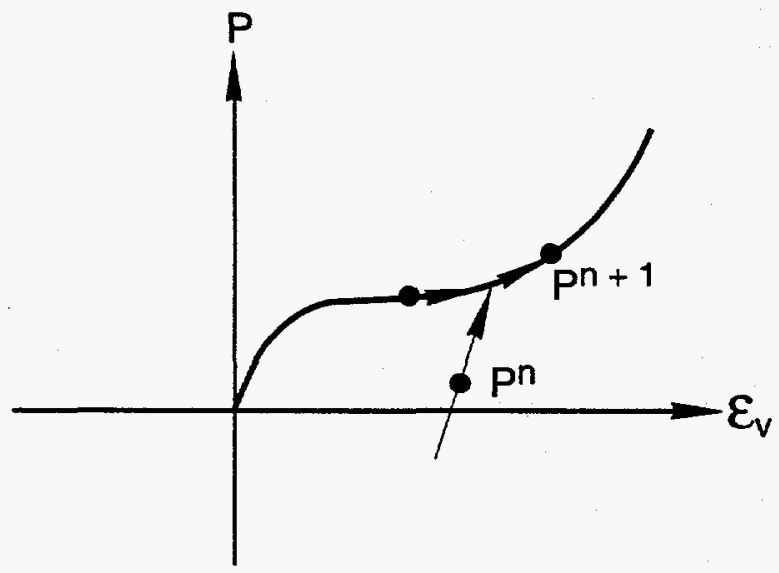

(c)

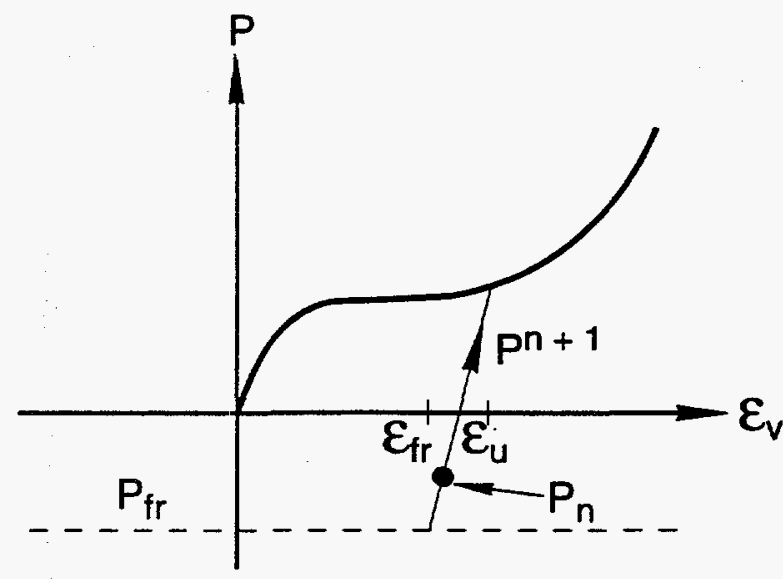

(b)

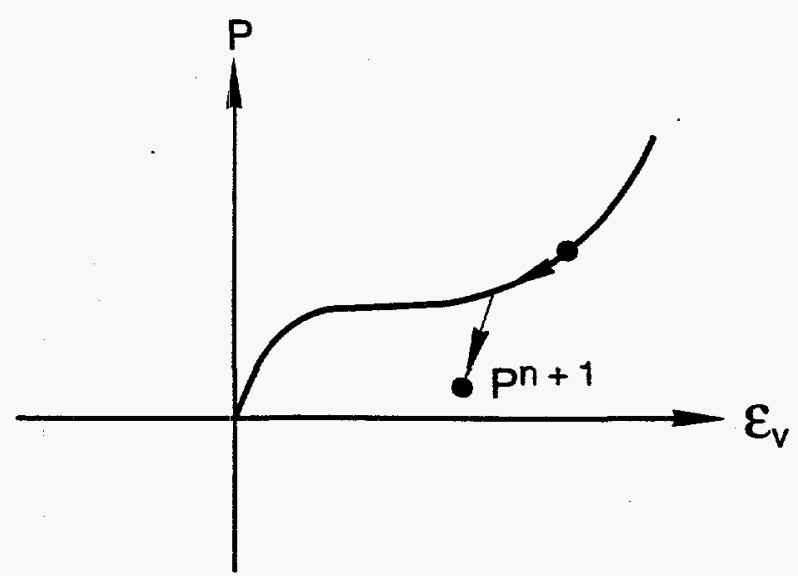

(d)

TRI-6348-16-0

Figure 4.6.4. Possible loading cases for the pressure versus volumetric strain response using the soils and crushable foams material mode. 


$$
\bar{\sigma}=\sqrt{\frac{3}{2} S: S}
$$

The yield condition is checked to determine whether $\bar{\sigma}<\sigma_{\mathrm{yd}}$. If this is the case, the trial stress is the correct deviatoric stress at the end of the time step, $S_{n+1}=S^{\text {tr }}$. If yield is exceeded, a simple radial return is performed to calculate the deviatoric stress at the end of the time step

$$
S_{n+1}=\frac{\sigma_{y d}}{\bar{\sigma}} S^{t r}
$$

Finally, the total stress is determined by

$$
\sigma_{n+1}=S^{n+1}+p^{n+1} \delta
$$

The Soils and Crushable Foams model uses four internal state variables:

$$
\begin{aligned}
& \text { EVMAX - maximum compressive volumetric strain experienced (always positive), } \\
& \text { EVFRAC - current value of volumetric fracture strain (positive in compression), } \\
& \text { EV - current value of volumetric strain (positive in compression), } \\
& \text { NUM - integer pointing to the last increment in the pressure function where the interpolate was }
\end{aligned}
$$

The PROP array contains the following entries for this material:

$$
\begin{array}{ll}
\text { PROP(1) } & -2 \mu \\
\text { PROP(2) } & - \text { Bulk Modulus, } K \\
\text { PROP(3) } & -a_{0} \\
\text { PROP(4) } & -a_{1} \\
\text { PROP(5) } & -a_{2} \\
\text { PROP(6) } & - \text { Function ID number. }
\end{array}
$$

\subsection{Low Density Foams}

The low density foams model presented here was developed by Neilsen, Morgan, and Krieg (1987) and is based on results from experimental tests on low density, closed-cell polyurethane foams. These foams having densities ranging from 2 to 10 pounds per cubic foot have been proposed for use as energy absorbers in nuclear waste shipping containers. Representative responses of closed-cell polyurethane foams for various hydrostatic, uniaxial, and triaxial laboratory test conditions are shown in Figures 4.7.1 and 4.7.2. These results indicate that the volumetric response of the foam is highly dependent on load history. This implies that typical decompositions of total foam response into an independent volumetric part and a mean stress (pressure) dependent deviatoric part are not valid for this class of foam. Many "soil and crushable foam" models, including the other foam model described 


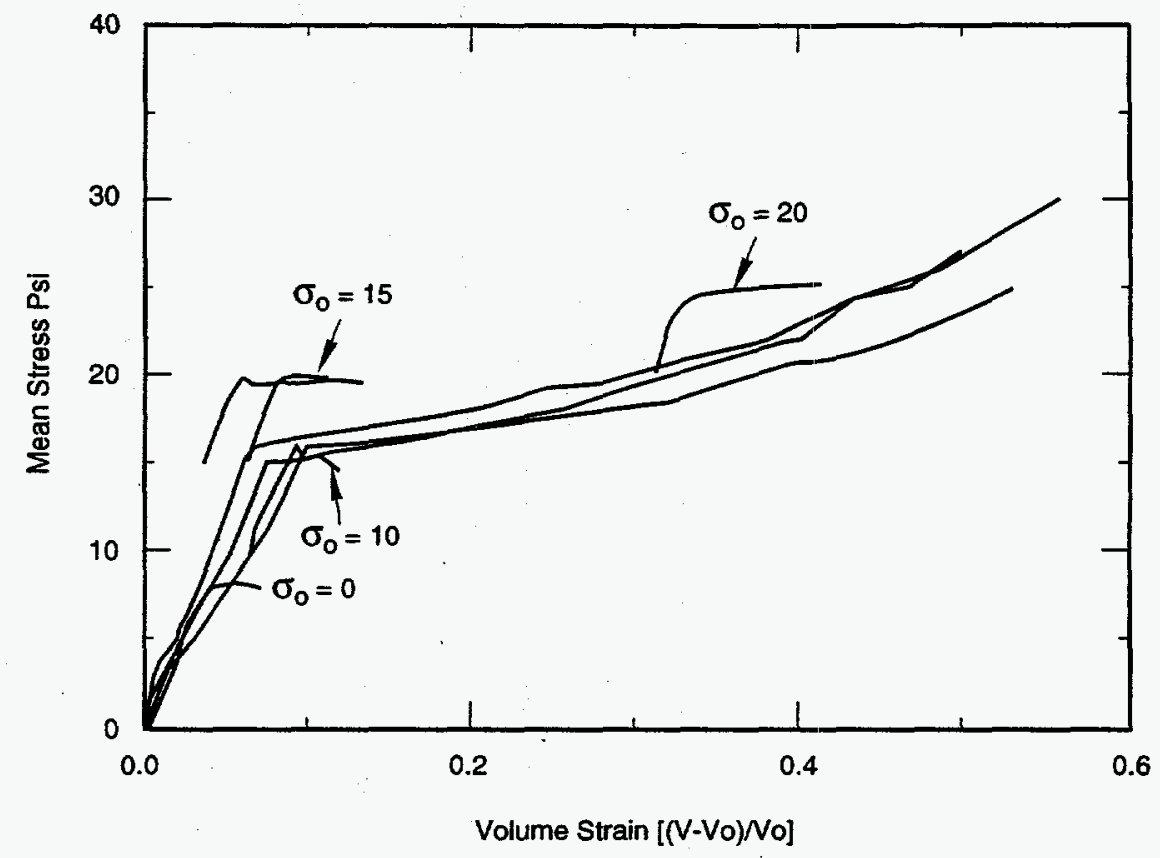

TRI-6348-18-0

Figure 4.7.1. Foam volume strain versus mean stress for 6602 foam at various confining pressures.

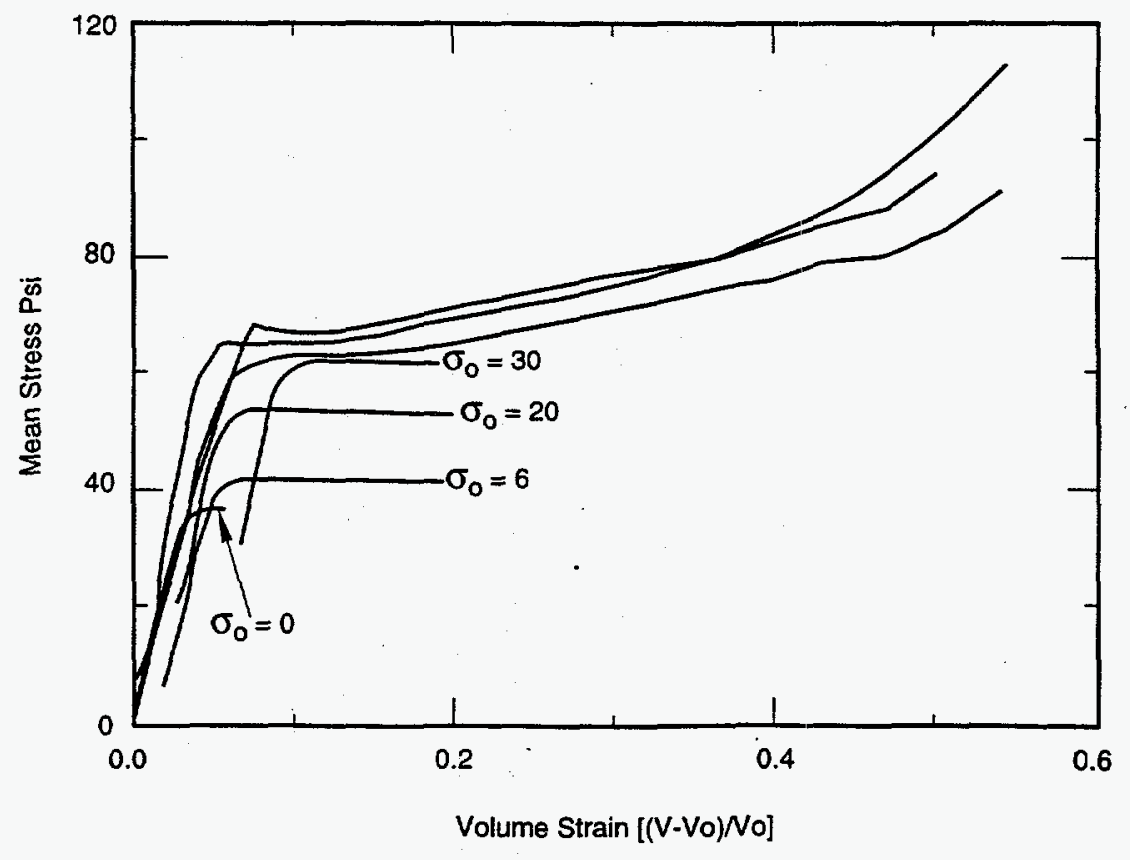

TRI-6348-17-0

Figure 4.7.2. Foam volume strain versus mean stress for 9505 foam at various confining pressures. 
in Section 4.6, use such decompositions and hence are not valid for low-density, closed-cell polyurethane foams. The model presented here reproduces experimental test responses more accurately for this class of foams than the model in Section 4.6.

The experimental tests on which this model is based were performed by the Civil Engineering Research Facility of the University of New Mexico with the results reported in (Neilsen et al., 1987). Foam samples were subjected to static, compressive stresses during these tests. In most of the tests, air was trapped in the closed cells of the foams and could not escape because the samples were jacketed with an impervious material. In this constitutive model, the total foam response is decomposed into contributions from the skeleton and from air trapped in the closed cells of the foam. The contribution of the air to the total foam response is dependent on the application. If the foam is used in a vented application where the air can escape, the contribution of the air is zero and the foam and skeleton responses are identical. If the foam is used in an application where the air cannot escape (such as a sealed shipping container) the foam pressure is considered to be the sum of pressure carried by the skeleton and the air pressure. That is,

$$
\mathrm{p}_{\mathrm{f}}=\mathrm{p}_{\mathrm{sk}}+\mathrm{p}_{\mathrm{air}}
$$

where $\mathrm{p}_{\mathrm{f}}$ and $\mathrm{p}_{\mathrm{sk}}$ are the mean stresses (first invariants of the stress tensor divided by three) of the foam and skeleton, respectively. The mean stresses and air pressure are assumed positive in tension. The air pressure is determined from

$$
\mathrm{p}_{\mathrm{air}}=\frac{\mathrm{p}_{\mathrm{o}} \gamma}{1+\gamma-\phi}
$$

where $\gamma$ is the engineering volume strain (first invariant of the total strains), which is positive in tension and $p_{0}$ and $\phi$ are model parameters. The parameter $\mathrm{p}_{\mathrm{o}}$ is the initial foam pressure (usually atmospheric pressure of $14.7 \mathrm{psi}$ ), and $\phi$ is the ratio of the foam density to the polymer density from which the foam is produced.

Test data indicate that the skeleton response in any principal stress direction is independent of loading in any other principal stress direction. Thus, Poisson's ratio for the skeleton is equal to zero. Test data also indicate that the yield strength of the skeleton in any principal stress direction can be expressed in terms of the engineering volume strain and the second invariant of the deviatoric strains with the following relationship

$$
f_{i}=\left\{\begin{array}{l}
A+B(1+C \gamma) ; \Pi_{\varepsilon}^{\prime}>0 \\
B(1+C \gamma) ; \Pi_{\varepsilon}^{\prime}=0
\end{array}\right.
$$

where $\Pi_{\varepsilon}^{\prime}$ is the second invariant of the deviatoric strain tensor; $\gamma$ is the engineering volume strain as in Equation (4.7.2); A, B, and C are constants determined from fitting Equation (4.7.3) to the laboratory data. Constants B and C are determined from hydrostatic test data where $\Pi_{\varepsilon}^{\prime}$ is zero, and $A$ is determined from any test where the loading is deviatoric.

Numerical implementation of the model is as follows. Foam stresses and strains from the previous time increment are saved. At the beginning of the next time increment, the old skeleton stresses are computed from the old foam 
stresses and the old air pressure. The strain rates for the new time increment are used to determine new strain increments and trial elastic stress increments for the skeleton. These stress increments are added to the old skeleton stresses to produce new trial stresses for the skeleton. The trial skeleton stresses are then rotated to principal stress directions and compared with the yield stress determined from Equation (4.7.3). If yield occurs, the skeleton stresses are set to the yield stress. If yield does not occur, the trial skeleton principal stresses become the final skeleton principal stresses. The final skeleton stresses are obtained by rotating the final skeleton principal stresses back to the unrotated configuration. .Then, the final foam stresses are obtained by adding the air pressure contribution for the new strain state to the new skeleton stresses.

Input parameters for the model are the constants $\mathrm{E}, \mathrm{P}_{\mathrm{O}}, \phi, \mathrm{A}, \mathrm{B}$, and $\mathrm{C}$, which are defined above. If the foam is used in an application where the air can escape, $p_{0}$ should be input as zero. Otherwise, $p_{0}$ is the atmospheric pressure at the beginning of the simulation.

There are no internal state variables for this model.

The PROP array contains the following entries for this material type:

$\begin{array}{ll}\text { PROP(1) } & - \text { Young's modulus, E } \\ \text { PROP(2) } & - \text { A } \\ \text { PROP(3) } & - \text { B } \\ \text { PROP(4) } & - \text { C } \\ \text { PROP(5) } & - \text { NAIR } \\ \text { PROP(6) } & -P_{0} \\ \text { PROP(7) } & -\phi .\end{array}$

\subsection{Elastic-Plastic Power Law Hardening Material}

One of the more commonly used models in the SANTOS material library is the elastic-plastic combined isotropic/kinematic hardening model. This model considers the hardening modulus to be a constant, which means that the post-yield effective stress, $\bar{\sigma}$, versus effective plastic strain, $\bar{\varepsilon}_{\mathrm{p}}$, relationship is linear. For a large class of important problems, a linear $\bar{\sigma}$ versus $\bar{\varepsilon}_{\mathrm{p}}$ relationship may be adequate for the post-yield behavior over the range of interest. This, however, places a severe restriction on the materials to be modeled or requires a priori information about the expected strain levels in the problem so that an approximate hardening modulus may be selected to produce a good approximation to the correct stress state based on the expected strain values. In addition, the strain range of interest must be small (no large strain gradients) so that the linear hardening relationship is applicable. However, there are classes of problems in which the linear approximation for plastic hardening is inadequate. A constant hardening modulus cannot adequately describe the post-yield behavior to predict structural behavior in the detail required. Determination of limit load response is an example of a class of problems for which linear hardening is inappropriate.

To overcome these restrictions, a variable hardening plasticity model (Stone et al., 1990) has been included in SANTOS. The use of piecewise linear segments to represent the hardening curve was an initial consideration based on the capability to match any material hardening behavior, but the resulting material model subroutine was not 
not amenable to vectorization. Vectorization requirements limit the form of the model to a functional relationship between effective stress and effective plastic strain. The form of the current implementation considers the postyield stress to be described by a power law involving the equivalent plastic strain with the option to include a Lüders strain segment. The form of the hardening model was selected for its simplicity and ability to match the post-yield behavior of many engineering materials. The model has the form during a plastic loading process

$$
\bar{\sigma}-\sigma_{\mathrm{ys}}=\mathrm{A}\left\langle\bar{\varepsilon}_{\mathrm{p}}-\varepsilon_{\mathrm{L}}\right\rangle^{\mathbf{m}}
$$

where $\mathrm{A}$ and $\mathrm{m}$ are material constants, $\bar{\varepsilon}_{\mathrm{p}}$ is the equivalent plastic strain, $\bar{\sigma}$ is the effective stress, $\sigma_{\mathrm{ys}}$ is the initial yield stress, and $\varepsilon_{L}$ is the Lüders strain or yield plateau strain. The use of brackets in the above equation denotes the use of a Heaviside function. The function is zero until the arithmetic expression within the brackets becomes positive. The material constants for this model can be determined from the measured stress versus strain data through simple curve fitting techniques. By suitably choosing the material constants $\mathrm{A}$ and $\mathrm{m}$, the form of the model can represent either elastic/perfectly plastic or linear hardening material behavior in addition to the power law hardening response. The proposed material model is strictly valid for isotropic hardening behavior where the radius of the yield surface grows equally in all directions due to plastic straining.

Many engineering materials exhibit the phenomenon of Lüders straining. A typical stress versus strain curve for such a material is shown in Figure 4.8.1. In reality, Lüders strain does not occur at constant stress but rather in a serrated fashion. Each serration corresponds to the formation of a new Lüders band. The serrations are small enough that a constant stress representation is adequate. In common ferrous alloys, Lüders strain as well as other yield point phenomena are generally associated with the interaction between solute atoms and dislocations.

The constitutive routine is entered with the stress state at the beginning of the step, $S_{\mathrm{ij}}^{\mathrm{n}}$, the strain-rate over the step, $\dot{\varepsilon}_{\mathrm{ij}}$, and the time step, $\Delta \mathrm{t}$. An elastic trial stress, $\mathrm{S}_{\mathrm{ij}}^{\mathrm{tr}}$, is computed and a von Mises yield criterion is used to compute a trial effective stress, $\bar{\sigma}^{\mathrm{tr}}$, which is compared to the current radius of the yield surface. If the trial effective stress is less than the radius of the yield surface, then the load step is elastic and the trial stress becomes the final stress, $S_{\mathrm{ij}}^{\mathrm{n}+1}$. If the effective stress is greater than the radius of the yield surface, then plastic straining will occur over the step and the final stress state and plastic strain increment remain to be computed.

The expression for the stress at the end of the step is

$$
S_{i j}^{n+1}=S_{i j}^{n}+\dot{S}_{i j}\left(S_{i j}^{n+1}\right) \Delta t
$$

where we have used a backward Euler integration for the stress. The stress rate at the end of the step, $\dot{\mathrm{S}}_{\mathrm{ij}}^{\mathrm{n}+1}$, is computed as follows

$$
\dot{\mathrm{S}}_{\mathrm{ij}}^{\mathrm{n}+1}=2 \mu\left(\dot{\varepsilon}_{\mathrm{ij}}-\dot{\varepsilon}_{\mathrm{p}_{\mathrm{ij}}}\right)
$$

where $\dot{\varepsilon}_{\mathrm{p}_{\mathrm{ij}}}$ are the components of plastic strain. 


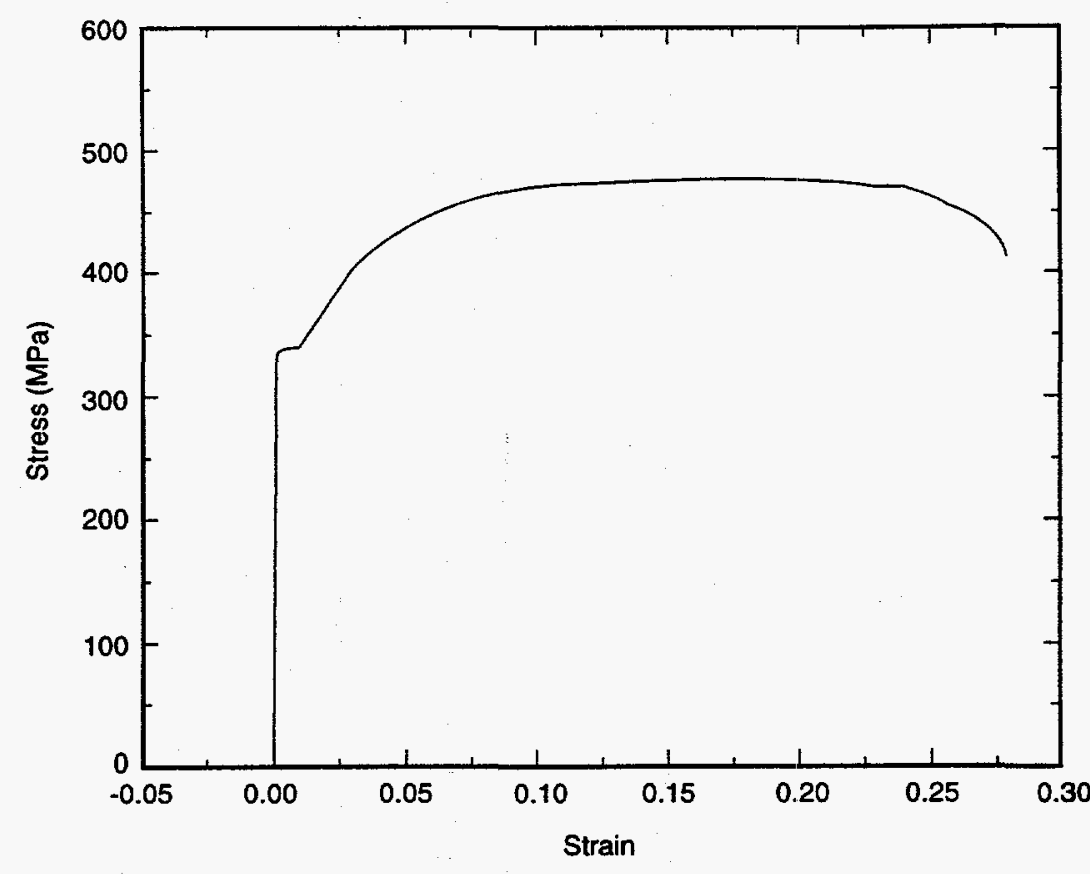

TRI-6348-19-0

Figure 4.8.1. Stress versus strain curve for a typical ferritic steel exhibiting Luiders strain.

For von Mises flow, we can write the above as

$$
\dot{S}_{i j}^{n+1}=2 \mu \dot{\varepsilon}_{i j}-2 \mu\left(\frac{3 \gamma}{2} \frac{S_{i j}^{n+1}}{\bar{\sigma}^{n+1}}\right)
$$

where $\gamma$ is a scalar quantity.

Substituting the above expression for $\dot{S}_{i j}^{n+1}$ into the expression for $S_{i j}^{n+1}$ results in

$$
S_{i j}^{n+1}=S_{i j}^{n}+2 \mu \dot{\varepsilon}_{i j} \Delta t-3 \mu \frac{S_{i j}^{n+1}}{\bar{\sigma}^{n+1}} \Delta \gamma
$$

where $\Delta \gamma=\gamma \Delta \mathrm{t}$. The first two expressions on the right-hand-side of the equation define the trial stress $S_{i j}^{\text {tr }}$ so that the final expression becomes

$$
S_{i j}^{n+1}=S_{i j}^{t r}-\left(3 \mu \frac{S_{i j}^{n+1}}{\bar{\sigma}^{n+1}} \Delta \gamma\right) .
$$


We make use of the following identity

$$
\frac{S_{i j}^{n+1}}{\bar{\sigma}^{n+1}}=\frac{S_{i j}^{t r}}{\bar{\sigma}^{t r}}
$$

to get the final expression for the stress at the end of the step

$$
S_{i j}^{n+1}=S_{i j}^{t r}\left(1-\frac{3 \mu \Delta \gamma}{\bar{\sigma}^{t r}}\right)
$$

The plastic strain increment $\Delta \gamma$, is the only unknown in the equation. To solve for the plastic strain increment, we must go back to our expression for the yield function. Combining the yield function with the expression for $S_{i j}^{n+1}$ and making use of the identity, we get

$$
\bar{\sigma}^{\operatorname{tr}}-3 \mu \Delta \gamma=\sigma_{\mathrm{ys}}+\mathrm{A}\left\langle\bar{\varepsilon}_{\mathrm{p}}^{\mathrm{n}}+\Delta \gamma-\varepsilon_{\mathrm{L}}\right\rangle^{\mathrm{m}}
$$

which is solved for $\Delta \gamma$ using Newton Raphson iteration. The computed value of $\Delta \gamma$ is substituted back into the expression for $S_{i j}^{n+1}$, Equation (4.8.8).

The bulk response is assumed to be linear elastic. The pressure at the end of the step is calculated using the volumetric strain rate, $d_{k k}$, through the relation

$$
\mathrm{p}^{\mathrm{n}+1}=\mathrm{p}^{\mathrm{n}}+\mathrm{Kd}_{\mathrm{kk}} \Delta \mathrm{t}
$$

where $\mathrm{K}$ is the bulk modulus of the material, $\Delta t$ is the time step size, and $\mathrm{p}^{\mathrm{n}}$ is the pressure at the beginning of the step. The stress from the deviatoric and bulk responses are combined to give the final stress state.

The power law hardening material uses two internal state variables:

$$
\begin{array}{ll}
\text { RADIUS } & \text { - current radius of the yield surface } \\
\text { EQPS } & \text { - equivalent plastic strain. }
\end{array}
$$

The PROP array for this material contains the following entries:

$$
\begin{aligned}
& \text { PROP(1) - Young's modulus, E } \\
& \text { PROP(2) - Poisson's ratio, } v \\
& \text { PROP(3) - Yield Stress, } \sigma_{y s} \\
& \text { PROP(4) - Hardening constant, A } \\
& \text { PROP(5) - Hardening exponent, } m \\
& \operatorname{PROP}(6) \quad-\text { Lüders strain, } \varepsilon_{\mathrm{L}} \\
& \text { * PROP(7) - } 2 \mu \\
& \text { * PROP(8) - } 3 \mu \\
& \text { * PROP(9) }-\lambda \text {. }
\end{aligned}
$$




\subsection{Power Law Creep Material Model}

The power law creep material model described here is commonly used to model the time-dependent behavior of metals, brazes, or solders at high homologous temperatures as well as the time-dependent behavior of geologic materials such as salt. The model is cast as a conventional power law secondary creep model of the form

$$
\dot{\varepsilon}_{\mathrm{c}}=\mathrm{A} \bar{\sigma}^{\mathrm{m}} \exp \left(\frac{-\mathrm{Q}}{\mathrm{R} \Theta}\right)
$$

where $\bar{\sigma}$ is the effective deviatoric stress, $A$ and $m$ are material constants, $\Theta$ is the absolute temperature, $R$ is the universal gas constant $\left(1.987 \frac{\mathrm{cal}}{\mathrm{moleK}}\right)$, and $\mathrm{Q}$ is the activation energy.

We choose to write the expression for the deviatoric stress at the end of the step as

$$
S_{i j}^{n+1}=S_{i j}^{n}+\dot{S}_{i j}\left(S_{i j}^{n}\right) \Delta t
$$

where we have used a forward Euler integration for the stress. We can write the stress rate at step $\mathrm{n}$ as

$$
\dot{S}_{\mathrm{ij}}=2 \mu\left(\dot{\varepsilon}_{\mathrm{ij}}-\dot{\varepsilon}_{\mathrm{c}_{\mathrm{ij}}}\right)
$$

where $\dot{\varepsilon}_{\mathrm{c}_{\mathrm{ij}}}$ are the creep strain rate components. The creep strain rate components for von Mises flow are

$$
\dot{\varepsilon}_{\mathrm{c}_{\mathrm{ij}}}=\frac{3}{2} \dot{\bar{\varepsilon}}_{\mathrm{c}} \frac{\mathrm{S}_{\mathrm{ij}}}{\bar{\sigma}}
$$

where $\dot{\bar{\varepsilon}}_{\mathrm{c}}$ is the effective or equivalent creep strain rate. Substituting into the expression for the stress rate gives

$$
\dot{S}_{\mathrm{ij}}^{\mathrm{n}}=2 \mu\left(\dot{\varepsilon}_{\mathrm{ij}}-\frac{3}{2} \mathrm{~A} \exp \left(\frac{-\mathrm{Q}}{\mathrm{R} \Theta}\right) \bar{\sigma}^{\mathrm{m}-1} \mathrm{~S}_{\mathrm{ij}}^{\mathrm{n}}\right)
$$

where $\bar{\sigma}$ is evaluated at step $n$. The stress rate is computed and stored as a state variable for use during step $n+1$.

Numerical analysis of the forward Euler operator shows that the method is conditionally stable. It is possible to calculate an estimate of the critical time step for stability of the forward Euler operator based on the form of the flow potential and the elastic constants (Cormeau, 1975). Following procedures outlined in Cormeau (1975), the critical time step for stability is calculated to be

$$
\Delta \mathrm{t}_{\mathrm{st}}<\frac{4(1+\mathrm{v})}{3 \mathrm{EA} \exp \left(\frac{-\mathrm{Q}}{\mathrm{R} \Theta}\right) \mathrm{m} \bar{\sigma}^{\mathrm{m}-1}}
$$

where $E$ and $v$ are Young's modulus and Poisson's ratio, respectively. Accuracy of the method is assured if the time steps are sufficiently small, but the stable time step does not guarantee an accurate solution. However, our experience with the method has shown that the solution obtained using $\Delta t_{s t}$ is indeed accurate. The standard power 
law secondary creep model is requested in SANTOS by using the material name POWER LAW CREEP while the adaptive time-stepping version is requested by using the name ADAPTIVE PL CREEP.

The volumetric behavior of the material is assumed to be linearly elastic as shown below

$$
\mathrm{p}^{\mathrm{n}+1}=\mathrm{p}^{\mathrm{n}}+\mathrm{Kd}_{\mathrm{kk}} \Delta \mathrm{t}
$$

where $K$ is the bulk modulus, $d_{k k}$ is the volumetric strain rate, $\Delta t$ is the time step size, and $p^{n}$ is(4.9.6)

the pressure at the beginning of the step. The stress from the deviatoric and bulk responses is combined to give the final stress state for the material.

The power law creep material uses a single state variable:

EQCS - equivalent creep strain.

The PROP array for this material contains the following entries:

$$
\begin{array}{ll}
\text { PROP(1) } & -2 \mu \\
\text { PROP(2) } & -\mathrm{K} \\
\text { PROP(3) } & -\mathrm{A} \\
\text { PROP(4) } & -\mathrm{m} \\
\text { PROP(5) } & -\frac{\mathrm{Q}}{\mathrm{R} \Theta} \text { if isothermal or } \frac{\mathrm{Q}}{\mathrm{R}} \text { if not. }
\end{array}
$$

\subsection{Thermoelastic Material Model}

This material model represents the behavior of an elastic material with temperature-dependent material constants. Both Young's modulus and Poisson's ratio are allowed to vary with temperature. The values of Young's modulus and Poisson's ratio at the beginning (step $n$ ) and end (step $n+1$ ) of the time step are stored as state variables. The relationship between the property value and temperature is specified using a FUNCTION definition.

We will choose to separate the stress and strain behavior into bulk and deviatoric response. The resulting equation for the elastic deviatoric stress response is

$$
\mathrm{S}_{\mathrm{ij}}^{\mathrm{n}+1}=2 \mu\left(\dot{\Theta}^{\mathrm{n}+1}\right) \varepsilon_{\mathrm{ij}}^{\mathrm{n}+1}
$$

where the shear modulus $\mu$ is a function of the temperature, $\Theta^{n+1}$, at the end of the step. For our numerical implementation, we will cast the problem in an incremental form. The stress at the end of the step now becomes

$$
S_{i j}^{n+1}=S_{i j}^{n}+\dot{S}_{i j}\left(S_{i j}^{n+1}\right) \Delta t
$$


We choose to write the stress rate term, $\dot{\mathrm{S}}_{\mathrm{ij}}$, as

$$
\dot{S}_{i j}=\left(2 \mu\left(\Theta^{n+1}\right) \dot{\varepsilon}_{i j}+2 \dot{\mu}\left(\Theta^{n+1}\right) \varepsilon_{i j}^{n}\right)
$$

with the expression for $\dot{\mu}\left(\Theta^{n+1}\right)$ defined as

$$
\mu\left(\Theta^{n+1}\right)=\frac{\mu\left(\Theta^{n+1}\right)-\mu\left(\Theta^{n}\right)}{\Delta t}
$$

This definition for the stress increment is neither a pure forward or backward Euler representation but a hybrid method where we choose to use the total strain at the beginning of the step along with a backward difference for $\dot{\mu}\left(\Theta^{n+1}\right)$. If we employ the fact that $\varepsilon_{i j}^{n}=\frac{s_{i j}^{n}}{2 \mu\left(\Theta^{n}\right)}$, we can write the final expression for the deviatoric stress as

$$
S_{i j}^{n+1}=S_{i j}^{n}\left(\frac{\mu\left(\Theta^{n+1}\right)}{\mu\left(\Theta^{n}\right)}\right)+2 \mu\left(\Theta^{n+1}\right) \dot{\varepsilon}_{i j} \Delta t
$$

The temperature-dependent elastic bulk response is computed in a similar fashion. The equation for the bulk response at step $n+1$ is

$$
\sigma_{\mathrm{kk}}^{\mathrm{n+1}}=3 \mathrm{~K}\left(\Theta^{\mathrm{n}+1}\right) \varepsilon_{\mathrm{kk}}^{\mathrm{n}+1}
$$

We can write the above equation in an incremental form that is more suitable for numerical implementation.

$$
\sigma_{\mathrm{kk}}^{\mathrm{n}+1}=\sigma_{\mathrm{kk}}^{\mathrm{n}}\left(\frac{\mathrm{K}\left(\Theta^{\mathrm{n}+1}\right)}{K\left(\Theta^{\mathrm{n}}\right)}\right)+3 K\left(\Theta^{\mathrm{n}+1}\right) \dot{\varepsilon}_{\mathrm{kk}} \Delta \mathrm{t}
$$

The stress from the deviatoric and bulk responses is combined to give the final stress state.

The thermoelastic material uses the following state variables:

YMO - Young's modulus at the beginning of the step

YM1 - Young's modulus at the end of the step

XNU0 - Poisson's ratio at the beginning of the step

XNU1 - Poisson's ratio at the end of the step.

The PROP array for this material contains the following entries:

PROP(1) - Young's modulus, E

PROP(2) - Poisson's ratio, v 
PROP(3) - Modulus function identification

PROP(4) - Poisson's ratio function identification.

\subsection{Thermoelastic-Plastic Power Law Hardening Material Model}

This material model represents the behavior of an elastic-plastic power law hardening material with temperaturedependent material constants. Both Young's modulus and Poisson's ratio are allowed to vary with temperature along with the material yield stress. The values of Young's modulus, Poisson's ratio, and yield stress at the beginning and end of the time step are stored as state variables. The relationship between the material property value and temperature is specified using a FUNCTION definition.

We will separate the material behavior into deviatoric and bulk responses. If we consider only deviatoric behavior, the model has the following form during a plastic loading process

$$
\bar{\sigma}=\sigma_{y s}(\Theta)+A\left\langle\bar{\varepsilon}_{p}-\varepsilon_{L}\right\rangle^{m}
$$

where $\mathrm{A}$ and $\mathrm{m}$ are material constants, $\sigma_{\mathrm{ys}}$ is the temperature-dependent initial yield stress, $\bar{\varepsilon}_{\mathrm{p}}$ is the equivalent plastic strain, $\bar{\sigma}$ is the effective stress, and $\varepsilon_{L}$ is the Lüders strain or yield plateau strain. The use of brackets in the above expression denotes the use of a Heaviside function. The function is zero until the arithmetic expression within the brackets becomes positive. The material constants for this model can be determined from measured stress versus strain data through simple curve-fitting procedures. By suitably choosing the material constants $\mathrm{A}$ and $\mathrm{m}$, the form of the model can represent either elastic/perfectly plastic or linear hardening material behavior in addition to the power law hardening response. The temperature dependence is captured by allowing the initial yield stress and elastic constants to be a function of temperature.

The expression for the stress at the end of the step is

$$
\mathrm{S}_{\mathrm{ij}}^{\mathrm{n}+1}=\mathrm{S}_{\mathrm{ij}}^{\mathrm{n}}+\dot{S}_{\mathrm{ij}}\left(\dot{S}_{\mathrm{ij}}^{\mathrm{n}+1}\right) \Delta \mathrm{t}
$$

where we have used a backward Euler integration for the stress. The stress rate at the end of the step, $\dot{S}_{i j}^{n+1}$, is defined as follows

$$
\dot{S}_{i j}=2 \mu\left(\Theta^{n+1}\right)\left(\varepsilon_{i j}^{n}-\varepsilon_{p_{i j}}^{n}\right)+2 \mu\left(\Theta^{n+1}\right)\left(\dot{\varepsilon}_{i j}-\dot{\varepsilon}_{p_{i j}}\right)
$$

where $\varepsilon_{\mathrm{p}_{\mathrm{ij}}}$ are the components of the plastic strain. We choose to use this expression for the stress rate because in the absence of plastic strain we recover the same expression for the stress as derived for the thermoelastic model. We define the difference expression for $\dot{\mu}\left(\Theta^{n+1}\right)$ as

$$
\dot{\mu}\left(\Theta^{n+1}\right)=\frac{\mu\left(\Theta^{n+1}\right)-\mu\left(\Theta^{n}\right)}{\Delta t} .
$$


For von Mises flow, we can write the above as

$$
\dot{S}_{i j}=\left(\frac{\mu\left(\Theta^{n+1}\right)-\mu\left(\Theta^{n}\right)}{\Delta t}\right)\left(\frac{S_{i j}^{n}}{\mu\left(\Theta^{n}\right)}\right)+2 \mu\left(\Theta^{n+1}\right) \dot{\varepsilon}_{i j}-2 \mu\left(\Theta^{n+1}\right)\left(\frac{3 \gamma}{2} \frac{S_{i j}^{n+1}}{\sigma^{n+1}}\right)
$$

where $\gamma$ is a scalar quantity.

Substituting the above expression for $\dot{S}_{i j}^{n+1}$ into the expression for $S_{i j}^{n+1}$ results in

$$
S_{i j}^{n+1}=S_{i j}^{n}\left(\frac{\mu\left(\Theta^{n+1}\right)}{\mu\left(\Theta^{n}\right)}\right)+2 \mu\left(\Theta^{n+1}\right) \dot{\varepsilon}_{i j} \Delta t-3 \mu\left(\Theta^{n+1}\right) \frac{S_{i j}^{n+1}}{\bar{\sigma}^{n+1}} \Delta \gamma
$$

where $\Delta \gamma=\gamma \Delta t$. The first two expressions on the right-hand-side of the equation define the trial stress $S_{i j}^{t r}$ so that the final expression becomes

$$
S_{i j}^{n+1}=S_{i j}^{t r}-\left(3 \mu\left(\Theta^{n+1}\right) \frac{S_{i j}^{n+1}}{\bar{\sigma}^{n+1}} \Delta \gamma\right)
$$

We make use of the following identity

$$
\frac{S_{\mathrm{ij}}^{\mathrm{n}+1}}{\bar{\sigma}^{\mathrm{n}+1}}=\frac{S_{\mathrm{ij}}^{\mathrm{tr}}}{\bar{\sigma}^{\mathrm{tr}}}
$$

to get the final expression for the stress at the end of the step

$$
\mathrm{S}_{\mathrm{ij}}^{\mathrm{n}+1}=\mathrm{S}_{\mathrm{ij}}^{\mathrm{tr}}\left(1-\frac{3 \mu\left(\Theta^{\mathrm{n}+1}\right) \Delta \gamma}{\bar{\sigma}^{\mathrm{tr}} \cdot}\right)
$$

The plastic strain increment, $\Delta \gamma$, is the only unknown in the equation. To solve for the plastic strain increment, we must go back to our expression for the yield function. Combining the yield function with the expression for $\mathrm{S}_{\mathrm{ij}}^{\mathrm{n}+1}$ and making use of the identity, we get

$$
\bar{\sigma}^{\operatorname{tr}}-3 \mu\left(\Theta^{n+1}\right) \Delta \gamma=\sigma_{y s}\left(\Theta^{n+1}\right)+A\left\langle\bar{\varepsilon}_{p}^{n}+\Delta \gamma-\varepsilon_{L}\right\rangle^{m}
$$

which is solved for $\Delta \gamma$ using Newton Raphson iteration. The computed value of $\Delta \gamma$ is substituted back into the expression for $S_{i j}^{n+1}$, Equation (4.11.9).

The bulk response is assumed to be linear elastic. The pressure at the end of the step is calculated using the volumetric strain, $\varepsilon_{\mathrm{kk}}$, through the relation 


$$
\sigma_{\mathrm{kk}}^{\mathrm{n}+1}=3 \mathrm{~K}\left(\Theta^{\mathrm{n}+1}\right) \varepsilon_{\mathrm{kk}}^{\mathrm{n}+1}
$$

where $K\left(\Theta^{n+1}\right)$ is the temperature-dependent bulk modulus at the end of the step. Following the procedure outlined for the thermoelastic material model, we can arrive at the final expression for the bulk response.

$$
\sigma_{k k}^{n+1}=\sigma_{k k}^{n}\left(\frac{K\left(\Theta^{n+1}\right)}{K\left(\Theta^{n}\right)}\right)+3 K\left(\Theta^{n+1}\right) \dot{\varepsilon}_{k k} \Delta t
$$

The stress from the deviatoric and bulk responses is combined to give the final stress state.

The thermoelastic-plastic material uses the following state variables:

$\begin{array}{ll}\text { EQPS } & \text { - Equivalent plastic strain } \\ \text { YM0 } & \text { - Young's modulus at the beginning of the step } \\ \text { YM1 } & \text { - Young's modulus at the end of the step } \\ \text { XNU0 } & \text { - Poisson's ratio at the beginning of the step } \\ \text { XNU1 } & \text { - Poisson's ratio at the end of the step } \\ \text { YSO } & \text { - Yield stress at the beginning of the step } \\ \text { YS1 } & \text { - Yield stress at the end of the step } \\ \text { RADIUS } & \text { - Radius of the yield surface. }\end{array}$

The PROP array for this material contains the following entries:

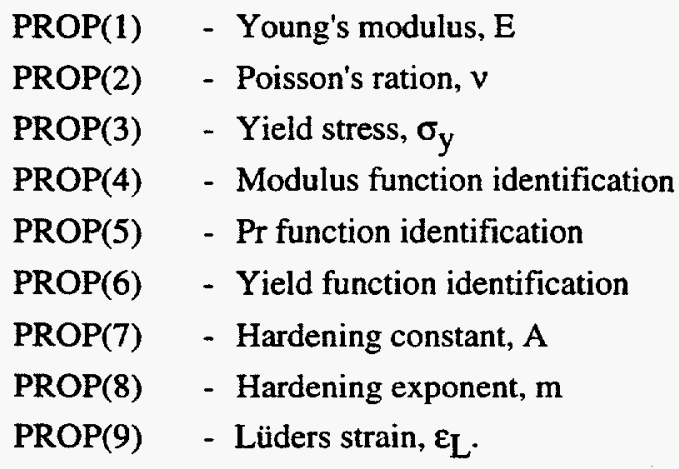

\subsection{Multi-mechanism Deformation (M-D) Creep Model}

A multi-mechanism deformation (M-D) model proposed by Munson and Dawson (1979, 1982, 1984) and extended by Munson et al. (1988) has been included in SANTOS to model the creep behavior of rock salt. This model, which is based on the deformation map for salt, describes the relationship between the steady-state creep rate; stress, and temperature in terms of three deformation mechanisms in salt. Two of these mechanisms are dislocation glide and dislocation climb. The effects of a third mechanism are also included in the M-D model. Although this mechanism has not been characterized in terms of microstructural processes, its effects are observed in creep 
experiments. Transient workhardening and recovery responses are incorporated through a state variable function that modifies the steady-state creep rates.

In the M-D model, the equivalent creep strain rate at steady-state, $\dot{\varepsilon}_{S}$, is assumed to be equal to the sum of three terms, each arising from one of the three mechanisms described above, i.e.,

$$
\dot{\varepsilon}_{\mathrm{s}}=\dot{\varepsilon}_{\mathrm{s} 1}+\dot{\varepsilon}_{\mathrm{s} 2}+\dot{\varepsilon}_{\mathrm{s} 3}
$$

The three equivalent strain rates appearing on the right-hand-side of the above equation are given by the following functions:

$$
\begin{gathered}
\dot{\varepsilon}_{\mathrm{s} 1}=\mathrm{A}_{1} \exp \left(-\frac{\mathrm{Q}_{1}}{\mathrm{R} \Theta}\right)\left(\frac{\sigma_{\mathrm{e}}}{\mu}\right)^{\mathrm{n}_{1}} \\
\dot{\varepsilon}_{\mathrm{s} 2}=\mathrm{A}_{2} \exp \left(-\frac{\mathrm{Q}_{2}}{\mathrm{R} \Theta}\right)\left(\frac{\sigma_{\mathrm{e}}}{\mu}\right)^{\mathrm{n}_{2}} \\
\dot{\varepsilon}_{\mathrm{s} 3}=\mathrm{H}\left(\sigma_{\mathrm{e}}-\sigma_{\mathrm{o}}\right)\left[\mathrm{B}_{1} \exp \left(-\frac{\mathrm{Q}_{1}}{\mathrm{R} \Theta}\right)+\mathrm{B}_{2} \exp \left(-\frac{\mathrm{Q}_{2}}{\mathrm{R} \Theta}\right)\right] \sinh \left(\frac{\mathrm{q}\left(\sigma_{\mathrm{e}}-\sigma_{\mathrm{o}}\right)}{\mu}\right)
\end{gathered}
$$

where the $A_{i}$ 's, $B_{i}$ 's and $n_{i}$ 's are constants, the $Q_{i}$ 's are activation energies, $\Theta$ is the absolute temperature, $R$ is the universal gas constant, $\mu$ is the elastic shear modulus, $\sigma_{e}$ is the equivalent stress, $q$ is a constant, $\sigma_{o}$ is the stress limit of the dislocation slip mechanism, and $\mathrm{H}$ is the Heaviside step function. In the above equation, $\dot{\varepsilon}_{\mathrm{s} 1}$ represents the effects of the dislocation climb mechanism, $\dot{\varepsilon}_{\mathrm{s} 2}$ represents the effects of the unidentified mechanism, and $\dot{\varepsilon}_{\mathrm{s} 3}$ represents the effects of the glide mechanism. The relationship between $\sigma_{\mathrm{e}}$ and the components of the stress tensor under general loading conditions depends on the choice of the stress generalization and will be discussed later.

Transient creep is incorporated in the M-D model through the use of a scaling function F, which modifies the steady-state creep rate. The total equivalent creep rate $\dot{\varepsilon}$ is given by:

$$
\dot{\varepsilon}=\mathrm{F}\left(\sigma_{\mathrm{e}}, \Theta, \varsigma\right) \dot{\varepsilon}_{\mathrm{s}}
$$

The arguments of the transient scaling function are equivalent stress, temperature, and an internal state variable, $\zeta$. The evolution of $\zeta$ is described by a separate rate equation. Three branches of the function $\mathrm{F}$ can be identified: (1) a workhardening branch where $F$ assumes a value greater than unity, (2) an equilibrium branch where $F$ is equal to unity, and (3) a recovery branch where $F$ is less than unity. The expression for $F$ is

$$
\begin{gathered}
F=\exp \left(\left[1-\frac{\zeta}{\varepsilon_{t}^{*}}\right]^{2} \Delta\right) \text { for } \zeta<\varepsilon_{t}^{*} \\
F=1 \text { for } \zeta=\varepsilon_{t}^{*}
\end{gathered}
$$




$$
F=\exp \left(-\left[1-\frac{\zeta}{\varepsilon_{t}^{*}}\right]^{2} \delta\right) \text { for } \zeta>\varepsilon_{t}^{*}
$$

In the above equations, $\Delta$ and $\delta$ are referred to as the workhardening and recovery functions, respectively, while $\varepsilon_{t}^{*}$ is referred to as the transient strain limit. The workhardening and recovery functions are assumed to be of the form

$$
\begin{aligned}
& \Delta=\alpha_{w}+\beta_{w} \log \left(\frac{\sigma_{\mathrm{e}}}{\mu}\right) \\
& \delta=\alpha_{\mathrm{r}}+\beta_{\mathrm{r}} \log \left(\frac{\sigma_{\mathrm{e}}}{\mu}\right) .
\end{aligned}
$$

The transient strain limit is a function of temperature and stress given by

$$
\varepsilon_{\mathrm{t}}^{*}=\mathrm{K}_{0} \exp (\mathrm{c} \Theta)\left(\frac{\sigma_{\mathrm{e}}}{\mu}\right)^{\mathrm{m}}
$$

where $\mathrm{K}_{0}$ and $\mathrm{c}$ are constants. Finally, the evolution equation for the internal variable $\zeta$ is

$$
\dot{\zeta}=(F-1) \dot{\varepsilon}_{S}=\dot{\varepsilon}-\dot{\varepsilon}_{S}
$$

To complete the generalization of the M-D constitutive model, an equivalent stress and flow rule for the creep strain rate must be defined. These two definitions provide the necessary linkage among the three-dimensional stress state, the creep strain rate, and the invariant creep relationships described earlier. According to Munson et al. (1988), the Tresca stress generalization provides the most appropriate definition of the equivalent stress for rock salt. With the Tresca stress generalization, the equivalent stress becomes

$$
\sigma_{\mathrm{e}}^{\mathrm{t}}=2 \sqrt{\mathrm{J}_{2}} \cos \psi=\sigma_{1}-\sigma_{3}
$$

where $\psi$ is the Lode angle defined by

$$
\sin 3 \psi=\frac{-3 J_{3} \sqrt{3}}{2 J_{2}^{3 / 2}}
$$

In the two preceding equations, $\mathrm{J}_{2}$ and $\mathrm{J}_{3}$ are the second and third invariants of the deviatoric part of the stress tensor, and $\sigma_{1}$ and $\sigma_{3}$ are the maximum and minimum principal stresses, respectively. The flow is assumed to be associative so that the direction of $\dot{\bar{\varepsilon}}$ is normal to the Tresca flow surface. Unfortunately, the normal is undefined as $\psi= \pm \frac{\pi}{6}$ where sharp corners exist in the Tresca flow surface. At these locations, the flow is assumed to be normal to the von Mises flow surface. The von Mises flow surface is characterized by a constant value of $\sigma_{\mathrm{e}}^{\mathrm{vm}}$ where 


$$
\sigma_{\mathrm{e}}^{\mathrm{vm}}=\sqrt{3 \mathrm{~J}_{2}}
$$

Note that $\sigma_{\mathrm{e}}^{\mathrm{vm}}=\sigma_{\mathrm{e}}^{\mathrm{t}}$ for $\Psi=\frac{\pi}{6}$.

The expression for the effective or equivalent creep strain rate, $\dot{\bar{\varepsilon}}_{\mathrm{ij}}^{\mathrm{c}}$, for Tresca flow is

$$
\dot{\bar{\varepsilon}}_{\mathrm{ij}}^{c}=\dot{\varepsilon}\left[\frac{\cos 2 \theta}{\cos 3 \theta \sqrt{\mathrm{J}_{2}}} \mathrm{~S}_{\mathrm{ij}}+\frac{\sin \theta \sqrt{3}}{\cos 3 \theta \mathrm{J}_{2}}\left(\mathrm{~S}_{\mathrm{ip}} \mathrm{S}_{\mathrm{pj}}-\frac{2 \mathrm{~J}_{2}}{3} \delta_{\mathrm{ij}}\right)\right]
$$

where $\dot{\bar{\varepsilon}}$ can be replaced by $F \dot{\varepsilon}_{\mathbf{S}}$. The resulting expression for the stress rate, $\dot{S}_{\mathrm{ij}}^{\mathrm{n}}$, now becomes

$$
\dot{S}_{\mathrm{ij}}^{\mathrm{n}}=2 \mu\left(\dot{\varepsilon}_{\mathrm{ij}}-\mathrm{F} \dot{\varepsilon}_{\mathrm{s}}\left[\frac{\cos 2 \theta}{\cos 3 \theta \sqrt{\mathrm{J}_{2}}} \mathrm{~S}_{\mathrm{ij}}^{\mathrm{n}}+\frac{\sin \theta \sqrt{3}}{\cos 3 \theta \mathrm{J}_{2}}\left(\mathrm{~S}_{\mathrm{ip}}^{\mathrm{n}} \mathrm{S}_{\mathrm{pj}}^{\mathrm{n}}-\frac{2 \mathrm{~J}_{2}}{3} \delta_{\mathrm{ij}}\right)\right]\right)
$$

which is highly nonlinear.

Integration of this equation and the evolutionary equation governing the rate of change of $\zeta$ requires the use of a numerical procedure. Studies of various numerical integration methods have revealed that simple forward Euler integration is as effective as any method. Following the methodology outlined previously for the Power Law Creep Model, the stress at step $\mathrm{n}+1$ is simply

$$
S_{i j}^{n+1}=S_{i j}^{n}+\dot{S}_{i j}\left(S_{i j}^{n}\right) \Delta t
$$

and in a similar fashion

$$
\zeta^{n+1}=\varsigma^{n}+\dot{\zeta}^{n}\left(S_{i j}^{n}\right) \Delta t
$$

The forward Euler operator is conditionally stable. The critical time step for stability can be determined using the method of Cormeau (1975) based on the form of the flow potential and the elastic constants. This has been done for the M-D model. Two estimates for the critical time step are computed, and the minimum of the two is used for the calculation. These time step estimates are given by

$$
\Delta \mathrm{t}_{\mathrm{st1}} \leq \frac{1}{\mu\left(\frac{\partial \dot{\bar{\varepsilon}}^{\mathrm{c}}}{\partial \tau_{\max }}\right)}
$$

and

$$
\Delta \mathrm{t}_{\mathrm{st} 2} \leq \frac{-\sqrt{3}}{\left(\frac{\partial \dot{\bar{\varepsilon}}^{\mathrm{c}}}{\partial \zeta}\right)}
$$

where $\tau_{\max }=\cos \theta \sqrt{\mathrm{J}_{2}}=\frac{\bar{\sigma}}{2}$. The equivalent Tresca stress, $\bar{\sigma}$, is the value at the end of step $n$. 
Accuracy of the method is assured if the time steps are sufficiently small, but using the stable time step cannot be guaranteed to always produce an accurate solution. However, comparison of results using this integration method with known solutions for complex two- and three-dimensional creep problems has shown the method to be very accurate when the stable time step is used.

For a typical application, the time increment, $\Delta \mathrm{t}_{\mathrm{st} 1}$, is smallest when $\bar{\sigma}$ is largest, and as the effective stress decreases, such as when the problem approaches steady-state creep, the critical time step increases. In most instances, the user-specified time step, $\Delta \mathrm{t}$, will be larger than the time step needed for stability, $\min \left(\Delta \mathrm{t}_{\mathrm{st}}, \Delta \mathrm{t}_{\mathrm{st} 2}\right)$. Therefore, the solution time step is broken into subincrements for integrating the constitutive model with the size of each subincrement changing with the stress. The subincrementation procedure is discussed at the beginning of this chapter.

The transient creep part of the M-D model causes the stress to change rapidly, which causes the time step to be very small. The adaptive time step feature was developed to accommodate the small time step initially and allow it to grow as the solution proceeds toward steady state. Experience has shown that an initial time step size of $1.0 \times 10^{-}$ 5 seconds works well with a tolerance of 0.01 . The maximum time step size depends on the time scale of the problem and the degree of nonlinearity.

The M-D creep model uses the following state variables:

$$
\begin{array}{ll}
\text { EQCS } & \text { - equivalent creep strain } \\
\text { ZETA } & \text { - current values of the evolutionary parameter } \\
\text { SDOT } & \text { - stress rate for the current time step } \\
\text { TRESCA } & \text { - equivalent stress computed using TRESCA criterion } \\
\text { ETSTAR } & \text { - transient strain limit. }
\end{array}
$$

The PROP array for this material contains the following entries:

$$
\begin{array}{ll}
\text { PROP(1) } & -2 \mu \\
\text { PROP(2) } & -\mathrm{K} \\
\text { PROP(3) } & -\mathrm{A} 1 \\
\text { PROP(4) } & -\frac{\mathrm{Q} 1}{\mathrm{R}} \\
\mathrm{PROP}(5) & -\mathrm{n} 1 \\
\mathrm{PROP}(6) & -\mathrm{B} 1 \\
\mathrm{PROP}(7) & -\mathrm{A} 2 \\
\mathrm{PROP}(8) & -\frac{\mathrm{Q} 2}{\mathrm{R}} \\
\mathrm{PROP}(9) & -\mathrm{n} 2 \\
\mathrm{PROP}(10) & -\mathrm{B} 2 \\
\mathrm{PROP}(11) & -\sigma_{0} \\
\mathrm{PROP}(12) & -\mathrm{q} \\
\mathrm{PROP}(13) & -\mathrm{m} \\
\mathrm{PROP}(14) & -\mathrm{K}_{0} \\
\text { PROP(15) } & -\mathrm{c} \Theta
\end{array}
$$




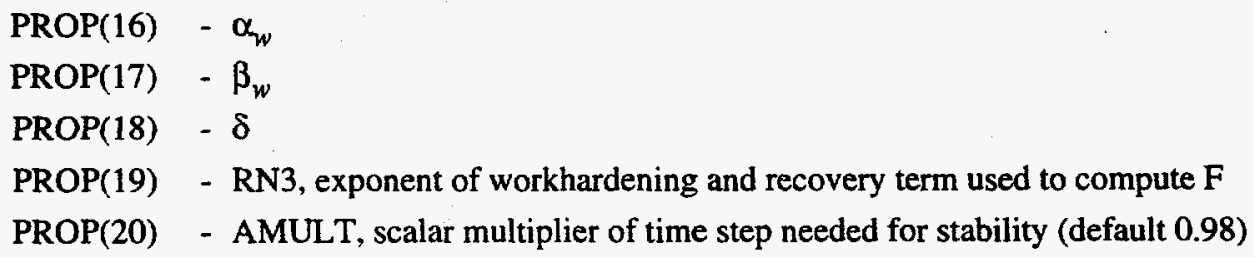

\subsection{Volumetric Creep Model}

The consolidation behavior of geomaterials and salt, in particular, is of interest to analysts because of the use of these materials as backfill and as a sealing material in waste disposal applications. The volumetric creep model implemented in SANTOS is based on the work of Sjaardema and Krieg (1987), who developed their model based on the hydrostatic consolidation tests of salt with added water by Holcomb and Shields (1987): Time-dependent behavior is included in both the volumetric and the deviatoric response. The form of the model is such that the mechanical response of the consolidated material becomes identical to that of the intact material as the density approaches that of the intact material. The elastic moduli were found from the tests to depend on the density, $\rho$, of the material through relationships of the form

$$
\begin{gathered}
K=K_{0} \exp \left(K_{1} \rho\right) \\
\mu=\mu_{0} \exp \left(\mu_{1} \rho\right)
\end{gathered}
$$

where $K_{0}, K_{1}, \mu_{0}$, and $\mu_{1}$ are material constants.

For the discussion of the volumetric creep model, it is appropriate to decompose the total strain rate into volumetric and deviatoric parts. Because intact salt creeps deviatorically when subjected to a deviatoric stress state, crushed salt should logically be expected to creep deviatorically. This expectation becomes more reasonable as the density increases because, in the limit, the crushed salt becomes intact salt. The deviatoric crushed salt creep model presented here is based on the power law secondary creep model, which has been used to describe the creep behavior of intact salt. This model is described in Section 4.9, Power Law Creep Material Model.

The development proceeds by envisioning that the porous crushed salt uniaxial sample is composed of cylinders of salt, each of which has the intact salt secondary creep behavior separated by areas of open space. The local stress acting on the salt cylinders is then stated in terms of the average stress on the porous sample. The cross-sectional area of the porous sample is expressed in terms of the net cross-sectional area of the salt cylinders. The final resulting continuum model for the rate of the deviatoric stress of crushed salt is then

$$
\dot{S}_{\mathrm{ij}}^{n}=2 \mu(\rho)\left(\dot{\varepsilon}_{\mathrm{ij}}-\frac{3}{2} \mathrm{~A}\left(\frac{\rho_{\infty}}{\rho}\right)^{\mathrm{m}} \exp \left(\frac{-Q}{R \Theta}\right) \bar{\sigma}^{\mathrm{m}-1} S_{\mathrm{ij}}^{\mathrm{n}}\right)
$$

where the constants $A, Q, m$, and $\rho_{\infty}$ refer to values for the intact material. The integration of the deviatoric part of the stress is performed using the forward Euler operator. The integration method is the same as used for the power law creep model, and the details of the integration may be found in Section 4.9, Power Law Creep Material Model. 
The volumetric part of the model can be written as the sum of elastic and inelastic parts as shown below

$$
\mathrm{d}_{\mathrm{kk}}=\frac{\dot{\mathrm{p}}}{\mathrm{K}(\rho)}+\mathrm{d}_{\mathrm{kk}}^{\mathrm{c}}
$$

where $d_{k k}$ is the volumetric strain rate, $\dot{p}=\frac{\dot{\sigma}_{k k}}{3}$ is the rate of change of the pressure, $d_{k k}^{c}$ is the volumetric creep strain rate, and $K .(\rho)$ is the density-dependent bulk modulus. . Laboratory consolidation tests on crushed salt have shown the volumetric creep strain rate to be fit well by an expression of the form

$$
d_{k k}^{c}=\frac{1}{\rho} B_{0}\left[e^{B_{1} p}-1\right] e^{A \rho}
$$

where $B_{0}, B_{1}$, and $A$ are material constants obtained from the experiments. The density $\rho$ is computed from the relationship

$$
\rho=\rho_{0} \exp \left(\int_{t_{0}}^{t} d_{k k} d t\right)
$$

where $\rho_{0}$ is the density at time $t_{0}$. Equation (4.13.4) is solved for $\dot{\mathrm{p}}$ and combined with the definition of the volumetric creep strain rate, Equation (4.13.5), to produce

$$
\dot{p}=K(\rho)\left[d_{k k}-\frac{1}{\rho} B_{0}\left(e^{B_{1} p}-1\right) e^{A \rho}\right]
$$

which is the expression to be integrated.

The expression for the pressure is integrated using the backward Euler operator. This operator is unconditionally stable for any time step size. The expression for the pressure at the end of the step is

$$
\mathrm{p}^{\mathrm{n}+1}=\mathrm{p}^{\mathrm{n}}+\dot{\mathrm{p}}^{\mathrm{n}+1} \Delta \mathrm{t}
$$

which can be rewritten using the above equation as

$$
p^{n+1}=p^{n}+\left(K\left(\rho^{n+1}\right)\left[d_{k k}-\frac{1}{\rho^{n+1}} B_{0}\left(e^{B_{1} p^{n+1}}-1\right) e^{A \rho^{n+1}}\right]\right) \Delta t
$$

Let us define a trial pressure as $p^{t r}=p^{n}+K\left(\rho^{n+1}\right) d_{k k} \Delta t$, which lets us rewrite the above expression as

$$
p^{n+1}=p^{t r}-K\left(\rho^{n+1}\right)\left(\frac{1}{\rho^{n+1}} B_{0}\left(e^{B_{1} p^{n+1}}-1\right) e^{A \rho^{n+1}}\right) \Delta t
$$


We need to solve the above expression for the pressure, $\mathrm{p}^{\mathrm{n}+1}$, for which we have chosen to use a Newton Raphson scheme with a fixed number of iterations. Once we have the pressure at the end of the step, it is combined with the deviatoric stresses to produce a trial stress state for the material. The trial stresses are accepted as the final stresses if: (1) the mean stress is tensile; (2) the out-of-plane trial stress is compressive; or (3) the mean stress is compressive, and the out-of-plane trial stress is tensile but is less than $10 \%$ of the absolute value of the mean stress. If these conditions are not met, then the deviatoric stresses are scaled back so that the out-of-plane stress is equal to $10 \%$ of the absolute value of the mean stress.

The volumetric creep material model uses the following state variables:

$$
\begin{array}{ll}
\text { EQCS } & \text { - equivalent creep strain } \\
\text { DENSITY } & \text { - current density of the consolidating material. }
\end{array}
$$

The PROP array for this material contains the following entries:

$$
\begin{array}{ll}
\text { PROP(1) } & -2 \mu \\
\text { PROP(2) } & -\mathrm{K} \\
\text { PROP(3) } & -\mathrm{A}, \text { creep constant } \\
\text { PROP(4) } & -\mathrm{m} \text { stress exponent } \\
\text { PROP(5) } & -\frac{\mathrm{Q}}{\mathrm{R} \Theta} \text { if isothermal or } \frac{\mathrm{Q}}{\mathrm{R}} \text { if not } \\
\mathrm{PROP}(6) & -\mu_{1}, \text { shear exponent } \\
\mathrm{PROP}(7) & -\mathrm{K}_{1}, \text { bulk exponent } \\
\mathrm{PROP}(8) & -\mathrm{B}_{0} \\
\mathrm{PROP}(9) & -\mathrm{B}_{1} \\
\mathrm{PROP}(10) & -\mathrm{A}_{1} \\
\mathrm{PROP}(11) & -P_{\text {intact }}, \text { intact density } \\
\text { PROP(12) } & -\rho_{0}, \text { initial density. }
\end{array}
$$

\subsection{Viscoelastic Material Model}

The mechanical response of many plastics, rubbers, epoxies, glasses, and other polymeric compounds can be described quite well by a linear viscoelastic constitutive law. In the absence of any changes in temperature, the stress at time $t$ in a linear material with memory depends only on the past strain history. This can be expressed in general terms as

$$
S_{i j}=2 \int_{-\infty}^{t} G(t-\tau) \dot{\varepsilon}_{i j}(\tau) d \tau
$$

for the shear response and

$$
\sigma_{k k}=3 \int_{-\infty}^{t} K(t-\tau) \dot{\varepsilon}_{k k}(\tau) d \tau
$$


for the bulk response, where $G(t)$ and $K(t)$ are the shear and bulk relaxation moduli, respectively.

Unlike elastic constitutive equations in which the material moduli are constants, viscoelastic relations employ moduli that relax over some period of time. A specific form for the relaxation moduli is obtained by considering the mechanical analogy of the standard linear viscoelastic solid shown in Figure 4.14.1. The springs represent the elastic response and the dashpots represent the viscous response. By stringing together $\mathrm{N}$ such elements the relaxation moduli can be written as (Ferry, 1970)

$$
G(t)=G^{\infty}+\sum_{\alpha=1}^{N_{s}}\left(G_{\alpha}-G^{\infty}\right) e^{-\beta_{\alpha}^{s}(t)}
$$

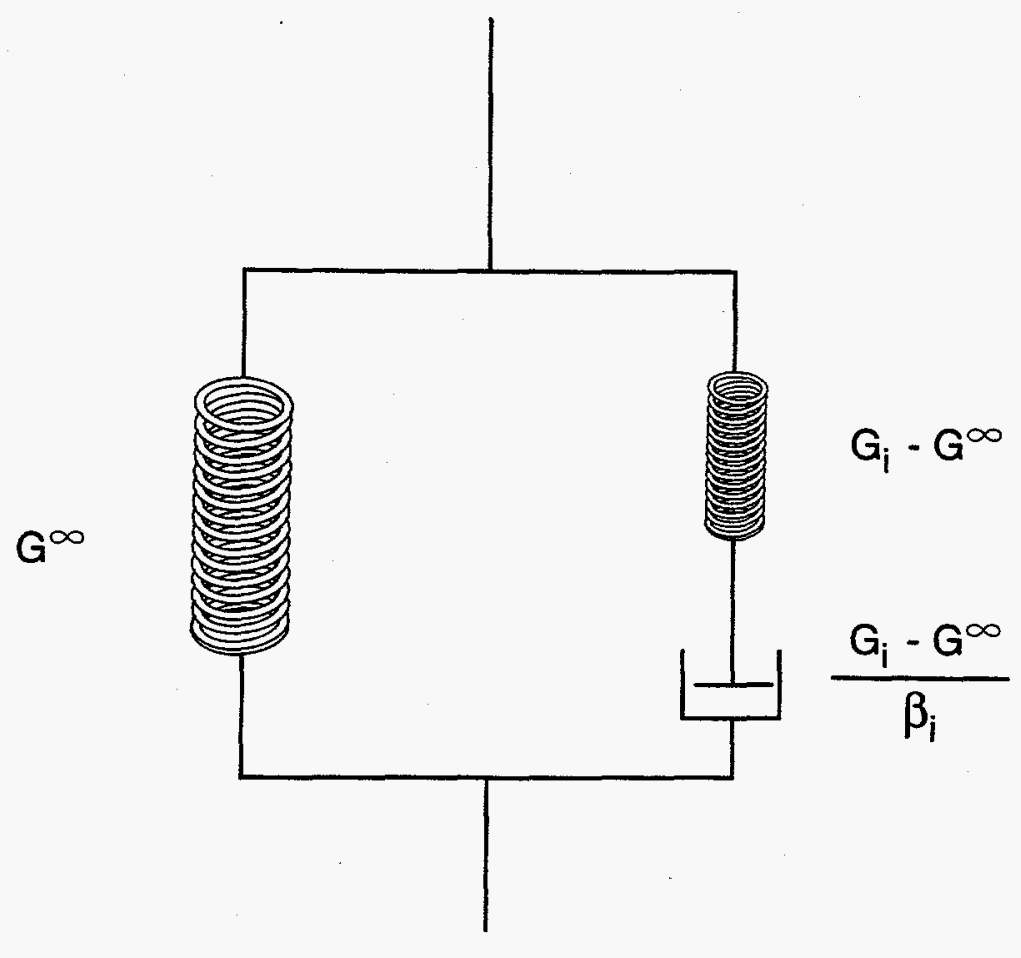

Figure 4.14.1 Mechanical analogy of the standard linear solid. 
and

$$
K(t)=K^{\infty}+\sum_{\alpha=1}^{N_{b}}\left(K_{\alpha}-K^{\infty}\right) e^{-\beta_{\alpha}^{b}(t)}
$$

where the $\beta$ 's are relaxation constants $\left(1 / \beta=\right.$ relaxation time) and $\mathrm{G}^{\infty}$ and $\mathrm{K}^{\infty}$ are the long-time moduli. Since the bulk and shear behaviors are assumed to be independent, $N_{b}$ may be different from $N_{s}$ and the same goes for $\beta^{b} s$ and the $\beta^{S}$ 's. Ideally, an arbitrary number of elements could be used to gain the most accurate representation of the behavior of the material. However, because of computer storage considerations, modeling of the bulk response is limited to one term while the shear response is limited to a three-term representation. It should be noted that Equations (4.14.3) and (4.14.4) differ slightly from the usual representation in that the long-time modulus is subtracted from each relaxation modulus. This was done to simplify the data format, but caution should be used in determining material property data for the material model to ensure that it conforms to the form of Equations (4.14.3) and (4.14.4).

The constitutive law discussed above is based on the assumption that the entire body remains at a uniform temperature. The relaxation moduli and the material parameters necessary to evaluate them can be regarded as having been determined at a base-line temperature, $\Theta_{0}$. To evaluate the effects of temperature changes, first let us consider the modifications to the constitutive law if a uniform change in temperature is allowed. To do this, let $G(t$, $\Theta)$ be the shear relaxation modulus at the constant temperature, $\Theta$. The remainder of the theory will be developed using the shear modulus as an example. The modifications to the bulk modulus are handled in the same manner and will not be repeated.

Using a change of variable, the shear modulus at the base-line temperature can be written as a function of $\log \mathrm{t}$. We can now apply the hypothesis of temperature-time equivalence (Ferry, 1970; Leaderman, 1943; Ferry, 1950), which states that all response functions (e.g., relaxation moduli) are affected by the uniform temperature change only within a corresponding uniform shift of the logarithmic time scale. Materials exhibiting this kind of behavior have come to be termed "thermorheologically simple" materials (Schwarzl and Staverman, 1952) This leads to the following form for the relaxation moduli:

$$
G(t, \Theta)=L[\log t+\Psi(\Theta)]
$$

where $\Psi(\Theta)$ is the "shift function" and is usually written as

$$
\Psi(\Theta)=\log \varphi(\Theta)
$$

where the "shift factor," $\varphi(\Theta)$, conforms to

$$
\varphi\left(\Theta_{0}\right)=1, \varphi(\Theta)>0, \frac{\mathrm{d}}{\mathrm{d} \Theta} \varphi>0
$$

If we now define a "reduced time" by

$$
\xi=t \varphi(\Theta)
$$


then

$$
G(t, \Theta)=G(\xi)
$$

This means that the entire family of response functions can be determined by one member $G(t)=G\left(t, \Theta_{0}\right)$, provided the shift factor is known. The shift factor is assumed to be a material property that can be determined experimentally. It should be noted that the temperature dependence of the responses in shear and in bulk could be governed by two different shift functions. However, this is ruled out by the assumption of a thermorheologically simple material because the relaxation modulus in tension displays the shift property only if the bulk and shear shift functions are identical (Muki and Sternberg, 1961).

An additional modification required to account for varying temperature is that the concept of reduced time has to be redefined. Morland and Lee (1960) have shown that if the reduced time is defined as

$$
\xi(x, t)=\int_{0}^{t} \varphi[\Theta(x, \lambda)] d \lambda
$$

then a generalized relaxation integral law can be derived from Equations (4.14.3) and (4.14.4). Assuming no deformation has taken place before time, $t=0$, the constitutive model can be written in the following form:

$$
\begin{gathered}
\xi(\mathrm{x}, \mathrm{t})=\int_{0}^{\mathrm{t}} \varphi[\Theta(\mathrm{x}, \lambda)] \mathrm{d} \lambda \\
\xi^{\prime}=\xi(\mathrm{x}, \tau) \\
S_{\mathrm{ij}}(\mathrm{x}, \mathrm{t})=2 \int_{0}^{\mathrm{t}} \mathrm{G}\left(\xi-\xi^{\prime}\right) \dot{\varepsilon}_{\mathrm{ij}}(\mathrm{x}, \tau) \mathrm{d} \tau \\
\sigma_{\mathrm{kk}}(\mathrm{x}, \mathrm{t})=3 \int_{0}^{\mathrm{t}} \mathrm{K}\left(\xi-\xi^{\prime}\right) \dot{\varepsilon}_{\mathrm{kk}}(\mathrm{x}, \tau) \mathrm{d} \tau \\
\mathrm{G}\left(\xi-\xi^{\prime}\right)=\mathrm{G}^{\infty}+\sum_{\alpha=1}^{N_{s}}\left(\mathrm{G}_{\alpha}-\mathrm{G}^{\infty}\right) \mathrm{e}^{-\beta_{\alpha}^{\mathrm{s}}\left(\xi-\xi^{\prime}\right)} \\
\mathrm{K}\left(\xi-\xi^{\prime}\right)=\mathrm{K}^{\infty}+\sum_{\alpha=1}^{N_{\mathrm{b}}}\left(\mathrm{K}_{\alpha}-\mathrm{K}^{\infty}\right) \mathrm{e}^{-\beta_{\alpha}^{\mathrm{b}}\left(\xi-\xi^{\prime}\right)}
\end{gathered}
$$

For problems where changes in temperature are important, the shift factor must be specified. For convenience, a specific form of the shift factor is incorporated into the material subroutine. An empirical equation which has been 
been shown to reflect accurately the behavior of many polymers near the glass transition temperature is used. This equation, known as the WLF equation (Ferry, 1970; Williams, 1955; Williams et al., 1955) is given by

$$
\log \varphi_{10}(\Theta)=\frac{C_{1}^{0}\left(\Theta-\Theta_{0}\right)}{C_{2}^{0}+\Theta-\Theta_{0}}
$$

where $C_{1}^{0}$ and $C_{2}^{0}$ are WLF constants which have been determined for many common polymers (Ferry, 1970). If a different shift factor is available for the material of interest, it can easily be incorporated into the subroutine in place of the WLF equation.

Integration of the shear and bulk equations is done in an identical manner. The equations could, in principle, be integrated directly. However, this would require additional computer storage because the integration for each time step is over all previous history. The storage requirements for this method would be prohibitive. A better method for determining the stresses at each time step is based on the development of a recursion relation so that the stress at time $n+1$ can be determined from historical quantities at time $n$ and the current value of the strain rate. The development of this recursive method as implemented in SANTOS is described in Costin and Stone (1985). The deviatoric and the bulk stress components are combined to give the final stress state for the material.

Historical information for each stress component is stored as a state variable. The viscoelastic material has the following state variables:

$\begin{array}{lll}\text { BLKDECAY } & \text { - } & \text { single term bulk pressure } \\ \text { DECAYX1 } & \text { - } & \text { shear relaxation term } 1 \text { x-stress } \\ \text { DECAYY1 } & \text { - } & \text { shear relaxation term } 1 \text { y-stress } \\ \text { DECAYZ1 } & \text { - } & \text { shear relaxation term } 1 \text { z-stress } \\ \text { DECAYXY1 } & - & \text { shear relaxation term } 1 \text { xy-stress } \\ \text { DECAYX2 } & - & \text { shear relaxation term } 2 \text { x-stress } \\ \text { DECAYY2 } & - & \text { shear relaxation term } 2 \text { y-stress } \\ \text { DECAYZ2 } & - & \text { shear relaxation term } 2 \text { z-stress } \\ \text { DECAYXY2 } & - & \text { shear relaxation term } 2 \text { xy-stress } \\ \text { DECAYX3 } & - & \text { shear relaxation term } 3 \text { x-stress } \\ \text { DECAYY3 } & - & \text { shear relaxation term } 3 \text { y-stress } \\ \text { DECAYZ3 } & - & \text { shear relaxation term } 3 \text { z-stress } \\ \text { DECAYXY3 } & - & \text { shear relaxation term } 3 \text { xy-stress. }\end{array}$

The PROP array for this material contains the following entries:

$$
\begin{aligned}
& \text { PROP(1) - Short Time Bulk Modulus, } \mathrm{K} \\
& \text { PROP(2) - Long Time Bulk Modulus, } \mathrm{K}^{\infty} \\
& \text { PROP(3) - Bulk Relaxation Constant, } \beta^{\mathbf{k}} \\
& \text { PROP(4) - Long Time Shear Modulus, } \mathrm{G}^{\infty} \\
& \text { PROP(5) - First Short Time Shear Modulus, } \mathrm{G}_{1} \\
& \text { PROP(6) - Second Short Time Shear Modulus, } \mathrm{G}_{2}
\end{aligned}
$$


PROP(7) - Third Short Time Shear Modulus, $G_{3}$

PROP(8) - First Shear Relaxation Constant, $\beta_{1}^{\mathbf{S}}$

PROP(9) - Second Shear Relaxation Constant, $\beta_{2}^{S}$

PROP(10) - Third Shear Relaxation Constant, $\beta_{3}^{\mathbf{S}}$

PROP(11) - First WLF constant, $\mathrm{C}_{1}$

PROP(12) - Second WLF constant, $\mathrm{C}_{2}$

PROP(13) - Reference Temperature for Material Properties, $T_{0}$. 
Intentionally Left Blank 


\subsection{CONTACT SURFACES}

Many structures of interest are composed of two or more parts that are either in contact or may come into contact during service. After contact, these parts can also slide with respect to one another. In the field of computational mechanics, modeling contact and sliding behavior are done using contact surfaces, and there are several numerical approaches for modeling this behavior. One method utilizes a thin finite element with a special constitutive model to approximate gap and friction behavior (Goodman and Dubois, 1972). A second approach uses Lagrange multipliers to impose gap closure constraints and frictional stick-slip conditions (Hibbitt, Karlsson \& Sorensen, Inc., 1992). In the setting of the dynamic relaxation algorithm, SANTOS employs a sliding contact surface algorithm using a master-slave approach.

In the master-slave concept, the nodes on the designated slave surface are required by the algorithm to lie on the master surface. Any sliding or slip must occur along the master surface. In turn, nodal forces from the slave node are removed and applied to the master surface nodes. This transfer of forces maintains equilibrium at the interface. The tangential shear or friction force as well as the determination of slip or no slip is incorporated into this process involving the transfer of forces to the master surface. In SANTOS, the nodal forces are computed by the divergence of the stresses within an element. Therefore, nodal forces can be used in conjunction with a MohrCoulomb model for the contact surface friction. SANTOS currently supports two types of master-slave contact surface boundary conditions: (1) a deformable surface against a tigid plane, and (2) two distinct deformable surfaces against each other.

For a strict master-slave algorithm such as the one implemented in SANTOS, the user must specify which surface is the master surface and which surface is the slave. This choice can have a significant effect on the calculation and the efficiency of the solution. For example, the coarser mesh should be designated as the master surface when the two contacting materials are the same as shown in Figure 5.1a. If the master-slave surfaces are reversed as in Figure 5.1b, interpenetration that is not detected by the algorithm can result. The choice of masterslave roles is less clear when the materials of the contacting bodies are different. The user should typically select the stiffer of the two materials to be the master surface but be prepared to reverse the roles if convergence is slow or interpenetration is observed.

The contact surface algorithm is composed of two phases: (1) a location phase where the time, location, and amount of slave node penetration of the master surface is determined along with identification of the correct master surface segment and master surface nodes defining the segment, and (2) an application phase where the nodal force transfer from the slave node to the master surface nodes is performed along with a kinematic location of the slave node to the appropriate location on the master surface.

\subsection{Location Phase}

Contact location is accomplished by monitoring the displacements of the slave nodes throughout the calculation for possible penetration of a master surface. SANTOS uses an algorithm called neighborhood identification to pair those slave nodes and master surfaces where potential contact is likely. The neighborhood identification is usually the most time-consuming part of the location phase. Obviously, the most robust approach 


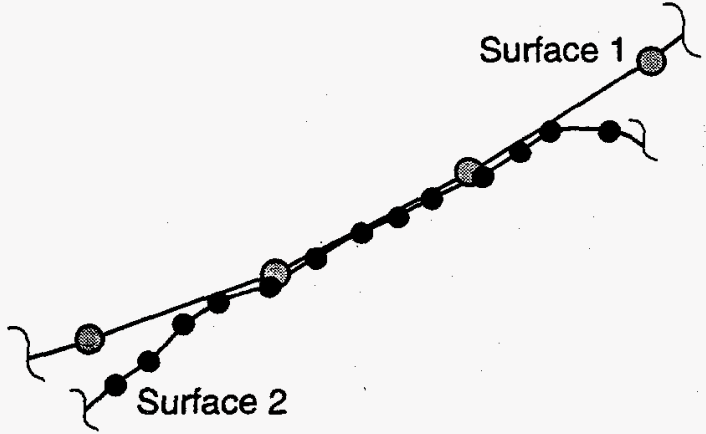

(a) Surface 1 is the master and surface 2 is the slave

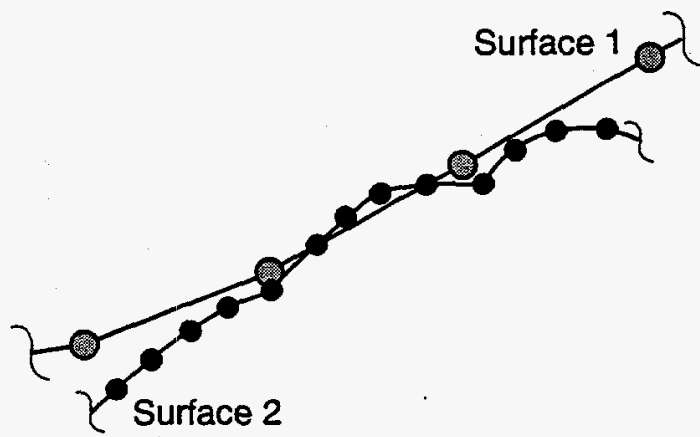

(b) Surface 1 is the slave and surface 2 is the master

Figure 5.1. Schematic showing the effect of changing the master-slave designation between two surfaces.

would be to check every slave node against every master surface at every time step. This, however, is inefficient. The current strategy is to use a global search to determine which slave nodes are in close proximity to a master surface. The search process accumulates these potential interactions by constructing a local neighborhood around the master surface and globally searching for all slave nodes that fall within the neighborhood. The algorithm is based on a particle search technique, Heinstein et al., 1993. It sorts the slave nodes by location and uses a binary search to construct a list of slave nodes in a master surface neighborhood. The search algorithm depends only on the number of slave nodes and not on the geometry of the problem. It takes advantage of the known positions of the slave nodes and master surfaces.

After gathering a list of potential interactions, a detailed contact check is done for each slave-node/master surface pair. This check determines: (1) which of the candidate master surfaces is in contact with the slave node, (2) the point of contact, (3) the amount of penetration, and (4) the direction the slave node should be pushed back. The contact check also tries to resolve pushback ambiguities that arise due to discretization of the master surface. A complete discussion of these ambiguities and their resolution can be found in Heinstein et al., 1993.

The benefits of reducing the time spent in the contact surface algorithms can be significant. For iterative solvers, such as dynamic relaxation, inaccuracies in the location phase lead to an increase in the number of iterations required for convergence. These inaccuracies arise mainly from incorrectly determining the location of contact as a slave node slides across another surface. For large finite element simulations with large numbers of slave nodes and master surfaces, as much as $50 \%$ of the total CPU time is spent in the location phase. Thus, the speed and efficiency of the contact detection algorithms are important. 


\subsection{Application Phase}

The operation of the master-slave scheme can be demonstrated in the following example where slave node I lies between master nodes $M$ and $N$, as shown in Figure 5.2.1. The location phase algorithm has already determined that node $I$ has penetrated the master surface segment connecting nodes $M$ and $N$. Because node $I$ has penetrated the master surface, the normal force $\left(R_{n}\right)$ and tangential force $\left(R_{t}\right)$ at node $I$ are determined. The coefficient of friction, $\mu$, is used in conjunction with $R_{n}$ to determine the threshold value for slip, $\mu R_{n}$. If $R_{t}$ is less than $\mu R_{n}$, then no slip occurs and both the values of $R_{n}$ and $R_{t}$ are transferred to the master surface nodes using a weighting procedure based on the position of the slave node along the master segment.

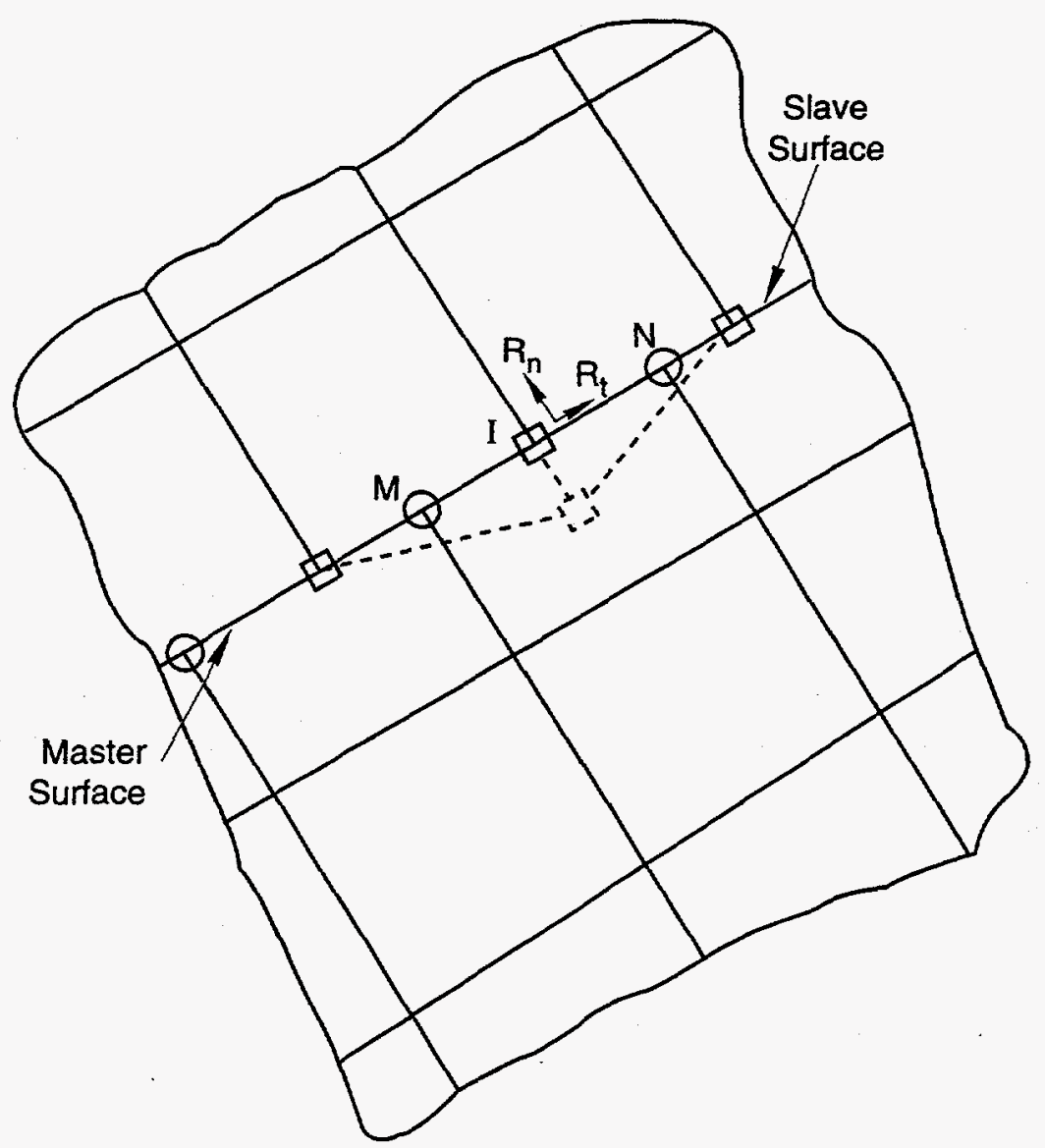

TRI-6348-21-0

Figure 5.2.1. Schematic showing the penetration of the master surface by slave node I. Node I is kinematically restored to the master surface, and its nodal forces are transferred to the master surface nodes $M$ and $\mathrm{N}$. 
The enforcement of the contact constraint is done using nodal velocities rather than displacements. In addition, the constraint is not enforced in one iteration but over several iterations to improve the convergence of the nonlinear equilibrium iterations. The nodal velocity of slave node $I$ is rotated into normal and tangential components. For the no-slip case, the tangential velocity of the slave node is assigned a new nodal velocity based on the tangential velocity of the corresponding point on the master surface. The normal velocity component of the slave node is given the normal velocity of the master surface modified by an incremental velocity based on the depth of penetration, $\delta$, by the slave node. The incremental velocity is applied in a direction to move the slave node back towards the master surface. The resulting equation for the normal velocity component is computed as

$$
v_{n}^{s}=v_{n}^{m}+0.1 \frac{\delta}{\Delta t}
$$

The pushback factor of 0.1 ensures that the slave node is not pushed back to the master surface in a single iteration. For the case when $R_{t}$ is greater than $\mu R_{n}$, slip can occur. The tangential force $R_{t}$ is reduced to its maximum allowable value of $\mu R_{n}$ which results in a force imbalance and allows movement of the slave node along the master surface to a new equilibrium position. The slave node forces, $R_{n}$ and $R_{t}$, are again transferred to the master nodes. The normal velocity of the slave node is modified exactly as in the no-slip case while the tangential velocity component is not modified at all.

The nodal mass associated with each contacting slave node is transferred to the appropriate master surface nodes using the weighting procedure used for transferring slave node forces. The transfer of mass is used by the solution algorithm during the calculation of the nodal accelerations, $a=\frac{F}{m}$. This transfer of mass, which is performed for each iteration, allows the master surface nodal accelerations to reflect the presence of the slave surface.

Incorporated within the contact surface model is the capability for two surfaces initially in contact to separate at a prescribed load level and for two distinct surfaces to contact and remain in contact during deformation. The user is allowed to specify the separation force level and to specify the separation tolerance with which both surfaces are assumed to be in contact. The default value of the separation force level is $1 . \times 10^{40}$. The default value of the separation tolerance is .02 times the length of the master surface segment. Inclusion of friction does have the effect of increasing the number of iterations required for convergence in many cases. 


\subsection{LOADS AND BOUNDARY CONDITIONS}

SANTOS contains several types of loads and boundary conditions. Displacements, pressures, concentrated forces, and body forces may be prescribed. In this section, we describe how these are implemented in the program.

\section{6:1 Kinematic Boundary Conditions}

The kinematic boundary conditions described below are all accomplished by altering the acceleration, velocities, and displacements of the nodal points. The application of these boundary conditions does not vectorize because they require a function look-up and a scatter of values. All of the kinematic boundary conditions are applied to nodal point sets.

\subsubsection{No-Displacement Boundary Conditions}

The no-displacement boundary conditions are accomplished by setting the appropriate component of the acceleration, velocity, and displacement of the node to zero. The imbalance force component for the node is accumulated with other no-displacement nodes to produce a total reaction force which is written as a global variable ( $\mathrm{RX}$ and RY) at each load step. The imbalance force component for the node is then set to zero.

\subsubsection{Prescribed Displacement Boundary Conditions}

The prescribed displacement boundary conditions are accomplished by setting the displacement component of the node point to the required displacement value corresponding to the end of the step. The appropriate components of the acceleration and velocity of the nodal points are set to zero along with the imbalance force component.

\subsubsection{Sloping Roller Boundary Conditions}

This displacement boundary condition requires the nodal point set to displace along a line defined by the analyst. The analyst defines the line by providing the components of the surface outward normal. The acceleration and velocity of the node point are rotated into a coordinate system normal and tangential to the line. The normal acceleration and velocity components are set to zero, and the remaining tangential components are rotated back to the global coordinate system. The imbalance force associated with the node is also rotated into the normal and tangential coordinate system. The normal force component is set to zero, and the remaining tangential force is rotated back into the global coordinate system.

\subsection{Traction Boundary Conditions and Distributed Loads}

The boundary conditions described below apply external forces to selected nodes. The pressure boundary condition is associated with element side sets while the nodal force boundary condition applies to nodal point sets. Body forces (distributed loads) are applied to each node in proportion to the mass of the material that surrounds it. 


\subsubsection{Pressure}

The set of consistent nodal point forces arising from pressures distributed over an element side is defined via the principle of virtual work by

$$
\delta u_{i I} f_{i I}=\delta u_{i I} \int_{s} \phi_{I}\left(-p n_{i}\right) d A
$$

where the range of the lower-case subscripts is 1 to 2 and the upper-case subscripts 1 to 4 .

Since the virtual displacements are arbitrary, they may be eliminated to yield

$$
\mathrm{f}_{\mathrm{iI}}=-\int_{s} \phi_{\mathrm{I}} \mathrm{pn}_{\mathrm{i}} \mathrm{dA}
$$

The most general pressure distribution we allow is mapped from nodal point pressure values via the isoparametric shape functions. The resulting expression for the consistent nodal forces is

$$
\mathrm{f}_{\mathrm{iI}}=-\mathrm{p}_{\mathrm{J}} \int_{\mathrm{s}} \phi_{\mathrm{I}} \phi_{\mathrm{J}} \mathrm{n}_{\mathrm{J}} \mathrm{dA}
$$

For the four-node constant stress element used in PRONTO, $\phi_{\mathrm{I}}$ is given by

$$
\phi_{\mathrm{I}}=\frac{1}{2} \Sigma_{\mathrm{I}}+\xi \Lambda_{\mathrm{I}}, \quad-\frac{1}{2} \leq \xi \leq \frac{1}{2}
$$

where

$$
\Sigma_{i}=\left\{\begin{array}{l}
1 \\
1
\end{array}\right\} \quad \Lambda_{I}=\left\{\begin{array}{c}
-1 \\
1
\end{array}\right\}
$$

and $\mathrm{n}_{\mathrm{i}} \mathrm{n}_{\mathrm{i}}=1$. For the geometry and pressure distribution shown in Figure 6.2.1, it can be shown that

$$
\mathrm{x}_{\mathrm{i}}=\mathrm{x}_{\mathrm{iI}} \phi_{\mathrm{I}}
$$




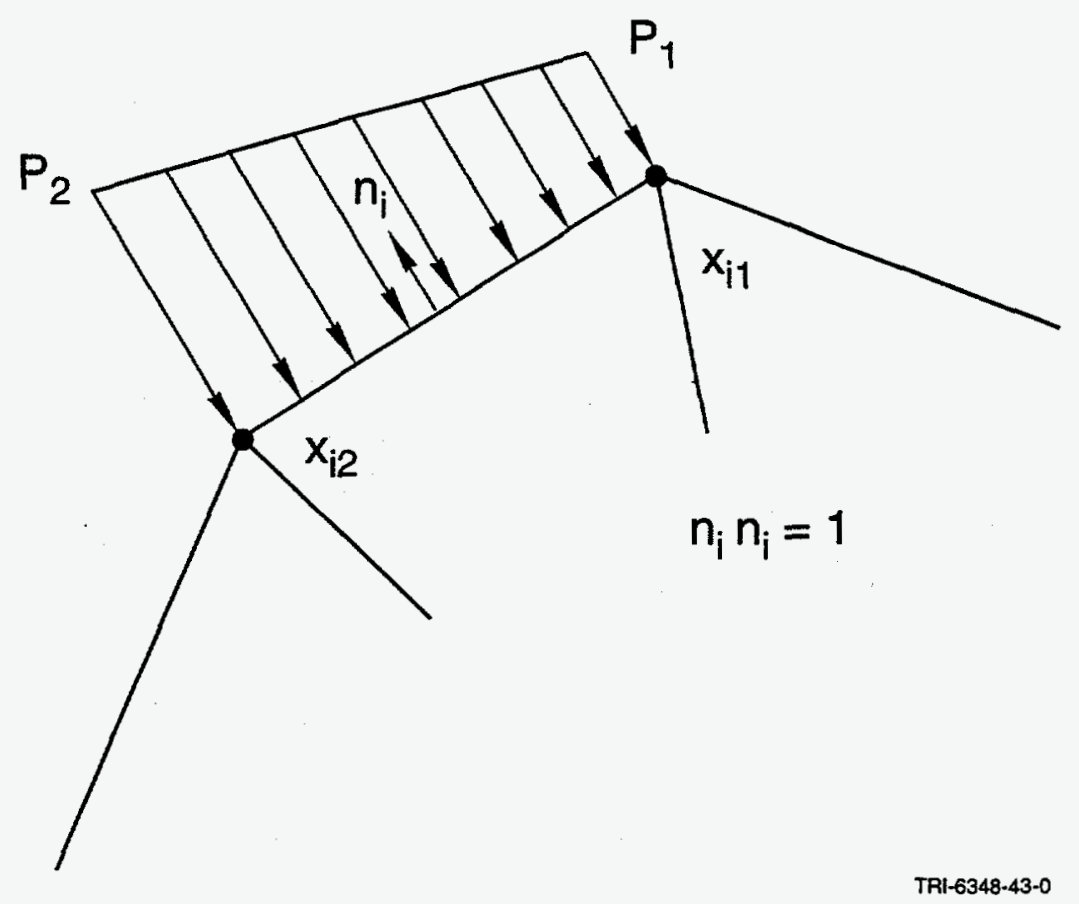

Figure 6.2.1. Definition of a pressure boundary condition along an element side.

and

$$
n_{i} d A=e_{i j 3} \frac{\partial x_{i}}{\partial \varepsilon_{j}} d \xi=e_{i j 3} x_{j K} \Lambda_{K} d \xi
$$

Then the consistent nodal forces can be written as

$$
f_{i I}=-p_{J} e_{i j 3} x_{j K} \Lambda_{K} \int_{-\frac{1}{2}}^{\frac{1}{2}} \phi_{I} \phi_{J} d \xi
$$

Combining Equations (6.2.4), (6.2.5), and (6.2.8):

$$
\mathrm{f}_{\mathrm{iI}}=-\mathrm{p}_{\mathrm{J}} \mathrm{e}_{\mathrm{ij} 3} \mathrm{x}_{\mathrm{jK}} \Lambda_{\mathrm{K}}\left[\frac{1}{4} \Sigma_{\mathrm{I}} \Sigma_{\mathrm{J}}+\frac{1}{12} \Lambda_{\mathrm{I}} \Lambda_{\mathrm{J}}\right] .
$$

The above expression is evaluated as

$$
N_{i}=-e_{i j 3} x_{j K} \Lambda_{K}=\left\{\begin{array}{l}
y_{1}-y_{2} \\
x_{2}-x_{1}
\end{array}\right\}
$$


and

$$
\mathrm{f}_{\mathrm{iI}}=\mathrm{N}_{\mathrm{i}} \cdot \frac{1}{6}\left[\begin{array}{ll}
2 & 1 \\
1 & 2
\end{array}\right] \cdot\left\{\begin{array}{l}
\mathrm{p}_{1} \\
\mathrm{p}_{2}
\end{array}\right\}
$$

The nodal values for the pressure are calculated using the user-supplied scale factor and time history function. The values are calculated for the beginning of the time step.

The application of the pressure boundary conditions is fully vectorized. Blocks of element sides are processed in vector blocks using the scratch element space. After the consistent nodal point forces are calculated for a block of element sides, they are accumulated into the global nodal force array.

\subsubsection{Adaptive Pressure}

The adaptive pressure boundary condition in SANTOS allows the analyst to model the behavior of gas-filled cavities that change internal pressure as the cavity deforms. The analyst defines the cavity using a side set identifier, and the volume of the cavity is computed every iteration based on the current deformed shape. The cavity volume is passed to SUBROUTINE FPRES, which is user supplied, where the gas pressure is computed. This gas pressure is then applied to the defined side set as a pressure boundary condition.

\subsubsection{Nodal Forces}

Nodal point external forces are applied to each point in the node set. The magnitude of the force is determined by the user-supplied scale factor and a time history function. The time history function is evaluated at the end of the load step. If the analysis type is axisymmetric, then the nodal forces are input as force per radian.

\subsubsection{Gravity Forces, Body Forces, and Distributed Loads}

Gravity and body forces are computed using the third integral in Equation (3.1.8). The same routines that compute the diagonal mass matrix are used to form the gravity load vector. The density, $\rho$, is the input density for the specific material and not the fictitious density computer for the stable time increment. The component direction accelerations are specified on the GRAVITY input card. In addition to the $x$ - and $y$-direction accelerations, the code supports an angular acceleration. The magnitude of the acceleration is determined by the user-supplied scale factor and a time history function. The time history function is evaluated at the end of the load step.

The nodal values of the distributed loads are read from an externally written file, fort.38, in the following format:

$$
\text { READ(38) TIME, (DISTX(I), I=1,NNOD), (DISTY(I), I=1,NNOD) }
$$

at the desired time intervals. DISTX and DISTY are the nodal point distributed load components in the $\mathrm{x}$ and $\mathrm{y}$ directions, respectively. These nodal values are in units of force per unit volume. This force/volume is multiplied by the appropriate nodal volume to obtain the magnitude of the nodal loading. The nodal volume is computed using the 
diagonal mass matrix routines with a density equal to one. An example of this type of loading is the body force generated by the presence of a magnetic field.

\subsubsection{Thermal Forces}

Nodal point temperatures for performing thermal/structural analyses are input into SANTOS in two ways. The first method is to read an externally written temperature file, fort.56, in the following format:

$$
\text { READ(56) TIME, (T(I), I = 1,NNOD) }
$$

where the temperature, $T$, is read for each nodal point. SANTOS interpolates linearly between thermal time steps to obtain the thermal solution for the time requested by the structural analysis. On the THERMAL STRESS input card, the entry type is set to EXTERNAL to request this method.

The second method is best suited for problems where the structure is heated uniformly. The analyst can define the temperature history of the body using an input function, and SANTOS will interpolate the body temperature at the time requested by the structural analysis. On the THERMAL STRESS input card, the entry type is set to INTERNAL.

SANTOS requires the analyst to input, for each material, the curve of thermal strain versus temperature. This curve is defined using an input function. With the temperature of each element known, it is a simple process to interpolate the thermal strain for each element. If we difference the thermal strain computed at the beginning and at the end of the load/time step and divide by $\Delta t$, we obtain a thermal strain rate. SANTOS computes the total strain rate, using the velocity gradients, which can be decomposed into mechanical and thermal strain rate components. The thermal strain rate is subtracted from the total strain rate to obtain the mechanical strain rate, which is passed into the SANTOS constitutive models. 
Intentionally Left Blank 


\subsection{REFERENCES}

Biffle, J.H., and M.L. Blanford. 1994. JAC2D - A Two-Dimensional Finite Element Computer Program for the Nonlinear Quasi-Static Response of Solids with the Conjugate Gradient Method. SAND93-1891. Albuquerque, NM: Sandia National Laboratories.

Cormeau, I. 1975. "Numerical Stability in Quasi-Static Elasto/Visco-Plasticity," International Journal for Numerical Methods in Engineering. Vol. 9, no. 1, 109-127.

Costin, L.S., and C.M. Stone. 1985. A Linear Viscoelastic Material Mode for SANCHO. SAND85-1836. Albuquerque, NM: Sandia National Laboratories.

Dienes, J.K. 1979. "On the Analysis of Rotation and Stress Rate in Deforming Bodies," Acta Mechanica. Vol. 32, 217-232.

Ferry, J.D. 1950. "Mechanical Properties of Substances of High Molecular Weight. VI. Dispersion in Concentrated Polymer Solutions and its Dependence on Temperature and Concentration," Journal of the American Chemical Society. Vol. 72, 3746-3752.

Ferry, J.D. 1970. Viscoelastic Properties of Polymers. 2nd ed. New York, NY: John Wiley \& Sons, Inc.

Flanagan, D.P., and T. Belytschko. 1981. "A Uniform Strain Hexahedron and Quadrilateral with Orthogonal Hourglass Control," International Journal for Numerical Methods in Engineering. Vol. 17, 679-706.

Flanagan, D.P., and T. Belytschko. 1984. "Eigenvalues and Stable Time Steps for the Uniform Strain Hexahedron and Quadrilateral," Transactions of the ASME, Journal of Applied Mechanics. Vol. 51, no. 1, 35-40.

Flanagan, D.P., and L.M. Taylor. 1987. "An Accurate Numerical Algorithm for Stress Integration with Finite Rotations," Computer Methods in Applied Mechanics and Engineering. Vol. 62, 305-320.

Goodman, R.E., and J. Dubois. 1972. "Duplication of Dilitancy in Analysis of Jointed Rocks," Journal of the Soil Mechanics and Foundations Division, Proceedings of the American Society of Civil Engineers. Vol. 98, no. 4, 399-422.

Goudreau, G.L., and J.O. Hallquist. 1982. "Recent Developments in Large-Scale Finite Element Lagrangian Hydrocode Technology," Computer Methods in Applied Mechanics and Engineering. Vol. 33, no. 1-3, 725757.

Heinstein, M.W., S.W. Attaway, J.W. Swegle, and F.J. Mello. 1993. A General-Purpose Contact Detection Algorithm for Nonlinear Structural Analysis Codes. SAND92-2141. Albuquerque, NM: Sandia National Laboratories.

Hibbitt, Karlsson \& Sorensen, Inc. 1992. ABAQUS, Theory Manual, Version 5.2. Pawtucket, RI: Hibbitt, Karlsson $\&$ Sorensen, Inc. (Copy of file in the Sandia WIPP Central Files, Sandia National Laboratories, Albuquerque, NM as WP036332.)

Holcomb, D.J., and M. Shields. 1987. Hydrostatic Creep Consolidation of Crushed Salt with Added Water. SAND87-1990. Albuquerque, NM: Sandia National Laboratories.

Hughes, T.J.R. 1987. The Finite Element Method: Linear Static and Dynamic Finite Element Analysis. Englewood Cliffs, NJ: Prentice-Hall. 
Hughes, T.J.R., and J. Winget. 1980. "Finite Rotation Effects in Numerical Integration of Rate Constitutive Equations Arising in Large-Deformation Analysis," International Journal for Numerical Methods in Engineering. Vol. 15, no. 12, 1862-1867.

Johnson, G.C., and D.J. Bammann. 1984. "A Discussion of Stress Rates in Finite Deformation Problems," International Journal of Solids and Structures. Vol. 20, no. 8, 725-737.

Key, S.W., Z.E. Beisinger, and R.D. Krieg. 1978. HONDO II - A Finite Element Computer Program for the Large Deformation Dynamics Response of Axisymmetric Solids. SAND78-0422. Albuquerque, NM: Sandia National Laboratories.

Krieg, R.D. 1978. A Simple Constitutive Description for Cellular Concrete. SC-DR-72-0883. Albuquerque, NM: Sandia National Laboratories. (Copy on file in the Sandia WIPP Central Files, Sandia National Laboratories, Albuquerque, NM as WP036331.)

Krieg, R.D., and D.B. Krieg. 1977. "Accuracies of Numerical Solution Methods for the Elastic-Perfectly Plastic Model," ASME Journal of Pressure Vessel Technology. Vol. 99, 510-515.

Leaderman, H. 1943. Elastic and Creep Properties of Filamentous Materials and Other High Polymers. Washington, DC: Textile Foundation, Inc.

Morland, L.W., and E.H. Lee. 1960. "Stress Analysis for Linear Viscoelastic Materials with Temperature Variation," Transactions of the Society of Rheology. Vol. IV, 233-263.

Muki, R., and E. Sternberg. 1961. "On Transient Thermal Stresses in Viscoelastic Materials with TemperatureDependent Properties," Journal of Applied Mechanics. Vol. 28, Series E, no. 2, 193-207.

Munson, D.E., and P.R. Dawson. 1979. Constitutive Model for the Low Temperature Creep of Salt (with Application to WIPP). SAND79-1853. Albuquerque, NM: Sandia National Laboratories.

Munson, D.E., and P.R. Dawson. 1982. A Transient Creep Model for Salt During Stress Loading and Unloading. SAND82-0962. Albuquerque, NM: Sandia National Laboratories.

Munson, D.E., and P.R. Dawson. 1984. "Salt Constitutive Modeling Using Mechanism Maps," The Mechanical Behavior of Salt, Proceedings of the First Conference, Pennsylvania State University, University Park, PA, November 9-11, 1981. Eds. H.R. Hardy, Jr. and M. Langer. Rockport, MA: Karl Distributors. 717-737.

Munson, D.E., A.F. Fossum, and P.E. Senseny. 1988. Advances in Resolution of Discrepancies Between Predicted and Measured In Situ WIPP Room Closures. SAND88-2948. Albuquerque, NM: Sandia National Laboratories.

Neilsen, M.K., H.S. Morgan, and R.D. Krieg. 1987. A Phenomenological Constitutive Model for Low Density Polyurethane Foams. SAND86-2927. Albuquerque, NM: Sandia National Laboratories.

Otter, J.R.H., A.C. Cassell, and R.E. Hobbs. 1966. "Dynamic Relaxation," Proceedings of the Institution of Civil Engineers. Paper No. 6986. Vol. 35, 633-656.

Papadrakakis, M. 1981. "A Method for the Automatic Evaluation of the Dynamic Relaxation Parameters," Computer Methods in Applied Mechanics and Engineering. Vol. 25, 35-48.

Red-Horse, J.R., W.C. Mills-Curran, and D.P. Flanagan. 1990. SUPES Version 2.1 - A Software Utilities Package for the Engineering Sciences. SAND90-0247. Albuquerque, NM: Sandia National Laboratories. 
Schreyer, H.L., R.F. Kulak, and J.M. Kramer. 1979. "Accurate Numerical Solutions for Elastic-Plastic Models," ASME Journal of Pressure Vessel Technology. Vol. 101, 226-234.

Schwarzl, F., and A.J. Staverman. 1952. "Time-Temperature Dependence of Linear Viscoelastic Behavior," Journal of Applied Physics. Vol. 23, no. 8, 838-843.

Sjaardema, G.D. 1993. Overview of the Sandia National Laboratories Engineering Analysis Code Access System. SAND92-2292. Albuquerque, NM: Sandia National Laboratories.

Sjaardema, G.D., and R.D. Krieg. 1987. A Constitutive Model for the Consolidation of WIPP Crushed Salt and Its Use in Analyses of Backfilled Shaft and Drift Configurations. SAND87-1977. Albuquerque, NM: Sandia National Laboratories.

Stone, C.M., R.D. Krieg, and Z.E. Beisinger. 1985. SANCHO - A Finite Element Computer Program for the Quasistatic, Large Deformation, Inelastic Response of Two-Dimensional Solids. SAND84-2618. Albuquerque, NM: Sandia National Laboratories.

Stone, C.M., G.W. Wellman, and R.D. Krieg. 1990. A Vectorized Elastic/Plastic Power Law Hardening Material Model Including Luders Strain. SAND90-0153. Albuquerque, NM: Sandia National Laboratories.

Taylor, L.M., and D.P. Flanagan. 1987. PRONTO 2D - A Two-Dimensional Transient Solid Dynamics Program. SAND86-0594. Albuquerque, NM: Sandia National Laboratories.

Taylor, L.M., and D.P. Flanagan. 1988. An External Code Interface for the PRONTO Family of Transient Solid Dynamics Programs. SAND87-3003. Albuquerque, NM: Sandia National Laboratories.

Truesdell, C.A. 1966. The Elements of Continuum Mechanics. New York, NY: Springer-Verlag.

Underwood, P.G. 1983. "Dynamic Relaxation," Computational Methods for Transient Analysis. Eds. T. Belytschko and T.J.R. Hughes. New York, NY: Elsevier. 245-265.

Williams, M.L. 1955. "The Temperature Dependence of Mechanical and Electrical Relaxations in Polymers," Journal of Physical Chemistry. Vol. 59, 95-96.

Williams, M.L., R.F. Landel, and J.D. Ferry. 1955. "The Temperature Dependence of Relaxation Mechanisms in Amorphous Polymers and Other Glass-forming Liquids," Journal of the American Chemical Society. Vol. 77, 3701-3707. 
Intentionally Left Blank 
APPENDIX A:

SANTOS Users Manual 
Intentionally Left Blank

A-2 


\section{SANTOS Users Manual}

Listed below, in the order they appear in the text, are all the key words used in the SANTOS input.

1. TITle

2. PLAne STRain

3. AXIsymmetric

4. STEP CONtrol

5. AUTo STEp

6. OUTput TIme

7. PLOT TIme

8. PLOT NODal

9. PLOT ELement

10. PLOT STate

11. TIme STep SCale

12. RESidual TOLerance

13. MAXimum ITerations

14. INTermediate PRint

15. MAXimum TOLerance

16. GLObal CONvergence

17. LOCal CONvergence

18. ELAStic SOLution

19. PREdictor SCAle FACtor

20. DISTributed LOAds

21. INITial STRess

22. THERmal STRess

23. GRAVity

24. MINimum DAMping FACtor

25. NO DAMping

26. WRITe REStart

27. READ REStart

28. HOURglass STIFfening

29. XBEGIN

30. XEND

31. EXIT

32. FUNCtion

33. NO DISplacement

34. PREScribed DISPlacement

35. SLOping ROLler

36. PREScribed FORce

37. PRESsure

38. ADAptive PREssure

39. CONtact SURface

40. RIGid SURface

41. MATerial

42. MATerial POint 


\section{DELete MATerial}

The input data to SANTOS is in a free field form using key words. The key words are intended to define a user friendly program language input. The input is order independent and can be entered in any order the user finds convenient. The words as typed below in UPPER CASE represent keywords in the list above. Most of the words can be abbreviated to the first few characters. In the list above the upper case characters indicate the shortest abbreviation allowed. The words typed in lower case below indicate variables for which the user should enter a value. An example data file is shown below.

The free field input allows the user to delineate entries by either a blank, a comma, or an equals sign. We find it useful to use blanks with commands (keywords), equal signs to separate keywords and/or lists, and commas for lists of values. The material data requires material cues and their associated values and equal signs are useful there. See the example input below.

A dollar sign indicates that whatever follows on the line of input is a comment and is ignored. An asterisk indicates that the line is to be continued on the next line.

\section{TITLE}

(enter a suitable title on the next line)

\section{PLANE STRAIN}

Indicates that a plane strain analysis is to be performed. If the analysis type keyword is omitted then a plane strain analysis is selected as default.

\section{AXISYMMETRIC}

Indicates that an axisymmetric analysis is to be performed.

\section{STEP CONTROL}

$$
\begin{gathered}
\mathbf{n}, \mathbf{t 1} \\
\mathbf{m}, \mathbf{t 2} \\
., \cdot \\
\text { END }
\end{gathered}
$$

$n$... number of load steps in interval $0<t<t 1$

$t 1$... end time for first load step interval

$m$... number of load steps in interval $\mathrm{t} 1<\mathrm{t}<\mathrm{t} 2$

t2 ... end time for second load step interval

This command specifies the static load control parameters for the problem. The analysis is assumed to begin at $t=0$ and take $n$ steps to time $t 1$. The code then will take $m$ load steps to time t2. This set of input is completed by an END card. Any number of step-time cards is allowed.

\section{AUTO STEP, tol, dtmax, dtmin, dtinit}


tol ... tolerance value used for controlling change of time step in material model

$d t m a x$... maximum value of time step allowed. Values of $\mathrm{dt}$ computed to be greater than $\mathrm{dtmax}$ will be set to $\mathrm{dtmax}$.

dtmin ... minimum value of time step allowed. Computed time step cannot be smaller then this value. If the user does not wish the time step to shrink insert NOREDUCE in this field.

dtinit ... initial value of time step to be used in the calculation

This command can be used to automatically grow the solution time step for any time dependent material model that allows such a feature. The command specifies a solution tolerance change allowed over the step, along with allowable values of the time step (dtmax, dtmin, dtinit). The time step will grow and shrink according to satisfaction of tol. If the user does not wish to allow the time step to shrink, then insert the word NOREDUCE in place of dtmin.

\section{OUTPUT TIME}

$$
\begin{gathered}
\mathbf{n , t 1} \\
\mathbf{m}, \mathbf{t 2} \\
., . \\
\ddot{\text { END }}
\end{gathered}
$$

$n$... frequency of printed output in interval $0<\mathrm{t}<\mathrm{t} 1$

$t 1$... end time for first output time control

$m$... frequency of printed output in interval $\mathrm{t} 1<\mathrm{t}<\mathrm{t} 2$

$t 2$... end time for second output time control

This command specifies how often the requested printed output is to be written to the output file. The required information is an integer number specifying how often, not the number of outputs, the printed information is to be written. For example, if $\mathrm{n}$ or $\mathrm{m}$ is 1 then the output file will be written every load increment. If $n$ or $m$ is 2 then the file will be written every 2 load increments. Currently, the times $\mathrm{t} l$ and $\mathrm{t} 2$ must match the values specified on the STEP CONTROL card. An END card terminates this section of input.

\section{PLOT TIME}

$$
\begin{gathered}
\mathbf{n , t 1} \\
\mathbf{m}, \mathbf{t 2} \\
\cdot, \cdot \\
\text { END } \\
\text {,. }
\end{gathered}
$$

$$
\begin{aligned}
& n \ldots \text { frequency of plotted output in interval } 0<\mathrm{t}<\mathrm{t} 1 \\
& t 1 \ldots \text {. end time for first output time control } \\
& m \ldots \text { frequency of plotted output in interval } \mathrm{t} 1<\mathrm{t}<\mathrm{t} 2 \\
& t 2 \ldots \text { end time for second output time control }
\end{aligned}
$$

This command specifies how often the requested plotting output is to be written to the output file. 
The required information is an integer number specifying how often, not the number of outputs, the plotting information is to be written. For example, if $\mathrm{n}$ or $\mathrm{m}$ is 1 then the output file will be written every load increment. If $\mathbf{n}$ or $\mathrm{m}$ is 2 then the file will be written every 2 load increments. Currently, the times $t 1$ and $t 2$ must match the values specified on the STEP CONTROL card. An END card terminates this section of input.

\section{PLOT NODAL, nodal name 1 , nodal name $2, \ldots .$. allowable nodal variable names: \\ DISPLACEMENT - nodal displacements (DISPLX,DISPLY) \\ RESIDUAL - nodal residuals (RESIDX, RESIDY) and a scalar value (RESID) \\ MASS - nodal lumped masses (MASS) \\ REACTION - nodal force reactions (FX, FY)}

The default nodal variables written on the plotting data base are the displacements whether requested or not. The MASS specification results in having the lumped nodal masses written on the data base. The names in parenthesis indicate the alphanumeric name of the variables which are written on the plotting data base.

9. PLOT ELEMENT, element variable 1, element variable 2, .... allowable element variable names:

STRESS - stresses (SIGXX,SIGYY,SIGZZ,TAUXY)

STRAIN - total strains (EPSXX,EPSYY,EPSZZ,EPSXY)

RATEDFM - deformation rates (DXX,DYY,DZZ,DXY)

STRETCH - material stretches: V of F = V R (STRECHXX,STRECHYY,STRECHZZ,STRECHXY)

ROTATION - material rotations: $R$ of $F=V R$ (COSTHETA,SINTHETA)

DENSITY - current mass per unit volume (DENSITY)

PRESSURE - pressures (PRESSURE)

VONMISES - Von Mises equivalent stress (VONMISES)

HG - hourglass resistance forces (HGX,HGY)

EFFMOD - element effective modulus values used for the stable time step and mass scaling (EFFMOD)

TEMPERATURE - element centroidal temperatures (TEMP)

The names in parenthesis indicate the alphanumeric name of the variables which are written on the plotting data base. The default element variables are the stresses.

10. PLOT STATE, state variable 1, state variable $2, \ldots .$.

The user can ask for any of the internal state variables to be written on the plotting data base. Since all materials do not have the same internal state variables (some have none), a zero will be written on the data base for an element using a material model that does not have a state variable which is specified by the user. Hence, if the user asks for EQPS (equivalent plastic strain) and ALPHA11,ALHA22,ALPHA33, and ALPHA12 (back stress components for kinematic hardening) and he has a model where half the mesh uses the ELASTIC material and half the mesh uses the ELASTIC PLASTIC material, much of the data written on the plotting data base will contain zeros. The table below gives all the internal state variables names for all the material models. 
See the theory section for definitions of the variables if they are not obvious. The default state variables are none. WARNING: Indiscriminate use of this option can create extremely large plotting data bases.

allowable state variable names:

ELASTIC $=$ (no internal state variables)

ELASTIC PLASTIC = EQPS ALPHA11 ALPHA22 ALPHA33 ALPHA12 RADIUS

POWER LAW CREEP = EQCS

LOW DEN FOAM = (no internal state variables)

SOIL N FOAMS = EVMAX EVFRAC EV NUM

EP POWER HARD = RADIUS EQPS

LINEAR VISCOELASTIC = BLKDECAY DECAYX1 DECAYY1 DECAYZ1 DECAYXY1

DECAYX2 DECAYY2 DECAYZ2 DECAYXY2 DECAYX3 DECAYY3 DECAYZ3

DECAYXY3

THERMO EP = EQPS YM0 YM1 XNU0 XNU1 YS0 YS1 RADIUS

THERMOELASTIC = YM0 YM1 XNU0 XNU1

VOLUMETRIC CREEP = EQCS DENSITY

M-D CREEP MODEL = EQCS ZETA SDOT TRESCA ETSTAR

11. TIME STEP SCALE, scft

$s c f t$... scale factor to be applied to the internally calculated time step (default $=1.0$ )

12. RESIDUAL TOLERANCE, value

value ... number, in percent, that is used to check for equilibrium and convergence of the solution. Default is 0.5 .

13. MAXIMUM ITERATIONS, value

value ... number of iterations allowed for any solution step. Default is two times the number of nodes.

14. INTERMEDIATE PRINT, value

value ... frequency of intermediate print that provides information such as current equilibrium imbalance, number of steps, and applied load magnitudes.

15. MAXIMUM TOLERANCE, value

value ... when the maximum number of iterations is reached, if the convergence tolerance is less than value then the solution is assumed to be converged and the problem is advanced to the next solution step.

\section{GLOBAL CONVERGENCE}

This card specifies that a global convergence measure is to be used for determining satisfaction of equilibrium. This is the default method.

17. LOCAL CONVERGENCE, plocal 
plocal ... threshold residual value to be used in computation of local convergence. Values of the nodal residual below this value will be replaced by plocal in the convergence check. The default is plocal $=1.0$.

This card specifies that a local convergence measure will be used for determining satisfaction of equilibrium. The convergence check is done on a node by node basis and convergence is assumed if each nodal residual is below the specified value on the RESIDUAL TOLERANCE card.

\section{ELASTIC SOLUTION}

This specifies that a load step is requested using only time independent material response for the step. This card should be used only with the time-dependent material models.

\section{PREDICTOR SCALE FACTOR, function id}

function id... function id controlling the definition of the predictor scale factor

This option specifies the function id which defines the multiplier to be used for predicting the displacements on the next load step. The multiplier is used on the incremental changes in displacement over the previous load increment. The multiplier can be useful for reducing the number of iterations required for a solution. The default value of the multiplier is 1 .

\section{DISTRIBUTED LOADS}

This option specifies that an external file 38 is to be read for nodal values of a distributed force per unit volume. This force/volume is multiplied by the nodal volume to obtain the magnitude of the required loading. An example of this option is the body force generated by the presence of a magnetic field.

\section{INITIAL STRESS, type, sig1, sig2, sig3, sig4}

type ... specifies how the initial stress state will be specified. The choices are 'USER', or 'CONSTANT'. If 'USER' is selected then a user written subroutine must be supplied. If type is 'CONSTANT' then the values of sig1, sig2, sig3, and sig4 must be provided. The stresses will be assigned to each element in the model.

22. THERMAL STRESS, type, to, ithf, thforc

type ... identifies that a thermal stress analysis is to be performed. Default is no thermal stress analysis. If type is 'EXTERNAL' then an external file56 is required in the proper format for use by the code. If type is 'INTERNAL' then the following input parameters are needed.

to ... initial stress free temperature. Default is 0 .

ithf ... function id controlling temperature time response

thforc ... thermal load norm for use in convergence tests. This parameter will be used as the applied load norm. The default is 0 .

\section{GRAVITY, igrvf, gravx, gravy, omega}

igrvf ... function id controlling load time response

gravx ... specified acceleration in $\mathrm{x}$-direction

gravy ... specified acceleration in $\mathrm{y}$-direction 
omega ... specified angular velocity

\section{MINIMUM DAMPING FACTOR, fac}

fac ... this option allows the user to define the minimum allowable damping factor used in the dynamic relaxation algorithm. The allowable values of damping range from zero to one. If a damping value is computed that is less than fac, then the value is set equal to fac. The default value is set to 0.2 .

\section{NO DAMPING, iter, ndstep}

iter ... number of iterations with zero damping.

ndstep ...number of load steps with zero damping

This option is useful for problems with thin beam like behavior. The problem is allowed to deform without damping for a user specified number of iterations. This allows the problem to more quickly reach the fundamental deformation mode before damping begins. Iter should be selected as twice the number of elements meshed along the length direction. The normal damping algorithm can be initiated after performing ndstep load steps.

\section{WRITE RESTART, $n$}

$n$... this option specifies that a SANTOS restart tape is to be written at a frequency of every $\mathbf{n}$ increments. This information is written to file 30 .

\section{READ RESTART, $\mathbf{n}$}

$n$... this option specifies that a SANTOS restart tape is to be read at step $n$ and a new analysis performed. Some internal checking is performed to insure that the restart tape is valid. The restart tape is assigned to file 32 .

28. HOURGLASS STIFFENING, hgstiff, hgvis

hgstiff ... hourglass stiffening factor (default $=.05$ for plane strain and .01 for axisymmetric)

hgvis ... hourglass viscosity factor (default $=.0$ for plane strain and .03 for axisymmetric)

\section{XBEGIN, code name}

code name ... name of the external code to be coupled with SANTOS.

This option indicates that the following lines are input data for the external code

\section{XEND}

This option indicates an end to the external code input data

31. EXIT (required to terminate the input data)

\section{FUNCTION, function id}

function id ... any nonzero number you wish to identify with this function; after a FUNCTION statement you must enter a list of points defining your function:

$$
\begin{gathered}
x 1, f(x 1) \\
\times 2, f(x 2) \\
., .
\end{gathered}
$$




\section{$\mathbf{x n}, \mathbf{f}(\mathbf{x n})$ \\ END}

(The list is terminated by a line containing the word END as shown. Any other valid input cue will also work)

If the function represents a time history function to be used with one of the nodal boundary condition specifications (e.g. PRESCRIBED DISPLACEMENT) or with a PRESSURE boundary condition, if the value of time is not within the limits defined by $x 1$ and $x n$, no boundary condition will be applied until the current value of time falls within the limits. This means that you can have a boundary condition turn on at a specific time and/or turn off at a specific time.

\section{NO DISPLACEMENT, direction, node set fiag} direction ... either $\mathrm{X}$ or $\mathrm{Y}$

node set flag ... identifying number from the input data base which identifies the nodes you want to have no displacement (note: this is a nodal bc!)

34. PRESCRIBED DISPLACEMENT, dir, node set flag, function id, scale factor, a0, b0 dir ... either X, Y, RADIAL, TANGENT, or NORMAL

node set flag ... identifying number from the input data base which identifies the nodes you want to have this displacement (note: this is a nodal bc!)

function id ... identifying number of the function you want to use to specify the time dependence of the displacement

scale factor ... scale factor to be applied to the function (default=1.0)

$a 0, b 0 \ldots$ not used (if direction $=\mathrm{X}$ or $\mathrm{Y}$ ) center of cylinder of sphere (if direction = RADIAL or TANGENT) components of normal (if direction = NORMAL)

\section{SLOPING ROLLER, node set flag, $\mathrm{n} 1, \mathrm{n} 2$}

node set flag ... identifying number from the input data base which identifies the nodes that have this bc (note: this is a nodal bc!)

$n 1, n 2 \ldots$ components of the surface outward normal

36. PRESCRIBED FORCE, direction, node set flag, function id, scale factor, a0, b0 direction ... either X, Y, RADIAL, TANGENT, or NORMAL

node set flag ... identifying number from the input data base which identifies the nodes you want to have this force (note: this is a nodal bc!)

function id ... identifying number of the function you want to use to prescribe the time dependence of the force

scale factor ... scale factor which will be applied to the function (default=1.0)

$a 0, b 0 \ldots$ not used (if direction $=\mathrm{X}$ or $\mathrm{Y}$ ) center of cylinder of sphere (if direction = RADIAL or TANGENT) components of normal (if direction = NORMAL)

37. PRESSURE, side set flag, function id, scale factor

side set flag ... identifying number from the input data base which identifies the sides you want to have this pressure (note: this is a side or element bc!)

function id ... identifying number of the function you want to use to prescribe the time depen- 
dence of the pressure

scale factor ... scale factor which will be applied to the function (default=1.0)

38. ADAPTIVE PRESSURE, side set flag, $x 0, y 0$

side set flag ... identifying side set flag from the input data base which identifies the sides you want to have this pressure

$x 0, y 0 \ldots$ coordinates of point used to determine cavity area

This option allows the user to define a pressure boundary condition which depends on the solution. The user can write a subroutine FPRES which can adaptively apply a pressure boundary condition based on various factors. An example of this option is the application of pressure due to compression of an ideal gas.

39. CONTACT SURFACE, side set flag 1, side set flag 2 , mu,dis, tenrel side set flag 1 ... identifying number from the input data base which identifies the master surface. (note: this is a side or element bc!)

side set flag 2 ... identifying number from the input data base which identifies the slave surface. (note: this is a side or element bc!)

$m u$... coefficient of friction (default $=0$.) if $m u=$ FIXED then the surfaces are treated as fixed surfaces

dis ... fraction of element side length used to determine tolerance for proximity to master surface check (default is 1.e-8)

tenrel ... residual normal force acting on the slave node used to determine release conditions. $($ default $=1 . e 40)$

40. RIGID SURFACE, slave flag, $\mathrm{x0}, \mathrm{y0}, \mathrm{nx}, \mathrm{ny}$, mu

slave flag ... identifying number from the input data base which identifies sides that are slaved to the rigid surface (note: this is a side or element bc!)

$x 0, y 0 \ldots$ coordinates of a point on the rigid surface

$n x, n y \ldots$ outward unit normal to the rigid surface

$m u \ldots$ coefficient of friction (default $=0$.) If $m u=$ FIXED then the surface is assumed to be fixed to the rigid surface.

41. MATERIAL, material id flag, material name, density, func id, thermal strain scaling factor

material id ... material identification number from the input data base

material name ... valid material type name

The current material types allowed in SANTOS are:

ELASTIC

ELASTIC PLASTIC

POWER LAW CREEP

LOW DEN FOAM

SOIL N FOAMS

EP POWER HARD

LINEAR VISCOELASTIC

THERMO EP 
THERMOELASTIC

VOLUMETRIC CREEP

M-D CREEP MODEL

density ... material density

func id ... function id to be used for specifying function number with proper thermal strain variation with temperature. This value needed for THERMAL STRESS problems.

thermal strain ... scaling factor multiplier for function values given from thermal strain function. The default value of this factor is 1 .

The allowable material names and their required material cues are given below. The material data can be entered in any order separated by commas. An END statement is required to terminate the material data. The material constant associated with each material cue, as defined in Section 4 , is given in parentheses.

1. ELASTIC (number of cues=2)

YOUNGS MODULUS (E)

POISSONS RATIO $(v)$

2. ELASTIC PLASTIC (number of cues=5)

YOUNGS MODULUS (E)

POISSONS RATIO $(v)$

YIELD STRESS $\left(\sigma_{y s}\right)$

HARDENING MODULUS (H)

$\operatorname{BETA}(\beta)$

3. POWER LAW CREEP (number of cues=5)

TWO MU $(2 \mu)$

BULK MODULUS (K)

CREEP CONSTANT (A)

STRESS EXPONENT (m)

THERMAL CONSTANT ( $\frac{Q}{R \Theta}$ if isothermal or $\frac{Q}{R}$ if not)

4. LOW DEN FOAM (number of cues $=7$ )

YOUNGS MODULUS (E)

A

B

C

NAIR (contribution of trapped air to foam response; 1 for yes, 0 for no contribution)

PO

PHI $(\phi)$

5. SOIL N FOAMS (number of cues=7) 
TWO MU $(2 \mu)$

BULK MODULUS (K)

A0

A1

A2

FUNCTION ID (function number of curve defining pressure-volume strain relationship)

PRESSURE CUTOFF (valid (negative) tensile fracture pressure)

6. EP POWER HARD (number of cues=6)

YOUNGS MODULUS (E)

POISSONS RATIO $(v)$

YIELD STRESS $\left(\sigma_{y s}\right)$

HARDENING CONSTANT (A)

HARDENING EXPONENT $(\mathrm{m})$

LUDERS STRAIN $\left(\varepsilon_{L}\right)$

7. LINEAR VISCOELASTICITY (number of cues $=13$ )

BULK (K)

BULK INF $\left(K^{\infty}\right)$

BULK RELAX $\left(\beta^{K}\right)$

SHEAR INF $\left(G^{\infty}\right)$

SHEAR ONE $\left(G_{1}\right)$

$\operatorname{SHEAR}$ TWO $\left(G_{2}\right)$

SHEAR THREE $\left(G_{3}\right)$.

RELAX ONE $\left(\beta_{1}^{s}\right)$

$\operatorname{RELAX}$ TWO $\left(\beta_{2}^{s}\right)$

RELAX THREE $\left(\beta_{3}^{s}\right)$

$\mathrm{C} 1\left(C_{1}^{0}\right)$

$\mathrm{C} 2\left(C_{2}^{0}\right)$

TEMP0 (reference temperature for the material properties)

8. THERMO EP (number of cues $=9$ )

YOUNGS MODULUS (E)

POISSONS RATIO $(v)$

YIELD STRESS $\left(\sigma_{y s}\right)$

MODULUS FUNCTION (function defining Young's modulus variation with $\Theta$ )

PR FUNCTION (function defining Poisson's ratio variation with $\Theta$ )

YIELD FUNCTION (function defining yield stress variation with $\Theta$ )

HARDENING CONSTANT (A)

HARDENING EXPONENT (m) 


\section{LUDERS STRAIN $\left(\varepsilon_{L}\right)$}

9. THERMOELASTIC (number of cues =4)

YOUNGS MODULUS (E)

POISSONS RATIO $(v)$

MODULUS FUNCTION (function defining Young's modulus variation with $\Theta$ )

PR FUNCTION (function defining Poisson's ratio variation with $\Theta$ )

10. VOLUMETRIC CREEP (number of cues =12)

TWO MU $(2 \mu)$

BULK MODULUS (K)

CREEP CONSTANT (A)

STRESS EXPONENT (m)

THERMAL CONSTANT $\left(\frac{Q}{R \Theta}\right.$ if isothermal or $\frac{Q}{R}$ if not)

SHEAR EXPONENT $\left(\mu_{1}\right)$

BULK EXPONENT $\left(K_{1}\right)$

B0

B1

A1

INTACT DENSITY $\left(\rho_{\text {intact }}\right)$

INITIAL DENSITY $\left(\rho_{0}\right)$

11. M-D CREEP MODEL (number of cues =20)

TWO MU $(2 \mu)$

BULK MODULUS (K)

Al

Q1/R

N1

B1

A2

Q2/R

N2

B2

$\operatorname{SIGO}\left(\sigma_{0}\right)$

QLC (q)

$\mathrm{M}(\mathrm{m})$

$\mathrm{KO}$

$\mathrm{C}(c \Theta)$

$\operatorname{ALPHA}\left(\alpha_{w}\right)$

$\operatorname{BETA}\left(\beta_{w}\right)$

DELTLC $(\delta)$ 
RN3 (exponent of workhardening and recovery term used to compute $F$ )

AMULT (scalar multiplier of time step needed for stability, default 0.98 )

Examples for the ELASTIC PLASTIC material are given below to illustrate how the user might input the data in different forms. All three examples are identical as far as SANTOS is concerned.

Example 1:

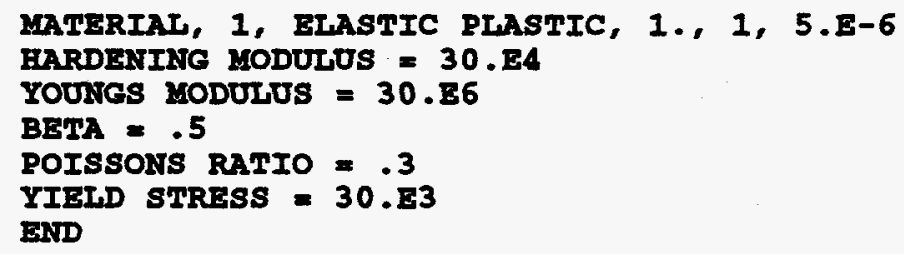

Example 2:

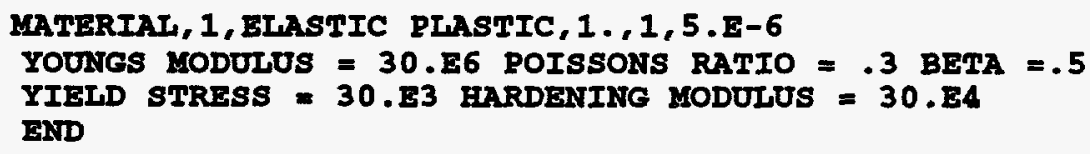

Example 3:

MATERIAL, 1, ELASTIC PLASTIC, 1, 1, 5.E-6

YOUNGS MODULUS $=30 . \mathrm{E} 6$ POISSONS RATIO $=.3 \mathrm{BETA}=.5$

YIELD STRESS $=30 . \mathrm{E} 3$ HARDENING YODULUS $=30 . \mathrm{E} 4$ END

42. MATERIAL POINT, $x, y$

$x, y$... coordinates of a material point which will be monitored and printed at the output intervals,

43. DELETE MATERIAL, material id, deletion time

material id ... material identification number

deletion time ... time at which all elements made up of this material should be deleted from the mesh.

Example Input File:

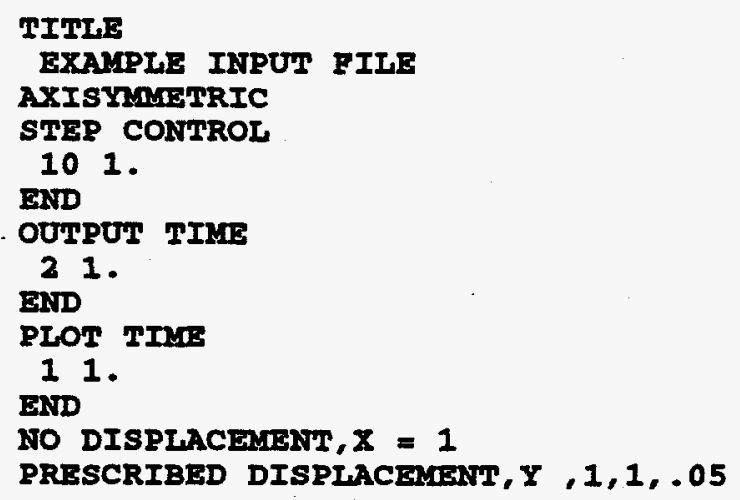


FUNCTION, 1

0,0

$1 ., 1$.

END

MATERIAL, 1, EIASTIC PLASTIC,1. \$ 21-6-9 stainless steel

YOUNGS MODULUS $=29.4+6$ POISSONS RATIO=.3

YIELD STRESS $=58.0 \mathrm{~B}+3$ RARDENIMG MODULUS $=29 \cdot 4 E+4$ BETA $=1$.

END

MATERIAL,2, EIASTIC, 1. \$ Tape Jolnt Filler (stee1)

YOUTES YODUUUS $=29.4 \mathrm{~B}+6$ POISSONS RATIO=.3

END

MATERIAI, 3, EIASTIC,1. \$ Equivalent mess eloments gimulating romainder YOUNGS MODULUS $=30$.E6 POISSONS RATIO=.3 END

RIGID SURFACE $=100,0,0,0,0, \ldots 1$

CONTACT SURFACE $=101,102$

CONTACT SURFACE $=103,104$

CONTACT SURPACE $=105,106$

CONTACT SURFACE $=107,108$

EXIT 
APPENDIX B:

User Subroutines

B-1 
Intentionally Left Blank

B-2 


\section{User Subroutines}

SANTOS allows the user to supply their own subroutines for defining an initial stress state (SUBROUTINE INITST) and an adaptive pressure routine (SUBROUTINE FPRES). The initial stress feature is particulary useful for geomechanics applications where an overburden stress is a function of depth. The adaptive pressure capability has been successfully used to define the pressure acting on the walls of a deforming cavity based on an assumption of ideal gas behavior.

The call to SUBROUTINE INITST has the following form:

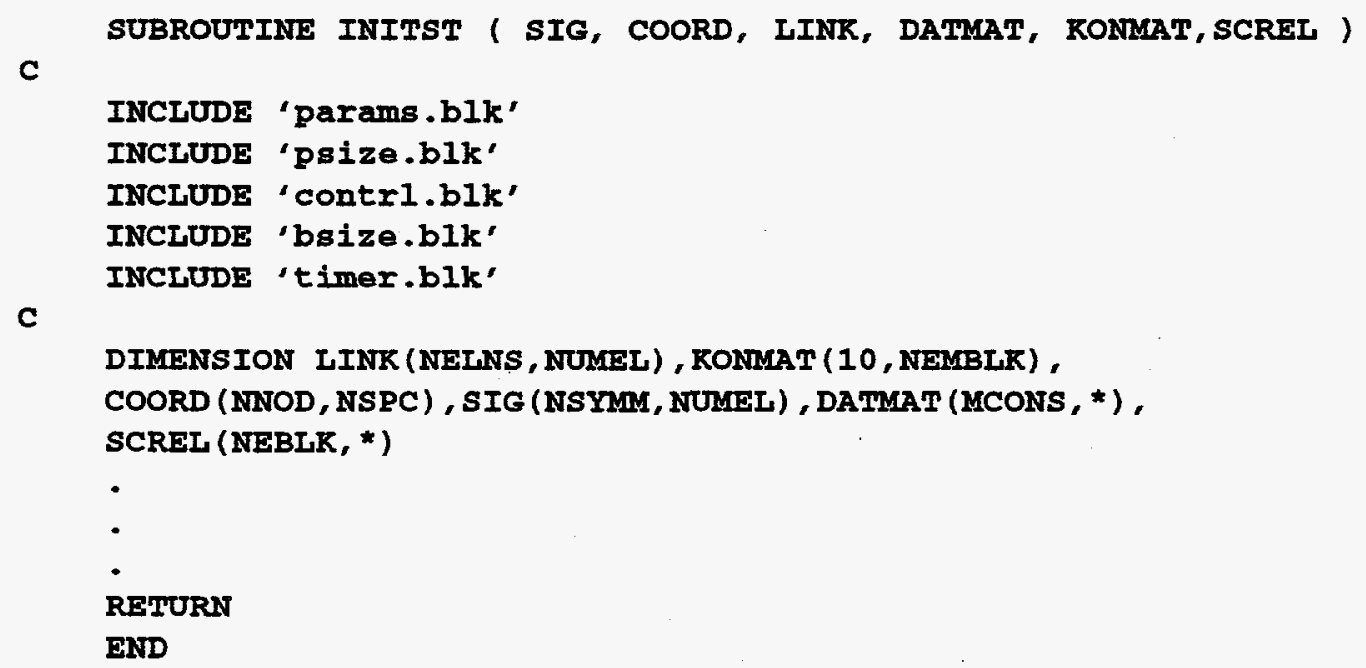

where the calling arguments are defined as:

SIG - element stress array which must be returned with the required stress values COORD - global nodal coordinate array

LINK - element connectivity array

DATMAT - material property array

KONMAT - material property integer array

SCREL - scratch element storage space

The arguments contained in the DIMENSION statement are located in the COMMON blocks which will be included during the program MAKE operation. If the MAKE utility is not used then the COMMON blocks will have to be explicitly included. This subroutine is called once prior to beginning the calculation by SUBROUTINE INIT. An example of a typical routine used for a geomechanics application is shown below. In this example, only material \#1 is being initialized with respect to depth. Other materials in the problem are having their initial stresses set to zero. 
SUBROUTINE INITST ( SIG, COORD, IINK, DATMAT, KONLAT, SCREL )

C

C

C

C DESCRIPTION:

C THIS ROUTINE PROVIDES AN INITIAL STRESS STATE TO SANTOS

C

C FORMAL PARAMETERS:

C SIG REAL ELEMENT STRESS ARRAY WEICH MUST BE RETURNED

C WITH THE REQUIRED STRESS VALUES

C COORD REAL GLOBAL NODAL COORDINATE ARRAY

C IINK INTEGER CONNECTIVITY ARRAY

C DATMAT REAL MATERIAL PROPERTIES ARRAY

C KONMAT INTEGER MATERIAL PROPERTIES INTEGER ARRAY

C

C CALLED BY: INIT

C

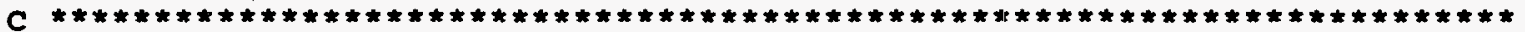

C

INCLUDE 'params.bIk'

INCLUDE 'psize.blk'

INCLUDE 'contrl.blk'

INCLUDE 'bsize.blk'

INCLUDE 'timer.bIk'

c

DIMENS ION LINK (NELNS, NUNEL), KONMAT (10, NEMBILK),

COORD (NNOD, NSPC) , SIG (NSYMM, NUMEL), DATMAT (MCONS , *),

SCREL (NEBLK, *)

C

DO $1000 I=1$, NEMBLK

MATID $=\operatorname{KOMMAT}(1, I)$

MRIND $=\operatorname{KONMAT}(2, I)$

$\operatorname{ISTRT}=\operatorname{KONMAT}(3, I)$

IEND $=\operatorname{KOMMAT}(4, I)$

IF ( MATID . EQ. 1 ) THEN

DO $500 \mathrm{~J}=$ ISTRT, IEND

$I I=\operatorname{IINK}(1, J)$

$J J=\operatorname{IINK}(2, J)$

$\mathrm{KK}=\operatorname{IINK}(3, J)$

IL $=\operatorname{IINK}(4, J)$

ZAVG $=0.25 *(\operatorname{COORD}(I I, 2)+\operatorname{COORD}(J J, 2)+\operatorname{COORD}(K K, 2)+$ COORD (LL, 2) )

STRESS $=-2.256 \mathrm{E} 4 *(655$. ZAVG $)$

$\operatorname{SIG}(1, J)=$ STRESS

$\operatorname{SIG}(2, J)=$ STRESS.

$\operatorname{SIG}(3, J)=$ STRESS

$\operatorname{SIG}(4, J)=0.0$

500 CONTINUE

ELSE

DO $600 \mathrm{~J}=$ ISTRT, IEND

$\operatorname{SIG}(1, J)=0.0$

$\operatorname{SIG}(2, J)=0.0$

$\operatorname{SIG}(3, J)=0.0$

$\operatorname{SIG}(4, J)=0.0$ 
END

The call to SUBROUTINE FPRES has the following form:

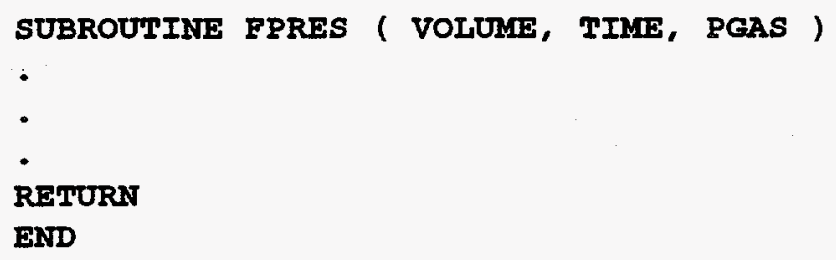

where the calling arguments are defined as:

VOLUME - computed volume of the cavity

TIME - current analysis time

PGAS - calculated gas pressure to be returned to calling program

This subroutine is called each iteration from SUBROUTINE EXLOAD. An example of a typical routine used for a geomechanics application is shown below. In this example, the volume coming into the subroutine corresponds to one-quarter of the total cavity volume due to the use of symmetry modeling conditions. The gas generation rate varies with time. The volume is multiplied by 4 to get the correct volume and the volume of solids is subtracted to get the free volume. The ideal gas law is then used to compute the internal cavity gas pressure.

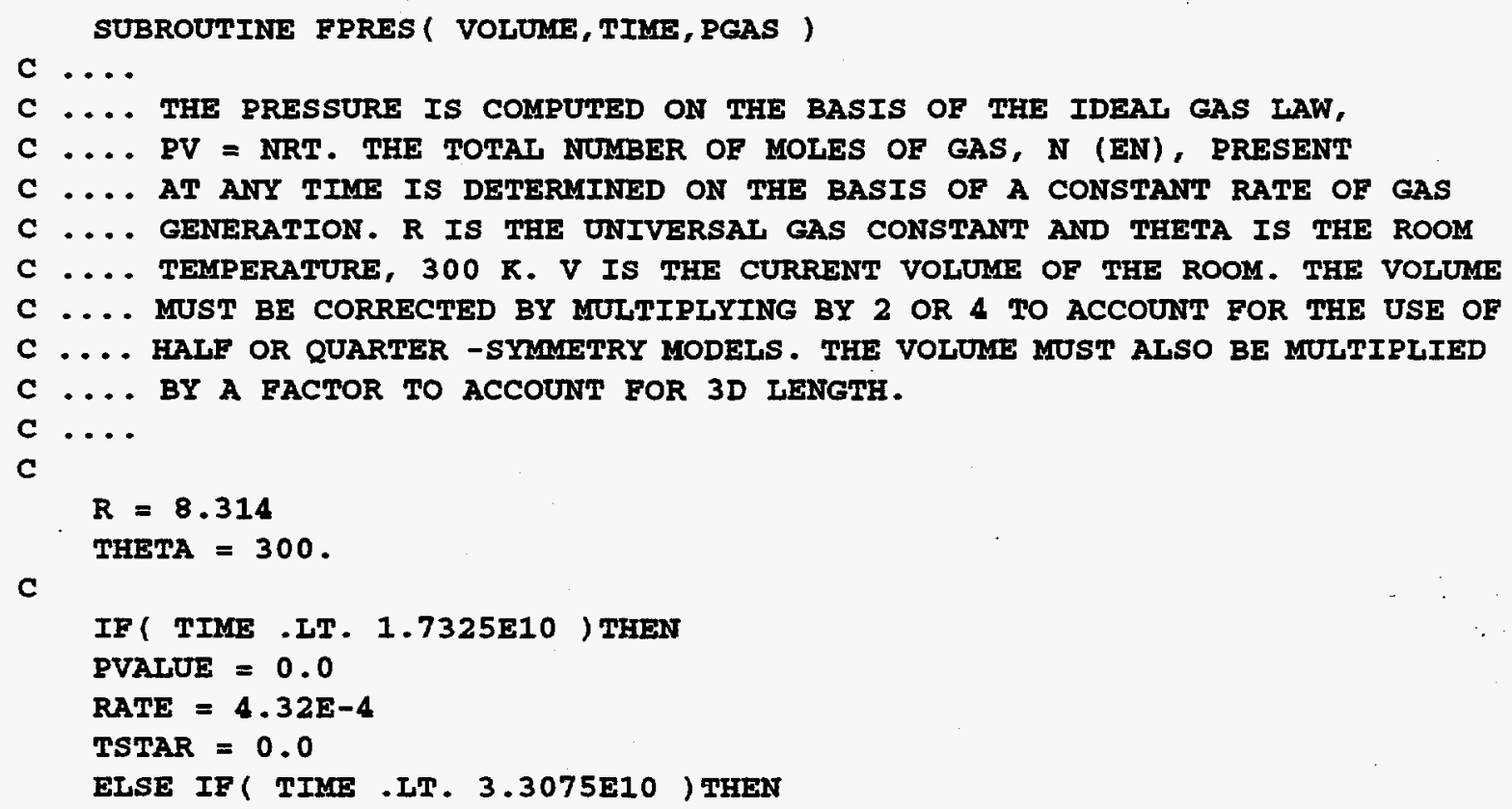




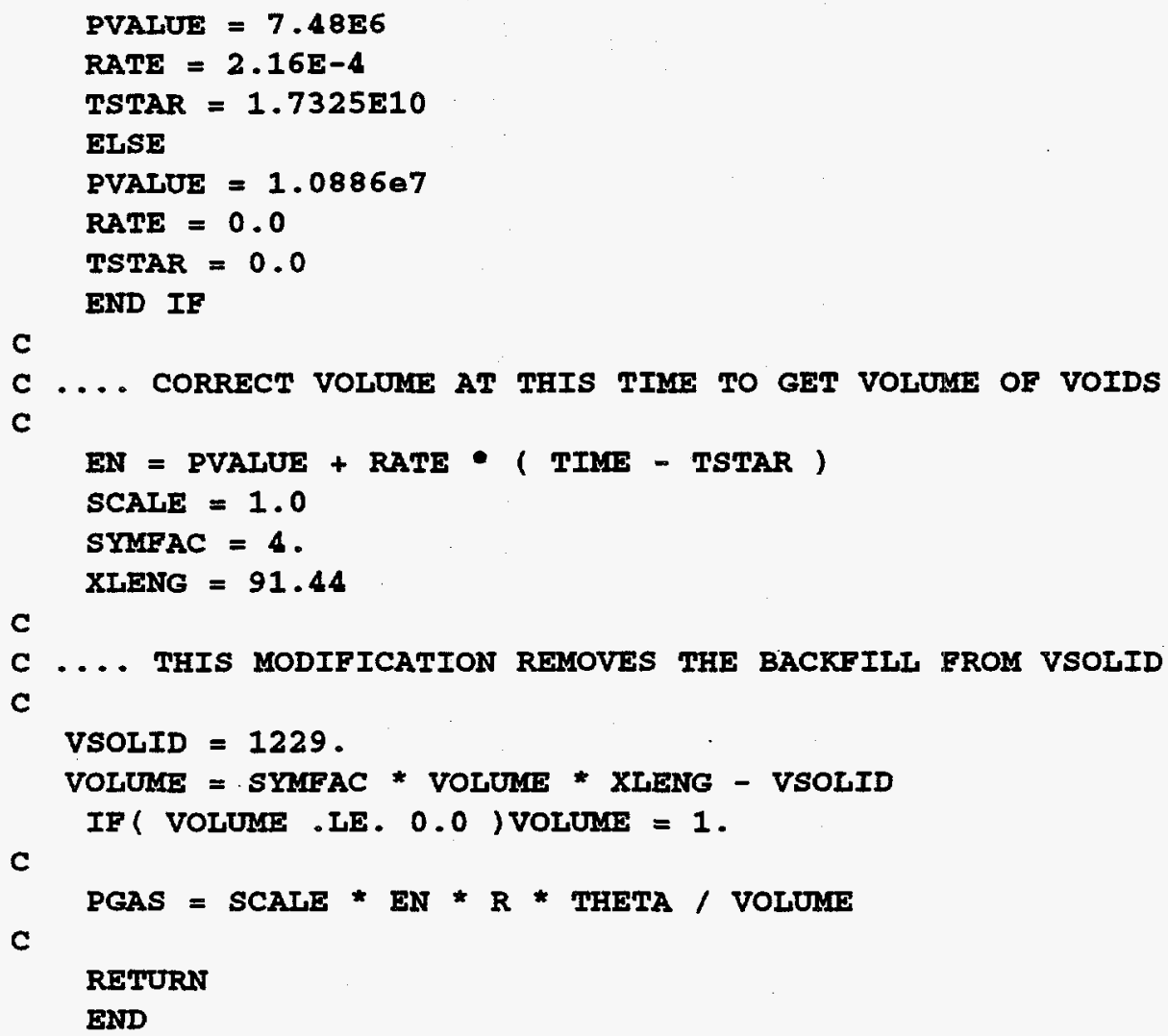

Be 
APPENDIX C:

Printed Output Description

C-1 
Intentionally Left Blank 


\section{Printed Output Description}

The SANTOS printed output begins with an echo of the input data stream from input unit 5 . This is followed by the PROBLEM DEFINITION section that lists the number of elements, nodal points, number of materials, analysis type, etc. that pertain to defining the problem to be solved. The information presented in this section also includes the solution algorithm parameters such as convergence tolerances, houglass stiffness and viscosity values, and the effective modulus status. The amount of output written in this section depends on the analysis type and the options requested. An example of this output for a sample analysis is shown below.

\section{P R O B I E M D E E I N I T I O N}

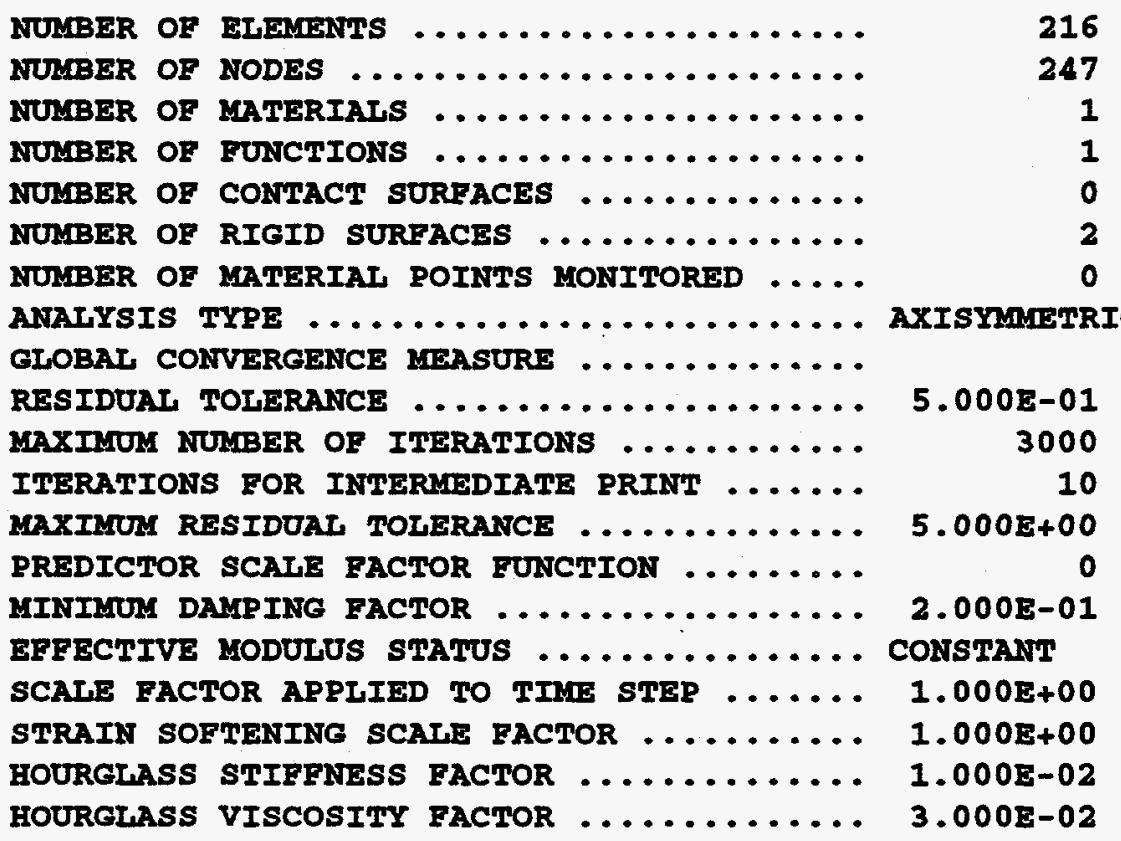

Following the PROBLEM DEFINITION section are the definitions of the load steps, printed output frequency and the plotted output frequency. A sample output for these sections is shown below. LOAD STEP DEFINTIONS shows the number of steps taken between each defined time interval. The PRINTED OUTPUT FREQUENCY data echo shows the number of load steps between printed output dumps during the defined time interval. PLOTTED OUTPUT FREQUENCY echos the number of load steps between plot dumps during the defined time interval. 


$\begin{array}{lrc}\text { TIME } & \text { NO. OF STEPS } & \text { TIME } \\ 0.000 \mathrm{E}+00 & 100 & 1.000 \mathrm{E}+00\end{array}$

P R I N T E D O U T P U T F R E Q U E N C Y

$\begin{array}{ccc}\text { TIME } & \text { STEPS BETWEEN PRINTS } & \text { TIME } \\ 0.000 \mathrm{E}+00 & 1 & 1.000 \mathrm{E}+00\end{array}$

P I O T T E D O U T P U T E R E Q U E N C Y

TIME

$0.000 \mathrm{E}+00$
STEPS BETWEEN PLOTS

1
TIME

$1.000 \mathrm{E}+00$

The next output grouping echos back the material type and material constants. Some additional constants which are computed by SANTOS within the constitutive model pre-processor are also printed.

M A TER I A L D E F I N I T I O N S

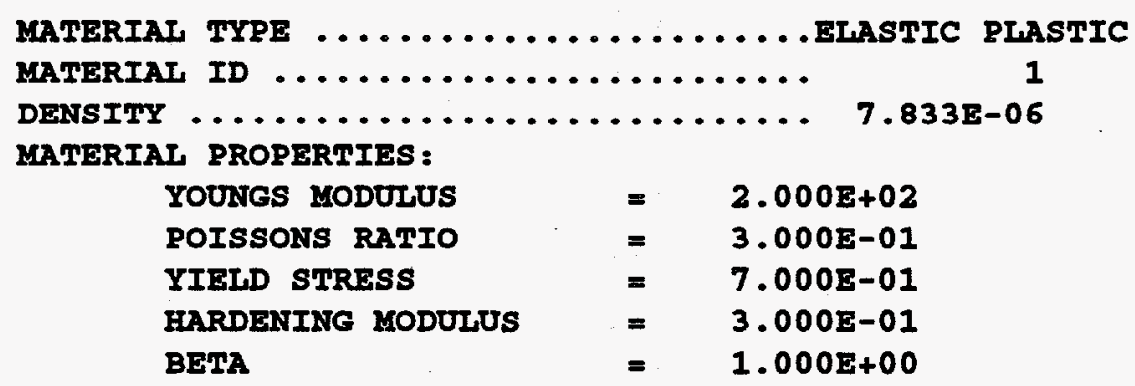

The next section of output echos back the processed input data regarding kinematic and traction boundary condition data. This data also includes function specifications and contact surface definitions. Distinction is made between NO DISPLACEMENT and PRESCRIBED DISPLACEMENT kinematic boundary conditions. 


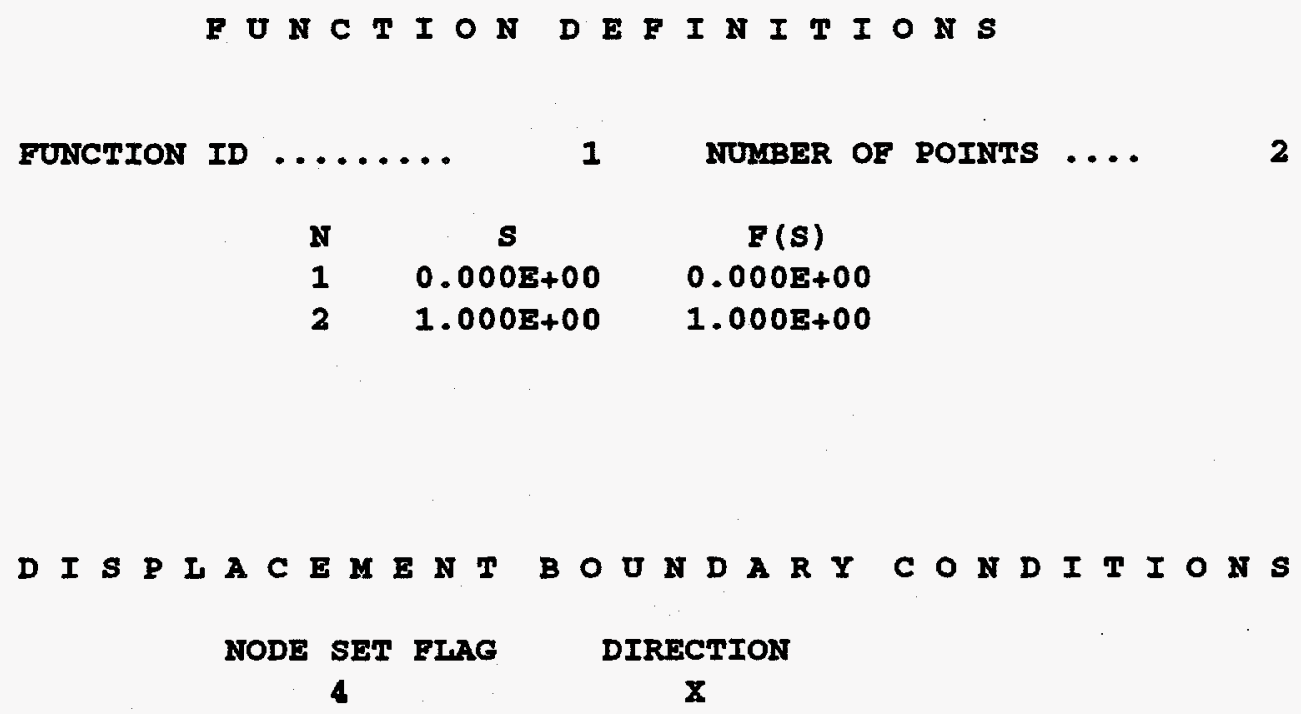

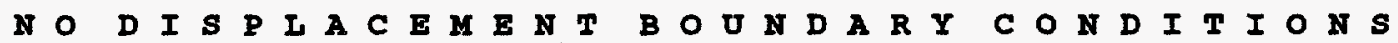
4

$\mathbf{x}$

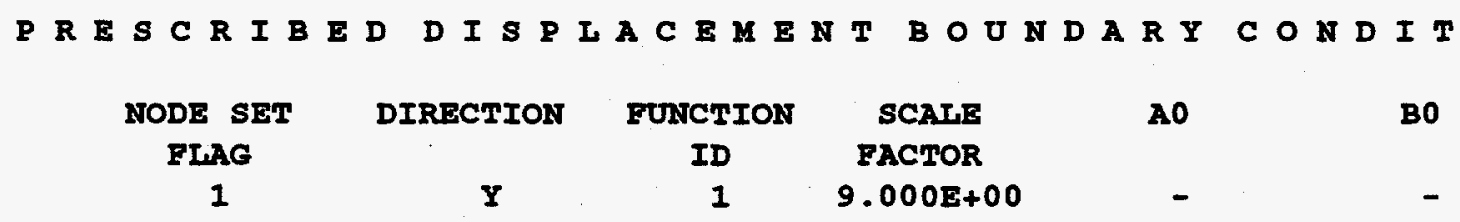

R I G I D S O R E A C E S

$\begin{array}{ccl}\text { SURFACE } & \text { SIDE SET } & \text { COEREICIENT } \\ \text { NUMBER } & \text { FIAG } & \text { OF FRICTION } \\ 1 & 300 & \text { FIXED } \\ 2 & 200 & \text { FIXED }\end{array}$

XO YO $\mathbf{Y X} \quad \mathbf{N Y}$

$0.000 \mathrm{E}+00 \quad 1.500 \mathrm{E}+01 \quad 0.000 \mathrm{E}+00-1.000 \mathrm{E}+00$

$0.000 \mathrm{E}+001.500 \mathrm{E}+01 \quad 0.000 \mathrm{E}+00-1.000 \mathrm{E}+00$

The next grouping defines the quantities written to the plotting data base. The plotted output is grouped by whether the variable being written is a nodal, element, or global quantity. The global quantities, FX and FY, written to the data base refer to the sum of the applied loads in the $\mathrm{x}$ and $\mathrm{y}$-directions, respectively. The quantities, $\mathrm{RX}$ and $\mathrm{RY}$, refer to global reaction forces in the $\mathrm{x}$ and $y$-directions summed at nodes specified to have NO DISPLACEMENT boundary conditions applied. For axisymmetric analyses, the forces FX, FY, RX, and RY are output per radian. The nodal variables, RESIDX RESIDY RESID, refer to imbalance or residual forces acting at the nodes. The variables RESIDX and RESIDY refer to the $\mathrm{x}$ and $\mathrm{y}$-component directions of the imbalance forces, respectively. The variable RESID is the scalar magnitude of the components. Material model state variables appear as element variables in the plotting data base. 


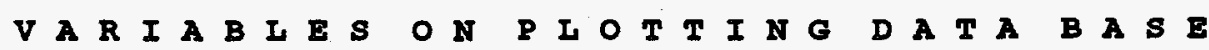

\begin{tabular}{lll} 
NODAI & ELEMFNT & GLOBAL \\
\hline DISPLX & RRESSURE & FX \\
DISPLY & VONMISES & FY \\
RESIDX & EQPS & RX \\
RESIDY & & RY \\
RESID & & ITER
\end{tabular}

If the INTERMEDIATE PRINT option is in effect then the following output is obtained every $n$ iterations. For this example, $n$ is specified to be every 10 iterations. The values under the STEP column refer to the number of iterations taken relative to this load step. The column labeled TIME shows the problem time for which an equilibrium solution is being sought. The column labeled TIME STEP shows the stable time step internally computed within SANTOS which is being used to integrate the equations of motion. This number may change from one iteration to the next as the element is deformed. The column labeled DAMPING FACTOR provides the current adaptive dynamic relaxation damping parameter. The next two columns provide information regarding convergence of the load step. The APPLIED LOAD NORM refers to the L2 norm of the externally applied loads while the RESIDUAL LOAD NORM is the L2 norm of the imbalance forces at each node. The PERCENT IMBALANCE column is the result of dividing the RESIDUAL LOAD NORM by the APPLIED LOAD NORM which is the measure used to determine convergence of the iterative scheme. The column defined as TOTAL STEPS gives a running total of the number of iterations for the problem.

\begin{tabular}{|c|c|c|c|c|c|c|c|}
\hline TEP & TIME & $\begin{array}{l}\text { TIME } \\
\text { STEP }\end{array}$ & $\begin{array}{l}\text { DAMPING } \\
\text { FACTOR }\end{array}$ & $\begin{array}{l}\text { APPLIED } \\
\text { LOAD NORM }\end{array}$ & $\begin{array}{l}\text { RESIDUAL } \\
\text { LOAD NORM }\end{array}$ & $\begin{array}{c}\text { PERCENT } \\
\text { IMBALANCE }\end{array}$ & $\begin{array}{l}\text { TOTAL } \\
\text { STEPS }\end{array}$ \\
\hline 10 & $1.000 \mathrm{E}-02$ & $9.984 \mathrm{E}-03$ & $7.105 E-01$ & $2.927 E+01$ & $1.047 \mathrm{E}+02$ & 357.71 & 10 \\
\hline 20 & $1.000 \mathrm{E}-02$ & $9.991 z-03$ & $7.024 E-01$ & $2.395 E+01$ & $2.456 \mathrm{E}+01$ & 102.55 & 20 \\
\hline 30 & $1.000 \mathrm{E}-02$ & $9.994 \mathrm{E}-03$ & $5.261 \mathrm{E}-01$ & $1.748 E+01$ & $7.866 \mathrm{E}+00$ & 44.99 & 30 \\
\hline 40 & $1.000 \mathrm{E}-02$ & $9.996 \mathrm{E}-03$ & $9.896 \mathrm{E}-01$ & $1.600 \mathrm{E}+01$ & $4.727 E+00$ & 29.55 & 40 \\
\hline 50 & $1.000 \mathrm{E}-02$ & $9.997 \mathrm{E}-03$ & $9.574 \mathrm{E}-01$ & $1.311 \mathrm{E}+01$ & $2.591 E+00$ & 19.77 & 50 \\
\hline 60 & $1.000 \mathrm{E}-02$ & $9.998 \mathrm{E}-03$ & $9.069 \mathrm{E}-01$ & $1.259 \mathrm{E}+01$ & $2.011 E+00$ & 15.97 & 60 \\
\hline 70 & $1.000 \mathrm{E}-02$ & $9.998 \mathrm{E}-03$ & $9.428 \mathrm{E}-01$ & $1.236 \mathrm{E}+01$ & $1.604 \mathrm{E}+00$ & 12.97 & 70 \\
\hline 80 & $1.000 \mathrm{E}-02$ & $9.999 \mathrm{E}-03$ & $9.346 \mathrm{E}-01$ & $1.200 E+01$ & $1.096 \mathrm{E}+00$ & 9.13 & 80 \\
\hline 90 & $1.000 \mathrm{E}-02$ & $9.999 \mathrm{E}-03$ & $8.438 \mathrm{E}-01$ & $1.166 \mathrm{E}+01$ & $7.562 \mathrm{E}-01$ & 6.49 & 90 \\
\hline
\end{tabular}

The final output section to be described is the printed output that results when the iterative solution reaches equilibrium as measured by the PERCENT IMBALANCE. The printed output provides descriptive information about the problem such as when the problem was run, version of the software, title of the problem and a summary of information about the convergence of the load step. 
1 SANTOS, VERSION SANTOS 2.0 , RUN ON 01/20/95, AT 16:15:25

UPSETTING OF A CYLINDRICAL BILLET

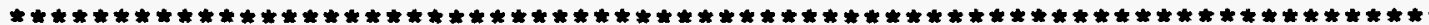

SUMMARY OF DATA AT STEP NOUBER

1, TIME $=1.000 \mathrm{E}-02$

NOMBER OF ITERATIONS $=$ 212, TOTAL NOMBER OF ITERATIONS $=$

FINAL CONVERGENCE TOLERANTE $=4.901 \mathrm{E}-01$

SUM OF EXTERNAL FORCES IN X-DIRECTION $=0.000 E+00$

SUM OF EXTERNAL FORCES IN Y-DIRECTION $=0.000 E+00$

SUM OF REACTION FORCES IN X-DIRECTION $=0.000 \mathrm{E}+00$

SUM OF REACTION FORCES IN Y-DIRECTION $=-3.561 \mathrm{E}+01$

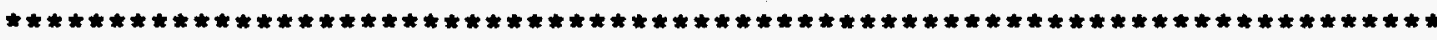

**** PLOT TAPE WRITTEN AT TIME $=1.000 \mathrm{~T}-02$ STEP NUTABR

$1 * * * *$ 
Intentionally Left Blank 


\section{APPENDIX D:}

Adding a New Constitutive Model to SANTOS 
Intentionally Left Blank

D-2 


\section{Adding A New Constitutive Model to SANTOS}

A material interface subroutine has been incorporated which allows the constitutive model developer to add a new material model with very little effort. The interface has been designed so that the developer does not need to understand the internal workings of SANTOS especially with respect to allocation and management of computer memory. If the developer follows the instructions in subroutine MATINT, then SANTOS will handle all memory allocation, material data reading, and material data printing. There are three steps that should be followed when adding a new model.

\section{$\underline{\text { Step } 1}$}

Subroutine MATINT contains instructions using FORTRAN comment cards which outline the steps that should be followed to add a new material model. Most of the required changes involve adding or changing numbers in DATA and PARAMETER statements. Since we have no prior knowledge of what the material constants represent for a particular material, the code requires that a few lines of FORTRAN be added to compute the initial dilatational modulus $(\lambda+2 \mu)$ and the initial shear modulus $(2 \mu)$ for the material. The dilatational modulus and the shear modulus must be stored in the variables DATMOD and SHRMOD, respectively. At this same location in the code it is possible to calculate any combination of the input parameters that may be required in the constitutive subroutine (e.g. bulk modulus from Young's modulus and Poisson's ratio).

There is a restriction to twenty characters in the material name, material cues, and internal state variable names which are defined in subroutine MATINT. The names may have blanks which means that multiple word cues are allowed. The names must be defined such that each word in the name is unique to the first three characters. This means that material cues $\mathrm{C} 1, \mathrm{C} 2, \mathrm{C} 3$, etc., are legal; but CON1, CON2, CON3, etc., are not.

\section{Step 2}

This step is optional and is only required if the new material model contains internal state variables which must be initialized to some value other than zero (all internal state variables are initialized to zero by default). If state variables must be initialized, an ELSE IF statement must be added to subroutine SVINIT for this material. This statement should read:

ELSE IF( MKIND .EQ. (new material number) ) THEN

initialize internal state variables here

This new material number corresponds to the position where the material resides in the list of 
material names defined in subroutine MATINT. Generally, when adding a new material, the new material is the last one defined and its number will be the same as the number of materials defined in STEP 1. The application of this step should be obvious from inspecting the coding of the other material models. Please use comments to record changes to the code.

\section{$\underline{\text { Step } 3}$}

In subroutine UPDSTR, the call to the new material model must be added. The material subroutine may have any appropriate name, but current convention has been to name the material subroutines MAT1, MAT2, MAT3, etc., where the number corresponds to the material number defined in Step 2. The call is included by adding an ELSE IF block to subroutine UPDSTR which should read:

ELSE IF( MKIND .EQ. (new material number) ) THEN

CALL new subroutine( ..... argument list ....)

The application of this step should be obvious from inspecting the coding of the other material models. Please use comments to record changes to the code. 
APPENDIX E:

Verification and Sample Problems 
Intentionally Left Blank 


\section{Verification and Sample Problems}

Sample problems are included to demonstrate code verification and to acquaint the user with the SANTOS program. The problems were selected to exercise and demonstrate many of the major features and options in the code.

\section{Large Deflection Analysis of a Cantilever Beam}

The large deformation of an elastic cantilever beam is included for comparison with the analytical solution as formulated by Holden (1972). The beam problem is challenging for the uniform strain quadrilateral elements and for the dynamic relaxation (DR) algorithm. The beam has a length-tothickness ratio of 30. The beam material is assumed to be elastic with a Young's modulus of 1. $\times 10^{7} \mathrm{psi}$ and a Poisson's ratio equal to zero. Both gravity and normal pressure loading conditions are considered.

The first loading condition considered is the beam loaded with gravity, which keeps the direction of loading constant throughout the analysis. Following the notation and development of Holden, the equation for the slope of the beam is

$$
\frac{d^{2} \theta}{d \bar{s}^{2}}=-k \bar{s} \cos \theta,
$$

where $\theta$ is the angle between the beam neutral axis and the $\mathrm{x}$-axis; $\bar{s}=s / L$ is the normalized arc length along the beam neutral axis; $k=w \frac{L^{3}}{E I}$ is a nondimensional loading parameter; $L$ is the length of the beam; $w$ is the loading intensity (load per unit length); $E$ is Young's modulus; and $I$ is the beam's moment of inertia. This equation describes the finite deflection of uniform beams using the Euler-Bernoulli theory of bending subject to vertical (gravity) loading. Boundary conditions for the cantilevered beam are a specified zero rotation at the fixed end.

The normalized horizontal and vertical deflections of the free end of the beam are then given by

$$
h / L=\int_{0}^{1} \cos \theta d \bar{s}
$$

and

$$
\delta / L=\int_{0}^{1} \sin \theta d \bar{s},
$$

respectively. Equation E-1 is solved using a Runge-Kutta procedure, the integrations for deflections are computed using adaptive quadrature, and the results are checked by comparison to 
Holden's published solution. Figure E-1 shows a schematic of the beam geometry and boundary conditions. The beam has thirty elements along its length and four through the beam thickness. The nonlinear beam response is calculated with SANTOS (triangles and squares) and compared to Holden's published solution (solid line) in Figure E-2. The comparison for this case is excellent. The deformed shape of the beam corresponding to $k=0 ., 6.5$, and 20. is shown in Figure E-3.

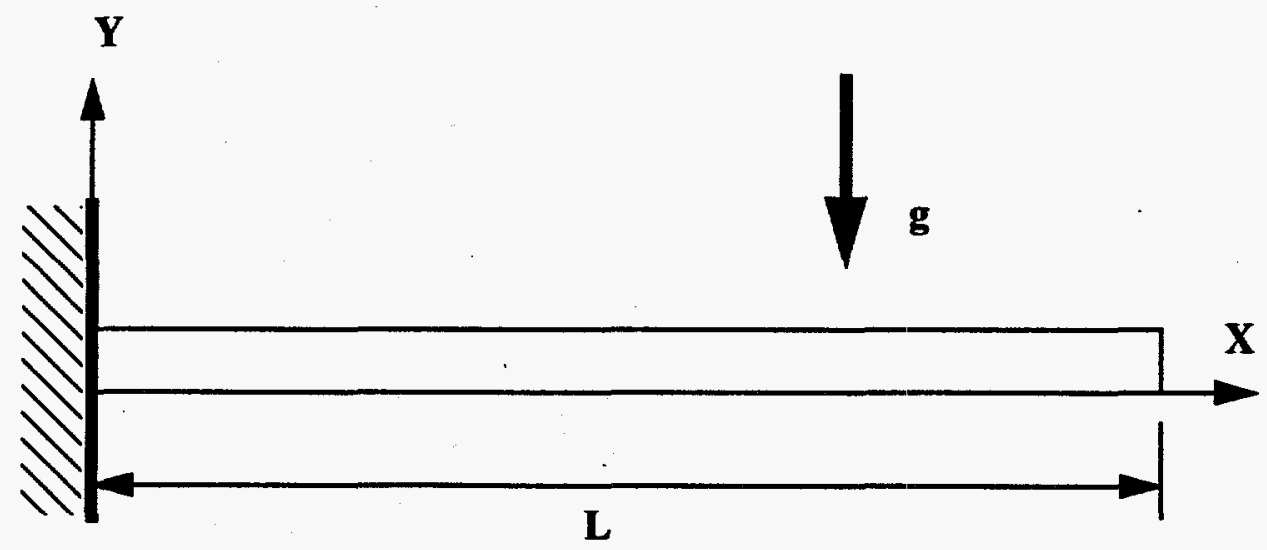

Figure E-1. Schematic of Cantilever Beam With Gravity Loading Showing the Geometry and Boundary Conditions

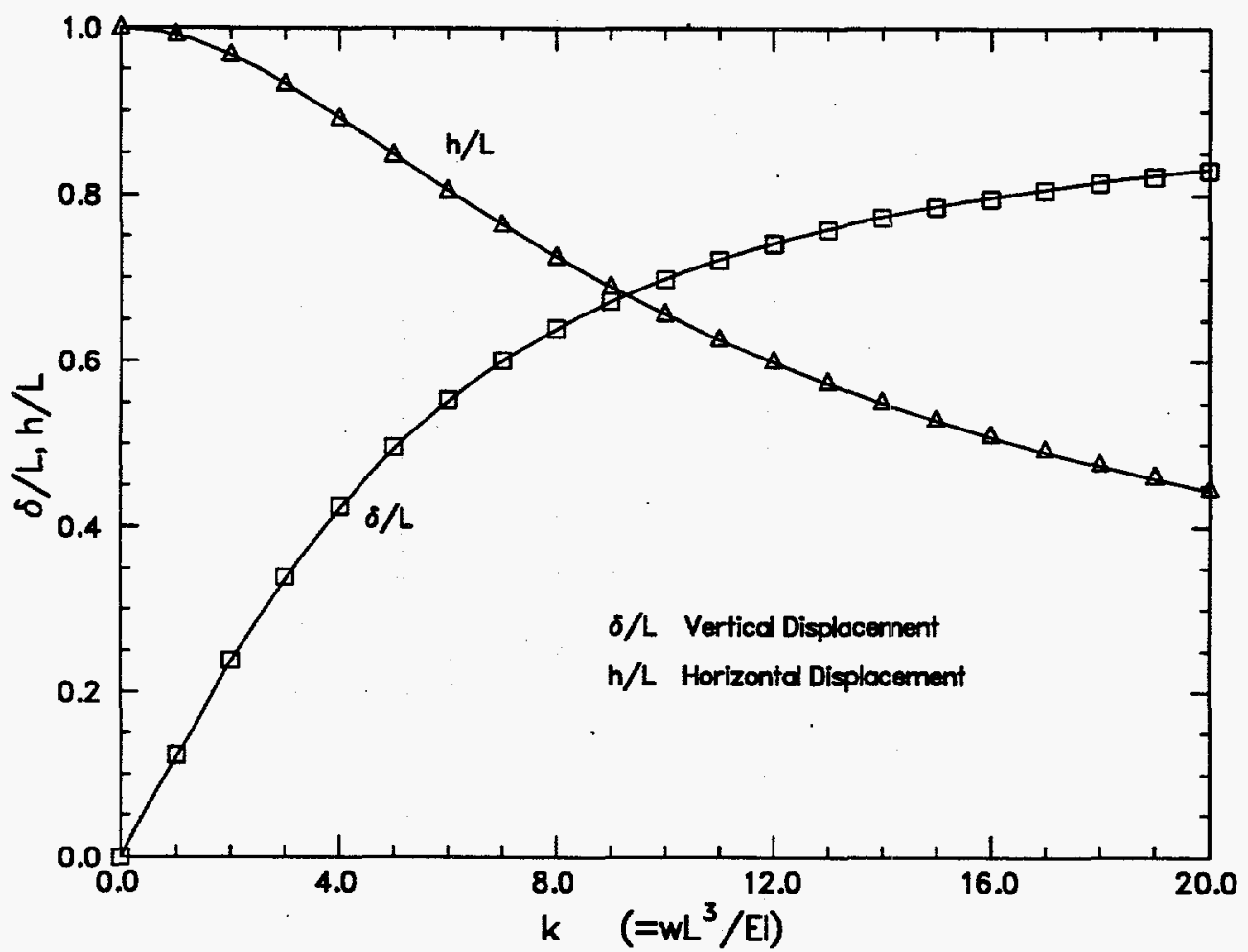

Figure E-2. Comparison of Analytic (solid line) and SANTOS (triangles and squares) Tip Displacements for the Beam With Gravity Loading. 
To obtain a solution to the gravity loaded beam problem using DR, we must make use of the NO DAMPING option. This option turns off the damping for a specified number of iterations which allows the beam to take on a more correct deformed shape before damping begins. In addition, this option is invoked only for the first 50 load steps which corresponds to the tip of the beam reaching a deflection magnitude equal to the thickness of the beam. Some large imbalance forces are experienced with the early load steps but these quickly disappear as the beam deforms and the deformation mode changes from small-deformation bending behavior to large-deformation bending behavior. A total of 310 load steps were taken for the gravity loaded case with each load step averaging 733 iterations. The SANTOS input file for the gravity loaded beam problem is shown in Figure E-4.

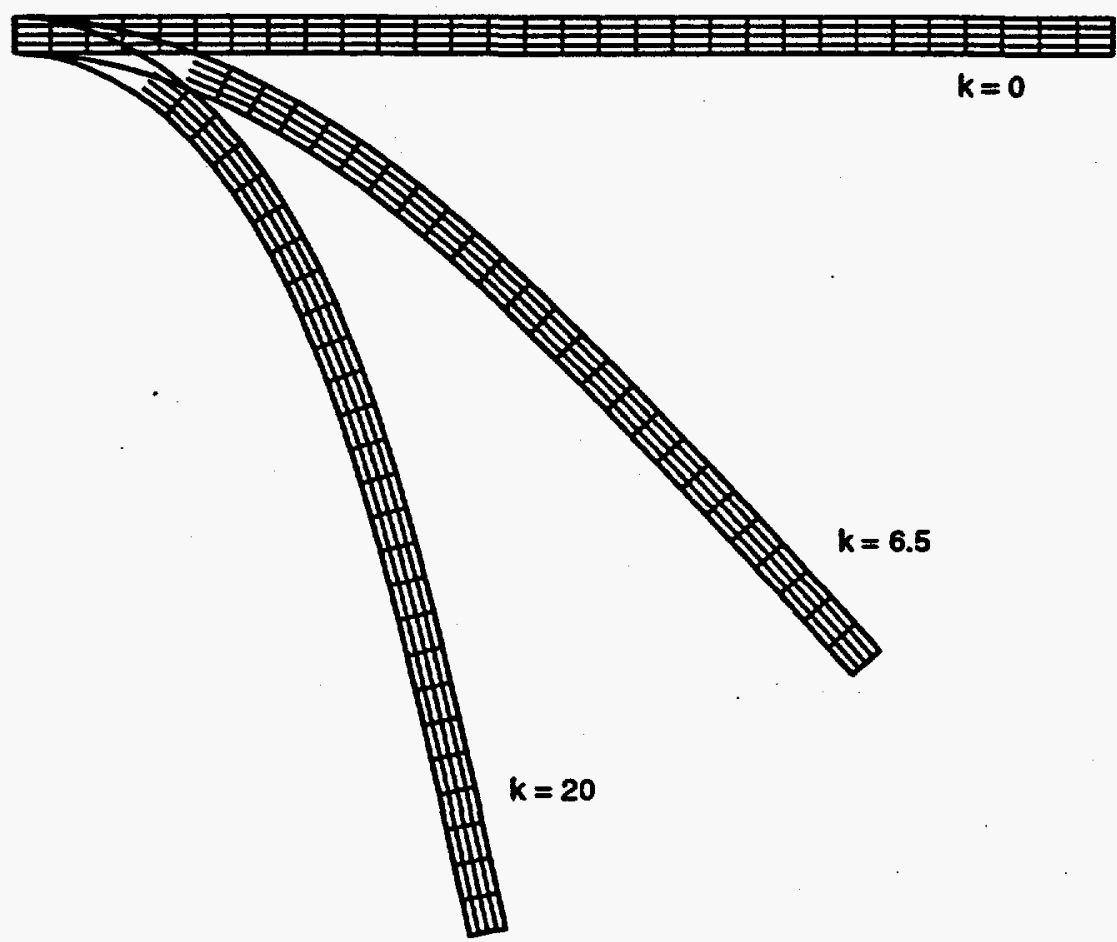

Figure E-3. Deformed Shape of the Beam Under Gravity Loading. Deformed Shapes Correspond to $\mathrm{k}=0.0,6.5$, and 20 .

The second loading condition is pressure applied along the top of the beam so that the loads remains normal to the surface throughout deformation. The beam theory equation for this case is

$$
\frac{d^{2} \theta}{d \bar{s}^{2}}=-k \bar{s},
$$

with the same boundary conditions as before. For large load magnitudes, this configuration causes more severe bending of the beam as shown in Figure E-5. The analytic solution (solid line) is compared to the SANTOS solution (triangles and squares) in Figure E-6. For this load case the finite element model is stiffer than the Euler-Bernoulli beam theory predicts at the higher loads. This difference is probably due to the fact that when the beam starts bending back on itself, the 


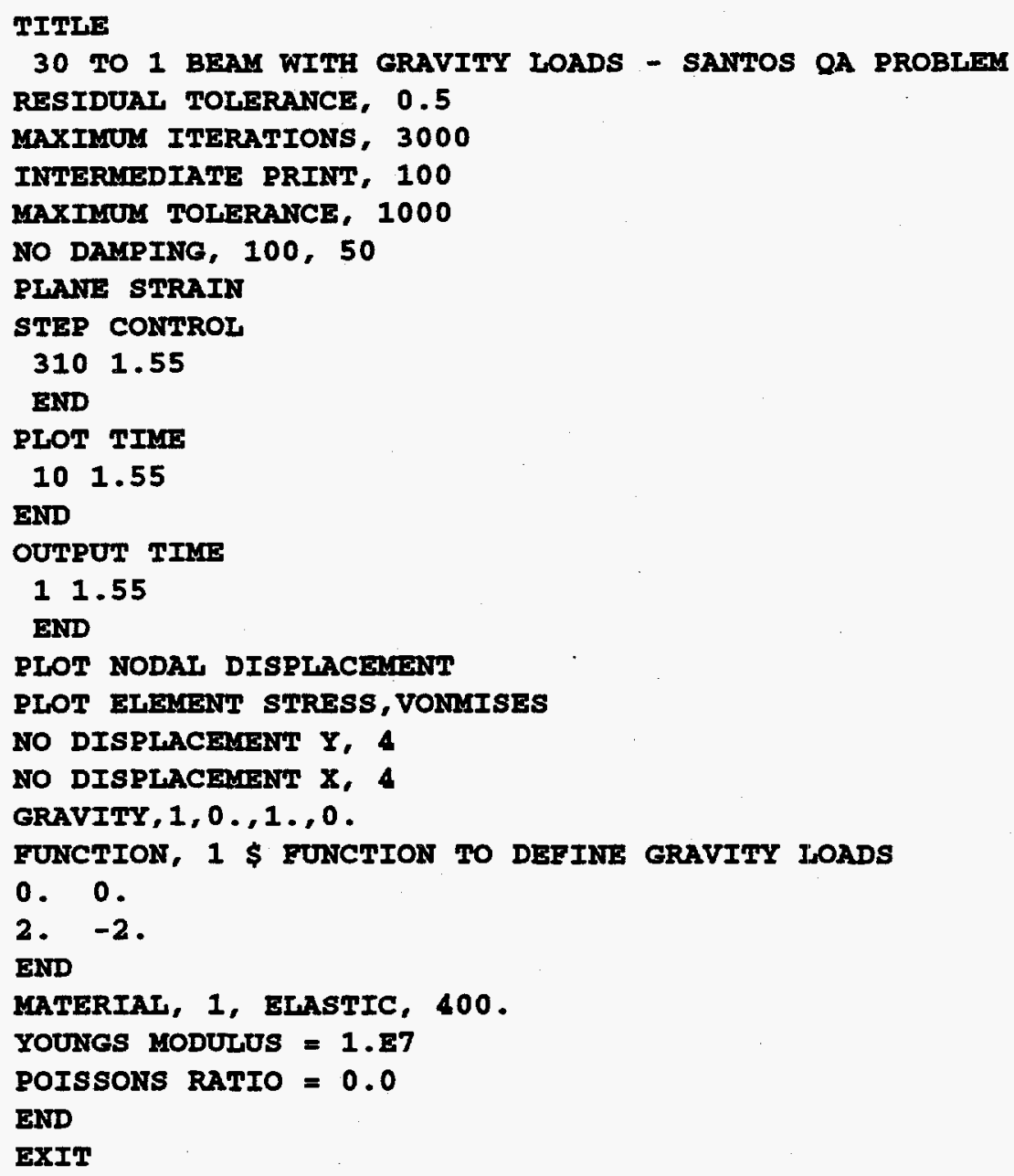

Figure E-4. SANTOS Input File for the Gravity Loaded Beam Verification Problem.

radius of curvature is no longer large compared to the thickness of the beam. The SANTOS solution employed 1550 load steps with an average of 270 iterations per load step. The input file for this load case is shown in Figure E-7.

\section{Elastic-Plastic Thick-Walled Hollow Sphere}

The problem of a thick-walled hollow sphere loaded into the plastic range by an internal pressure serves as a good check of the elastic-plastic material model. The two cases analyzed are for an elastic-perfectly plastic sphere and an elastic-plastic sphere with linear strain hardening. The sphere analyzed has an internal radius of one and an outer radius of two. The internal pressure is increased from the start of initial yield at the inner surface and is increased until the sphere becomes fully plastic. The problem is analyzed using the axisymmetric option in SANTOS. In addition, symmetry boundary conditions are assumed so that only a quarter of the sphere is modeled as shown in Figure E-8. The mesh discretization uses 30 elements spaced uniformly in the radial direction and 20 elements spaced uniformly around the circumference for a total of 600 elements. The sphere material has a Young's modulus of 207. GPa and a Poisson's ratio of 0.3 . The yield 


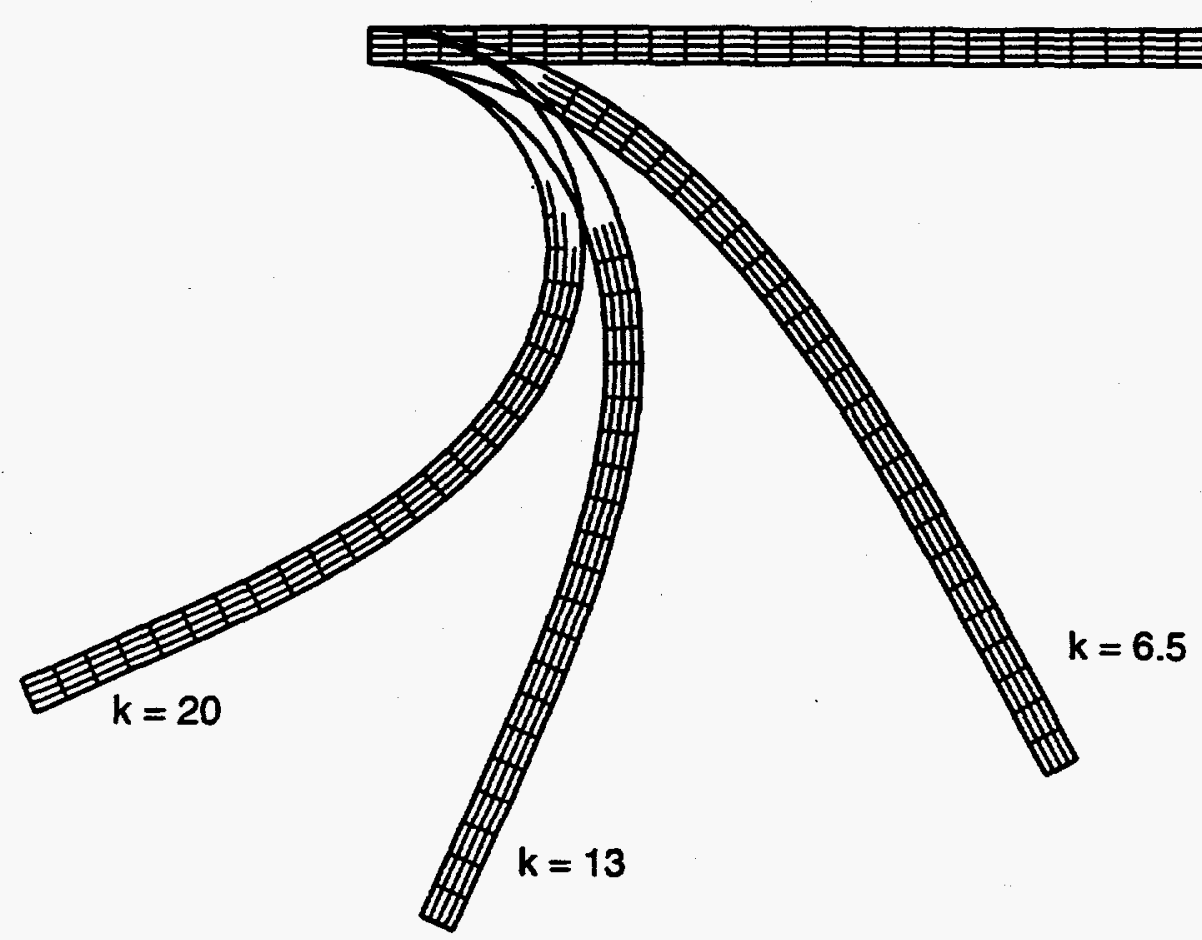

Figure E-5. Deformed Shape of the Beam With Applied Pressure Loading.

Deformed Shapes Correspond to $k=0.0,6.5,13$., and 20 .

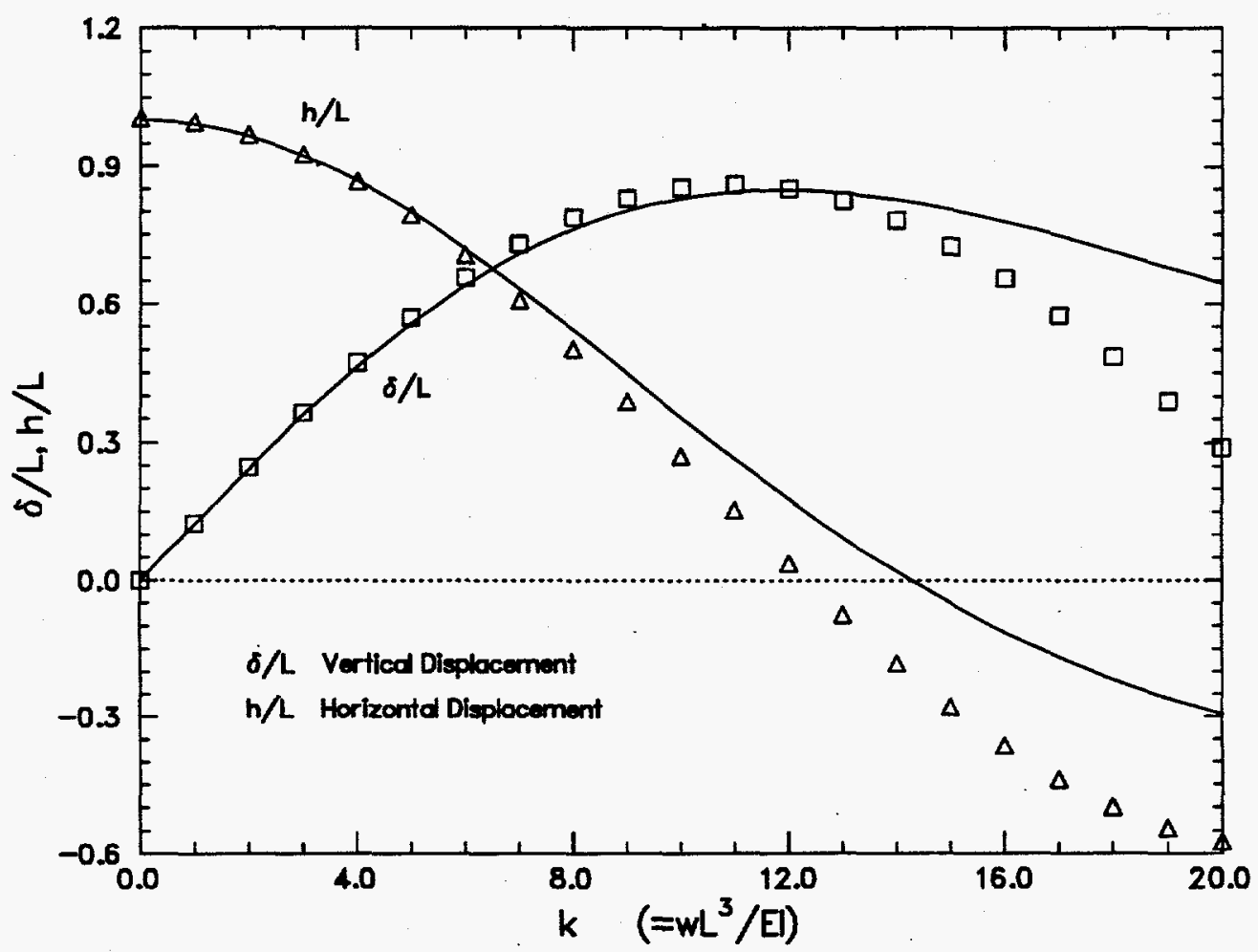

Figure E-6. Comparison of Analytic (solid line) and SANTOS (squares and triangles) Tip Displacements for the Beam With Applied Pressure Loading. 


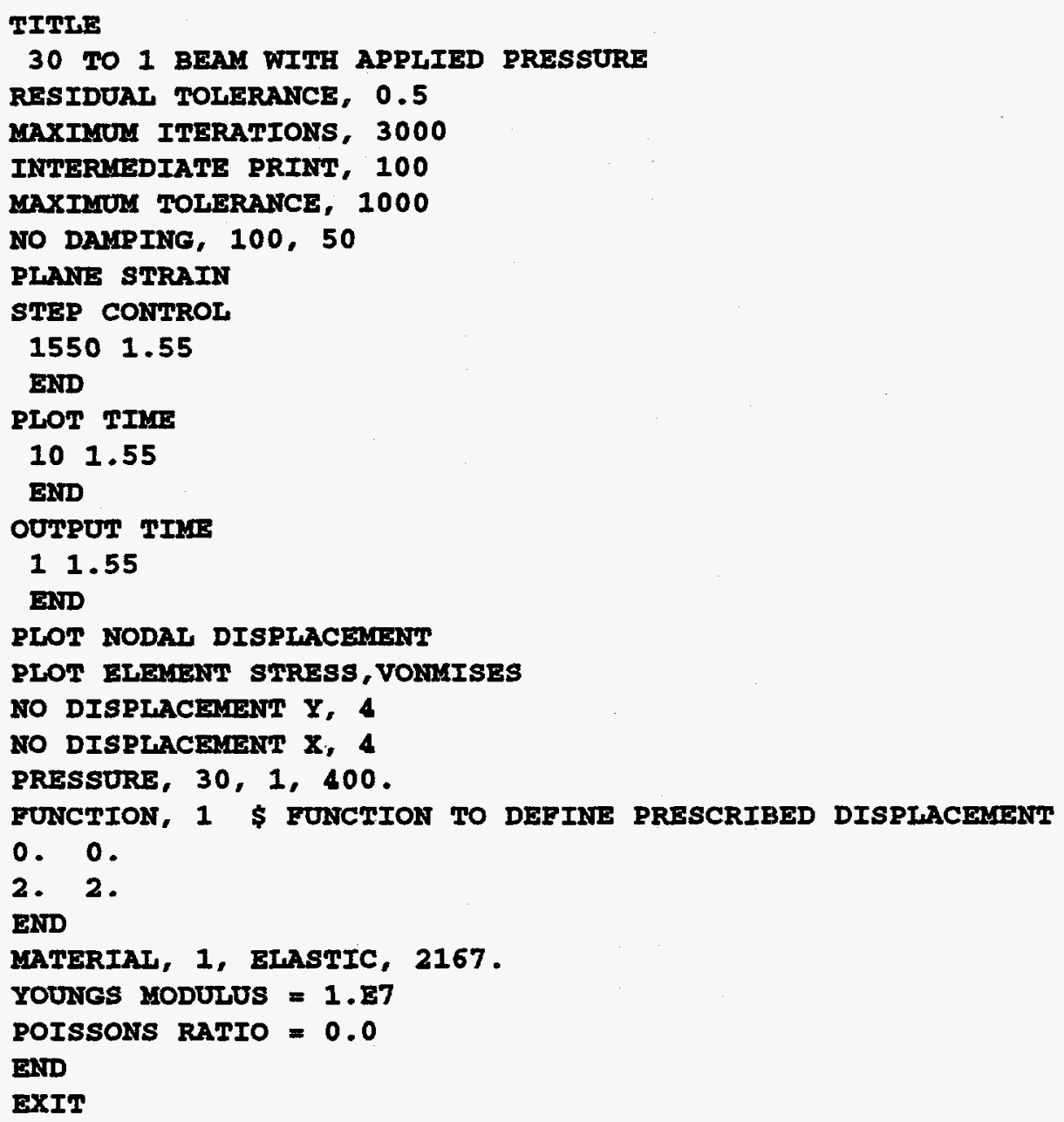

Figure E-7. SANTOS Input File for Pressure Loaded Beam Verification Problem.

stress is set to 10000 . and the hardening modulus is $20.7 \mathrm{GPa}$ for the linear strain hardening problem. The hardening modulus is set to zero for the elastic-perfectly plastic analysis.

The analytical solutions for these problems were derived by Mendelson (1968). For an internally pressurized sphere, the elastic/plastic interface expands radially outward from the inner surface of the sphere according to the following equations taken from Mendelson. The first relation is for the elastic perfectly-plastic material and defines the radius, $c$, of the elastic-plastic interface

$$
P=2 \ln \rho_{c}+\frac{2}{3}\left(1-\frac{1}{\beta_{c}^{3}}\right)
$$

and the second equation defines the elastic-plastic interface for the linear strain hardening material. 


$$
P=\frac{\frac{4 m}{3}(1-v)\left(1-\frac{1}{\beta^{3}}\right) \rho_{c}{ }^{3}+2(1-m) \ln \rho_{c}+\frac{2}{3}(1-m)\left(1-\frac{1}{\beta_{c}^{3}}\right)}{1-m+2 m(1-v)}
$$

The non-dimensional variables used in Equations E-5 and E-6 are: $P=p / \sigma_{y}$ is the ratio of applied internal pressure to material yield stress, $\sigma_{y} ; \rho_{c}=c / a$ is the ratio of the elastic-plastic interface radius to the sphere's internal radius, $a ; \beta_{c}=b / c$ is the ratio of the sphere's outer radius, $\mathrm{b}$, to the elastic-plastic interface radius; $\beta=b / a$ is the ratio of the sphere's outer to inner radii; $\mathrm{m}$ is the ratio of the hardening modulus to the Young's modulus; and $v$ is Poisson's ratio.

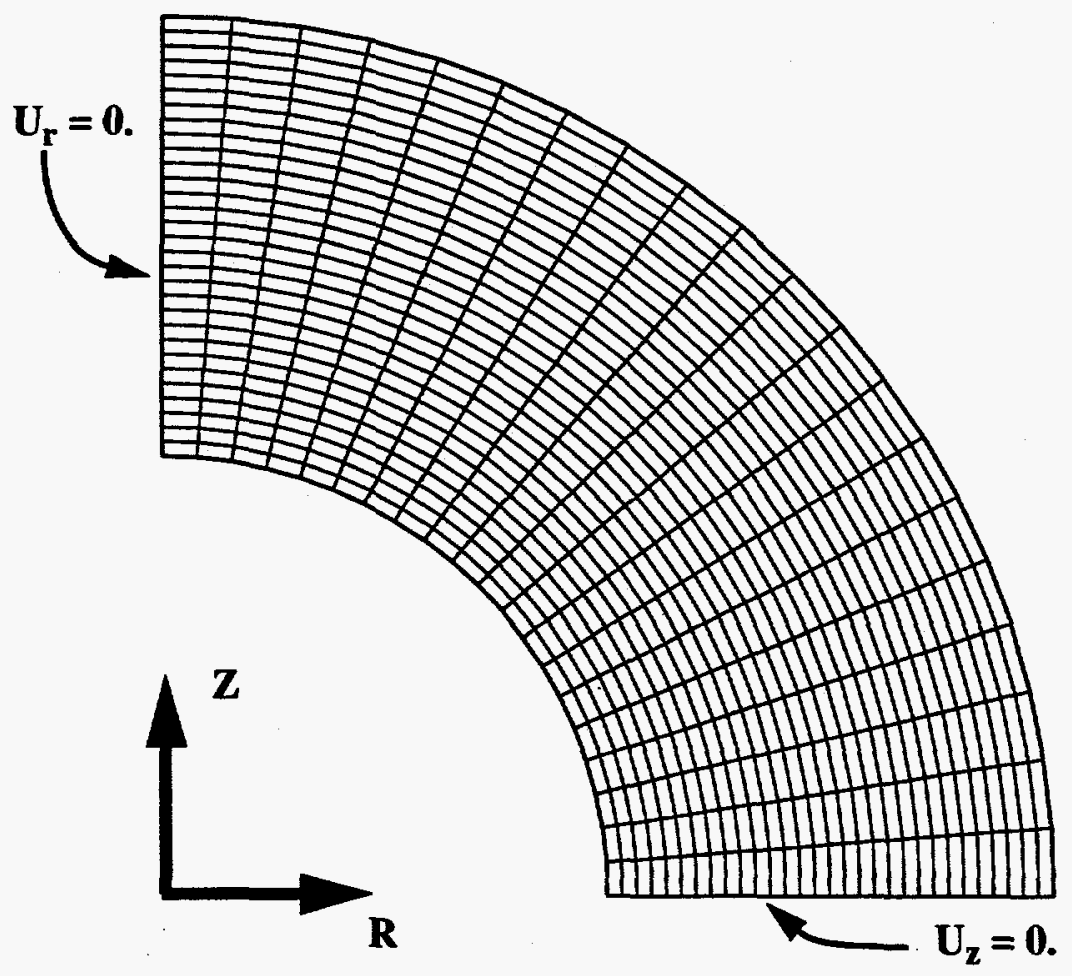

Figure E-8. Finite Element Mesh Discretization Used for the ThickWalled Hollow Sphere Analyses.

In Figures E-9 and E-10, the non-dimensional effective stress is plotted as a function of radius for loadings starting from initial plastic yield at the sphere inner radius to full plastic yielding of the sphere. The analytical solutions are plotted as solid lines in the figures. As can be seen from the plots of normalized effective stress, the computed and analytical results match almost exactly. The only deviation between the solutions is seen in Figure E-9 for the case where the sphere should be fully plastic. The SANTOS solution does not predict a fully plastic sphere. The normalized effective stress for the element at the sphere outer surface does not yield although the pressure applied should induce full plastic yielding of the sphere. It appears that full plastic yielding results in an increase in the calculated outer radius by an amount to stop further yielding and obtain 


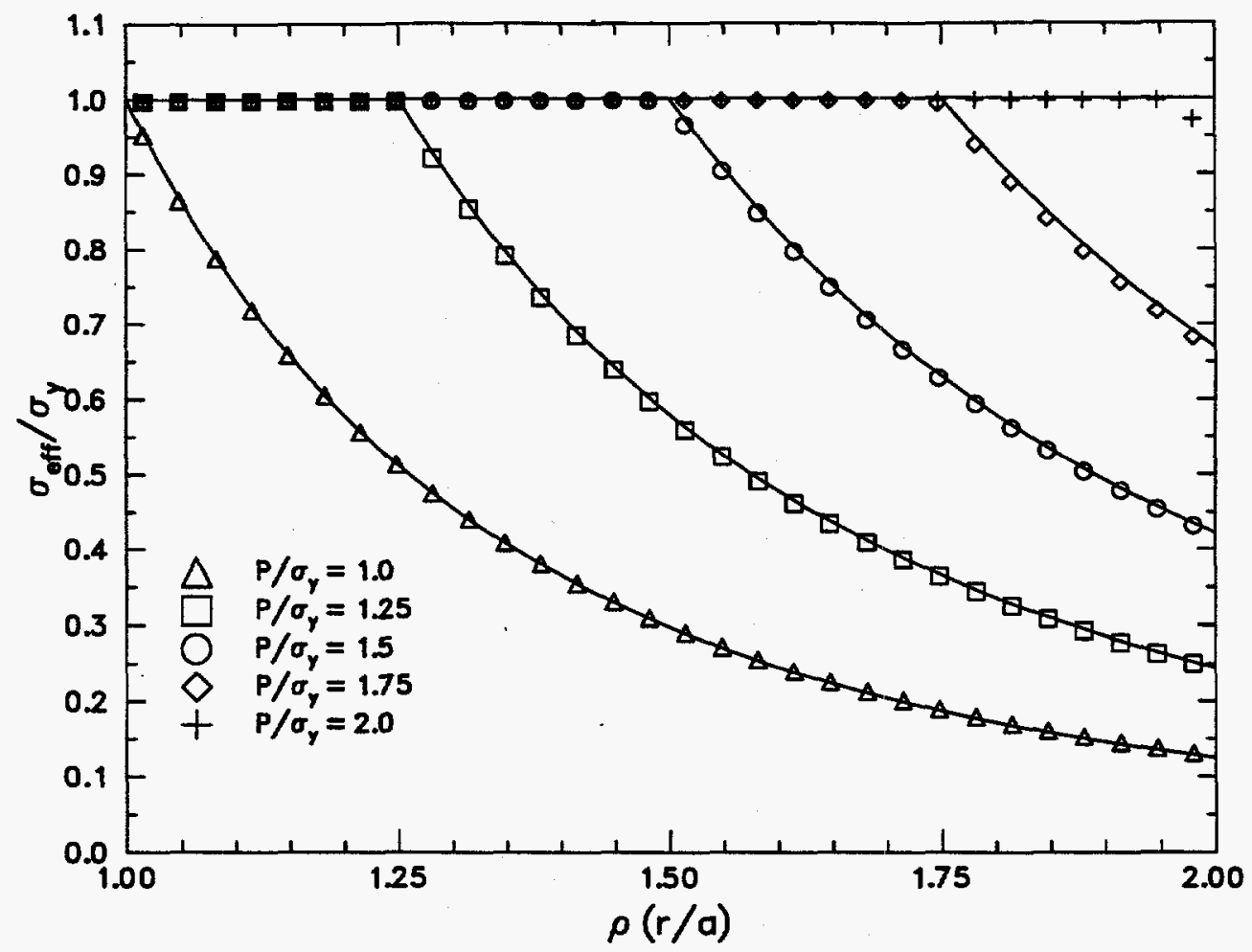

Figure E-9. Normalized Effective Stress Results for the Internally Pressurized Elastic-Perfectly Plastic Thick-Walled Hollow Sphere

equlibrium. In addition, if the sphere were to become fully plastic for an elastic-plastic material with no hardening, the solution would be difficult to converge since the material would be flowing in an unrestrained manner. The SANTOS input file for the internally pressurized elastic-plastic thick-walled hollow sphere is given in Figure E-11.

\section{Upsetting of a Cylindrical Billet}

This verification problem examines the behavior of a cylindrical metallic billet that has undergone a $60 \%$ upset by compression between two flat, rigid dies. The billet has as initial dimensions a length of $30 \mathrm{~mm}$ and a diameter of $20 \mathrm{~mm}$. The axisymmetric option in SANTOS is used and only the top half of the billet is modeled since the middle surface of the billet can be viewed as a plane of symmetry. The time history of the die force is to be compared to computational results by other analysts (Taylor, 1981).

The die material is assumed to be elastic-plastic with linear strain hardening. The material properties are taken from Lippmann (1979). The billet has a Young's modulus of $200 \mathrm{Gpa}$ and a Poisson's ratio of 0.3 . The initial yield stress of the material is $700 \mathrm{Mpa}$ with a hardening modulus of $300 \mathrm{Mpa}$. A uniform mesh containing 216 quadrilateral elements is used. The mesh discretization and boundary conditions used are shown in Figure E-12. The middle surface of the billet is given a prescribed vertical displacement which compresses the billet against the top rigid die. The rigid die is modeled using the RIGID SURFACE option in SANTOS. The die surface is assumed to be rough which results in a no slip condition between the billet and die. This behavior can be achieved by specifying the friction value as FIXED on the RIGID SURFACE option. 


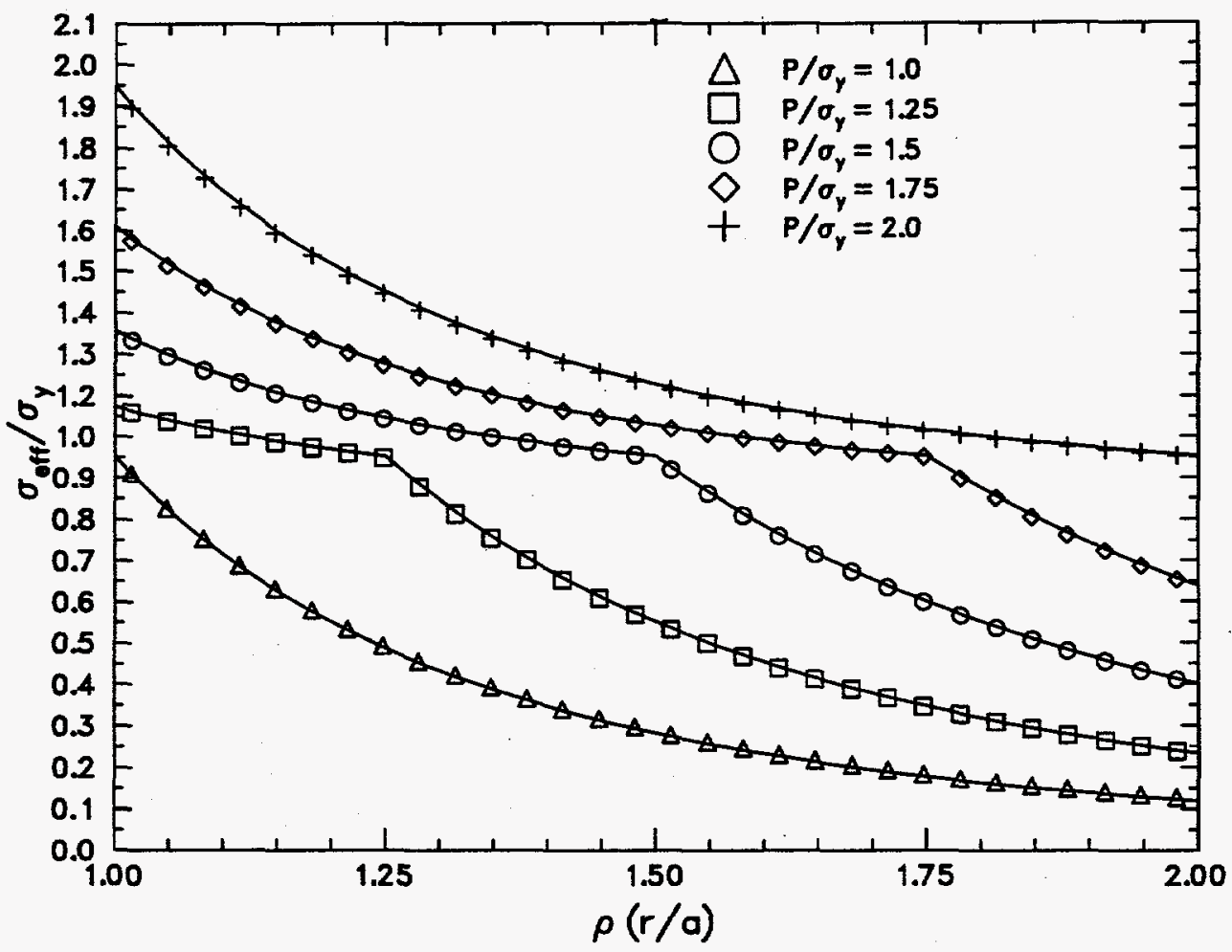

Figure E-10. Normalized Effective Stress Results for the Internally Pressurized Elastic-Plastic Thick-Walled Hollow Sphere With Linear Hardening.

During deformation it is expected that the external surface of the billet will fold and come into contact with the rigid die, which means that the definition of the side set associated with the rigid surface must include both elements along the top of the billet and elements along the external boundary. One hundred load steps were taken for this analysis.

Figure E-13 shows the deformed shape of the billet at several different times during the upset process. The folding of the billet's external surface is clearly seen as well as its contact with the rigid die. A close-up of the billet's final deformed shape at 60\% upset is shown in Figure E-14. Figure E-15 shows a comparison of the upset force vs. die displacement with results taken from Taylor (1981). The agreement is seen to be excellent until the die displacement reaches $7.0 \mathrm{~mm}$. At this value of displacement, the billet is folding and the first nodal point on the external surface is just coming into contact with the rigid surface. The slight difference in the upset force seen in the figure at die displacements greater than $7.0 \mathrm{~mm}$ is related to the contact occurring between the folding billet and the rigid surface. The SANTOS input file for the upsetting of the cylindrical billet is given in Figure E-16.

\section{Closure of a Waste Disposal Room in a Salt Stratigraphy}

Bedded salt is being considered as a storage medium for the long-term disposal of contact-handled transuranic wastes produced as a by-product of the defense activities of the United States. Salt was selected because of its propensity to creep under the action of deviatoric stresses. This creep deformation would eventually entomb the waste and isolate it from the biosphere. Under the 


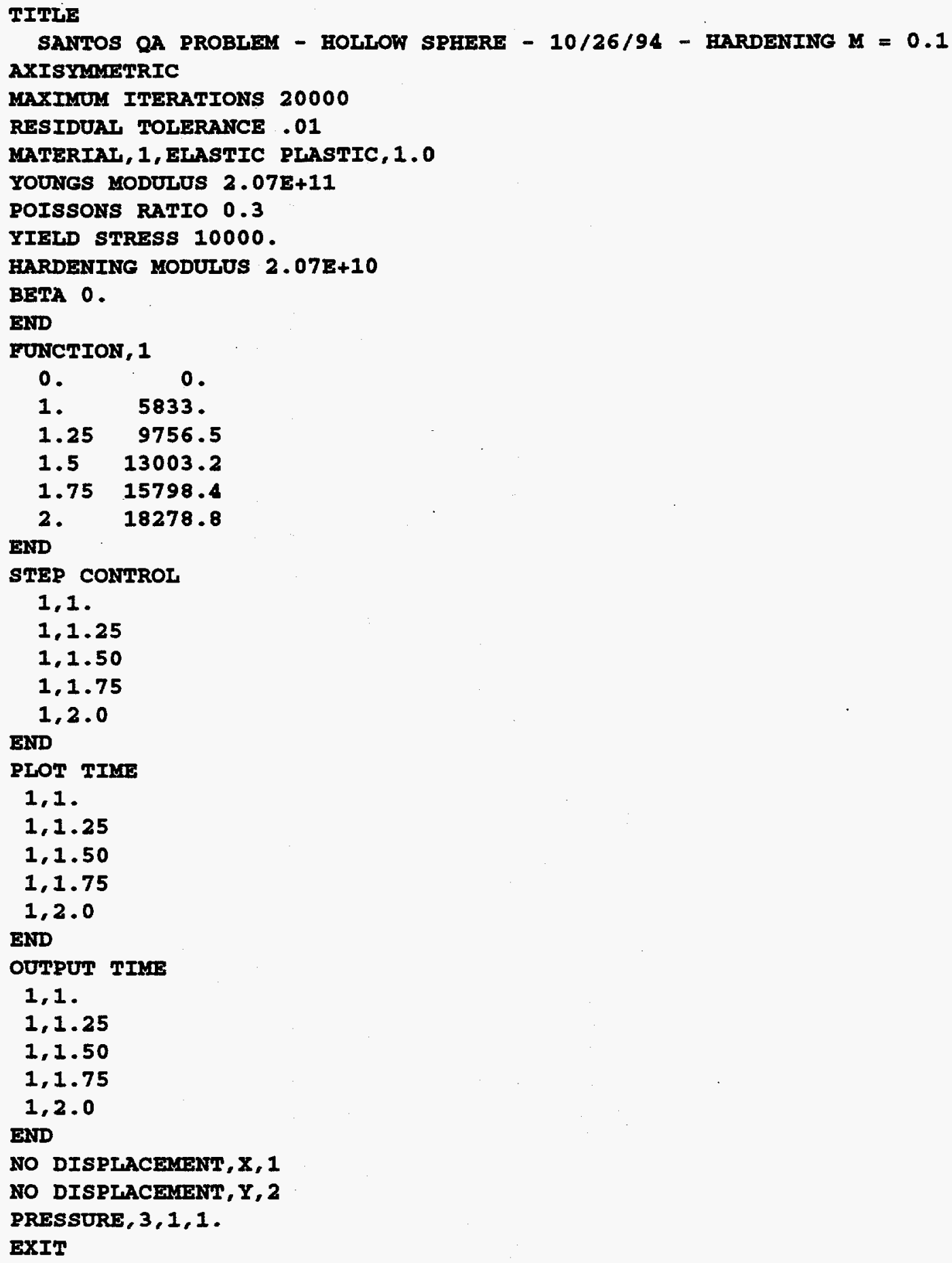

Figure E-11. SANTOS Input File for the Internally Pressurized Elastic-Plastic ThickWalled Hollow Sphere With Linear Hardening.

current plan, the wastes are to be stored in disposal rooms, which are part of a mined respository, $650 \mathrm{~m}$ underground. The disposal rooms are $10.06 \mathrm{~m}$ wide by $3.96 \mathrm{~m}$ high and $91.44 \mathrm{~m}$ in length. As part of the repository performance assessment activity, it was a requirement to determine the 


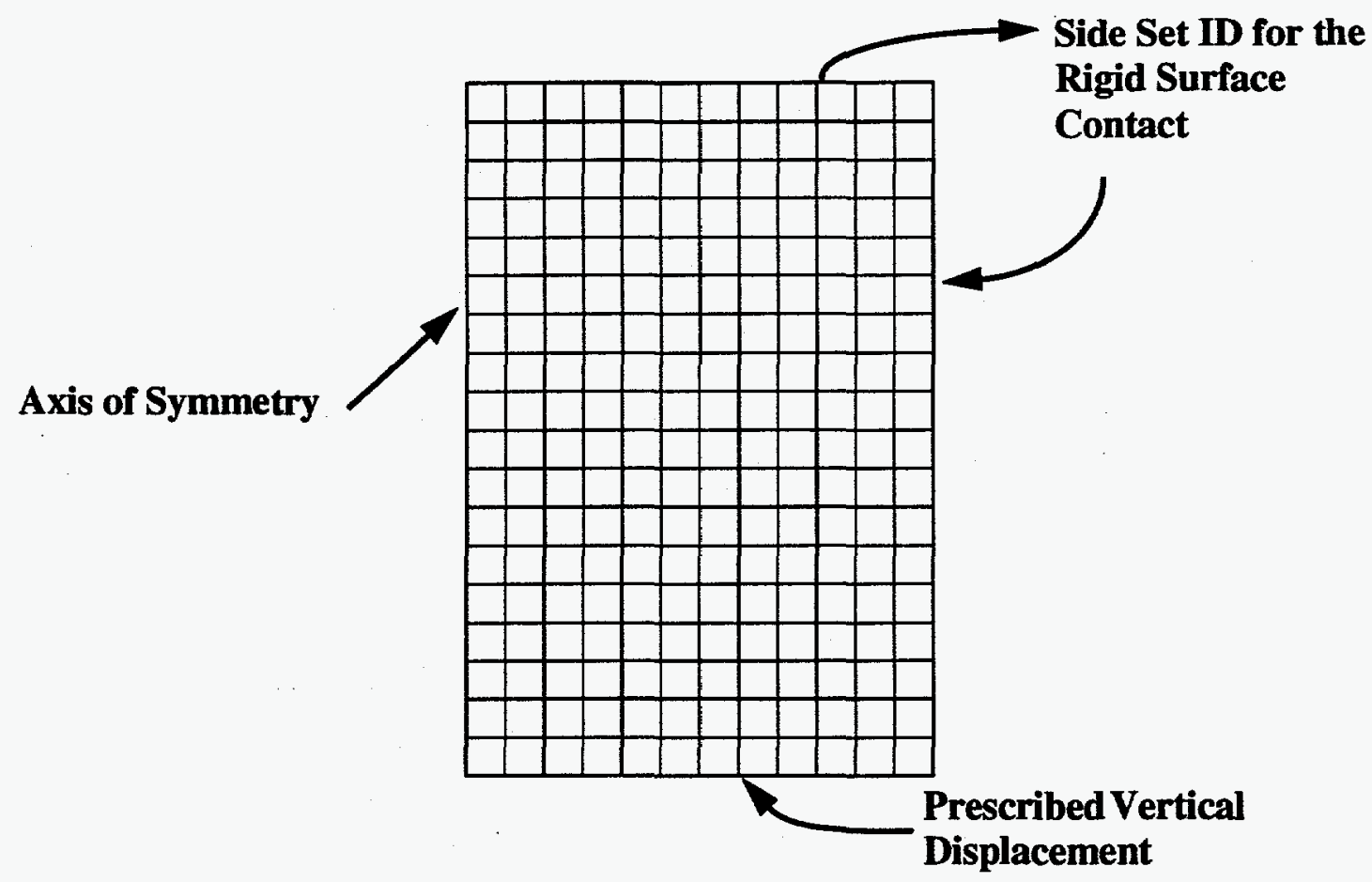

Figure E-12. Mesh Discretization and Boundary Conditions Used for the Analysis of the Upsetting of a Cylindrical Billet.

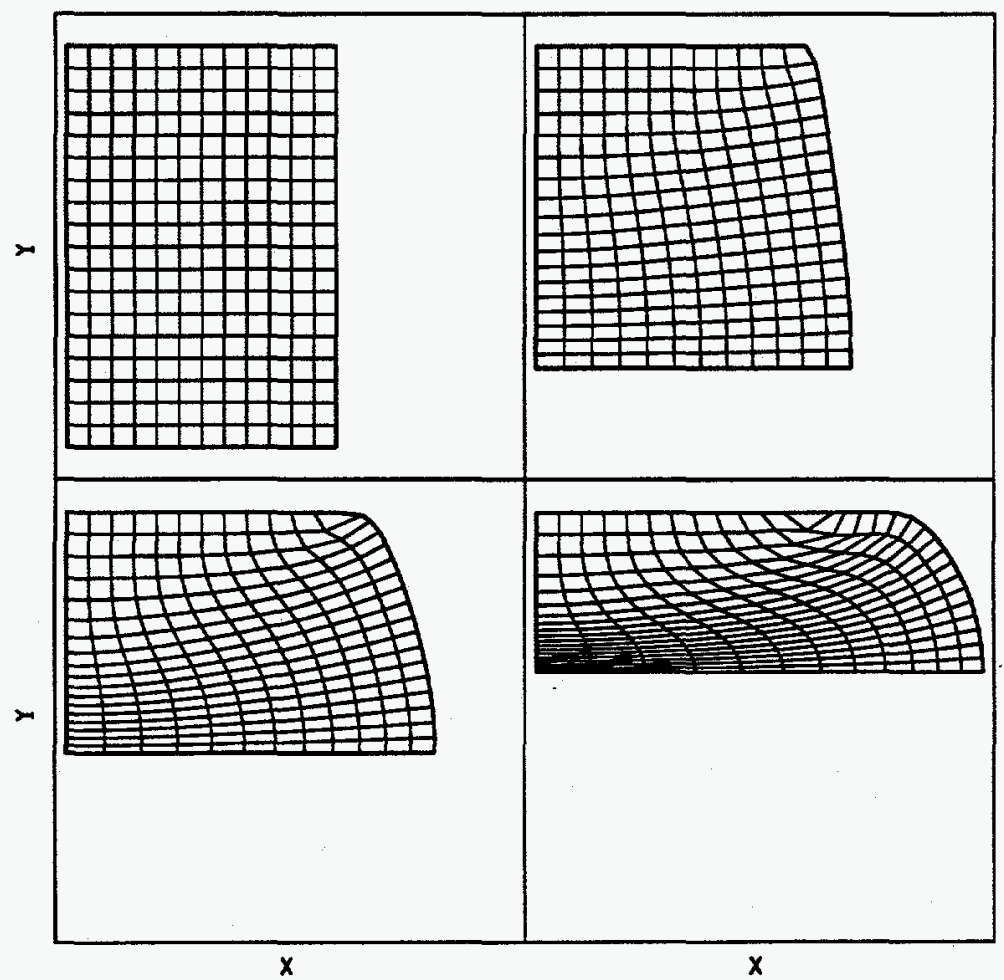

Figure E-13. Plots of the Deforming Billet at Various Times During the Upset. Plots Shown Correspond to Non-Dimensional Times of $0 ., 0.33$, 0.667 , and 1.0 . 


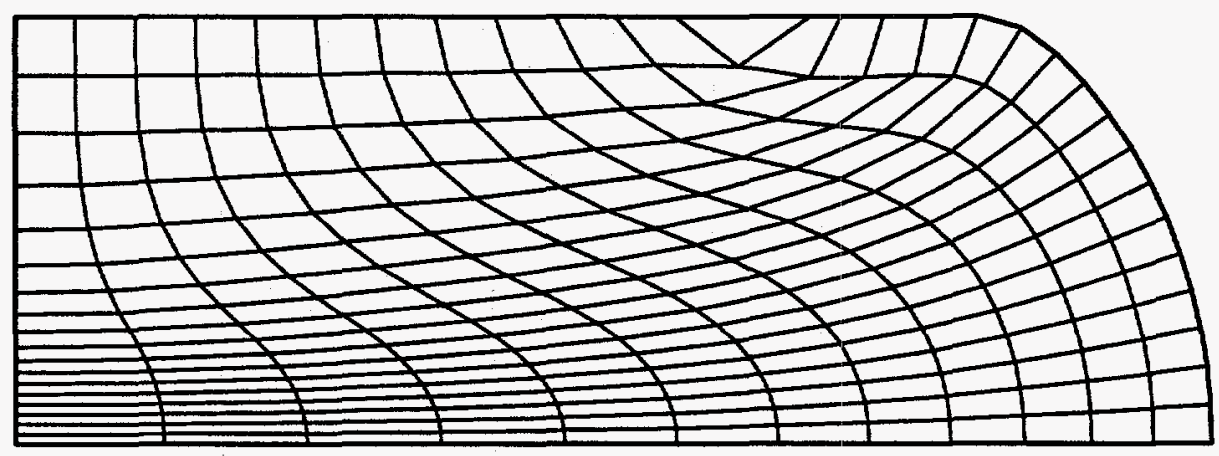

Figure E-14. Final Deformed Shape of the Billet After 60\% Upset.

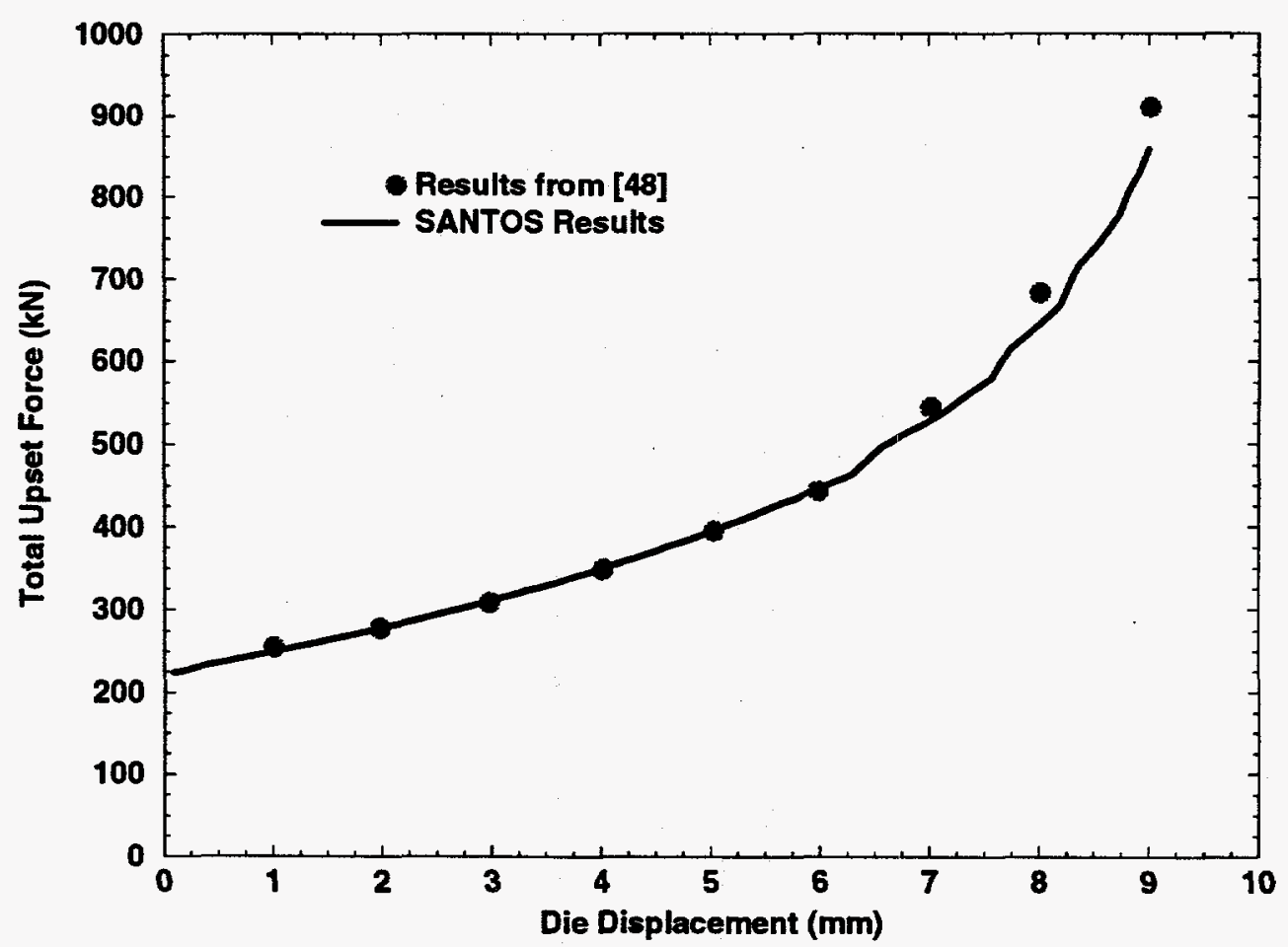

Figure E-15. Comparison of SANTOS Calculation With Numerical Results Taken From Taylor (1981) for the Upset of a Cylindrical Billet.

time required for the disposal room to creep closed. In answering this question, a model of a disposal room in an all salt stratigraphy was developed.

In the disposal room model, it is assumed that the disposal room is one of infinite number of parallel rooms located at the respository horizon. This assumption allows the use of vertical planes of symmetry at the room centerline and in the center of the pillar between rooms which results in the problem geometry shown with the discretized mesh in Figure E-17. The horizontal mesh dimension between symmetry planes is $20.27 \mathrm{~m}$. The vertical mesh boundaries are located 


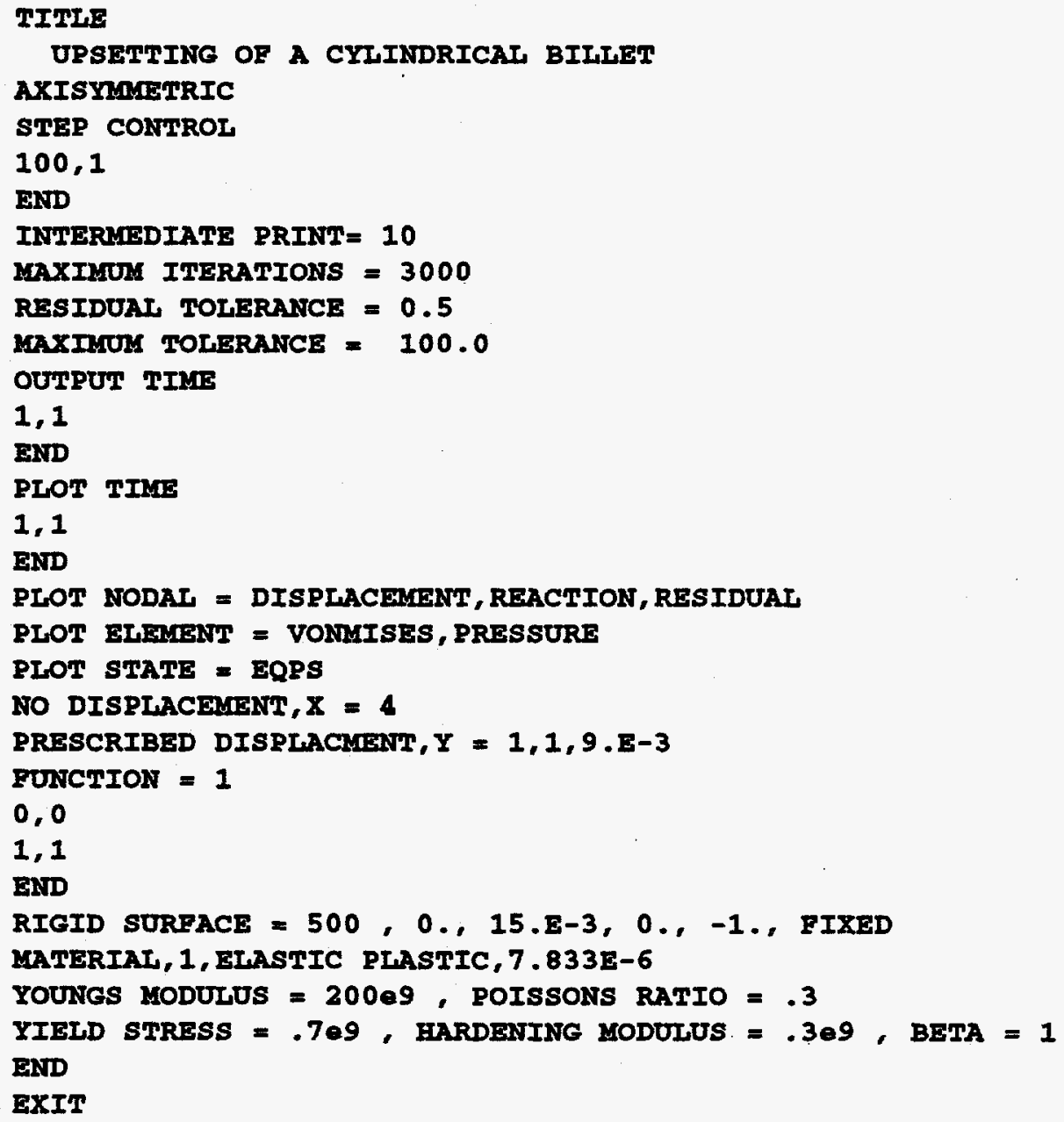

Figure E-16. SANTOS Input File Used for Analyzing the Upsetting of a Cylindrical Billet.

approximately $50 \mathrm{~m}$ from the disposal room. The vertical extent of the problem is designed to remove the effect of the boundaries away from the disposal room. The problem has a traction representing the overburden load applied at the top boundary and the bottom boundary has a traction applied to equilibrate the overburden load plus the additional loads produced by applied gravity forces. An initial hydrostatic stress state is assumed to exist with the value of the stress set to the lithostatic stress. Vertical motion of the model is restrained at a location near the top surface as shown in Figure E-17. Contact surfaces are defined around the interior of the disposal room to accommodate contact that occurs during the large-deformation room closure. Contact pairings are defined between the roof-floor, pillar-roof, and pillar-floor. The coefficient of friction is assumed to be zero for this calculation.

The salt is modeled using the M-D creep model from the SANTOS material library. The M-D model is a combined transient-secondary creep constitutive model for rock salt. The model includes the effects of workhardening and recovery through a state variable function that modifies the steady-state creep rate. The Tresca stress generalization is used in the model for the effective stress definition. The M-D material constants for argillaceous salt are given in Table 1 . The AUTO 


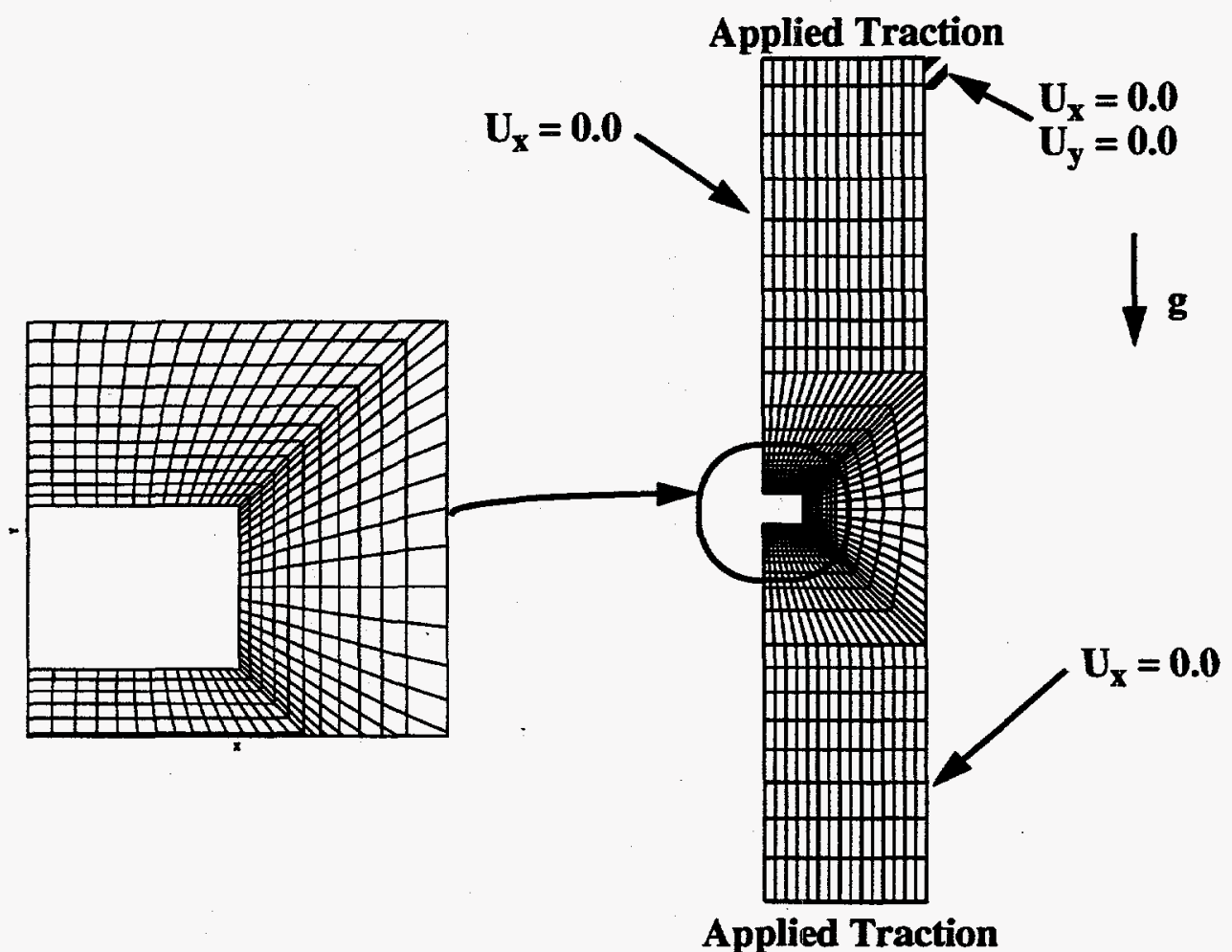

Figure E-17. Geometry, Boundary Conditions and Mesh Discretization for Analyzing the Closure of a Disposal Room in Salt.

STEP option is used with the M-D material model for this problem. The time step necessary for a stable and accurate solution for the M-D model is very small at the start of the analysis. The AUTO STEP option begins with a small initial time step, $1 . \times 10^{-5}$ seconds, and allows it to grow to an analyst specified maximum of $2.592 \times 10^{6}$ seconds. Without invoking the AUTO STEP option, it is very time consuming to perform this analysis.

Figure E-18 shows the deformed shape of the disposal room at several different times from the initial undeformed state to final closure. The closure process is characterized by shortening of the pillar and an inward displacement of the disposal room roof and floor. Contact between the roofpillar and floor-pillar occurs near the room corners. As contact occurs, the rate of room closure slows as the pillar begins to support the roof and floor. A close-up of the disposal room at closure is shown in Figure E-19. The contact of the disposal room interior surfaces is clearly shown in this figure. Figure E-20 shows the time history of room closure as measured by the sum of the displacements of the floor and roof centerline nodal points. When the sum of the displacements reaches $3.96 \mathrm{~m}$ then the floor and roof have come into contact and the disposal room is assumed to be closed. Closure is seen to occur at approximately 57 years. The SANTOS input file for analyzing the closure of a waste disposal room in salt is given in Figure E-21. 
Table 1: M-D Argillaceous Salt Creep Properties

\begin{tabular}{|l|c|}
\hline \multicolumn{1}{|c|}{$\begin{array}{c}\text { Parameters } \\
\text { (units) }\end{array}$} & $\begin{array}{c}\text { Parameter } \\
\text { Value }\end{array}$ \\
\hline \hline$G(\mathrm{MPa})$ & 12,400 \\
\hline$E(\mathrm{MPa})$ & 31,000 \\
\hline$v$ & 0.25 \\
\hline$A_{1}(/ \mathrm{sec})$ & $1.407 \mathrm{E} 23$ \\
\hline$Q_{1}(\mathrm{cal} / \mathrm{mole})$ & 25,000 \\
\hline$n_{1}$ & 5.5 \\
\hline$B_{1}(/ \mathrm{sec})$ & $8.998 \mathrm{E} 6$ \\
\hline$A_{2}(/ \mathrm{sec})$ & $1.314 \mathrm{E} 13$ \\
\hline$Q_{2}(\mathrm{cal} / \mathrm{mole})$ & 10,000 \\
\hline$n_{2}$ & 5.0 \\
\hline$B_{2}(/ \mathrm{sec})$ & $4.289 \mathrm{E}-2$ \\
\hline$\sigma_{o}(\mathrm{MPa})$ & 20.57 \\
\hline$q$ & 5,335 \\
\hline$M$ & 3.0 \\
\hline$K_{o}$ & $2.470 \mathrm{E} 6$ \\
\hline$c(/ \mathrm{T})$ & $9.198 \mathrm{E}-3$ \\
\hline$\alpha$ & -14.96 \\
\hline$\beta$ & -7.738 \\
\hline$\delta$ & 0.58 \\
\hline
\end{tabular}




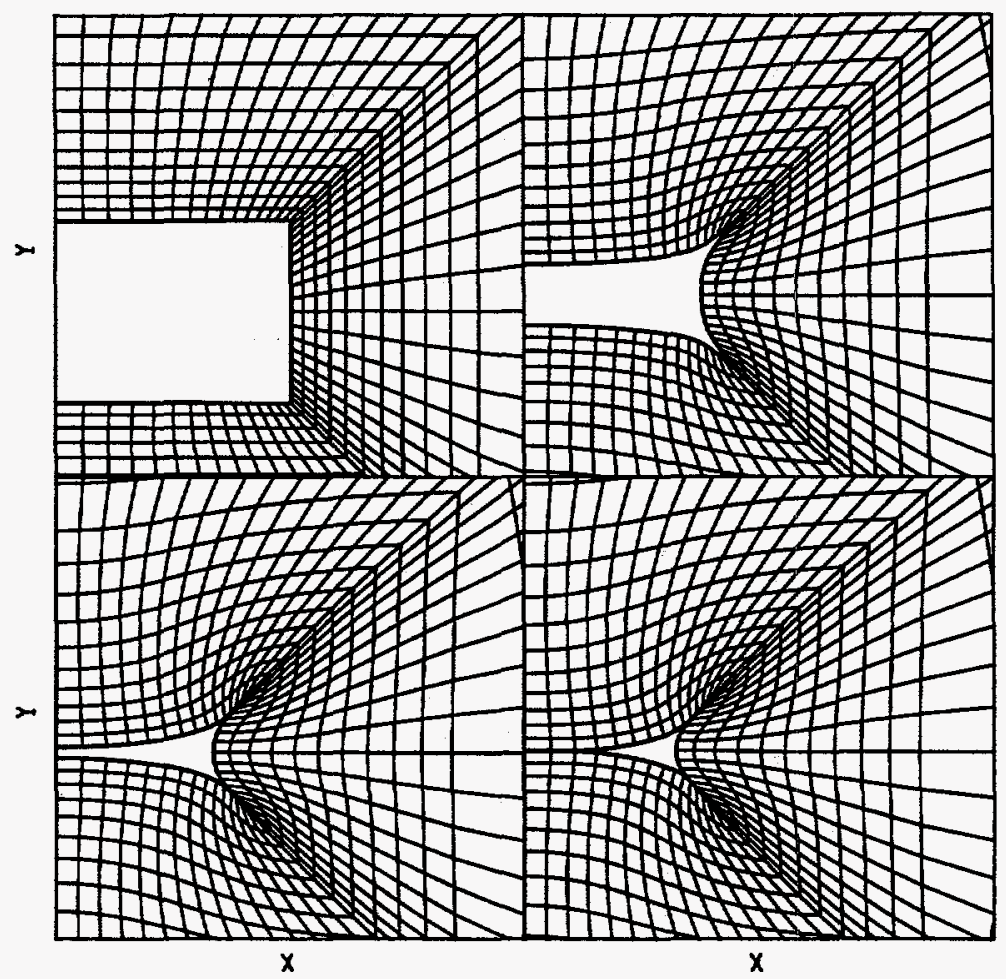

Figure E-18. Plots of the Deforming Disposal Room at Selected Times From Initial Excavation to Final Closure. Times 0., 25. 50., and 80 Years.

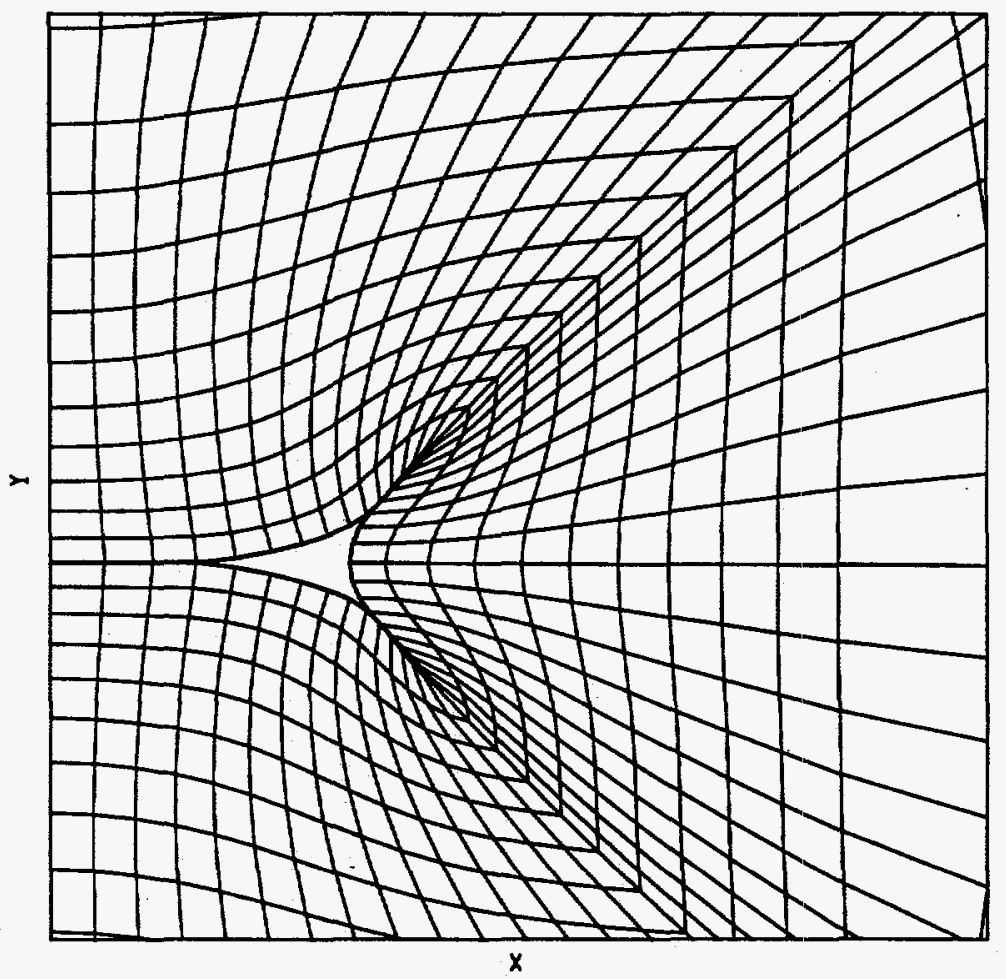

Figure E-19. Deformed Shape of the Disposal Room 100 Years After Excavation. 


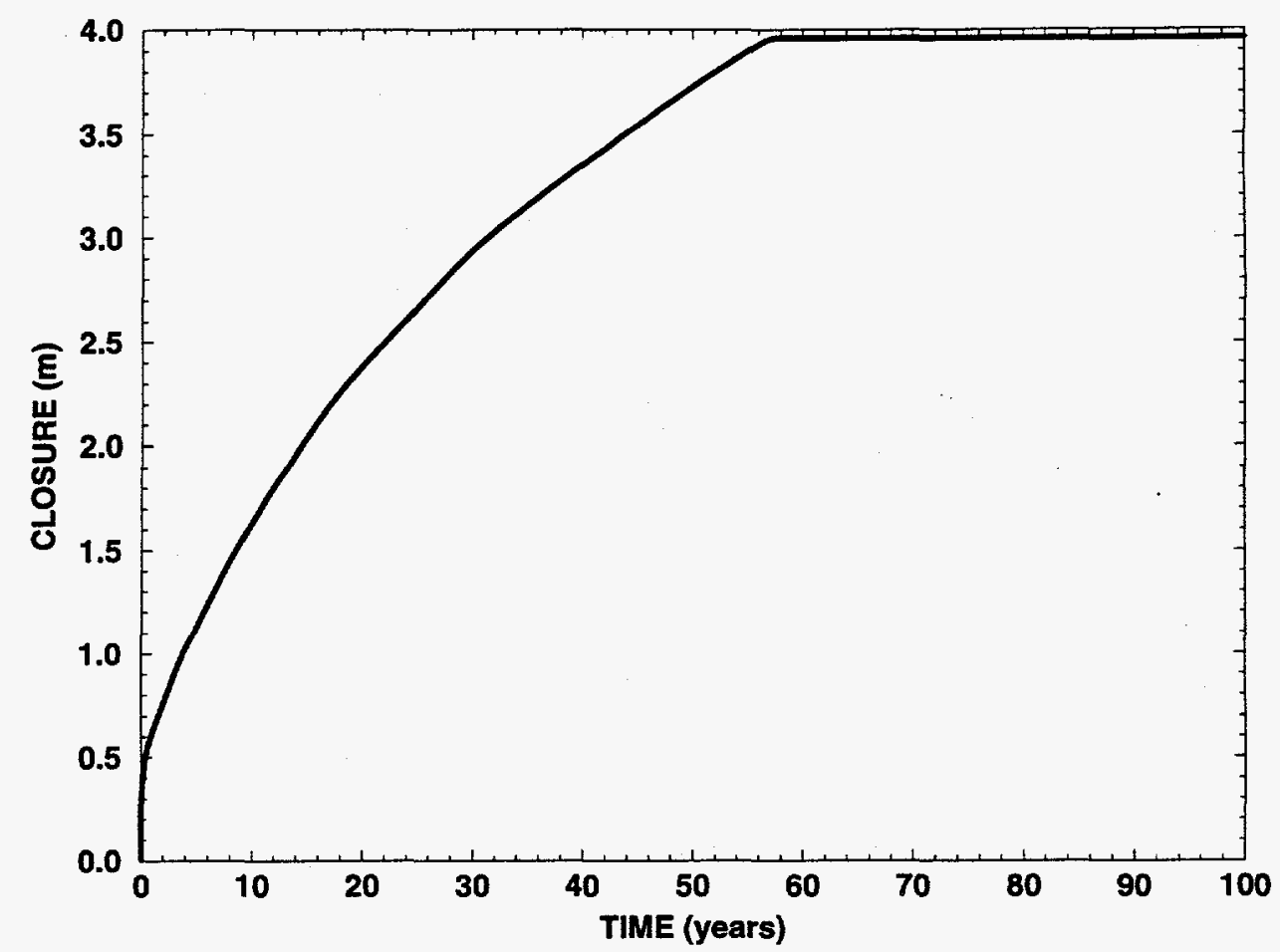

Figure E-20. Closure History of the Disposal Room Centerline. Contact of the Floor and Roof is Reached at Approximately 57 Years.

\section{References}

Holden, J. T. 1972. "On the Finite Deflections of Thin Beams," International Journal of Solids and Structures. Vol. 8, 1051-1055.

Lippmann, H., ed. 1979. Metal Forming Plasticity. New York, NY: Springer-Verlag.

Mendelson, A. 1968. Plasticity: Theory and Application. New York, NY: The Macmillan Company. 138-156.

Taylor, L.M. 1981. "A Finite Element Analysis for Large Deformation Metal Forming Problems Involving Contact and Friction." Ph.D. dissertation. Austin, TX: University of Texas at Austin. 


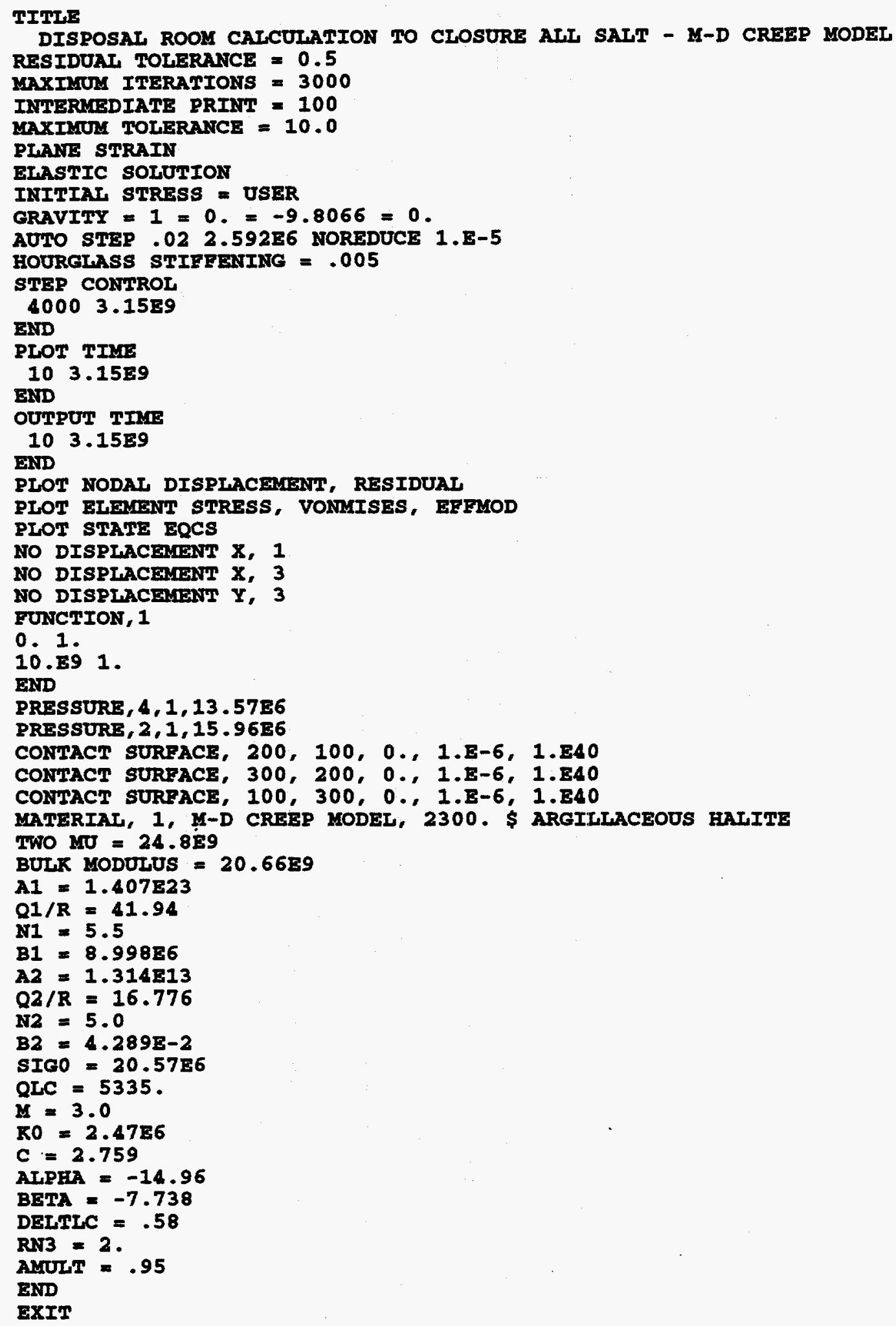

Figure E-21. SANTOS Input File for Analyzing the Closure of a Waste Disposal Room in Salt. 


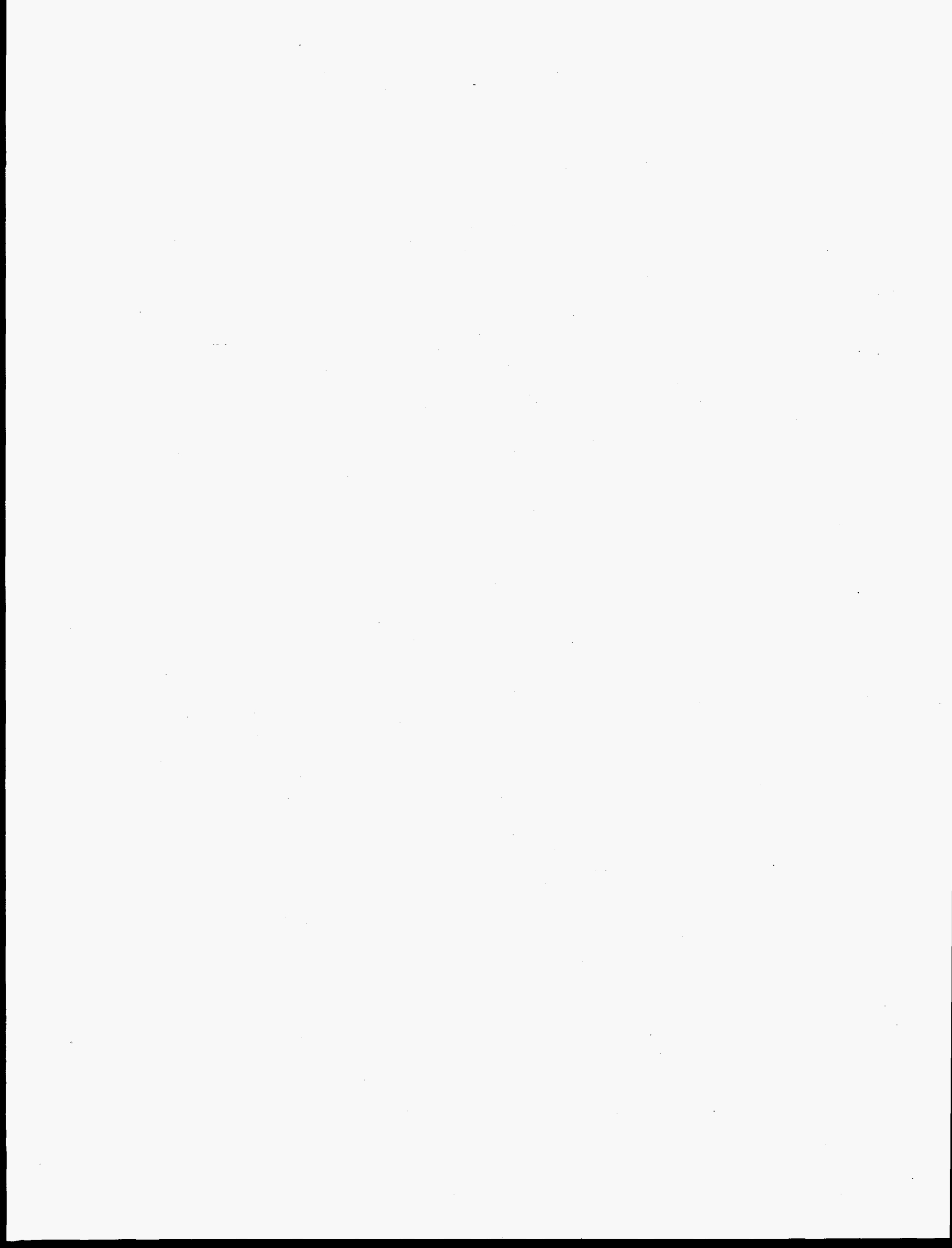





\section{WIPP \\ UC721 - DISTRIBUTION LIST \\ SAND90-0543}

\section{Federal Agencies}

US Department of Energy (4)

Office of Civilian Radioactive Waste Mgmt.

Attn: Deputy Director, RW-2

Acting Director, RW-10

Office of Human Resources \& Admin.

Director, RW-30

Office of Program Mgmt and Admin.

Associate Director, RW-40

Office of Waste Accept., Stor., \& Tran.

Forrestal Building

Washington, DC 20585

US Department of Energy

Albuquerque Operations Office

Attn: National Atomic Museum Library

P.O. Box 5400

Albuquerque, NM 87185-5400

US Department of Energy

Research \& Waste Management Division

Attn: Director

P.O. Box E

Oak Ridge, TN 37831

US Department of Energy (5)

Carlsbad Area Office

Attn: G. Dials

D. Galbraith

M. McFadden

R. Lark

J. A. Mewhinney

P.O. Box 3090

Carlsbad, NM 88221-3090

US Department of Energy

Office of Environmental Restoration and

Waste Management

Attn: M. Frei, EM-30

Forrestal Building

Washington, DC 20585-0002

US Department of Energy (3)

Office of Environmental Restoration and

Waste Management

Attn: J. Juri, EM-34, Trevion II

Washington, DC 20585-0002
US Department of Energy

Office of Environmental Restoration and Waste Management

Attn: S. Schneider, EM-342, Trevion II

Washington, DC 20585-0002

US Department of Energy (2)

Office of Environment, Safety \& Health

Attn: C. Borgstrom, EH-25

R. Pelletier, EH-231

Washington, DC 20585

US Department of Energy (2)

Idaho Operations Office

Fuel Processing \& Waste Mgmt. Division

785 DOE Place

Idaho Falls, ID 83402

US Environmental Protection Agency (2)

Radiation Protection Programs

Attn: M. Oge

ANR-460

Washington, DC 20460

Attn: Project Director

Yucca Mountain Site Characterization Office Director, RW-3

Office of Quality Assurance

P. O. Box 30307

Las Vegas, NV 89036-0307

Boards

Defense Nuclear Facilities Safety Board

Attn: D. Winters

625 Indiana Ave. NW, Suite 700

Washington, DC 20004

Nuclear Waste Technical Review Board (2)

Attn: Chairman

J. L. Cohon

1100 Wilson Blvd., Suite 910

Arlington, VA 22209-2297

\section{State Agencies}

Attorney General of New Mexico

P.O. Drawer 1508

Santa Fe, NM 87504-1508 
Environmental Evaluation Group (3)

Attn: Library

7007 Wyoming NE

Suite F-2

Albuquerque, NM 87109

NM Energy, Minerals, and Natural

Resources Department

Attn: Library

2040 S. Pacheco

Santa Fe, NM 87505

NM Environment Department (3)

Secretary of the Environment

Attn: Mark Weidler

1190 St. Francis Drive

Santa Fe, NM 87503-0968

NM Bureau of Mines \& Mineral Resources Socorro, NM 87801

\section{Laboratories/Corporations}

Battelle Pacific Northwest Laboratories Battelle Blvd.

Richland, WA 99352

Los Alamos National Laboratory

Attn: B. Erdal, INC-12

P.O. Box 1663

Los Alamos, NM 87544

Tech Reps, Inc. (3)

Attn: J. Chapman (1)

Loretta Robledo (2)

5000 Marble NE, Suite 222

Albuquerque, NM 87110

Westinghouse Electric Corporation (5)

Attn: Library

J. Epstein

J. Lee

B. A. Howard

R. Kehrman

P.O. Box 2078

Carlsbad, NM 88221

S. Cohen \& Associates

Attn: Bill Thurber

1355 Beverly Road

McLean, VA 22101

\section{National Academy of Sciences,} WIPP Panel

Howard Adler

Oxyrase, Incorporated

7327 Oak Ridge Highway

Knoxville, TN 37931

Tom Kiess

Board of Radioactive Waste Management

GF456

2101 Constitution Ave.

Washington, DC 20418

Rodney C. Ewing

Department of Geology

University of New Mexico

Albuquerque, NM 87131

Charles Fairhurst

Department of Civil and Mineral Engineering

University of Minnesota

500 Pillsbury Dr. SE

Minneapolis, MN 55455-0220

B. John Garrick

PLG Incorporated

4590 MacArthur Blvd., Suite 400

Newport Beach, CA 92660-2027

Leonard F. Konikow

US Geological Survey

431 National Center

Reston, VA 22092

Carl A. Anderson, Director

Board of Radioactive Waste Management

National Research Council

HA 456

2101 Constitution Ave. NW

Washington, DC 20418

Christopher G. Whipple

ICF Kaiser Engineers

1800 Harrison St., 7th Floor

Oakland, CA. 94612-3430

John O. Blomeke

720 Clubhouse Way

Knoxville, TN 37909 
Sue B. Clark

University of Georgia

Savannah River Ecology Lab

P.O. Drawer E

Aiken, SC 29802

Konrad B. Krauskopf

Department of Geology

Stanford University

Stanford, CA 94305-2115

Della Roy

Pennsylvania State University

217 Materials Research Lab

Hastings Road

University Park, PA 16802

David A. Waite

$\mathrm{CH}_{2} \mathrm{M}$ Hill

P.O. Box 91500

Bellevue, WA 98009-2050

Thomas A. Zordon

Zordan Associates, Inc.

3807 Edinburg Drive

Murrysville, PA 15668

\section{Universities}

University of New Mexico

Geology Department

Attn: Library

141 Northrop Hall

Albuquerque, NM 87131

University of Washington

College of Ocean \& Fishery Sciences

Attn: G. R. Heath

583 Henderson Hall, HN-15

Seattle, WA 98195

\section{Libraries}

Thomas Brannigan Library

Attn: D. Dresp

$106 \mathrm{~W}$. Hadley St.

Las Cruces, NM 88001

Government Publications Department

Zimmerman Library

University of New Mexico

Albuquerque, NM 87131
New Mexico Junior College

Pannell Library

Attn: R. Hill

Lovington Highway

Hobbs, NM 88240

New Mexico State Library

Attn: N. McCallan

325 Don Gaspar

Santa Fe, NM 87503

New Mexico Tech

Martin Speere Memorial Library

Campus Street

Socorro, NM 87810

WIPP Public Reading Room

Carlsbad Public Library

101 S. Halagueno St.

Carlsbad, NM 88220

\section{Foreign Addresses}

Atomic Energy of Canada, Ltd.

Whiteshell Laboratories

Attn: B. Goodwin

Pinawa, Manitoba, CANADA R0E ILO

Francois Chenevier (2)

ANDRA

Route de Panorama Robert Schumann

B. P. 38

92266 Fontenay-aux-Roses, Cedex

FRANCE

Claude Sombret

Centre d'Etudes Nucleaires de la Vallee Rhone

CEN/VALRHO

S.D.H.A. B.P. 171

30205 Bagnols-Sur-Ceze, FRANCE

Commissariat a L'Energie Atomique

Attn: D. Alexandre

Centre d'Etudes de Cadarache

13108 Saint Paul Lez Durance Cedex

FRANCE

Bundesanstalt fur Geowissenschaften und

Rohstoffe

Attn: M. Langer

Postfach 510153

D-30631 Hannover, GERMANY . 
Bundesministerium fur Forschung und

Technologie

Postfach 200706

5300 Bonn 2, GERMANY

Institut fur Tieflagerung

Attn: K. Kuhn

Theodor-Heuss-Strasse 4

D-3300 Braunschweig, GERMANY

Gesellschaft fur Anlagen und Reaktorsicherheit (GRS)

Attn: B. Baltes

Schwertnergasse 1

D-50667 Cologne, GERMANY

\section{Shingo Tashiro}

Japan Atomic Energy Research Institute

Tokai-Mura, Ibaraki-Ken, 319-11

JAPAN

Netherlands Energy Research Foundation ECN

Attn: J. Prij

3 Westerduinweg

P.O. Box 1

1755 ZG Petten

THE NETHERLANDS

Svensk Karnbransleforsorjning $A B$

Attn: F. Karlsson

Project KBS (Karnbranslesakerhet)

Box 5864

S-102 48 Stockholm

SWEDEN

Nationale Genossenschaft fur die Lagerung

Radioaktiver Abfalle (2)

Attn: S. Vomvoris

P. Zuidema

Hardstrasse 73

CH-5430 Wettingen

SWITZERLAND

AEA Technology

Attn: J. H. Rees

D5W/29 Culham Laboratory

Abington, Oxfordshire OX14 3DB

UNITED KINGDOM

AEA Technology

Attn: W. R. Rodwell

044/A31 Winfrith Technical Centre

Dorchester, Dorset DT2 8DH

UNITED KINGDOM
AEA Technology

Attn: J. E. Tinson

B4244 Harwell Laboratory

Didcot, Oxfordshire OXIl ORA

UNITED KINGDOM

\section{Additional External Recipients}

C. A. Anderson

MEE-13, MS J576

Los Alamos National Laboratory

Los Alamos, NM 87545

Prof. S. Atluri

Center for the Advancement of

Computational Mechanics

School of Civil Engineering

Georgia Institute of Technology

Atlanta, GA 30332

Prof. E. B. Becker

Department of Aerospace Engineering and Engineering Mechanics

University of Texas/Austin

Austin, TX 78712-1085

Prof. T. Belytschko

Department of Civil Engineering

Northwestern University

Evanston, IL 60201

Prof. Dave Benson

Department of Applied Mechanics and Engineering Sciences

UC San Diego

La Jolla, CA 92093

Dr. Gary Callahan

RE/SPEC Inc.

Box 725

Rapid City, SD 57709

Dr. Tom Canfield

Argonne National Laboratory

9700 S. Cass Ave. CTD/221

Argonne, II 60439-4844

W. A. Cook

N-6, MS K557

Los Alamos National Laboratory

Los Alamos, NM 87545 
Mr. Steven Crouch

GeoLogic Research, Inc.

1313 Fifth St. SE, Suite 226

Minneapolis, MN 55414

Mr. Peter Cundall

ITASCA Consulting Group, Inc.

1313 Fifth Street, SE

Minneapolis, MN 55414

Dr. R. A. Dameron

Anatech International Corp.

5435 Oberlin Drive

San Diego, CA 92121

Dr. R. S. Dunham

Anatech International Corp.

5435 Oberlin Drive

San Diego, CA 92121

Dr. D. P. Flanagan

Hibbitt, Karlsson \& Sorresen, Inc.

100 Medway St.

Providence, RI 02906

Dr. Gerry Goudreau

Methods Development Group

Mechanical Engineering Department

Lawrence Livermore National Laboratory

Livermore, CA 94550

F. Guerra

WX-11, MS C931

Los Alamos National Laboratory

Los Alamos, NM 87545

Dr. David Hibbitt

Hibbitt, Karlsson \& Sorrensen, Inc.

100 Medway Street

Providence, RI 02906

J. P. Hill

WX-11, MS C931

Los Alamos National Laboratory

Los Alamos, NM 87545

Prof. T. J. R. Hughes

Dept. of Mechanical Engineering

Stanford University

Palo Alto, CA 94306
Prof. Raymond D. Krieg

Engineering Science and Mechanics

301 Perkins Hall

University of Tennessee

Knoxville, TN 37996-2030

Dr. Duane Labreche

RE/SPEC Inc.

4775 Indian School Road NE

Suite 300

Albuquerque, NM 871 10-3827

Mr. Loren K. Miller

Goodyear Technical Center

P. O. Box 3531

Akron, OH 44309-3531

Dr. R. E. Nickell

c/o Anatech International Corp.

5435 Oberlin Drive

San Diego, CA 92121

Prof. J. T. Oden

Department of Aerospace Engineering and Engineering Mechanics

University of Texas/Austin

Austin, TX 78712-1085

Dr. Joe Rashid

Anatech International Corp.

5435 Oberlin Drive

San Diego, CA 92121

Prof. Mark Rashid

Department of Civil \& Environmental Eng.

University of California

Davis, CA 95616-5294

J. J. Ruminer

WX-11, MS C931

Los Alamos National Laboratory

Los Alamos, NM 87545

Prof. M. Stern

Department of Aerospace Engineering and Engineering Mechanics

University of Texas/Austin

Austin, TX 78712-1085

Prof. D. V. Swenson

Mechanical Engineering Department

Kansas State University

Manhattan, KS 66506 
Dr. Krishan K. Wahi

Gram, Inc.

1709 Moon NE

Albuquerque, NM 87112

Prof. John Wilson

Department of Geoscience

NM Institute of Mining \& Technology

Socorro, NM 87801

\section{Internal}

\begin{tabular}{|c|c|c|}
\hline MS & Org. & \\
\hline 1324 & 6115 & P. B. Davies \\
\hline 1320 & 6831 & E. J. Nowak \\
\hline 1322 & 6121 & J. R. Tillerson \\
\hline 1328 & 6849 & D. R. Anderson \\
\hline 1328 & 6848 & H. N. Jow \\
\hline 1335 & 6801 & M. Chu \\
\hline 1341 & 6832 & J. T. Holmes \\
\hline 1395 & 6800 & L. Shephard \\
\hline 1395 & 6821 & M. Marietta \\
\hline 1395 & 6841 & V. H. Slaboszewicz \\
\hline 0439 & 9234 & D. Martinez \\
\hline 0819 & 9231 & M. McGlaun \\
\hline 0820 & 9232 & P. Yarrington \\
\hline 0833 & 9103 & J. H. Biffle \\
\hline 0828 & 9104 & E. D. Gorham \\
\hline 0827 & 9114 & R. T. McGrath \\
\hline 0827 & 9111 & D. K. Gartling \\
\hline 0834 & 9112 & A. C. Ratzel \\
\hline 0834 & 9112 & R. J. Gross \\
\hline 0834 & 9112 & M. L. Hobbs \\
\hline 0835 & 9102 & R. D. Skocypec \\
\hline 0826 & 9111 & W. Hermina \\
\hline 0825 & 9115 & W. H. Rutledge \\
\hline 0836 & 9116 & C. W. Peterson \\
\hline 0443 & 9117 & H. S. Morgan and Staff (21) \\
\hline 0443 & 9117 & C. M. Stone (30) \\
\hline 0437 & 9118 & R. K. Thomas \& Staff (16) \\
\hline 0751 & 6117 & D. S. Preece \\
\hline 0706 & 6113 & D. E. Munson \\
\hline 9043 & 8742 & E. P. Chen: Route to Staff \\
\hline 9405 & 8743 & P. Nielan: Route to Staff \\
\hline 1330 & 6811 & K. Hart (2) \\
\hline 1330 & 4415 & NWM Library (20) \\
\hline 9018 & 8940-2 & Central Technical Files \\
\hline 0899 & 4414 & Technical Library (5) \\
\hline 0619 & 12690 & $\begin{array}{l}\text { Review and Approval Desk (2) } \\
\text { for DOE/OSTI }\end{array}$ \\
\hline
\end{tabular}

